Hand-finger pose tracking using

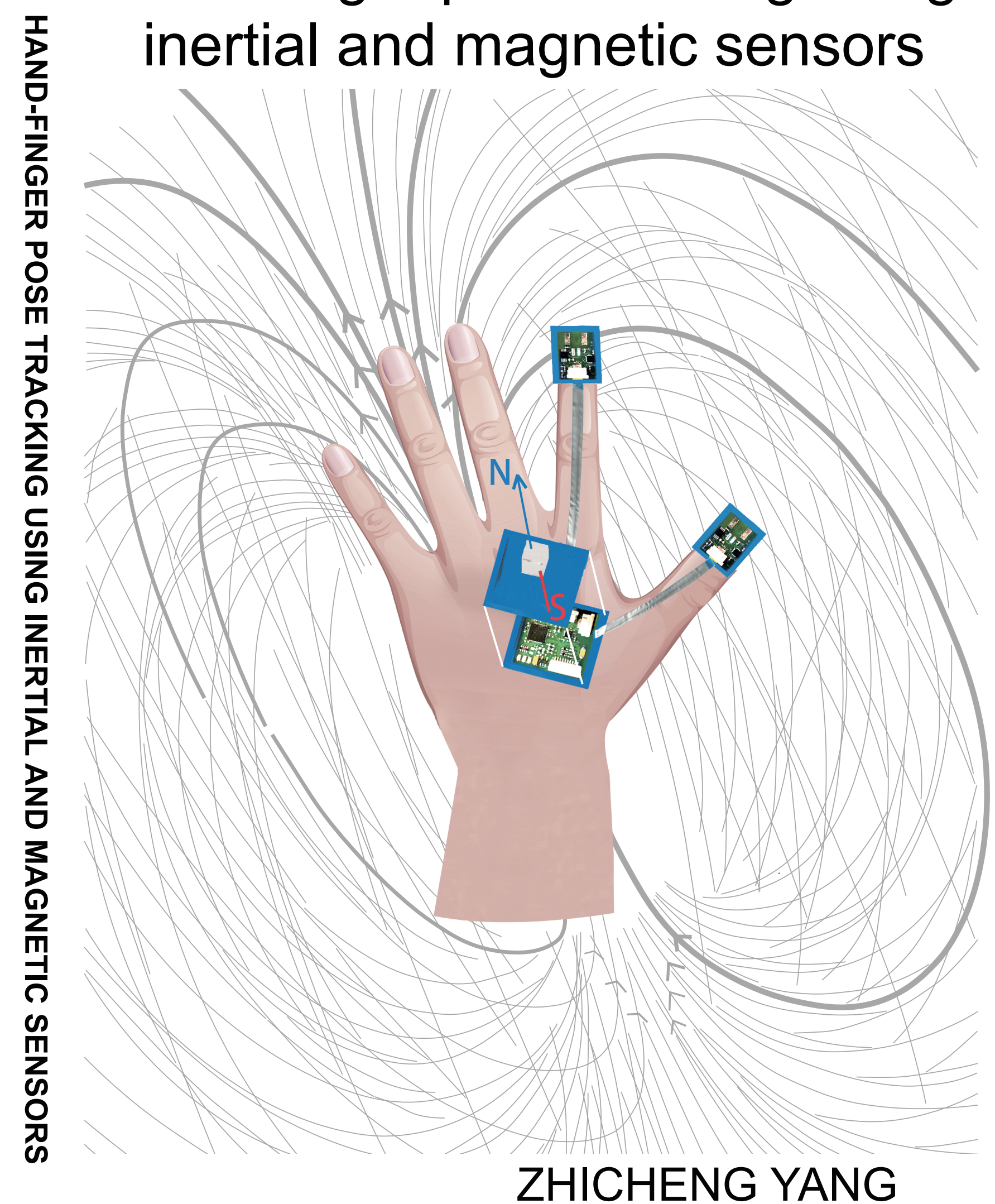

\section{Invitation}

to the public defense of my PhD dissertation

HAND-FINGER POSE TRACKING USING INERTIAL AND MAGNETIC SENSORS

Thursday

24 February 2022

at $14: 45$

University of Twente

杨志成

Zhicheng Yang zcyang007@126.com 


\section{Hand-finger pose tracking using inertial and magnetic sensors}

Zhicheng Yang 
The research leading to this dissertation has received funding from China Scholarship Council (CSC). It is a joint research between Northwestern Polytechnical University (NPU) and University of Twente (UT) under the "Memorandum of agreement (MoA) for a double doctorate degree between NPU and UT". 


\title{
Hand-finger pose tracking using inertial and magnetic sensors
}

\author{
Dissertation \\ to obtain \\ the degree of doctor at the University of Twente, \\ on the authority of the rector magnificus, \\ Prof. dr. ir. A. Veldkamp, \\ on account of the decision of the graduation committee, \\ to be publicly defended \\ Thursday 24 February 2022 at 14:45 hours
}

by

\section{Zhicheng Yang}

born on the 11th of March, 1991

in Henan, Xuchang, China 
This dissertation has been approved by:

\author{
Supervisors \\ prof. dr. ir. P.H. Veltink \\ prof. dr. ir. Y. Shenggang \\ Co-supervisor \\ dr. B.J.F. van Beijnum
}

$\begin{array}{ll}\text { Cover design: } & \text { Zhicheng Yang } \\ \text { Printed by: } & \text { Ipskamp B.V. Enschede } \\ \text { Lay-out: } & \text { Zhicheng Yang } \\ \text { ISBN: } & 978-90-365-5333-9 \\ \text { DOI: } & 10.3990 / 1.9789036553339\end{array}$

(C) 2022 Zhicheng Yang, The Netherlands. All rights reserved. No parts of this thesis may be reproduced, stored in a retrieval system or transmitted in any form or by any means without permission of the author. Alle rechten voorbehouden. Niets uit deze uitgave mag worden vermenigvuldigd, in enige vorm of op enige wijze, zonder voorafgaande schriftelijke toestemming van de auteur. 


\section{Composition of the Graduation Committee:}

Chairman and secretary:

Prof. dr. J.N. Kok

University of Twente

Supervisor:

prof.dr.ir. P.H. Veltink

University of Twente

prof. dr. Y. Shenggang

Northwestern Polytechnical University

Co-supervisors:

dr. B.J.F. van Beijnum

University of Twente

Members:

prof.dr.ir. G.J.M. Krijnen

prof.dr.ir. M. Sartori

prof. dr. H.E.J. Veeger

University of Twente

prof. Dr.-ing. H. Vallery

University of Twente

TU Delft

dr.ing. P. Shull

TU Delft

Shanghai Jiao Tong University 


\section{Summary}

Hand-finger motion tracking during daily life is often used in rehabilitation for diagnostic and human-computer interaction etc. Traditionally, optical tracking systems (OTSs) are used. However, these systems are restricted to lab environments with expensive cameras and data acquisition systems. Ambulatory tracking out of a lab, using inertial sensors and magnetometers, is becoming increasingly popular to obtain insight in daily life.

There are two main disadvantages of the inertial and magnetometerbased tracking system. Firstly, the most existing methods are limited by high number of sensors, thus not satisfying the requirement of minimum obtrusiveness. Secondly, most existing methods require an accurate kinematic hand and finger model. From these two disadvantages, the goal of this thesis was derived: developing a minimally obtrusive inertial and magnetic sensing system that can be used in an ambulatory setting without kinematic information. The thesis was a joint work between Northwestern Polytechnical University (NPU) and University of Twente (UT), which has two parts. The first part was mainly about calibration of magnetometers, which provides basis for the follow-up work related to the localization with a magnetometer. This part was finished at NPU. The second part was to estimate the fingertip pose with few inertial sensors, magnetometers and a magnet, which was finished at UT.

The first part is addressed in Chapter 2 and 3. Chapter 2 presents a hybrid calibration method for the gradiometer or magnetometer array with more than two magnetometers. The first magnetometer was calibrated with 'scalar calibration' method, then errors from the magnetometer itself and misalignment error between magnetometers were calibrated together with linear method. The calibration efficiency can be greatly improved when the number of magnetometers is large. Chapter 3 presents a calibration method when the magnetometer is equipped on a carrier and its movement is restricted in a small range. When the magnetometer is restricted in a small range, the calibration data is insufficient and traditional calibration methods will fail. We exploited an improved truncated singular value decomposition method to obtain the error parameters and solved the divergent problem. The deviation of magnetometer output norm reduced a lot after calibration, which 
was verified when a magnetometer was fixed on a car.

The second part is addressed in Chapter 4, 5, 6 and 7. Chapter 4 presents a method that improves the OTS-based orientation estimation performance by fusing gyroscope information, providing a better orientation reference for following sections. The disadvantages of OTS-based orientation estimation are line-of-sight and wrong identification of mark problems. Moreover, when the tracking object is small, such as a fingertip, the orientation error can be large. The excellent dynamic performance of the gyroscope improves the orientation accuracy. The OTS-based orientation error was reduced from $0.39^{\circ} \pm 0.16^{\circ}$ to $0.23^{\circ} \pm 0.02^{\circ}$, when the distance between marker was $13 \mathrm{~mm}$. Besides, the proposed method filled the orientation data during 'line of sight' period and corrected the orientation estimates when OTS markers were wrongly identified. Chapter 5 presents a method to estimate fingertip orientation relative to the hand only with inertial sensors. 'designated event' (when the hand moves as whole object) was used to compensate the drift. During the 'designated event', the dorsal side of the hand and fingertips share approximate angular velocity and acceleration. The results showed, the orientation error was smaller than 10 degrees when the 'designated event' was partially available in a functional water-drinking task. Chapter 6 presented the position estimation of fingertips relative to the hand, with one magnetometer on the fingertip and one magnet on the dorsal side of the hand. We made assumptions that geomagnetic field is a disturbance compared with the magnet and the finger orientation relative to the hand is known. The finger position relative to the hand was estimated with Levenberg-Marquardt method. The experiment based on action research arm test resulted in median distance error between thumb and index finger of 9.6\%. Chapter 7 presents the results that fused method in chapter 5 and 6 . In chapter 7, the fingertip orientation was obtained based on inertial sensors with the method from chapter 5 , rather than the orientation from the OTS (used in Chapter 6). Compared with the orientation from the OTS, the orientation from the inertial sensors may contained larger errors. The experiment results show: For whole hand rotation and functional grasping or writing experiments, the estimated errors of index fingertip position and orientation relative to the hand were $8.0 \sim 9.8 \mathrm{~mm}$ and $5.7^{\circ} \sim 11.27^{\circ}$. 


\section{Samenvatting}

Meting en analyse van hand- en vingerbewegingen tijdens het dagelijks leven wordt vaak gebruikt in revalidatie voor bijvoorbeeld diagnostiek en mens-computer interactie. Traditioneel worden optische bewegingsmeetsystemen gebruikt (OTSs: optical tracking systems). Deze systemen zijn echter beperkt tot laboratoriumomgevingen en gebruiken dure camera's en dataacquisitiesystemen. Ambulante bewegingsmeting buiten het lab, gebruikmakend van inertiële en magnetische sensoren, wordt echter steeds vaker gebruikt om inzicht te krijgen in het menselijk bewegen tijdens het dagelijks leven.

Er zijn twee belangrijke nadelen van bewegingssensing met behulp van inertiële en magnetische sensoren. Allereerst, worden de meeste bestaande methoden beperkt door het gebruik van een groot aantal sensoren. Daardoor zijn ze relatief belastend voor de gebruiker. Ten tweede, vereisen de meeste bestaande methoden een nauwkeurig kinematisch hand- en vingermodel. De doelstelling van dit proefschrift relateert aan deze twee nadelen: het ontwikkelen van een ambulant bewegingsmeetsysteem voor de hand en vingers met inertiële en magnetische sensoren dat niet gebruik maakt van een kinematisch hand-vinger model en zo weinig mogelijk sensormodules bevat, waardoor het minimaal belastend voor de gebruiker kan zijn. Dit proefschrift is tot stand gekomen in het kader van een samenwerking tussen de Northwestern Polytechnical University (NPU) en de Universiteit Twente (UT), en bestaat uit twee delen. Het eerste deel gaat vooral over het kalibreren van magnetometers, en vormt de basis voor het vervolgonderzoek betreffende lokalisatie door middel van een magnetometer. Dit eerste deel van dit promotieonderzoek is gerealiseerd bij NPU. Het tweede deel betreft het schatten van hand-vinger houdingen en bewegingen doormiddel van enkele inertiële sensoren en magnetometers, en een permanente magneet. Dit tweede deel van het onderzoek is verricht aan UT.

Het eerste deel wordt behandeld in Hoofdstukken 2 en 3. Hoofdstuk 2 presenteert een hybride kalibratiemethode voor een gradiometer of magnetometerarray met meer dan twee magnetometers. De eerste magnetometer werd gekalibreerd met een scalaire kalibratiemethode. Vervolgens werden fouten van de magnetometer zelf en uitlijningsfouten tussen magnetometers 
gekalibreerd met een lineaire methode. De kalibratie-efficiëntie kan aanzienlijk worden verbeterd wanneer het aantal magnetometers groot is. Hoofdstuk 3 presenteert een kalibratiemethode voor de situatie dat de magnetometer is gepositioneerd op een drager en de beweging ervan beperkt is binnen een klein bereik. Onder deze conditie zijn de meetgegevens onvoldoende voor volledige kalibratie en zullen traditionele kalibratiemethoden mislukken. We hebben het bijbehorende divergentieprobleem opgelost doormiddel van een verbeterde methode voor afgeknotte singuliere waarde decompositie. De afwijking van de norm van de magnetometer uitgang nam beduidend af na toepassing van deze kalibratiemethode. Dit is geverifieerd met een magnetometer die op een auto was bevestigd.

Het tweede deel wordt behandeld in Hoofdstukken 4, 5, 6 en 7. Hoofdstuk 4 presenteert een methode die de OTS-gebaseerde oriëntatieschatting verbetert door fusie met metingen van een hoeksnelheidssensor. Dit resulteerde in een verbeterde oriëntatiereferentie voor de studies in de volgende hoofdstukken. De problemen die optreden bij oriëntatieschatting met OTS zijn verstoring van de zichtlijn en incorrecte identificatie van optische markers. Bovendien resulteert de OTS methode in relatief grote fouten in het schatten van oriëntaties van kleine objecten, zoals een vingertop. De uitstekende dynamische prestaties van de hoeksnelheidssensor verbeteren de nauwkeurigheid van deze oriëntatieschatting. De op OTS gebaseerde oriëntatiefout werd teruggebracht van $0.39^{\circ} \pm 0.16^{\circ}$ zonder tot $0.23^{\circ} \pm 0.02^{\circ}$ met fusie met hoeksnelheidsinformatie bij een afstand van $13 \mathrm{~mm}$ tussen de markers. Bovendien vulde de voorgestelde fusiemethode de oriëntatieschatting tijdens periodes waarin de zichtlijn was verstoord en corrigeerde de fusiemethode de oriëntatieschattingen wanneer OTS-markers verkeerd werden geïdentificeerd. Hoofdstuk 5 presenteert een methode om de vingertoporiëntatie ten opzichte van de hand te schatten, met alleen inertiële sensoren. Voor dit doel werden tijdintervallen waarin de hand met vingers als geheel object beweegt zonder noemenswaardige verandering van relatieve oriëntaties en posities ('designated events') gebruikt om de drift te compenseren. Tijdens deze 'designated events' werd verondersteld dat de dorsale zijde van de hand en de vingertoppen dezelfde hoeksnelheid en versnelling ondergaan. De resultaten toonden aan dat de oriëntatiefout kleiner was dan $10^{\circ}$ wanneer zulke 'designated events' voor een deel van de tijd aangewezen waren tijdens een functionele waterdrinktaak. Hoofdstuk 6 presenteert de positiebepaling van de vingertoppen ten opzichte van de hand, met een magnetometer op de vingertop en een magneet op de dorsale zijde 
van de hand. We hebben het aardmagnetisch veld als een verstoring op het veld van de magneet beschouwd, en aangenomen dat de relatieve oriëntatie tussen vingertop en de hand bekend is. De relatieve positie tussen vingertoppen en hand is geschat behulp van de Levenberg-Marquardt-methode. Het experiment gebaseerd op de action research arm test resulteerde in een mediane relatieve afstandsfout tussen duim en wijsvinger van 9.6\%. Hoofdstuk 7 combineert de methoden uit hoofdstuk 5 en 6 . De oriëntatie van de vingertop werd daarbij niet met een OTS geschat, maar doormiddel van inertiële sensoren, zoals gepresenteerd in hoofdstuk 5. Dit resulteerde in verschillen in oriëntatieschatting. De resultaten van het experiment laten zien: Voor rotatie van de hele hand en functionele schrijf- of grijpexperimenten waren de geschatte fouten van de positie en oriëntatie van de wijsvingertop ten opzichte van de hand 8.0 respectievelijk $9.8 \mathrm{~mm}$ en $5.7^{\circ}$ respectievelijk $11.3^{\circ}$. 


\section{Contents}

$\begin{array}{ll}\text { Summary } & \text { v }\end{array}$

Samenvatting (Summary in Dutch) vii

1 General introduction 1

2 Hybrid Calibration Method for Three-axis Gradiometer 17

3 Calibration of tri-axis magnetometers based on improved truncated singular value decomposition method

4 Improvement of optical tracking-based orientation estimation by fusing gyroscope information

5 Estimation of relative hand-finger orientation using a small IMU configuration

6 Estimate Hand-finger position with one magnetometer and known relative orientation

7 Hand-finger pose estimation using few inertial and magnetic sensors and a magnet

8 General discussion and conclusion

Bibliography

Acknowledgement

About the author

List of publications 


\section{General introduction}


1 General introduction

\section{General introduction}

Accurate and minimally obtrusive 3D tracking of hand and finger pose during daily-life is relevant for several important classes of applications, but has not been adequately solved to-date. This thesis presents and evaluates new sensing and analysis concepts that combine inertial and magnetic sensing with the objective to contribute to this important field.

This general introduction first presents motivations for further investigating 3D tracking of hand and finger pose during daily-life, and presents current modalities that can be used in and outside of the lab and their limitations (section 1.1). Subsequently, this introduction provides an overview of 3D motion tracking of the hand using inertial measurement units (IMU) (section 1.2), magnetic sensing (section 1.3), and the fusion of both (1.4). Finally, the objectives of this $\mathrm{PhD}$ research (section 1.5) and the organization of the current thesis (section 1.6) are presented.

\subsection{Motivation for investigating improved sensor- based 3D tracking of hand and finger pose}

Hand and finger pose tracking in 3D during daily-life reflects human health and performance as well as the interaction with the environment. Such 3D tracking is currently only possible in well-equiped movement laboratories, and has important limitations. Methods for accurate and minimally obtrusive sensing of 3D hand and finger pose tracking under daily-life conditions are currently not available.

First, I will present representative applications of 3D hand and finger pose tracking in daily-life. Subsequently, I will introduce optical 3D movement analysis in the laboratory, which is the current standard. Finally, I will present sensing modalities for daily-life assessment of 3D hand and finger pose tracking and their current limitations.

\section{Applications of 3D hand and finger pose tracking}

Hand motion tracking is important for a wide range of applications, including human-computer interaction, sports, virtual reality and rehabilitation: 
1.1 Motivation for investigating improved sensor-based 3D tracking of hand and finger pose

- Human-computer interface: Human-computer interaction has become more and more important in our daily lives. The traditional interaction methods, such as mice, keyboards and touch screens become increasingly unable to meet the requirements of friendly interaction. In comparison, the movement of hand and fingers can provide a more natural and friendly way to interact between the human and computer $[1,2]$.

- Sports: in sports, the coach often gives initial instructions, subsequently evaluate the motion of players, and finally provides corrective feedback. Hand motion tracking is important for coach in sports related to the hand, such as golf, ball games etc $[3,4,5]$.

- Virtual reality: For augmented reality (AR) and virtual reality (VR) applications, virtual objects are rendered in a real world context with accurate poses and poses of users are also need to be known in such a world. Thus, calculating poses of user hands accurately is one of the most important issues in AR/VR [6,7].

- Rehabilitation: For patients who have suffered a stroke or other motor function disability, the movements of patients need to be monitored and trained such that they relearn a functional movement pattern, which is is clinically important $[8,9,10]$, including the hand.

A common task for all applications above is to measure hand movement in an interpretable and ambulatory way to users. Due to the flexibility and complexity of hand movement, the task for accurate and minimally obtrusive hand movement tracking under ambulatory conditions is difficult and there is no generally acknowledged best way to achieve that. Currently, 3D optical tracking methods are the standard in the lab. Under ambulatory conditions, on-body tracking modalities have been developed. Among them, the lab-based 3D tracking method can be divided into marker-based, markerlessbased and electromagnetic field (EF)-based tracking method. The on-body tracking methods include Mechanical-based tracking, Flexible sensor-based tracking, inertial and magnetic measurement unit (IMMU)-based tracking.

\section{Lab-based 3D tracking of hand and fingers}

The lab-based 3D tracking method can be divided into marker-based, markerlessbased and electromagnetic field (EF)-based tracking method. 
Marker-based optical tracking: The marker-based optical tracking system (OTS) consists mainly of high-speed cameras and markers [11]. The high-speed camera determines the distance between the marker and the camera by capturing the infrared rays emitted by the marker ball. The advantage of using infrared rays is that it can filter out the interference of natural light. According to whether the marker can emit light, markers can be divided into active markers and passive markers. The active marker is an infrared LED light. The passive marker is a special ball coated with a luminescent material, which can reflect infrared light well. Active markers are generally larger in volume than passive markers due to the need for auxiliary devices such as power supply. The advantage of the marker-based optical tracking method is that it has high accuracy, high sampling rate. However, its disadvantages are high prices, line of sight problem etc. Thus, it is often used in fields that require high accuracy but insensitive to price, such as medical and scientific research fields.

Marker-based optical hand and finger tracking is very challenging, even in the lab: line of sight between cameras and markers is often interrupted during natural arm, hand and finger movements. In addition, 3D orientation of finger segments and joints is difficult to estimate even under good line of sight conditions. This is related to movement of markers with the skin relative to finger joints during functional finger movements and non-ideal joint characteristics [12].

Markerless-based optical tracking: Compared with marker-based optical tracking, the markerless-based optical tracking only exploits camera but without any marker [13]. Generally, this method can be divided into two categories: one exploits RGB cameras [13] and the other one exploits depth cameras [14]. With a RGB camera, we can obtain a 2D image of the target, and then use the 2D image information of multiple cameras to estimate the three-dimensional position of the target. Since the image contains lots of redundant information, the processing is time-consuming. In addition, real-time tracking requires hardware with higher performance, and it is difficult to achieve high speed localization. With depth cameras, we can obtain depth information, which can be used to estimate the position of the target. However, this method can only cover a small area and is often applied close to the cameras. 
1.1 Motivation for investigating improved sensor-based 3D tracking of hand and finger pose

Due to the limited working space, line-of-sight problem etc [12]., these two optical tracking methods are not satisfying ambulatory condition.

EF-based tracking: The EF tracking method realizes target positioning through low-frequency electromagnetic transmitting and receiving devices $[15,16]$. Compared with the optical tracking system, the EF-based tracking has no line-of-sight problem. However, EF-based tracking system can be easily interfered by surrounding ferromagnetic materials. When the tracking targets increase, the system needs more transmitting equipment, including different signal sources and amplifiers [16]. Hand-finger tracking based on EF is expected to be applied in the field of virtual reality [16].

\section{On body tracking of hand and fingers}

The on body tracking of hand and finger methods include mechanical-based tracking, flexible sensor-based tracking, inertial and magnetic measurement unit (IMMU)-based tracking.

Mechanical-based tracking: The mechanical tracking system is one of the earliest tracking systems, which is similar to the exoskeleton wearable system, mainly composed of rigid mechanical mechanisms and joints [17]. The movement of the hand drives the rotation of the mechanical joints. Combined with the length of the rigid mechanism, the poses of different joints can be estimated. The device has a simple principle and low requirements for the environment, but it may hinder the user's activities to a certain extent, and its accuracy is low [18]. It is often used in some tracking occasions combined with haptic feedback control [17].

Flexible sensor-based tracking: The flexible sensors used for hand motion tracking can be divided into three categories: (1) flexible sensors based on conductive ink [19,20]; (2) flexible sensors based on optical fibers [21, 22]; (3) flexible sensors based on conductive fabric/wire /polymer [23]. Take the conductive ink-based flexible sensor as an example, it has a certain resistance when it is placed horizontally, and when it is bent, the corresponding resistance will change. When the finger is bent, the impedance of the flexible sensor will change. 
1 General introduction

Based on this change, the degree of bending of the finger can be estimated. This method has the advantages of simple structure, low price and commonly used for entertainment games [24].

IMMU-based tracking: Compared with the mentioned tracking methods above, the hand motion tracking based on IMMU is a new method developed in recent years [25, 26]. A unit in tracking system includes an acceleration sensor, a gyroscope, and a magnetometer. The advantage of IMMU-base hand tracking lies in its small size and low power consumption, making it possible to wear. At the same time, the price of IMMU-based tracking devices is low [25]. However, an IMMU is required on each finger segment, resulting in undesired obtrusiveness by the users, and accuracy is limited by using kinematic hand and finger model [12].

Compared with other motion tracking systems, the most attractive advantage of IMMU-based motion tracking is that ambulatory assessments can be performed out of lab environments [27, 28]. Moreover, it can directly measure acceleration and angular velocity. Based on the raw data, orientation can be obtained by integrating the angular velocity provided with initial orientation. Owing to the drift errors, change of orientation can not be estimated accurately for periods longer than dozens of seconds. Fortunately, drift issues can be solved by fusing the data from accelerometers and magnetometers. However, the most advanced current methods that have been published are limited by high number of sensors, thus not satisfying the requirement of minimal obtrusiveness and the use of an inaccurate kinematic hand and finger model. Therefore, new steps need to be made in the development of minimally obtrusive inertial and magnetic sensing system that can be used in an ambulatory setting.

In the following sections, motion tracking using inertial and magnetic sensor modules (section 1.2), position tracking using a magnetic positioning system (section 1.3) and the fusion between both approaches (section 1.4) will be presented. They are the basis for the 3D hand and finger tracking concept that have been developed and evaluated in the current $\mathrm{PhD}$ research, which is reported in this thesis. 


\subsection{Motion tracking of the hand based on IMMUs}

From the literature, the traditional IMMU-based hand tracking system includes an IMMU attached to each segment $[25,29]$. The orientation of each segment expressed in global frame can be estimated with the corresponding IMMU. The position of each segment can, subsequently, be estimated with forward kinematics, estimated orientation and segments' lengths [26].

\subsubsection{Orientation estimation with IMMUs}

A variety of studies have been conducted on the orientation estimation based on IMMUs [30, 31, 32, 33]. Based on the angular velocity measured by the gyroscope, the orientation change can be calculated as

$$
\boldsymbol{q}_{s, k-1}^{s, k}=\left[\begin{array}{ll}
1 & \frac{1}{2} \boldsymbol{\omega}_{s, k} t
\end{array}\right]
$$

where $\boldsymbol{q}_{s, k-1}^{s, k}$ is the orientation change expressed in quaternion. $\boldsymbol{\omega}_{s, k}$ is the angular velocity at time time $k$ in sensor frame. The orientation relative to the global frame can be updated as

$$
\boldsymbol{q}_{s, k}^{g}=\boldsymbol{q}_{s, k-1}^{g} \otimes \boldsymbol{q}_{s, k-1}^{s, k}
$$

where $\boldsymbol{q}_{s, k}^{g}$ is the orientation respect to the global frame. However, the orientation integrated by the gyroscope can only be trusted during a short period because of the drifts. When the non-gravitational acceleration is negligible compared with the gravity, the accelerometer output $\boldsymbol{y}_{a c c, k}^{s}$ can be expressed as

$$
\boldsymbol{y}_{a c c, k}^{s}=\left(\boldsymbol{q}_{s, k}^{g}\right)^{-1} \otimes\left[\begin{array}{ll}
0 & \boldsymbol{g}_{g}^{g}
\end{array}\right] \otimes\left(\boldsymbol{q}_{s, k}^{g}\right)
$$

where $\boldsymbol{g}_{g}^{g}$ is the local gravity in global frame. When the magnetic disturbance is negligible compared with the geomagnetic field, the magnetometer output $\boldsymbol{y}_{\text {mag, }, k}^{s}$ can be expressed as

$$
\boldsymbol{y}_{m a g, k}^{s}=\left(\boldsymbol{q}_{s, k}^{g}\right)^{-1} \otimes\left[\begin{array}{ll}
0 & \boldsymbol{B}_{g}^{g}
\end{array}\right] \otimes\left(\boldsymbol{q}_{s, k}^{g}\right)
$$

where $\boldsymbol{B}_{g}^{g}$ is the geomagnetic field in global frame. Based on Eq (1.3) and (1.4), the orientation drift can be well compensated. When the orientation of each segment is known in the global frame, the relative orientation between finger segments can be easily obtained. 


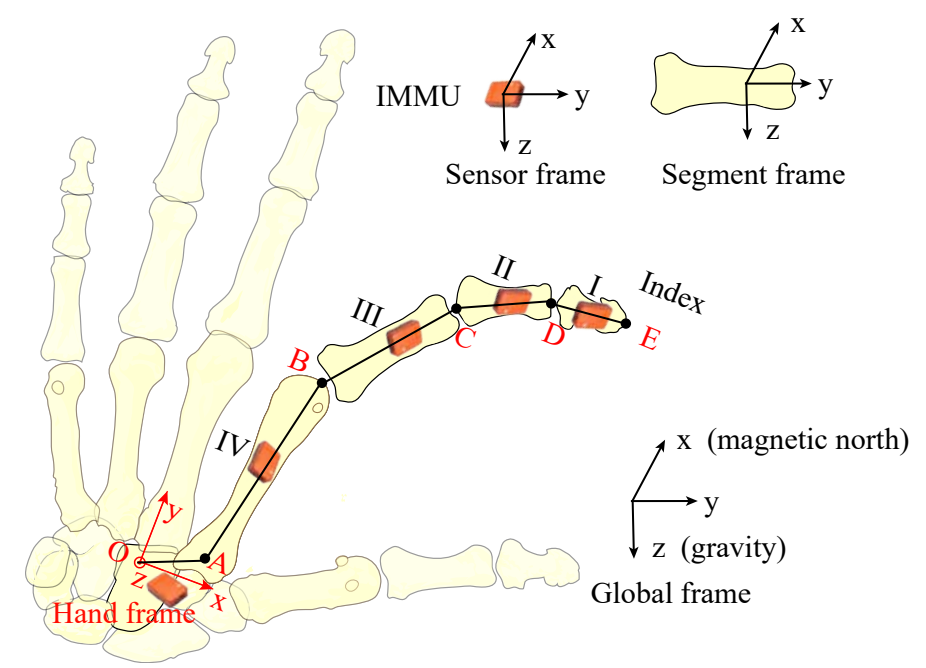

Figure 1.1: Illustration of hand anatomy attached with IMMUs. Based on the information of IMMUs and lengths of segments, the position and orientation of fingertip can be estimated [25].

One of the biggest challenges for the orientation estimation based on IMMU is the magnetic disturbance $[34,35]$. As many activities are performed in indoor environment, there are many ferromagnetic objects around. Thus, the orientation estimation can be easily disturbed. In order to overcome magnetic disturbances, some assumptions were proposed that some joints can only rotated in $2 \mathrm{DoF}$. In this way, the magnetometer can be neglected. However, thumb joints have more degrees of freedom and their rotations cannot be simplified as $2 \mathrm{DoF}$.

\subsubsection{Position estimation with inertial sensors}

Based on the strapdown navigation, change of position can be obtained by a double integral of non-gravitational accelerations extracted from the IMMU after transformation to the global frame using a 3D orientation estimate. However, the extracted non-gravitational acceleration often contains errors and the double time integration of acceleration results in extensive integration drift within seconds $[36,37,38]$. The drift can be larger than the length of fingers, which is not suitable for hand-finger position estimation. Alternatively, the finger position relative to the hand can be estimated using the forward kinematics with the known orientation and length of each segment 
$[25,26]$. As shown in Fig 1.1, except for the thumb, each finger contains 4 phalanges and each phalanx is considered rigid. Based on the IMMU attached on phalanx, the orientation of each phalanx in global frame is known. Then the relative orientation between adjacent phalanges is known. According to the forward kinematic chain rule and the length of phalanges, the fingertip position relative to the dorsal side of the hand can be expressed as

$$
\boldsymbol{p}_{E}=\boldsymbol{l}_{O A}^{h}+\boldsymbol{q}_{s e g 4}^{h} \otimes\left(\boldsymbol{l}_{A B}^{\text {seg } 4}+\boldsymbol{l}_{B C}^{\text {seg } 4}+\boldsymbol{l}_{C D}^{\text {seg } 4}+\boldsymbol{l}_{D E}^{\text {seg } 4}\right) \otimes\left(\boldsymbol{q}_{\text {seg }}^{h}\right)^{*}
$$

where $l_{B C}^{\text {seg } 4}, l_{C D}^{\text {seg } 4}, l_{D E}^{\text {seg } 4}$ can be expressed as

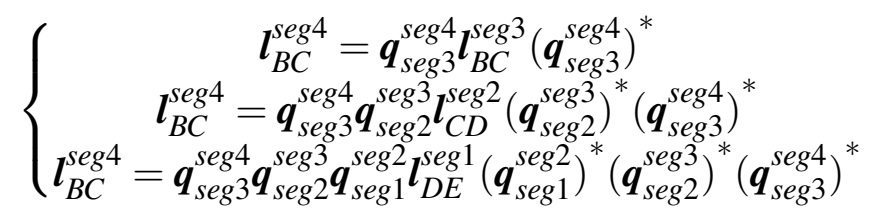

The challenges of position estimation using forward kinematics are: each segment needs to wear an IMMU and its length needs to be measured for every participant. Moreover, the measurement error of segment length and estimated orientation error accumulate with the forward kinematic. Besides, Models using forward kinematics assume rigid segments and ideal joints, which is not the case in reality [12]. In order to solve these problems, we introduce a magnet as a passive magnetic source in this PHD research, together with the magnetometer, a passive localization system was formed, which can estimate the fingertip position without forward kinematics.

\subsection{Position tracking with a magnetic localization system}

The magnetic localization system has the advantage of high accuracy, no line-of-sight problem etc., which is a good compensation for inertial-based position system in many biomedical applications. Yongde et al. proposed a intraoperative organ motion tracking method by fusing IMMU and electromagnetic system [39]. The effect of magnetic disturbance is greatly reduced. Roetenberg et al. proposed a method for ambulatory pose estimation by fusing IMMUs and orthogonal coils worn on the body [40].

Compared with the fusion of ultrasound systems, EF tracking system provides position information instead of distance information, which is more 
1 General introduction

suitable for the compensation of position drift. However, drawbacks still exist. Firstly, the body-worn EF tracking system is bulky, which may hinder movements of subjects. Secondly, it consumes large amounts of energy, especially when the EF tracking system needs to cover a large tracking volume. Thirdly, it may be disturbed by magnetic disturbances that vary over place and time [41].

To overcome drawbacks of EF tracking system, a potential alternative is to exploit a magnet as a passive magnetic emitter instead of active magnetic coils $[42,190]$. Compared with orthogonal coils, the advantages of the magnet are that it has smaller volume and no power assumption. The disadvantages are that the magnetic field generated by the magnet is mixed with geomagnetic field, which cannot be easily extracted. Thus the system requires more information to achieve the localization.

\subsubsection{Calibration of magnetometers}

The magnetometer output is influenced by many different kinds of errors, which may influence the position accuracy. Therefore, the magnetometer need to be calibrated before use. The magnetometer errors can be divided into two categories, one is caused by the magnetometer itself and the other one is caused by its carriers. The magnetometer output $\boldsymbol{y}_{m a g}^{s}$ can be expressed as

$$
\begin{aligned}
\boldsymbol{y}_{\text {mag }}^{s} & =\boldsymbol{A}_{\text {soft }} \boldsymbol{A}_{\text {non }} \boldsymbol{A}_{\text {sen }}\left(\boldsymbol{B}_{m}^{s}+\boldsymbol{b}_{\text {off }}+\boldsymbol{b}_{\text {hard }}\right)+\boldsymbol{n}_{B} \\
& =\boldsymbol{A}\left(\boldsymbol{B}_{m}^{s}+\boldsymbol{b}\right)+\boldsymbol{n}_{B}
\end{aligned}
$$

where $\boldsymbol{A}_{\text {sen }}, \boldsymbol{b}_{\text {off }}$ and $\boldsymbol{A}_{\text {non }}$ are sensitivity error, offset error and non-orthogona -lity error, which come from the magnetometer. $\boldsymbol{b}_{\text {hard }}$ and $\boldsymbol{A}_{\text {soft }}$ are hard-iron effect error and soft-iron effect error, which come from the carrier. $\boldsymbol{B}_{m}^{s}$ is the magnetic field to be measured in magnetometer frame. $\boldsymbol{n}_{B}$ is the measurement noise. $\boldsymbol{A}_{\text {soft }}, \boldsymbol{A}_{\text {non }}$ and $\boldsymbol{A}_{\text {sen }}$ are often estimated as an integrated error parameter $\boldsymbol{A}$. $\boldsymbol{b}_{\text {off }}$ and $\boldsymbol{b}_{\text {hard }}$ are estimated as $\boldsymbol{b}$.

The state-of-art calibration methods exploit the strength of the geomagnetic field as a reference. When only the geomagnetic field is measured, which could be done in outdoor environment, $\boldsymbol{B}_{m}^{s}$ can be expressed as

$$
\boldsymbol{B}_{m}^{s}=\boldsymbol{q}_{g}^{s} \otimes\left[\begin{array}{ll}
0 & \boldsymbol{B}_{g}^{g}
\end{array}\right] \otimes\left(\boldsymbol{q}_{g}^{s}\right)^{*}
$$

During a rotational movements, $\boldsymbol{q}_{g}^{s}$ is unknown. However, $\left\|\boldsymbol{B}_{m}^{s}\right\|_{2}$ is known 
as the strength of the local geomagnetic field. With enough measurements at different orientations, parameter $\boldsymbol{A}$ and $\boldsymbol{b}$ can be estimated.

\subsubsection{Localization with a dipole model}

After calibration, the magnetometer data is ready for the localization of the magnet. When a ferromagnetic object is far from the sensor, the object can be approximated as a dipole [43]. The magnet is often approximated as a dipole model during the localization $[44,45]$. As shown in Fig 1.2, the

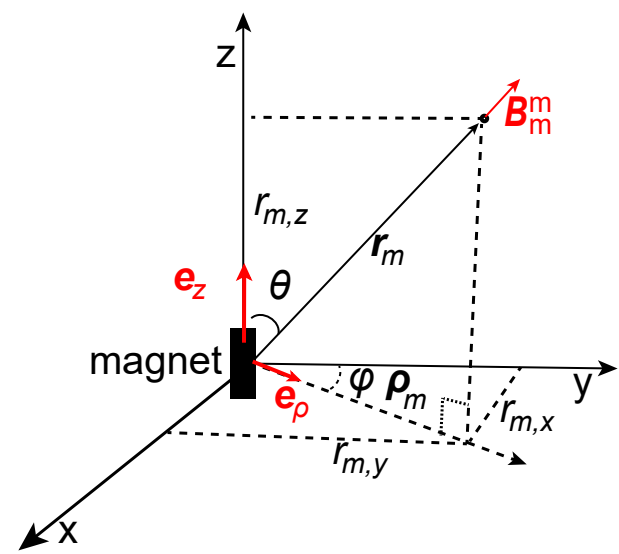

Figure 1.2: Dipole model of a magnet. The coordinate system is centered at the center of the magnet, and the z-axis is chosen along the magnetic axis. The magnetic field at position $\boldsymbol{r}_{m}$ is $\boldsymbol{B}_{m}$.

magnetic field at $\boldsymbol{r}_{m}$ generated by the magnet (located at the origin) is

$$
\boldsymbol{B}_{m}^{m}=\frac{\mu_{0} M_{m}}{4 \pi r_{m}^{3}}\left[\frac{3}{2} \sin 2 \theta \boldsymbol{e}_{\rho}+\left(3 \cos ^{2} \theta-1\right) \boldsymbol{e}_{z}\right]
$$

where $\mu_{0}$ is the permeability of free space and $M_{m}$ is the magnetic moment. $\boldsymbol{r}_{m}$ is the position in the magnet frame, expressed as follows in a spherical coordinate system:

$$
r_{m, x}=r_{m} \cos \theta \sin \varphi, r_{m, y}=r_{m} \cos \theta \cos \varphi, r_{m, z}=r_{m} \sin \theta
$$

where $\left(r_{m}, \theta, \varphi\right)$ gives the radial distance, polar angle, and azimuthal angle. $\boldsymbol{e}_{\rho}$ and $\boldsymbol{e}_{z}$ are projections of $\boldsymbol{r}_{m}$ on the horizontal plane and vertical axis, as shown in Fig 1.2. 
1 General introduction

Suppose the magnetic field generated by the magnet is measured by the magnetometer as $\boldsymbol{B}_{m}^{s}$, which is in the magnetometer frame. The relative orientation between magnet frame and magnetometer frame $\boldsymbol{q}_{s}^{m}$ is also unknown. One magnetometer is not enough to estimate orientation $\boldsymbol{q}_{s}^{m}$ and position $\boldsymbol{r}_{m}$ simultaneously. More information is needed if $\boldsymbol{r}_{m}$ is to be estimated. Still, magnetometer outputs contains important position information.

\subsection{Data fusion of inertial sensor and magnetome- ter}

In the $\mathrm{PhD}$ research reported in this thesis, we propose a small and potentially relative unobtrusive IMMU setup combined with a permanent magnet. The proposed sensor configuration includes two IMMUs attached to the most distal segments of thumb and index finger, and one IMMU with a permanent magnet attached to the dorsal side of the hand, as shown in Fig 1.3.

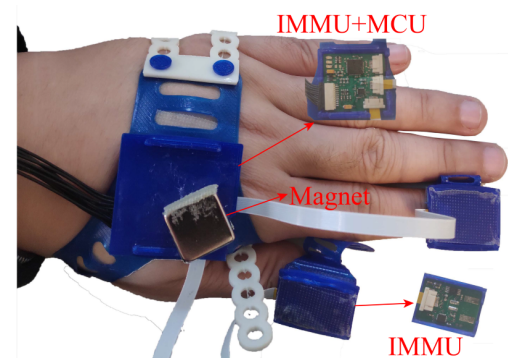

Figure 1.3: Experimental setup of a potentially low obtrusive sparse IMMU and permanent magnet configuration for estimating fingertip poses relative to the dorsal side of the hand, which is applied in the research on new concepts for $3 D$ hand and finger tracking that are presented and evaluated in this PhD thesis.

As described above, IMMUs are good tools for estimating hand-finger orientations, but not the hand-finger position. Meanwhile, a passive magnetic object localization system can provide relative position and orientation information which could be a good supplement. 


\subsection{Research objectives and problems}

In this thesis, we are concentrating on the rehabilitation applications with hand motion tracking, where the poses of thumb and index finger relative to the hand are important indicators.

Research objective: The objective of this thesis is to accurately estimate the pose of thumb and index finger relative to the back of the hand with a setup containing a low number of IMMUs, which may lead to a sensing system of low obtrusiveness in future.

We only retain the IMMUs at the tip of the thumb and index finger and the back of the hand. The lack of information about middle phalanx makes the chain rule for estimating the position of the fingertips invalid. Therefore, we add a magnet to the system as a passive emission source, and a magnetic sensor at the fingertip as a receiver for estimating the position of fingertip. Compared with the traditional system, sensors on the middle phalanxes are omitted, the reliance on assumed segment length and finger joint characteristics is eliminated, and the practicability of the device is greatly increased. Moreover, this approach is potentially applicable also in patients that have deformed hands and fingers due to for example osteoarthritis. However, the proposed system facing two main problems, these problems are:

Research problems: (1) The introduction of magnets greatly disturbs the geomagnetic field. Generally, the measurement of geomagnetic field by a magnetometer can compensate yaw direction drifts estimated by the gyroscope. When the magnetic field generated by the magnet is much larger than the geomagnetic field, it is impossible to perform the heading compensation using the magnetometer. How can we estimate the orientation of the fingertip when the geomagnetic field is heavily disturbed? (2) Due to the limited area of the fingertip, only one IMMU is placed, which contains one magnetic sensor. Generally, the real-time position estimation of a magnetic dipole requires two or more magnetometers. The insufficient number of magnetometers makes position estimation difficult. Can we accurately and reliably estimate the fingertip position with one magnetometer and magnet?

In order to solve the above problems, this thesis proposes new attitude and position estimation methods. The specific content of the current $\mathrm{PhD}$ thesis in relation to the research goal and problems presented above is introduced in the section 1.6. 


\subsection{Organization of the thesis}

This thesis is a joint work between University of Twente (UT) and Northwestern Polytechnical University, China (NPU). The introduction of a passive magnetic localization system is an important innovation of this thesis. In order to obtain good position performance, the magnetometer needs to be well calibrated. This is the topic presented in the first part of this thesis. The involved research was performed at NPU. The second part of this thesis presents the research performed at UT concerning the estimation of pose of finger tips relative to the back of the hand. All six chapters have been published as journal articles. The content of the subsequent chapters of this thesis are shown in Fig 1.4.

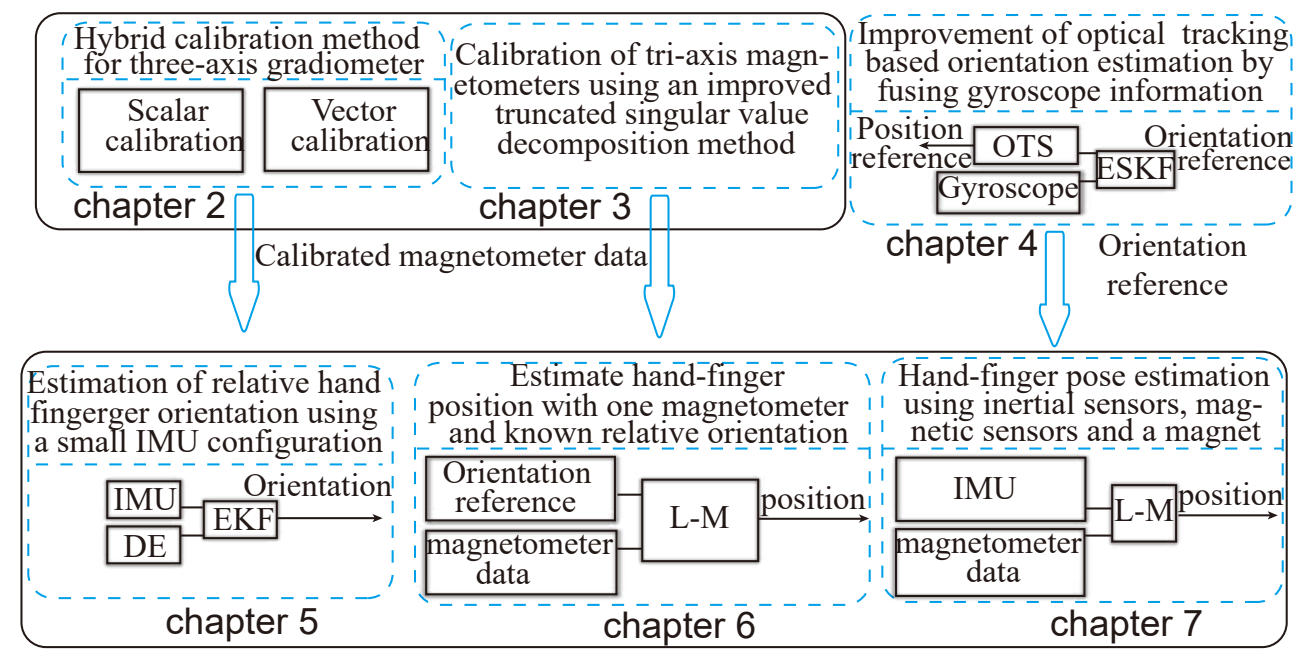

Figure 1.4: Algorithm for the estimating fingertip poses relative to the hand with IMMUs and a magnet. The abbreviations are: Error state Kalman filter (ESKF); designated event (DE); Extended Kalman filter (EKF); Levenberg-Marquardt (L-M).

\section{Part I. Calibration of magnetometers}

Chapter 2. Hybrid Calibration Method for Three-Axis Gradiometer.

We propose to calibrate the first magnetometer with a scalar calibration method, then calibrate the remaining magnetometers with a vector calibration method. In this way, the magnetometer error and misalignment error 
can be compensated simultaneously and the calibration efficiency can be improved.

Chapter 3. Calibration of tri-axis magnetometers using an improved truncated singular value decomposition method.

This chapter propose a novel method to calibrate magnetometers when the measurement data is not sufficient.

\section{Part II. Estimation of fingertip poses relative to the hand}

Chapter 4. Improvement of optical tracking-based orientation estimation by fusing gyroscope information. The orientation estimated by the optical tracking system has relatively large error but does not accumulate over time. In contrast, the orientation from a gyroscope is accurate in a short time horizon but accumulates over time. This chapter proposed a method fusing both to get a better performance for orientation estimation.

Chapter 5. Estimation of relative hand-finger orientation using a small IMU configuration. When the hand and fingertips move together, they experience almost the same angular velocities and accelerations. This chapter proposed a method to estimate fingertip orientation by using this information and IMUs.

Chapter 6. Estimate Hand-finger position with one magnetometer and known relative orientation. This chapter describes and evaluates a method to estimate finger position relative to the hand with one magnetometer on a fingertip and a permanent magnet on the dorsal side of the hand, assuming that the relative orientation is known.

Chapter 7. Hand-finger pose estimation using few inertial and magnetic sensors and a magnet. In chapter 6, the fingertip orientation relative to the dorsal side of the hand is from an OTS and contain negligible errors. In this chapter, the relative orientation is obtained using IMUs on hand and fingers (chapter 5), and thus contains errors, which is related to the types of hand movements. The performance of fingertip position estimation method, presented in chapter 6 , is investigated using the relative orientation estimation method of chapter 5 .

Chapter 8. General discussion and conclusion. This chapter concludes and discusses the the new concepts presented and evaluated in this thesis. Subsequently, it presents our view on future research directions. 
CHAPTER 2

\section{Hybrid Calibration Method for Three-axis Gradiometer}

This chapter is published as a journal article: Yang, Z., Yan, S., \& Li, B. (2017). Hybrid calibration method for three-axis gradiometer. IEEE Magnetics Letters, 8, 1-5. 


\title{
Hybrid calibration method for three-axis gradiometer
}

\begin{abstract}
The performance of a three-axis gradiometer (TAG) is limited by measurement and misalignment errors of two three-axis magnetometers (TAMs). We describe a simplified calibration method for TAGs. One TAM is calibrated with a scalar method. Self-errors of the other TAM and misalignment errors between the two TAMs are then calibrated as integrated errors based on a vector calibration. The experimental results show that the scalar output errors of the two TAMs and the vector output errors of the TAG are greatly reduced and the performance of the TAG is improved dramatically.
\end{abstract}




\subsection{Introduction}

The three-axis gradiometer (TAG) is composed of several three-axis magnetometer (TAM)s. Compared with a single TAM, TAG can not only provide the components of the vector field, but the gradient of the magnetic field which can suppress background interference to some extent [46, 47]. In this paper, we focus on a gradiometer composed of two TAMs on a stable support beam. Although it can only determine three components of the gradient tensor, it can be manufactured as a portable battery powered equipment which are widely used in archaeological survey, magnetic compensation in space, unexploded ordnance (UXO) detection [48, 49, 50]. However, the gradient outputs of the TAG are sensitive to the orientations even in the uniformly constant field, the inconsistent outputs are caused by self-errors from two TAMs and the misalignment errors between them, calibration is specifically of concern in precise TAG measurement systems [51]. In previous decades, more attention is drawn to calibrate the TAM. The calibration methods for TAM can be divided into two categories, vector calibration method and the scalar calibration method. For the traditional vector calibration method, expensive magnetic field devices or orientation instruments are needed to provide the reference magnetic field, the TAM is calibrated by comparing the outputs of itself with the reference magnetic field. Compared with the vector calibration method, only a homogenous magnetic field is needed in the scalar calibration method, because the geomagnetic field can be reckoned as constant and homogenous, scalar calibration method is more widely used than the vector calibration method $[52,53]$. The scalar calibration method is first proposed by Merayo et al with linear least squares algorithm to estimate the error parameters [54]. C. C. FOSTER expanded Merayo's research by adding the nonorthogonal error to the model [55], the advantages of Merayo and C. C. FOSTER's methods lie in that they can find unique parameters for a given data, meanwhile, they do not require iterations. After that, lots of methods are proposed to improve Merayo's method, such as the nonlinear calibration method [56, 57, 58], recursive fitting calibration method [59], mounting frame calibration method [60], adaptive least squares calibration method [61], total least squares calibration method [62, 63], unscented filter formulation method et al [64].

Compared with the TAM, few works were done on calibrating the TAG. Steven Turner et al proposed a vector calibration method for the TAG, some devices including gradient coils were used to generate the gradient magnetic 
field, the calibration parameters were estimated by comparing outputs of the TAG with the generated magnetic field, besides, the shielding techniques were used. However, this method has difficulties that high-precision devices which generate gradient magnetic field are hard to obtain [65]. There are several scalar calibration methods proposed to calibrate the TAG [66, 67, 68, $69,70]$, in these methods, the algorithms to estimate the error parameters may be a little different, but the calibration process can be divided into two steps, the first step is to calibrate two TAMs separately with scalar calibration method, the second step is to calibrate the misalignment errors between two TAMs, the process is shown in Fig 2.1.

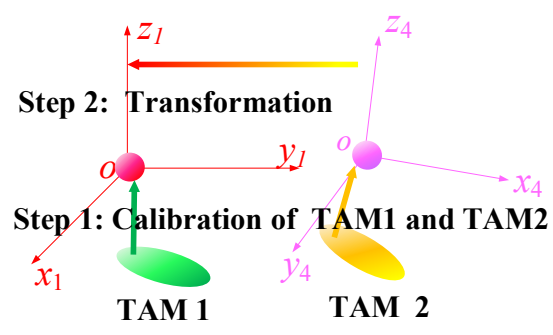

(a)

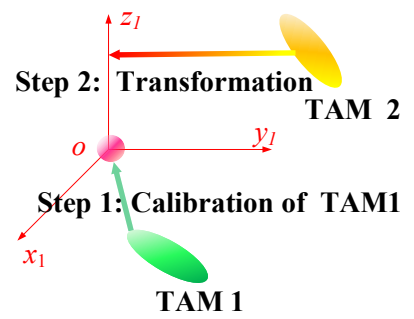

(b)

Figure 2.1: (a) Procedures of calibration methods in previous studies (b) Procedures of the calibration method proposed in this paper

In this paper, we propose a simplified calibration method to accelerate the calibration process. As shown in Fig 2.1, the new calibration method can also be divided into two steps. Unlike the existing calibration methods, only TAM1 is calibrated with the scalar calibration method in the first step, besides, self-errors of TAM2 and misalignment errors are calibrated as integral errors with vector calibration method in the second step. Without expensive magnetic field devices to provide reference magnetic field, the outputs of the calibrated TAM1 are considered as the reference magnetic field, in this way, self-errors of TAM2 and misalignment errors between two TAMs are calibrated as integral errors, calibrating self-errors of TAM2 is omitted and the calibration process gets simpler. In this study, we focus on the improvement of the calibration method of TAG without magnetic field devices. The paper is structured as follows: Section II includes calibration method of TAG; Section III includes the experiments and the results that verify the calibration method; conclusions are drawn in Section IV. 


\subsection{Calibration method of TAG}

\subsubsection{Calibration of TAM1}

Affected by sensitivity error, bias error and nonorthogonality error [71, 72], the outputs of TAM1 can be expressed as

$$
\boldsymbol{B}_{1}=\boldsymbol{A}_{1}\left(\boldsymbol{H}_{1}+\boldsymbol{b}_{1}+\boldsymbol{\zeta}_{1}\right)
$$

where $\boldsymbol{H}_{1}$ is the magnetic field to be estimated, $\boldsymbol{H}_{1}=\left[\begin{array}{lll}H_{x} & H_{y} & H_{z}\end{array}\right]^{T} . \boldsymbol{B}_{1}$ is the magnetic field measured by TAM1, $\boldsymbol{B}_{1}=\left[\begin{array}{lll}B_{x} & B_{y} & B_{z}\end{array}\right]^{T} . \boldsymbol{b}_{1}$ is the bias magnetic field, $\boldsymbol{b}_{1}=\left[\begin{array}{lll}b_{x} & b_{y} & b_{z}\end{array}\right]^{T} . \boldsymbol{\zeta}_{1}$ is the measurement noise, $\boldsymbol{\zeta}_{1}=$ $\left[\begin{array}{lll}\xi_{x} & \xi_{y} & \xi_{z}\end{array}\right]^{T} . A_{1}$ is the matrix with sensitivity error and nonorthogonality error, $\boldsymbol{A}_{1}^{-1}=\left[\begin{array}{lll}a_{11} & a_{12} & a_{13} \\ a_{12} & a_{22} & a_{23} \\ a_{13} & a_{23} & a_{33}\end{array}\right]$.

The parameter $\boldsymbol{A}, \boldsymbol{b}$ can be estimated by

$$
\left\|\boldsymbol{H}_{1}\right\|_{2}^{2}-H^{2}=\left\|\left(\boldsymbol{A}_{1}^{-1} \boldsymbol{B}_{1}-\boldsymbol{b}_{1}-\boldsymbol{\zeta}_{1}\right)\right\|-H^{2}=0
$$

where $\boldsymbol{H}_{1}$ is the estimated vector geomagnetic field, $H$ is the scalar geomagnetic field measured by the proton magnetometer, where the accuracy of proton magnetometer is $0.2 \mathrm{nT}$ that the measured value is reckoned as actual value. Eq. (2.3) can be obtained by expanding Eq. (2.2).

$$
\begin{aligned}
& k_{1} \boldsymbol{B}_{x}^{2}+k_{2} \boldsymbol{B}_{y}^{2}+k_{3} \boldsymbol{B}_{z}^{2}+k_{4} \boldsymbol{B}_{x} \boldsymbol{B}_{y}+k_{5} \boldsymbol{B}_{x} \boldsymbol{B}_{z}+k_{6} \boldsymbol{B}_{y} \boldsymbol{B}_{z}+ \\
& k_{7} \boldsymbol{B}_{x}+k_{8} \boldsymbol{B}_{y}+k_{9} \boldsymbol{B}_{z}=H^{2}+b_{x}^{2}+b_{y}^{2}+b_{z}^{2} \approx H^{2}
\end{aligned}
$$

where the noise magnetic field and bias magnetic field are relatively small compared with the geomagnetic field $H$, the noise magnetic field is not expanded in Eq. (2.3), at the same time, we get an approximation on the right side of Eq. (2.3). Eq. (2.3) represents a standard quadratic curve, the quadratic curve can be seen as an ellipsoid in this calibration situation 
[73]. Parameters of the ellipsoid can be expressed as

$$
\left\{\begin{array}{c}
k_{1}=a_{11}^{2}+a_{12}^{2}+a_{13}^{2} \\
k_{2}=a_{12}^{2}+a_{22}^{2}+a_{23}^{2} \\
k_{3}=a_{13}^{2}+a_{23}^{2}+a_{33}^{2} \\
k_{4}=2\left(a_{11} a_{12}+a_{12} a_{22}+a_{13} a_{23}\right) \\
k_{5}=2\left(a_{11} a_{13}+a_{12} a_{23}+a_{13} a_{33}\right) \\
k_{6}=2\left(a_{12} a_{13}+a_{22} a_{23}+a_{23} a_{33}\right) \\
k_{7}=-2\left(a_{11} b_{x}+a_{12} b_{y}+a_{13} b_{z}\right) \\
k_{8}=-2\left(a_{12} b_{x}+a_{22} b_{y}+a_{23} b_{z}\right) \\
k_{9}=-2\left(a_{13} b_{x}+a_{23} b_{y}+a_{33} b_{z}\right)
\end{array}\right.
$$

When there are $\mathrm{N}$ sets of measured data, $\mathrm{N}$ sets of equations can be gotten from Eq. (2.3).

$$
\boldsymbol{B}_{M} \boldsymbol{K}=\boldsymbol{b}_{M}
$$

where

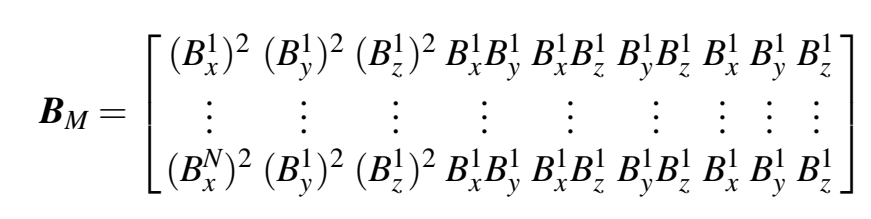

$\boldsymbol{B}_{x}^{i}, \boldsymbol{B}_{y}^{i}, \boldsymbol{B}_{z}^{i}(i=1 \cdots \mathrm{N})$ are the Nth set outputs of TAM1.

$$
\begin{gathered}
\boldsymbol{K}=\left[\begin{array}{lllllllll}
k_{1} & k_{2} & k_{3} & k_{4} & k_{5} & k_{6} & k_{7} & k_{8} & k_{9}
\end{array}\right] \\
\boldsymbol{b}_{M}=\left[\begin{array}{lllllllll}
H^{2} & H^{2} & H^{2} & H^{2} & H^{2} & H^{2} & H^{2} & H^{2} & H^{2}
\end{array}\right]
\end{gathered}
$$

When $\mathrm{N} \geq 9$ and the measured matrix $\boldsymbol{B}_{M}$ is reversible, a unique vector $\boldsymbol{K}$ can be gotten.

$$
\boldsymbol{K}=\left(\left(\boldsymbol{B}_{M}\right)^{T} \boldsymbol{B}_{M}\right)^{-1}\left(\boldsymbol{B}_{M}\right)^{T} \boldsymbol{b}_{M}
$$

After $\boldsymbol{K}$ is estimated, bring $\boldsymbol{K}$ to Eqs. (4), the parameter $\boldsymbol{A}_{1}, \boldsymbol{b}_{1}$ can be estimated by solving the nonlinear equations, the initial value of $\boldsymbol{A}_{1}, \boldsymbol{b}_{1}$ can be selected as the parameters of ideal TAM without errors.

$$
\left(\boldsymbol{A}_{1}^{-1}\right)_{\text {initial }}=\left[\begin{array}{lll}
1 & 0 & 0 \\
0 & 1 & 0 \\
0 & 0 & 1
\end{array}\right],\left(\boldsymbol{b}_{1}\right)_{\text {initial }}=\left[\begin{array}{lll}
0 & 0 & 0
\end{array}\right]
$$




\subsubsection{Vector calibration of TAM2}

Similar to TAM1, the outputs of TAM2 can be expressed as

$$
\boldsymbol{B}_{2}=\boldsymbol{A}_{2}\left(\boldsymbol{H}_{2}+\boldsymbol{b}_{2}+\boldsymbol{\zeta}_{2}\right)
$$

where $\boldsymbol{H}_{2}$ is the magnetic field to be estimated of TAM2, $\boldsymbol{B}_{2}$ is the bias magnetic field, $\boldsymbol{\zeta}_{2}$ is the measured noise, $\boldsymbol{A}_{2}$ is the error matrix including the sensitivity error and nonorthogonality error. When the measurement noise $\zeta_{1}, \zeta_{2}$ are neglected, according to Eq. (2.1) and Eq. (2.11), the estimated magnetic field can be expressed as

$$
\left\{\begin{array}{l}
\boldsymbol{H}_{1}=\boldsymbol{A}_{1}^{-1} \boldsymbol{B}_{1}-\boldsymbol{b}_{1} \\
\boldsymbol{H}_{2}=\boldsymbol{A}_{2}^{-1} \boldsymbol{B}_{2}-\boldsymbol{b}_{2}
\end{array}\right.
$$

After calibration, the estimated values of $\boldsymbol{H}_{1}$ and $\boldsymbol{H}_{2}$ may be located in two coordinate systems, as shown in Fig 2.2. $\boldsymbol{H}_{2}$ can be transformed to the coor-

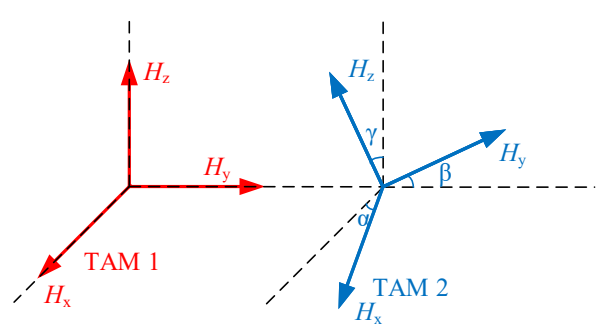

Figure 2.2: Relative relations between calibrated data of TAM1 and TAM2

dinate system of TAM1 through a transform matrix.

$$
\overline{\boldsymbol{H}}_{2}=\boldsymbol{C H}_{2}
$$

where $\boldsymbol{C}$ is the transition matrix between two orthogonal coordinate systems, $\overline{\boldsymbol{H}}_{2}$ is the transformed value of $\boldsymbol{H}_{2}, \overline{\boldsymbol{H}}_{2}$ and $\boldsymbol{H}_{1}$ are in the same coordinate system. According to the homogeneity of the measured magnetic field, $\overline{\boldsymbol{H}}_{2}$ equals to $\boldsymbol{H}_{1}$.

$$
\boldsymbol{H}_{1}-\boldsymbol{C}\left(\boldsymbol{A}_{2}^{-1} \boldsymbol{B}_{2}-\boldsymbol{b}_{2}\right)=0
$$

Eq. (2.14) can be transformed

$$
\left[\begin{array}{ll}
\overline{\boldsymbol{A}}_{2} & \overline{\boldsymbol{b}}_{2}
\end{array}\right]\left[\begin{array}{l}
\boldsymbol{B}_{2} \\
-1
\end{array}\right]=\overline{\boldsymbol{H}}_{1}
$$


where $\overline{\boldsymbol{H}}_{1}$ is the estimated value of $\boldsymbol{H}_{1}$ from subsection A. Since $\boldsymbol{H}_{1}$ is the magnetic field to be estimated, $\boldsymbol{H}_{1}$ can be obtained through reference magnetic field in traditional vector calibration method, the magnetic field devices and the orientation instruments are so precise that the measured value of $\boldsymbol{H}_{1}$ can be considered as the true value. In this paper, the estimated $\overline{\boldsymbol{H}}_{1}$ is used to replace the true value of $\boldsymbol{H}_{1}$, when there are $\mathrm{N}$ sets of measured points, a set of equations can be obtained.

$$
\left[\begin{array}{ll}
\overline{\boldsymbol{A}}_{2} & \overline{\boldsymbol{b}}_{2}
\end{array}\right]\left[\begin{array}{ccc}
\boldsymbol{B}_{2}^{1} & \cdots & \boldsymbol{B}_{2}^{N} \\
-1 & -1 & -1
\end{array}\right]=\overline{\boldsymbol{H}}_{1}^{1} \quad \cdots \quad \overline{\boldsymbol{H}}_{1}^{N}
$$

Eq. (2.16) can be simplified as

$$
\left[\begin{array}{ll}
\bar{A}_{2} & \bar{b}_{2}
\end{array}\right] \boldsymbol{B}=\boldsymbol{H}
$$

where

$$
\boldsymbol{B}=\left[\begin{array}{lll}
\boldsymbol{B}_{2}^{1} & \cdots & \boldsymbol{B}_{2}^{N} \\
-1 & -1 & -1
\end{array}\right]_{4 \times N}, \boldsymbol{H}=\left[\begin{array}{lll}
\bar{H}_{1}^{1} & \ldots & \bar{H}_{1}^{N}
\end{array}\right]_{3 \times N}
$$

When $\mathrm{N} \geq 3$ and all the measured points are not on the same plane, the parameters can be solved uniquely which can be expressed as

$$
\left.\left[\begin{array}{ll}
\bar{A}_{2} & \overline{\boldsymbol{b}}_{2}
\end{array}\right]=\boldsymbol{H} \boldsymbol{B}^{T}(\boldsymbol{B B})^{T}\right)^{-1}
$$

After $\overline{\boldsymbol{A}}_{2}, \overline{\boldsymbol{b}}_{2}$ are estimated, the measured data of TAM2 can be calibrated in the coordinate system of TAM1.

\subsection{Experiments}

\subsubsection{Experiment setups}

The experiment was performed in the suburbs of Xi' an area, which is far from the industrial facilities and has stable magnetic environment. The experimental setups include a GSM-19T proton magnetometer, a digital TAG, a two-axis nonmagnetic turntable, a notebook computer, a portable power supply and a pipeline. The proton magnetometer is used to get the scalar geomagnetic field. The digital TAG is provided by Xi' an Hua Shun company, the resolution is better than $1 \mathrm{nT}$. The two-axis nonmagnetic turntable is mainly composed of wood and engineering plastics, the smallest scale 
that can be read is 0.1 degree, the turntable is assisted to get steady outputs in space, the angle information is not used in the calibration procedure. The notebook computer is equipped with LabVIEW program, mainly used to read the TAG and process the data. The portable $12 \mathrm{~V}$ power supply is used to supply the TAG. The pipeline is used to testify the performance of the calibrated TAG, the length of the pipeline is $110 \mathrm{~cm}$, the inside and outside diameters are $4 \mathrm{~cm}$ and $8 \mathrm{~cm}$ respectively. Two experiments were performed. Firstly, the magnetometer was rotated in 3D space with the aid of two-axis turntable. Secondly, the calibration performance was verified by detecting oil tube.

\subsubsection{Experiment of calibration}

Set up the experimental devices, power up for 30 minutes to warm up the system. Estimation of parameters in the calibration method require enough data which are widely distributed in the space. In this experiment, we use the two-axis turntable to rotate the TAG three times along $\mathrm{x}$-axis, $\mathrm{y}$-axis, $\mathrm{z}$-axis respectively to get enough measuring points in space. During the rotation process, stop $10 \mathrm{~s}$ for every 30 degrees, then the data in the static $10 \mathrm{~s}$ will be the averaged. With this method, the random noise can be reduced, while the time is relatively short, the error caused by the drift of geomagnetic field can be ignored. Rotate the TAG about three axes respectively, after the rotation process, three sets of data from different rotation axis can be obtained, bring the data to the model, parameters of the TAG can be estimated, as shown in Table I. The corresponding results are shown in Fig 2.3.

Table 2.1: Estimated Parameters With The Proposed Calibration Method

\begin{tabular}{cc}
\hline Parameters & Estimated Values \\
\hline $\boldsymbol{A}_{1}$ & {$\left[\begin{array}{ccc}0.9990 & -0.0013 & -0.0202 \\
-0.0013 & 1.0049 & -0.0006 \\
-0.0202 & -0.0006 & 1.0039\end{array}\right]$} \\
$\boldsymbol{b}_{1}$ & {$\left[\begin{array}{ccc}81.5 & 71.5 & 29.3\end{array}\right]^{T}$} \\
$\boldsymbol{A}_{2}$ & {$\left[\begin{array}{ccc}0.9995 & 0.0024 & -0.0380 \\
-0.0049 & 1.0070 & 0.0025 \\
-0.0023 & -0.0039 & 1.0064\end{array}\right]$} \\
$\boldsymbol{b}_{2}$ & {$\left[\begin{array}{lll}23.0 & 0.8 & -5.5\end{array}\right]^{T}$} \\
\hline
\end{tabular}


2 Hybrid Calibration Method for Three-axis Gradiometer

Table 2.2: Calibration Errors with Traditional Calibration Method and Proposed One

\begin{tabular}{lcccc}
\hline & & X-axis(nT) & Y-axis(nT) & Z-axis(nT) \\
\hline \multirow{4}{*}{ The tradition method } & Max & 95.4 & 89.0 & 90.3 \\
& Mean & -10.3 & 6.8 & 1.3 \\
& Std & 31.5 & 22.8 & 21.0 \\
The Proposed method & Max & 94.2 & 71.7 & 87.1 \\
& Mean & 0.05 & 0.02 & 0.07 \\
& Std & 24.5 & 18.7 & 20.9 \\
\hline
\end{tabular}

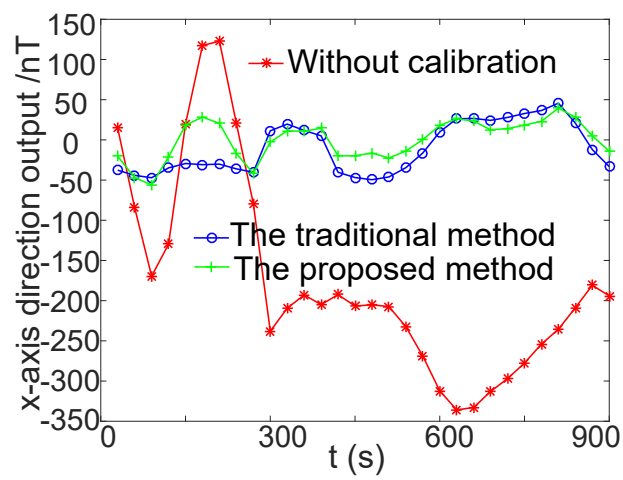

(a)

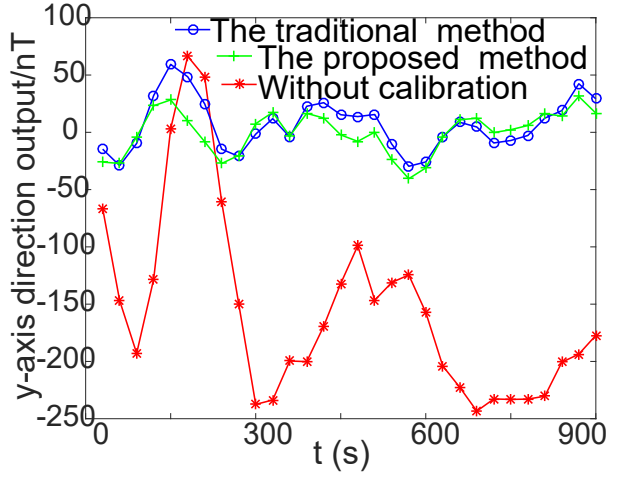

(b)

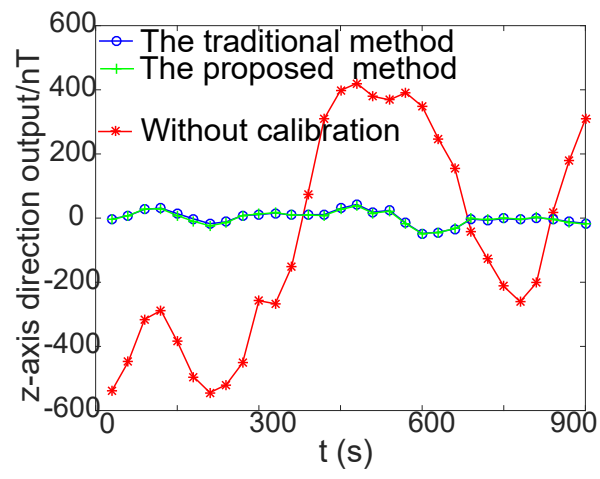

(c)

Figure 2.3: (a) Scalar output of TAM1 (b) Scalar output of TAM2 (c) Vector outputs along $x$-axis direction 


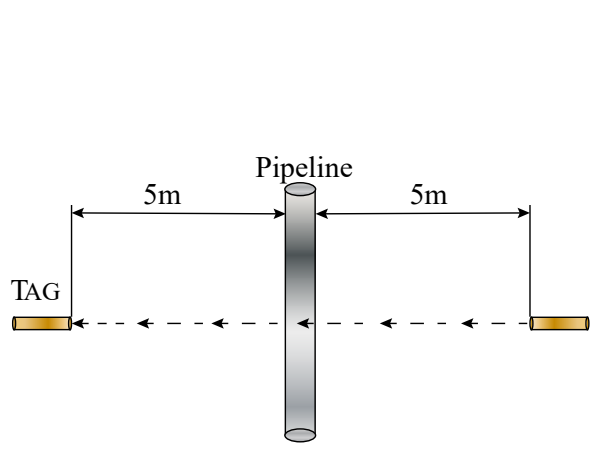

(a)

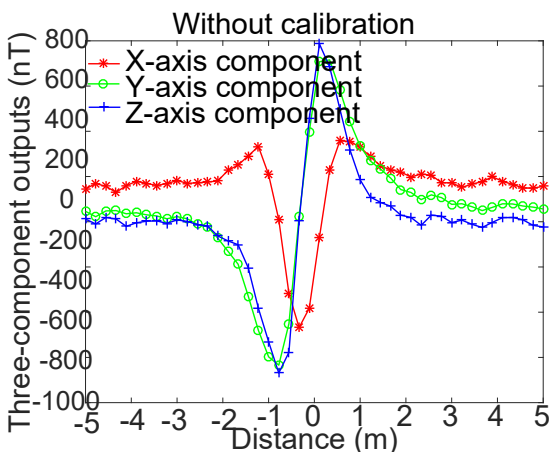

(b)

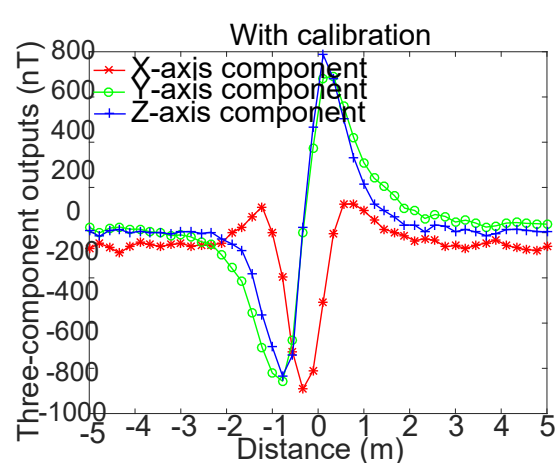

(c)

Figure 2.4: (a) Detecting path of the pipline in the experiment (b) Outputs of TAG without calibration (c) Outputs of TAG with calibration 
As shown in Fig 2.3, the vector output errors were reduced from 383.6 $\mathrm{nT}, 306.1 \mathrm{nT}, 970.8 \mathrm{nT}$ to $94.2 \mathrm{nT}, 71.7 \mathrm{nT}, 87.1 \mathrm{nT}$ respectively with the proposed calibration method. As shown in TABLE II, Max represents the maximum error (Peak to Peak) along three axes, Mean represents the mean value of the outputs along axes, Std represents standard deviation of the TAG outputs. Compared with the traditional calibration method which is from [69], the mean values of the outputs were improved enormously while the maximum errors and the standard deviation of the TAG outputs were improved slightly. The traditional calibration algorithm took $1552.64 \mathrm{~ms}$ while the proposed method took $779.28 \mathrm{~ms}$, the program was running in Matlab 2016 (64 bit) in Win 10 operating system. The performance of the calibration method is not ideal, the errors were caused by many factors such as external magnetic distortions, the non-linearity of the TAG outputs or the temperature drifts. The mean value of the outputs were improved tremendously compared with the traditional method because it had only one reference while the traditional method has two references, the unexpected errors can cause larger deviations between two references.

\subsubsection{Detection of Oil Tube}

After the calibration procedure, the second experiment was conducted to testify the detecting capability of the TAG, we chosen a pipeline as a detection object. Firstly, put the pipeline on the ground. Secondly, approach the pipeline with the TAG in the hand, the TAG is about $1.2 \mathrm{~m}$ above the pipeline with a fixed direction, the moving direction was shown as Fig 2.4a.

As shown in Fig 2.4b and 2.4c, the outputs of TAG had relatively high deviation errors before calibration when the TAG was far from the pipeline, after the calibration, the deviation errors became smaller. The advantage of the calibration is that it will be easier to estimate whether there is a ferromagnetic object when the outputs are relatively small.

\subsection{Discussion}

Calibration is one of the crucial factors for the application of TAGs. In this paper, a hybrid calibration method was proposed to calibrate gradiometer or magnetometer array including more than two magnetometers. For the previous studies, TAM1 and TAM2 were calibrated individually based on scalar 
calibration method, then the misalignment errors between two magnetometers were estimated. In this paper, self-errors of TAM1 are calibrated based on the scalar calibration method in first step, self-errors of TAM 2 and misalignment errors were calibrated by vector calibration method in second step. The advantage of the proposed calibration method is self-errors of TAM2 and the misalignment errors are calibrated in one step with linear method, the calibration process is simplified with less computations. This method is also suitable for other systems with more TAMs, such as the tensor system.

As at least 9 independent data points can determine an ellipsoid, the calibration data points must larger than 9. In our experiments, we exploited 30 averaged points, which were enough for the calibration.

\subsection{Conclusion}

A complete calibration method for TAG is proposed in this paper. Two experiments were conducted. Firstly, the TAG was calibrated in a stable geomagnetic environment, the experiment results showed that the scalar and vector output errors were greatly reduced, then the performance of calibrated TAG was testified by detecting the pipeline on the ground, the outputs of TAG were reduced when there is no ferromagnetic object, it was easier to determine whether there was a detecting object when the outputs were relatively small. 
CHAPTER 3

\section{Calibration of tri-axis magnetometers based on improved truncated singular value decomposition method}

This chapter is published as a journal article: Zhicheng, Y., Bin, L., \& Lianping, C. (2018). Calibration of tri-axis magnetometers using an improved truncated singular value decomposition method. Measurement Science and Technology, 29(12), 125101. 


\title{
Calibration of tri-axis magnetometers using an improved truncated singular value decomposition method
}

\begin{abstract}
The performance of three-axis magnetometer (TAM) is limited by different kinds of errors, calibration must be performed prior its use. The convergences of calibration algorithms are quite sensitive to input data, divergent issues would cause uncorrected parameter estimation results when the maneuvers of TAM are constrained. In light of this situation, a calibration method is proposed which is primarily based on improved truncated singular value decomposition. The singular values are divided into groups, the smaller singular values are modified while the bigger ones stay the same. In this way, the more accurate information with bigger singular values remains the same while the less accurate information contained in smaller singular values is considered and modified to avoid the divergent problem. the experimental results show, the performance of TAM is improved dramatically with the proposed calibration method.
\end{abstract}




\subsection{Introduction}

Three-axis magnetometer(TAM)s are widely used in navigation, unexploded ordnance(UXO) detection, geological survey [74, 75, 76].The performance of TAM is corrupted by sensitivity error, offset error, nonorthogonality error, soft effect and hard effect disturbance. Affected by these errors, outputs of TAM are deviated from the true values within thousands of nanoteslas, which is unacceptable for many measuring occasions. Thus, TAM needs to be calibrated in order to compensate for these undesired effects. A large amount of work regarding TAM calibration algorithms has been done in recent years. Merayo et al introduced a scalar calibration method which only needs a reference scalar magnetometer, the advantage of this method lies in its linear least square estimator, it can estimate unique parameters for a set of given data without iterations [77]. Alonso introduced a two-step method which first estimates a coarse value using the centering operation and then applies a least square solver to obtain fine estimation [78]. C. C. FOSTER did some research by considering nonorthogonal error to the nonlinear two-step estimation model [79]. Auster et al introduced a method by using relative motions, no coil systems or precise mechanics are needed [80]. Some methods for the estimation of error parameters are proposed to improve the performance of Meray's and Alonso's method, D. Gebre-Egziabher et al proposed a non-linear two step estimator for the parameters estimation where the first step problem is solved by using standard batch least squares linear estimation techniques and the second step is solved algebraically [81, 82]. Pylvanainen proposed a recursive fitting method, the advantage of this method lies in that parameters can converge from any starting point and update adaptively [83]. Valérie proposed an adaptive least squares estimator which provides a consistent solution and converges to a good estimate of the said errors, the heading accuracy improved tremendously after calibration [84]. Pan proposed a new error model by considering nonlinearity coefficients of scale factors to improve the calibration performance [85], he also introduced the nonlinear method and integrated method for a better estimation of the error parameter [86, 87]. Yang et al introduced a hybrid calibration method for tri-axis gradiometers, which is suitable for the calibration of the system with two or more TAMs [88].

The premise of calibration methods above is that measuring points distribute on the ellipsoid uniformly so that algorithms can converge. Alberto introduced a method to monitor and assess the goodness of the spatial dis- 
tribution of the gathered data on the ellipsoid [89], it can give feedback to the operator when the dataset is ready. There are few methods corresponding to the situation when the operation of TAM is restricted. Wu introduced a truncated total least squares (TTLS) technique to estimate the error parameters, which can achieve good calibration performance in the restricted situation [90]. But in reference [90], only the bigger singular values are utilized which would degrade the calibration performance. In this paper, an improved truncated singular value decomposition method is proposed. Both the bigger and smaller singular values are used, the bigger singular values are processed with the truncated singular value decomposition method, the smaller singular values are modified instead of being discard directly, not only the divergent problem is solved, but also the calibration accuracy is improved.

\subsection{Error model of the TAM}

The outputs of TAM can be expressed as

$$
B=S_{1} S_{2} \boldsymbol{N}(H+c+h+\xi)
$$

where $\boldsymbol{B}$ is magnetic field measured from the TAM, $\boldsymbol{B}=\left[\begin{array}{lll}B_{x} & B_{y} & B_{z}\end{array}\right]^{T}$. $S_{1}$ is the soft interference coefficient. $S_{2}$ is the sensitivity error coefficient. $\boldsymbol{N}$ is the nonorthogonality error coefficient. $\boldsymbol{H}$ denotes magnetic field to be estimated, $\boldsymbol{H}=\left[\begin{array}{lll}H_{x} & H_{y} & H_{z}\end{array}\right]^{T}$. $\boldsymbol{c}$ is the bias error. $\boldsymbol{h}$ denotes the hard interference coefficient. $\boldsymbol{\xi}$ is the measuring noise of TAM, $\boldsymbol{\xi}=\left[\begin{array}{lll}\xi_{x} & \xi_{y} & \xi_{z}\end{array}\right]^{T}$. These parameters can be estimated as integrated error parameters.

$$
\boldsymbol{B}=\boldsymbol{A}(\boldsymbol{H}+\boldsymbol{b}+\boldsymbol{\xi})
$$

where $\boldsymbol{A}$ is the integrated soft interference parameter, $\boldsymbol{A}^{-1}=\left(\boldsymbol{S}_{1} \boldsymbol{S}_{2} \boldsymbol{N}\right)^{-1}=$ $\left[\begin{array}{lll}a_{11} & a_{11} & a_{11} \\ a_{21} & a_{22} & a_{23} \\ a_{31} & a_{32} & a_{33}\end{array}\right], \boldsymbol{b}$ is integrated hard interference parameter, $\boldsymbol{b}=\boldsymbol{c}+\boldsymbol{h}=$
$\left[\begin{array}{lll}b_{x} & b_{y} & b_{z}\end{array}\right]$. The parameter $\boldsymbol{A}, \boldsymbol{b}$ can be estimated by

$$
\left\|\boldsymbol{H}_{1}\right\|_{2}^{2}-H^{2}=\left\|\boldsymbol{A}^{-1} \boldsymbol{B}-\boldsymbol{b}-\boldsymbol{\xi}\right\|_{2}^{2}-H^{2}=0
$$

where $\boldsymbol{H}_{1}$ is the estimated vector geomagnetic field, $H$ is the local geomagnetic field. Eq (3.4) can be obtained by expanding Eq (3.3)

$k_{1} \boldsymbol{B}_{x}^{2}+k_{2} \boldsymbol{B}_{y}^{2}+k_{3} \boldsymbol{B}_{z}^{2}+2 k_{4} \boldsymbol{B}_{x} \boldsymbol{B}_{y}+2 k_{5} \boldsymbol{B}_{x} \boldsymbol{B}_{z}+2 k_{6} B_{y} \boldsymbol{B}_{z}+k_{7} \boldsymbol{B}_{x}+k_{8} \boldsymbol{B}_{y}+k_{9} \boldsymbol{B}_{z}=1$ 
where the noise magnetic field is relatively small compared with the magnetic field $H$ and is not expanded in Eq (3.4). Eq (3.4) represents a standard quadratic curve, the quadratic curve can be seen as an ellipsoid in this calibration situation [91]. Parameters of the ellipsoid can be expressed as

$$
\left\{\begin{array}{l}
k_{1}=\left(a_{11}^{2}+a_{12}^{2}+a_{13}^{2}\right) / T \\
k_{2}=\left(a_{12}^{2}+a_{22}^{2}+a_{23}^{2}\right) / T \\
k_{3}=\left(a_{13}^{2}+a_{23}^{2}+a_{33}^{2}\right) / T \\
k_{4}=2\left(a_{11} a_{12}+a_{12} a_{22}+a_{13} a_{23}\right) / T \\
k_{5}=2\left(a_{11} a_{13}+a_{12} a_{23}+a_{13} a_{33}\right) / T \\
k_{6}=2\left(a_{12} a_{13}+a_{22} a_{23}+a_{23} a_{33}\right) / T \\
k_{7}=-2\left(a_{11} b_{x}+a_{12} b_{y}+a_{13} b_{z}\right) / T \\
k_{8}=-2\left(a_{12} b_{x}+a_{22} b_{y}+a_{23} b_{z}\right) / T \\
k_{9}=-2\left(a_{13} b_{x}+a_{23} b_{y}+a_{33} b_{z}\right) / T \\
T=H^{2}+b_{x}^{2}+b_{y}^{2}+b_{z}^{2}
\end{array}\right.
$$

where $T$ is a scalar factor. When there are $N$ sets of measured data, $N$ sets of equations can be gotten from $\mathrm{Eq}$ (3.4)

$$
\boldsymbol{B}_{M} \boldsymbol{K}=\boldsymbol{b}_{M}
$$

where

$$
\begin{gathered}
\boldsymbol{B}_{\boldsymbol{M}}=\left[\begin{array}{ccccccccc}
\left(B_{x}^{1}\right)^{2} & \left(B_{y}^{1}\right)^{2} & \left(B_{z}^{1}\right)^{2} & B_{x}^{1} B_{y}^{1} & B_{x}^{1} B_{z}^{1} & B_{y}^{1} B_{z}^{1} & B_{x}^{1} & B_{y}^{1} & B_{z}^{1} \\
\vdots & \vdots & \vdots & \vdots & \vdots & \vdots & \vdots & \vdots & \vdots \\
\left(B_{x}^{N}\right)^{2} & \left(B_{y}^{N}\right)^{2} & \left(B_{z}^{N}\right)^{2} & B_{x}^{N} B_{y}^{N} & B_{x}^{N} B_{z}^{N} & B_{y}^{N} B_{z}^{N} & B_{x}^{N} & B_{y}^{N} & B_{z}^{N}
\end{array}\right] \\
\boldsymbol{K}=\left[\begin{array}{ccccccccc}
k_{1} & k_{2} & k_{3} & k_{4} & k_{5} & k_{6} & k_{7} & k_{8} & k_{9}
\end{array}\right]^{T} \\
\boldsymbol{b}_{M}=\left[\begin{array}{cccc}
1 & \cdots & 1
\end{array}\right]_{N}^{T}
\end{gathered}
$$

$B_{x}^{i}, B_{y}^{i}, B_{z}^{i}(i=1 \cdots N)$ represent the $N$ th set outputs of TAM. The measuring data set $N$ usually much bigger than 9 , so there is no exact solution for $\boldsymbol{K}$ in $\mathrm{Eq}$ (3.6). But the unique least-squares solution $\boldsymbol{K}$ can be gotten when matrix $\boldsymbol{B}_{\boldsymbol{M}}$ has a full column rank.

$$
\boldsymbol{K}=\left(\left(\boldsymbol{B}_{\boldsymbol{M}}\right)^{T} \boldsymbol{B}_{\boldsymbol{M}}\right)^{-1}\left(\boldsymbol{B}_{\boldsymbol{M}}\right)^{T} \boldsymbol{b}_{\boldsymbol{M}}
$$

After $\boldsymbol{K}$ is estimated, bring $\boldsymbol{K}$ to $\mathrm{Eq}$ (3.5), the parameter $\boldsymbol{A}, \boldsymbol{b}$ can be estimated by solving nonlinear equations. But the full column rank of $\boldsymbol{B}_{\boldsymbol{M}}$ 
requires enough measuring points widely distribute on the ellipsoid. When the movement of the TAM is restricted, there are strong correlations among the measuring points, therefore, the matrix $\boldsymbol{B}_{\boldsymbol{M}}$ in Eq (3.7) is rank deficient which means Eq (3.6) is ill-conditioned, the estimation of $\boldsymbol{K}$ will have a much higher deviation when we use direct inverse of $\left(\boldsymbol{B}_{\boldsymbol{M}}\right)^{T} \boldsymbol{B}_{\boldsymbol{M}}$.

\subsection{Regularized calibration method}

In the error model of TAM, The local geomagnetic field $H$ is regarded as constant. Thus, $\boldsymbol{b}_{M}$ can be expressed Eq (3.9). However, The $H$ in Eq (3.5) changes slowly, besides, there are measurement noises for the geomagnetic field. $\boldsymbol{b}_{M}$ can be expressed as

$$
\boldsymbol{b}_{M}=\boldsymbol{b}_{\text {true }}+\boldsymbol{b}_{\text {noise }}
$$

where $\boldsymbol{b}_{\text {true }}=\left[\begin{array}{ccc}1 & \cdots & 1\end{array}\right]_{N}^{T}, \boldsymbol{b}_{\text {noise }}$ is related to measurement noise and slow variation of geomagnetic field.

Let $\boldsymbol{B}_{M}^{+}$denotes the Moore-penrose pseudoinverse of $\boldsymbol{B}_{M}$, parameter $\boldsymbol{K}$ is determined as an approximate solution of the least-squares problem. Note that the solution can be expressed as

$$
\widetilde{\boldsymbol{K}}=\boldsymbol{B}_{M}^{+} \boldsymbol{b}_{M}=\boldsymbol{B}_{M}^{+}\left(\boldsymbol{b}_{\text {true }}+\boldsymbol{b}_{\text {noise }}\right)
$$

The singular value decomposition of $\boldsymbol{B}_{\boldsymbol{M}}$ in Eq (3.6) can be expressed as

$$
\boldsymbol{B}_{\boldsymbol{M}}=\boldsymbol{U} \boldsymbol{\Sigma} \boldsymbol{V}^{T}=\sum_{i=1}^{9} \sigma_{i} \boldsymbol{u}_{i} \boldsymbol{v}_{i}^{T}
$$

where the left and right singular matrix $\boldsymbol{U}^{N \times 9}$ and $\boldsymbol{V}^{9 \times 9}$ are orthogonal, $\boldsymbol{U}=$ $\left[\begin{array}{lll}\boldsymbol{u}_{1} & \cdots & \boldsymbol{u}_{9}\end{array}\right], \boldsymbol{V}=\left[\begin{array}{lll}\boldsymbol{v}_{1} & \cdots & \boldsymbol{v}_{9}\end{array}\right], \boldsymbol{\Sigma}=\operatorname{diag}\left(\sigma_{1}, \cdots, \sigma_{9}\right), \sigma_{1} \geq \sigma_{2} \geq \cdots \geq$ $\sigma_{9}$, elements $\sigma_{i}$ is the $i$ th singular value of $\boldsymbol{B}_{\boldsymbol{M}}$. With the SVD technique, the estimation in $\mathrm{Eq}(3.10)$ can be equally expressed as

$$
\widetilde{\boldsymbol{K}}=\sum_{i=1}^{9} \frac{\left(\boldsymbol{u}_{i}^{T} \boldsymbol{b}_{\text {true }}\right) \boldsymbol{v}_{i}}{\sigma_{i}}+\sum_{i=1}^{k} \frac{\left(\boldsymbol{u}_{i}^{T} \boldsymbol{b}_{\text {noise }}\right) \boldsymbol{v}_{i}}{\sigma_{i}}+\sum_{i=k+1}^{9} \frac{\left(\boldsymbol{u}_{i}^{T} \boldsymbol{b}_{\text {noise }}\right) \boldsymbol{v}_{i}}{\sigma_{i}}
$$

When $\boldsymbol{B}_{\boldsymbol{M}}$ is rank deficient, the last few singular values $\sigma_{i}$ are close to zero, $1 / \sigma_{i}$ will introduce a large deviation. Typically, $\widetilde{\boldsymbol{K}}$ is dominated by the third 
part in Eq (3.14) which is known as propagated error and then is meaningless. That is the reason why the estimation of $\boldsymbol{K}$ is far from the true value. To solve this problem, the Tikhonov regulation method and truncated singular value decomposition (TSVD) method are two most widely used methods.

\subsubsection{Tikhonov method}

As to the Tikhonov method, it sets a new cost function to replace the old one [92]

$$
\widetilde{\boldsymbol{K}}_{\text {Tikhonov }}=\underset{K}{\operatorname{argmin}}\left\{\left\|\boldsymbol{B}_{\boldsymbol{M}} \boldsymbol{K}-\boldsymbol{b}_{\boldsymbol{M}}\right\|_{2}^{2}+\lambda^{2}\left\|\boldsymbol{L}\left(\boldsymbol{K}-\boldsymbol{K}_{0}\right)\right\|_{2}^{2}\right\}
$$

where the additional item $\left\|\boldsymbol{L}\left(\boldsymbol{K}-\boldsymbol{K}_{0}\right)\right\|_{2}^{2}$ is used to constrain the square of residual norm, it can be used to measure the performance of the estimation of $\boldsymbol{K}$. When the matrix $\boldsymbol{L}$ is set as unit matrix and $\boldsymbol{K}_{0}$ is set as zero matrix, Eq (3.15) can be simplified as standard Tikhonov form

$$
\widetilde{\boldsymbol{K}}_{\text {Tikhonov }}=\underset{K}{\operatorname{argmin}}\left\{\left\|\boldsymbol{B}_{\boldsymbol{M}} \boldsymbol{K}-\boldsymbol{b}_{\boldsymbol{M}}\right\|_{2}^{2}+\lambda^{2}\|\boldsymbol{K}\|_{2}^{2}\right\}
$$

Eq (3.16) can be converted to

$$
\left(\boldsymbol{B}_{M}^{T} \boldsymbol{B}_{M}+\lambda^{2} \boldsymbol{I}_{9}\right) \boldsymbol{K}=\boldsymbol{B}_{\boldsymbol{M}}^{T} \boldsymbol{b}_{\boldsymbol{M}}
$$

Then the estimation of $\boldsymbol{K}$ can be expressed as

$$
\begin{aligned}
\widetilde{\boldsymbol{K}}_{\text {Tikhonov }} & =\left(\boldsymbol{B}_{\boldsymbol{M}}^{T} \boldsymbol{B}_{\boldsymbol{M}}+\lambda^{2} \boldsymbol{I}_{9}\right)^{-1} \boldsymbol{B}_{\boldsymbol{M}}^{T} \boldsymbol{b}_{\boldsymbol{M}}=\sum_{i=1}^{9} \frac{\sigma_{i}^{2}}{\sigma_{i}^{2}+\lambda^{2}} \frac{\left(\boldsymbol{u}_{i}^{T} \boldsymbol{b}_{\boldsymbol{M}}\right) \boldsymbol{v}_{i}}{\sigma_{i}} \\
& =\sum_{i=1}^{9} f_{i}\left(\boldsymbol{u}_{i}^{T} \boldsymbol{b}_{\boldsymbol{M}}\right) \boldsymbol{v}_{i}
\end{aligned}
$$

$f_{i}$ can be denoted as

$$
f_{i}=\frac{\sigma_{i}}{\sigma_{i}^{2}+\lambda^{2}}
$$

where $\lambda$ is set between two singular values $\sigma_{k+1} \leq \lambda \leq \sigma_{k}$. When $i>k$, the singular value $\sigma_{i}$ approaches zero very fast, the item $\sigma_{i}^{2} /\left(\sigma_{i}^{2}+\lambda^{2}\right)$ can become very small correspondingly. Thus, the error introduced by small singular values is damped. 
3 Calibration of magnetometers based on improved SVD method

\subsubsection{TSVD method}

When it comes to the TSVD method, the basic idea is to replace the illconditioned matrix $\boldsymbol{B}_{\boldsymbol{M}}$ with a well-conditioned matrix $\boldsymbol{B}_{T S V D}$, where $\boldsymbol{B}_{T S V D}$ can be expressed as

$$
\boldsymbol{B}_{T S V D}=\sum_{i=1}^{k} \sigma_{i} u_{i} v_{i}^{T}
$$

When $i>k$, the singular values are set as zeroes. The regularized estimation of $\boldsymbol{K}$ can be expressed as

$$
\widetilde{\boldsymbol{K}}_{T S V D}=\boldsymbol{B}_{T S V D}^{+} \boldsymbol{b}_{m}
$$

where $\boldsymbol{B}_{T S V D}^{+}$is the pseudoinverse of $\boldsymbol{B}_{T S V D}, \boldsymbol{B}_{T S V D}^{+}=\boldsymbol{V} \boldsymbol{\Sigma}_{T S V D}^{+} \boldsymbol{U}^{T}, \boldsymbol{\Sigma}_{T S V D}^{+}=$ $\left(\sigma_{1}^{-1}, \cdots, \sigma_{k}^{-1}, \cdots, 0\right)$, it can be also expressed as

$$
\widetilde{\boldsymbol{K}}_{T S V D}=\sum_{i=1}^{k} \frac{\left(\boldsymbol{u}_{i}^{T} \boldsymbol{b}_{\boldsymbol{M}}\right) \boldsymbol{v}_{i}}{\sigma_{i}}=\sum_{i=1}^{9} g_{i}\left(\boldsymbol{u}_{i}^{T} \boldsymbol{b}_{\boldsymbol{M}}\right) \boldsymbol{v}_{i}
$$

where $g_{i}=\left\{\begin{array}{cc}1 / \sigma_{i} & 1 \leq i \leq k \\ 0 & k<i \leq 9\end{array}\right.$. Compared with the Eq (3.14), the propagated errors introduced by last few singular values are eliminated. The estimation of $\boldsymbol{K}$ becomes more accurate.

\subsubsection{Improved TSVD method}

For TSVD method, it can be seen the singular problem is solved by neglecting the smaller singular values, the solution can be very robust but the estimation of $\boldsymbol{K}$ is also affected since the information related to the smaller singular values is neglected. For Tikhonov method, every singular value is used to estimate the parameter $\boldsymbol{K}$, although the smaller singular values are modified to provide some information and avoid the singular problem at the same time, the bigger singular values are modified accordingly, where the bigger singular values contain more credible information, thus, the estimation of $\boldsymbol{K}$ have some deviations to some extent.As the Tikhonov method dampens all the components which may oversmooth the estimation of $\boldsymbol{K}$, while TSVD excessively dampen components with bigger indexes. To solve the singular problem and include more accurate information for the estimation at same 
time, a new method is proposed. The singular values are divided into groups , a new filter factor parameter is set differently between them.

$$
f_{i}=\left\{\begin{array}{cc}
1 & 0<i \leq k \\
\gamma \sigma_{i+1} & k<i \leq \widetilde{k} \\
0 & \widetilde{k}<i \leq 9
\end{array}\right.
$$

The estimation of $\boldsymbol{K}$ can be expressed as

$$
\boldsymbol{K}=\sum_{i=1}^{9} f_{i} \frac{\left(\boldsymbol{u}_{i}^{T} \boldsymbol{b}_{\boldsymbol{M}}\right) \boldsymbol{v}_{i}}{\sigma_{i}}=\sum_{i=1}^{k} \frac{\left(\boldsymbol{u}_{i}^{T} \boldsymbol{b}_{\boldsymbol{M}}\right) \boldsymbol{v}_{i}}{\sigma_{i}}+\sum_{i=k+1}^{\widetilde{k}} \frac{\left(\boldsymbol{u}_{i}^{T} \boldsymbol{b}_{\boldsymbol{M}}\right) \boldsymbol{v}_{i}}{\gamma \sigma_{i}}
$$

where $\gamma$ satisfies the inequality

$$
0<\gamma<\frac{2 \sigma_{\widetilde{k}}}{\sigma_{k+1}}
$$

The new filter is designed to obtain an equivalent matrix closer to $\boldsymbol{B}_{M}$, therefore, a better estimation of $\boldsymbol{K}$ can be achieved .

According to Eq (3.22), the estimation of $\boldsymbol{K}$ with TSVD method can be equivalent as

$$
\boldsymbol{B}_{T S V D} \boldsymbol{K}=\boldsymbol{b}_{M}
$$

where $\boldsymbol{B}_{T S V D}$ is the equivalent matrix used to replace the original matrix $\boldsymbol{B}_{M}$. $\boldsymbol{B}_{T S V D}$ can be decomposed as Eq (3.13).

Similarly, according to Eq (3.18), the estimation of $\boldsymbol{K}$ with Tikhonov method can be equivalent as

$$
\boldsymbol{B}_{\text {Tikhonov }} \boldsymbol{K}=\boldsymbol{U} \Sigma_{\text {Tikhonov }} \boldsymbol{V}^{T}=\boldsymbol{b}_{M}
$$

where $\Sigma_{\text {Tikhonov }}=\operatorname{diag}\left(\sigma_{1}+\mu^{2} / \sigma_{1}, \ldots, \sigma_{9}+\mu^{2} / \sigma_{9}\right)$. The estimation of $\boldsymbol{K}$ with improved method can be equivalent as

$$
\boldsymbol{B}_{\text {Improved }}=\boldsymbol{U} \Sigma_{\text {Improved }} \boldsymbol{V}^{T}=\boldsymbol{b}_{M}
$$

where $\Sigma_{\text {Improved }}=\operatorname{diag}\left(\sigma_{1}, \ldots, \sigma_{k}, \gamma \sigma_{k+1}, \ldots, \gamma \sigma_{k+1}, 0, \ldots, 0\right)$ It can be proved $\boldsymbol{B}_{\text {Improved }}$ is closer to $\boldsymbol{B}_{M}$ than $\boldsymbol{B}_{\text {Tikhonov }}$ and $\boldsymbol{B}_{T S V D}$ from the spectral and Frobenius norm under some conditions. It is well known if $\|\cdot\|$ is a unitarily invariant norm, then 


$$
\left\|\boldsymbol{B}_{M}\right\|=\left\|\boldsymbol{U} \Sigma \boldsymbol{V}^{T}\right\|=\|\Sigma\|
$$

For the spectral norm, the distance between $\boldsymbol{B}_{M}$ and the equivalent ones can be determined from $\mathrm{Eq}$ (3.30) to Eq (3.32).

$$
\left\|\boldsymbol{B}_{M}-\boldsymbol{B}_{\text {Tikhonov }}\right\|_{2}=\left\|\boldsymbol{U} \Sigma_{M} \boldsymbol{V}^{T}-\boldsymbol{U} \Sigma_{\text {Tikhonov }} \boldsymbol{V}^{T}\right\|_{2}=\left\|\Sigma_{M}-\Sigma_{\text {Tikhonov }}\right\|_{2}=\frac{\mu^{2}}{\sigma_{9}}
$$

$$
\begin{gathered}
\left\|\boldsymbol{B}_{M}-\boldsymbol{B}_{T S V D}\right\|_{2}=\left\|\boldsymbol{U} \Sigma_{M} \boldsymbol{V}^{T}-\boldsymbol{U} \Sigma_{T S V D} \boldsymbol{V}^{T}\right\|_{2}=\left\|\Sigma_{M}-\Sigma_{T S V D}\right\|_{2}=\sigma_{k+1} \\
\begin{aligned}
\left\|\boldsymbol{B}_{M}-\boldsymbol{B}_{\text {Improved }}\right\|_{2} & =\left\|\boldsymbol{U} \Sigma_{M} \boldsymbol{V}^{T}-\boldsymbol{U} \Sigma_{\text {Improved }} \boldsymbol{V}^{T}\right\|_{2}=\left\|\Sigma_{M}-\Sigma_{\text {Improved }}\right\|_{2} \\
& =\max _{k<i<\widetilde{k}}\left|\sigma_{i}-\gamma \sigma_{k+1}\right|
\end{aligned}
\end{gathered}
$$

For the Frobenius norm, the distance between $\boldsymbol{B}_{M}$ and the equivalent ones can be determined from $\mathrm{Eq}$ (3.33) to $\mathrm{Eq}$ (3.35).

$$
\begin{aligned}
\left\|\boldsymbol{B}_{M}-\boldsymbol{B}_{\text {Tikhonov }}\right\|_{F} & =\left\|\boldsymbol{U} \Sigma_{M} \boldsymbol{V}^{T}-\boldsymbol{U} \Sigma_{\text {Tikhonov }} \boldsymbol{V}^{T}\right\|_{F}=\left\|\Sigma_{M}-\Sigma_{\text {Tikhonov }}\right\|_{F} \\
& =\sum_{i=k+1}^{9} \sqrt{\sigma_{i}^{2}} \\
\left\|\boldsymbol{B}_{M}-\boldsymbol{B}_{T S V D}\right\|_{F} & =\left\|\boldsymbol{U} \Sigma_{M} \boldsymbol{V}^{T}-\boldsymbol{U} \Sigma_{T S V D} \boldsymbol{V}^{T}\right\|_{F}=\left\|\Sigma_{M}-\Sigma_{T S V D}\right\|_{F} \\
& =\sum_{i=k+1} \sqrt{\left(\sigma_{i}-\gamma \sigma_{k+1}\right)}+\sum_{i=\widetilde{k}}^{9} \sqrt{\sigma_{i}^{2}} \\
\left\|\boldsymbol{B}_{M}-\boldsymbol{B}_{\text {Improved }}\right\|_{F} & =\left\|\boldsymbol{U} \Sigma_{M} \boldsymbol{V}^{T}-\boldsymbol{U} \Sigma_{\text {Improved }} \boldsymbol{V}^{T}\right\|_{F}=\left\|\Sigma_{M}-\Sigma_{\text {Improved }}\right\|_{F} \\
& =\sum_{i=1}^{9} \sqrt{\left(\frac{\mu^{2}}{\sigma_{i}}\right)^{2}}
\end{aligned}
$$


The reasonable value for $\mu$ with Tikhonov method ranges from $\sigma_{k+1}$ to $\sigma_{k}$, then the inequality (3.36) can be obtained

$$
\frac{\mu^{2}}{\sigma_{9}} \geq \frac{\mu^{2}}{\sigma_{k+1}}=\frac{\mu}{\sigma_{k+1}} \mu \geq \sigma_{k+1}
$$

Thus

$$
\left\|\boldsymbol{B}_{M}-\boldsymbol{B}_{T S V D}\right\|_{2} \leq\left\|\boldsymbol{B}_{M}-\boldsymbol{B}_{\text {Tikhonov }}\right\|_{2}
$$

Since $\frac{\mu^{2}}{\sigma_{i}}=\frac{\mu}{\sigma_{i}} \mu \geq \sigma_{i}$ when $i>k$, inequality (38) can be gotten

$$
\left\|\boldsymbol{B}_{M}-\boldsymbol{B}_{\text {Tikhonov }}\right\|_{F}>\sum_{i=k+1}^{9} \sqrt{\left(\frac{\mu^{2}}{\sigma_{i}}\right)^{2}} \geq \sum_{i=k+1}^{9} \sqrt{\sigma_{i}^{2}}=\left\|\boldsymbol{B}_{M}-\boldsymbol{B}_{T S V D}\right\|_{F}
$$

According to inequality (3.25), inequality (3.39) can be obtained when $k<$ $i \leq \widetilde{k}$

$$
\left|\sigma_{i}-\gamma \sigma_{k+1}\right| \leq \sigma_{i}
$$

Inequality (3.40) can be easily deduced from inequality (3.39)

$$
\left\{\begin{aligned}
\left\|\boldsymbol{B}_{M}-\boldsymbol{B}_{\text {Improved }}\right\|_{2} & \leq\left\|\boldsymbol{B}_{M}-\boldsymbol{B}_{T S V D}\right\|_{2} \\
\left\|\boldsymbol{B}_{M}-\boldsymbol{B}_{\text {Improved }}\right\|_{F} & \leq\left\|\boldsymbol{B}_{M}-\boldsymbol{B}_{T S V D}\right\|_{F}
\end{aligned}\right.
$$

It can be seen the improved method provides a nearer matrix to $\boldsymbol{B}_{M}$ under Frobenius norm and spectral norm. After Eq (3.21) is derived, the most important step is to determine the parameter $k$ and $\lambda$.

\subsubsection{L-curve method for the regularized parameter}

It is essential to confirm the regularized parameter $k$ for the improve TSVD methods, L-curve method is a generally adopted method to select $k$. L-curve is a plot of the norm $\|\boldsymbol{K}\|_{2}$ of the regularized estimation versus the corresponding residual norm $\left\|\boldsymbol{B}_{\boldsymbol{M}} \boldsymbol{K}-\boldsymbol{b}_{\boldsymbol{M}}\right\|_{2}$. Let $\eta=\log \left(\|\boldsymbol{K}\|_{2}\right), \rho=\log \left(\| \boldsymbol{B}_{\boldsymbol{M}} \boldsymbol{K}-\right.$ $\left.\boldsymbol{b}_{\boldsymbol{M}} \|_{2}\right), \eta^{\prime}, \rho^{\prime}, \eta^{\prime \prime}, \rho^{\prime \prime}$ denote the first and the second derivatives of $\eta, \rho$ with respect to $k$. Then the curvature of the curve $\kappa$ is given by

$$
\kappa=2 \frac{\rho^{\prime} \eta^{\prime \prime}-\rho^{\prime \prime} \eta^{\prime}}{\left(\left(\rho^{\prime}\right)^{2}+\left(\eta^{\prime}\right)^{2}\right)^{3 / 2}}
$$

The $k$ corresponding to the maximum value of $\kappa$ is the solution need to be solved. After $k$ is estimated, the parameter $\lambda$ is determined accordingly which is often set between two singular values $\sigma_{k+1} \leq \lambda \leq \sigma_{k}$. 


\subsection{EXPERIMENTS}

\subsubsection{Experiment Setups}

The experimental system includes a TAM, a self-made 24-bit data acquisition system (DAQ) with $1 \mathrm{KHz}$ sampling rate, a notebook computer, a proton magnetometer, a portable power supply, a small vehicle. The TAM to be calibrated is provided by Xi' an Hua Shun company, the internal noise of the TAM is less than $10 p T r m s / \sqrt{\mathrm{Hz}}$ at $1 \mathrm{~Hz}$, the detailed specifications are shown in Table I. The DAQ is used to record the magnetic field data which can be saved to its internal memory card. The GSM-19T proton magnetometer is used to get the geomagnetic field. The TAM is mounted on the top of the vehicle. The notebook computer is used to process the data. The portable $5 \mathrm{~V}$ power supply is used to supply the TAM and the DAQ.

Table 3.1: Specifications of TAM

\begin{tabular}{cccc}
\hline Index & Values & Index & Values \\
\hline Noise & $\leq 10 \mathrm{pT} / \mathrm{Hz}^{1 / 2}$ & Nonorthogonality & $\leq 0.4^{\circ}$ \\
sensitivity & $40 \mu \mathrm{H} / \mathrm{HT}$ & Bandwidth & DC $\sim 50 \mathrm{~Hz}(-3 \mathrm{~dB})$ \\
\hline
\end{tabular}

\subsubsection{Experiment of Calibration}

Firstly, find a experimental site with small magnetic field variation with the proton magnetometer and set the average value as the true scalar geomagnetic field. The accuracy of the scalar reference is higher than $0.1 \mathrm{nT}$. Secondly, fix the TAM on the top of the vehicle and put DAQ, portable battery in the vehicle. Power up the system for 30 minutes to get steady outputs of TAM. Thirdly, drive the vehicle for an entire loop, get the data from the DAQ and calibrate it with the propsoed method. 


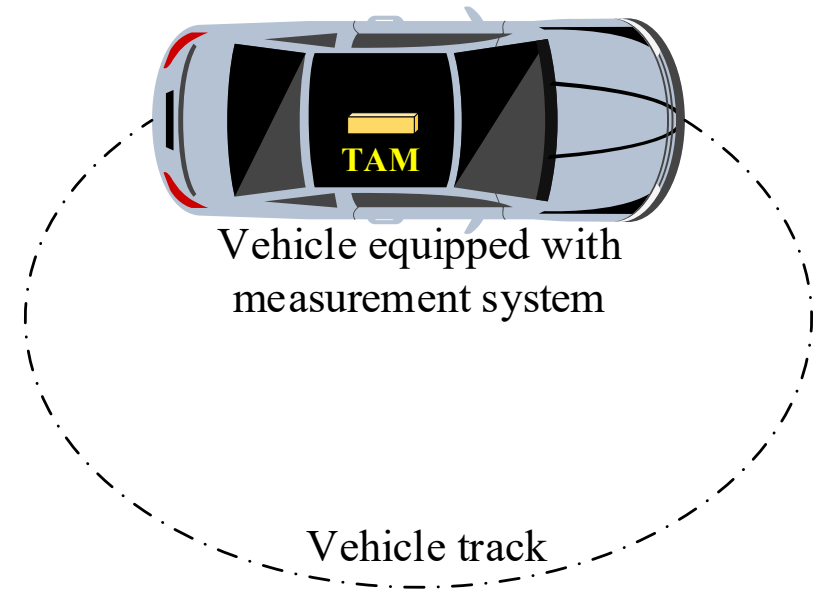

Figure 3.1: Track of vehicle equipped with measruement system

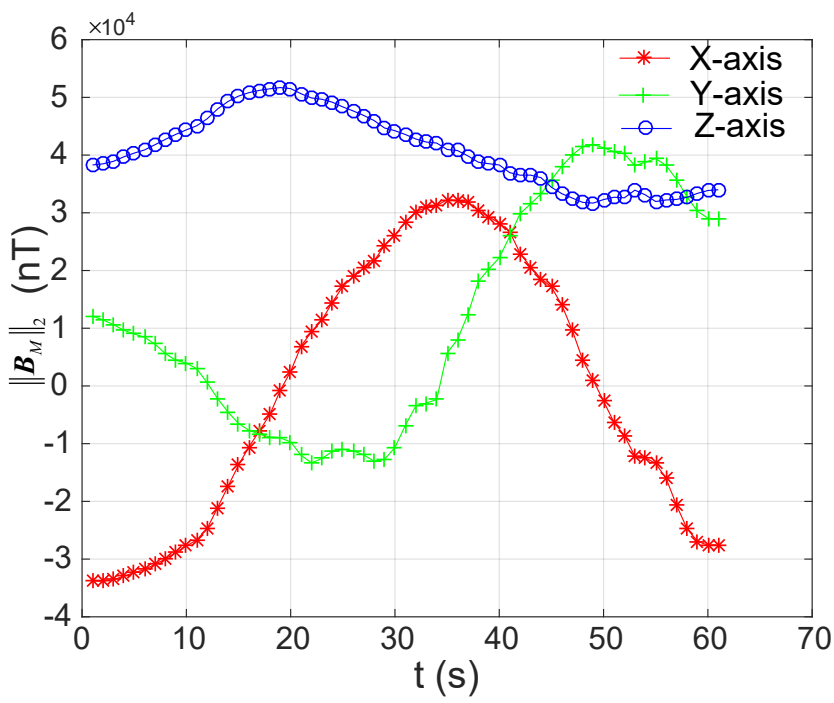

Figure 3.2: Measurements of the TAM during the calibration experiments

It can be seen from Figure (3.2), the magnetic field varies least along the $\mathrm{Z}$-axis, since $\mathrm{Z}$-axis of TAM was set as vertical in the install installation, pitch angle and roll angle vary little during the process.

L-curve method is always try to locate the "corner" of the L-curve, It can be seen the $6^{\text {th }}$ singular value is at the corner of the L-curve from Figure (3.3). Thus, the regularized parameter $k$ is set as $k=6, \lambda$ is set as 
3 Calibration of magnetometers based on improved SVD method

Table 3.2: Average and deviation of the scalar value with different methods

\begin{tabular}{ccccc}
\hline & $\begin{array}{c}\text { Reference } \\
\text { value }\end{array}$ & Raw data & $\begin{array}{c}\text { TTLS } \\
\text { method }\end{array}$ & $\begin{array}{c}\text { Improved } \\
\text { TSVD method }\end{array}$ \\
\hline Avarage (nT) & 52478 & 52886 & 52481 & 52483 \\
Deviation & - & 480020 & 90 & 37 \\
\hline
\end{tabular}

$\lambda=\left(\sigma_{6}+\sigma_{7}\right) / 2$ accordingly. After $k$ and $\lambda$ are determined, all the singular values are used to estimate the error parameters according to $\mathrm{Eq} \mathrm{(3.21)} \mathrm{and}$ Eq (3.5). Subfigure (b) is a local enlargement of subfigure (a) in Figure (3.4), it can be seen the average value of calibrated data with two-step method deviates from the geomagnetic field (about 500nT), the calibration is assumed divergent in this case. The two-step method could not get satisfactory performance because it requires enough measuring points widely spread on the ellipsoid which can not be achieved in this situation. The calibrated results with TTLS and improved TSVD methods are close to the local geomagnetic field. It can be seen the calibrated data with improved TSVD method has less variance than TTLS method, which means it is more suitable for the calibration because the contribution of smaller singular values is considered.

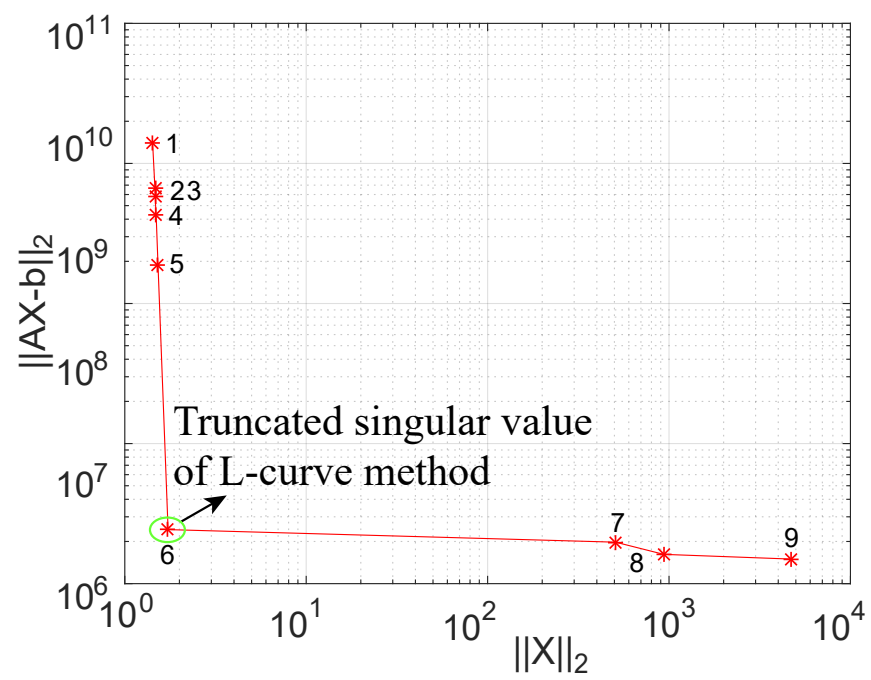

Figure 3.3: Estimated truncated singular value for improved TSVD method 


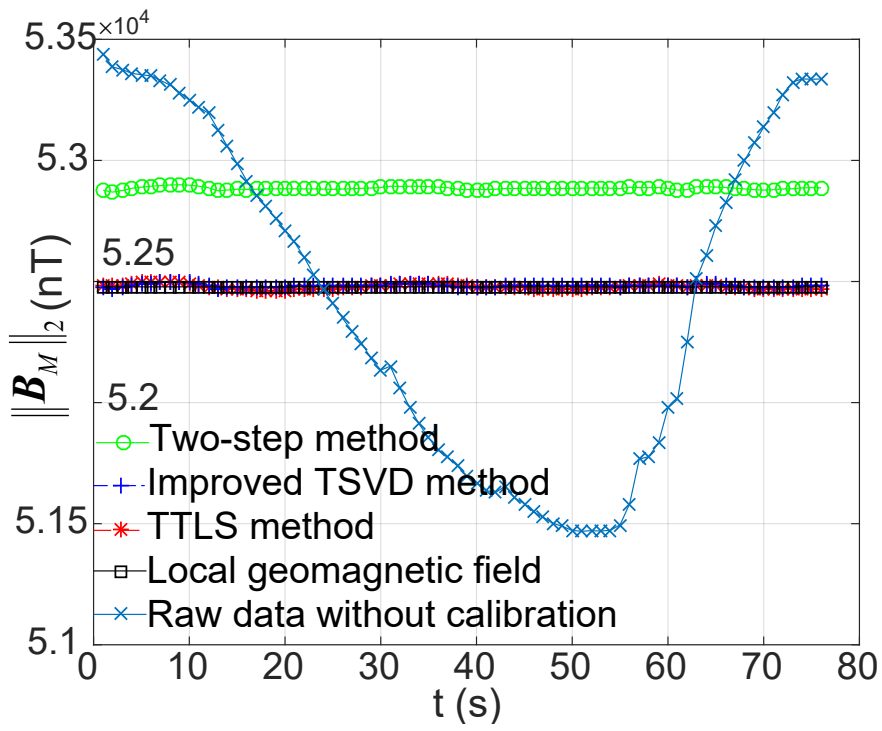

(a) Output of TAM after calibration

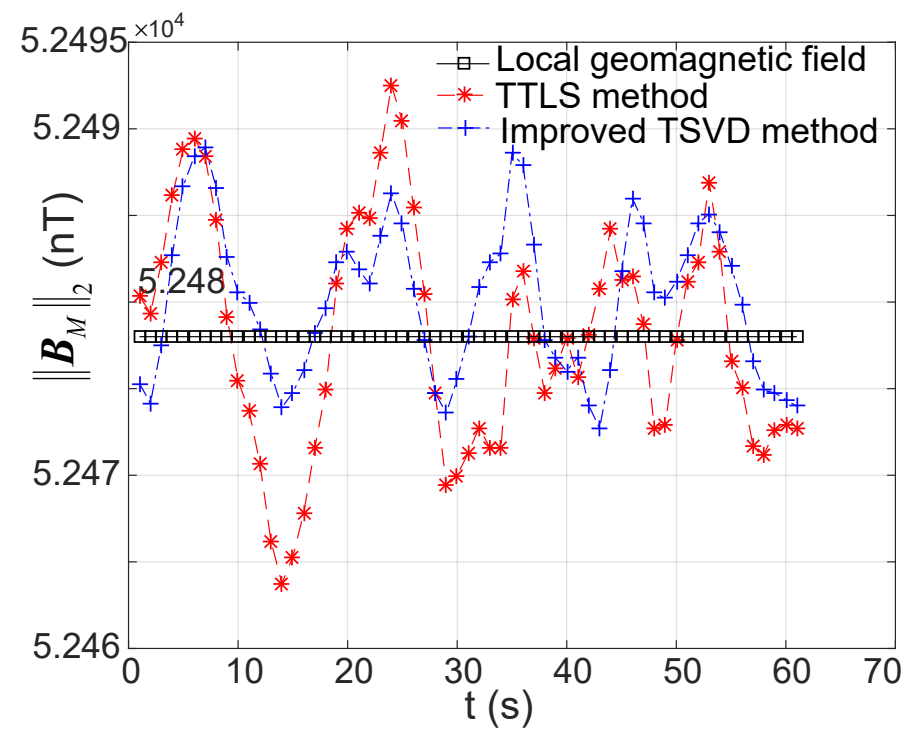

(b) Calibration performance of TTLS and improved TSVD method

Figure 3.4: Results with different scalar calibration method for three-axis magnetometers 


\subsection{CONCLUSION}

The outputs of TAM are distorted by different kinds of errors, thus a calibration must be performed prior to its use. In this paper, we are mainly concentrate on the calibration of the TAM fixed on an object with restricted movement. The traditional methods need abundant outputs of TAM which are widely distributed over an ellipsoid, otherwise, the calibration algorithms often fail. An improved TSVD method was proposed to solve this problem. Firstly the singular values are divided into groups by L-curve method, then the smaller singular values are modified to avoid the divergent situation while the bigger singular values stay the same, the error parameters are estimated and the data is calibrated at last. The experimental results show, the improved TSVD method performs a better calibration performance than the TTLS method in reference [90] while traditional method is divergent. 
CHAPTER 4

\section{Improvement of optical tracking-based orientation estimation by fusing gyroscope information}

This chapter is published as a journal article: Yang, Z., Yan, S., van Beijnum, B. J. F., Li, B., \& Veltink, P. H. (2021). Improvement of Optical Tracking-Based Orientation Estimation by Fusing Gyroscope Information. IEEE Transactions on Instrumentation and Measurement, 70, 1-13. 


\title{
Improvement of optical tracking-based orientation estimation by fusing gyroscope information
}

\begin{abstract}
Optical tracking systems (OTS) can provide high position accuracy over a large workspace. However, The orientation from the OTS is related to the distance between markers, which contains large errors when the distance is small or line-of-sight problems occur. The orientation estimation with a gyroscope is complementary to the OTS-based orientation. In this paper, an Error-state Kalman Filter (ESKF) is proposed to fuse them.

Two experiments were performed to verify the performance: firstly, an unit including markers and a gyroscope was placed statically and rotated dynamically in 3D space. Secondly, two units were used to estimate the relative orientation between the hand and fingers. The static and dynamic orientation error reduced from $0.39^{\circ} \pm 0.16^{\circ}$ and $2.75^{\circ} \pm 1.56^{\circ}$ to $0.23^{\circ} \pm 0.02^{\circ}$ and $1.50^{\circ} \pm 0.62^{\circ}$ respectively, when the distance between markers was $13 \mathrm{~mm}$. The second experimental results show that the fused method improved the OTS performance by smoothing the estimate, filling the relative orientation during the line of sight period, and correcting the estimation when there were identification problems of markers.
\end{abstract}




\subsection{Introduction}

Optical tracking systems (OTS) have become increasingly powerful tools in many biomedical areas, such as biomechanics, gait analysis and sports performance evaluation [93, 94, 95]. An OTS is composed of a set of highspeed cameras and reflective markers or infrared emitting diode markers. Compared with alternative motion tracking systems, such as electromagnetic systems and systems based on inertial measurement units (IMU) or inertial and magnetic measurement units (IMMU), the biggest advantage of OTS is high position estimation accuracy. Therefore, OTS is often used as 'gold standard' for position estimation [96, 97, 98]. However, OTS requires a cluster of at least three markers on a body segment for 3D orientation estimation. The cluster determines a frame of relative marker positions in 3D space and thus a 3D orientation. The accuracy of such OTS-based orientation estimation is limited by two factors, being the relative position accuracy and line-of-sight occlusion. The relative marker position accuracy depends on the accuracy of the marker position estimates and the distances between the markers. The orientation estimate for a body segment which is large and rigid can be adequately accurate, since the marker cluster can be spatially extended. However, OTS-based orientation estimation may be inaccurate if body segments are non-rigid or small, as is the case for hand and fingers. The second factor which may impede OTS-based 3D orientation estimation is 'line of sight' problems due to occlusion.

An alternative popular option for orientation estimation is an IMMUbased system [40, 99, 100]. Rate gyroscope, contained in an IMMU, measure angular velocity directly, from which change of orientation over time can be determined by integration over time. Such estimation can only be trustful over a short period of time, because of integration drift. Full 3D orientation can be estimated and integration drift cancelled if orientation information from angular velocity sensors is combined with complementary information provided by accelerometers and magnetometers, which are also contained in IMMU systems. In this manner, a rather accurate orientation estimate without drift can be obtained [101, 102]. Heading of IMMU-based 3D orientation estimation can, however, easily be disturbed by magnetic disturbances that may especially occur in indoor environments [103, 104]. In addition, inclination estimates may be disturbed if non-gravitational acceleration is not negligible compared with gravitational acceleration. Finally, unlike OTS, estimates of change of 3D position using IMMU systems show 
large drift over time due to the integration operations involved in strapdown navigation, while position estimation is often important in addition to orientation estimation [105, 106, 107].

Fusion of the OTS and IMMU-based movement analysis may improve 3D orientation estimation. It may also potentially help in filling occlusion gaps in the OTS-based orientation estimation. Arash et al. alternatively proposed an orientation-based fusion of inertial and Microsoft Kinect sensors for this purpose [108]. Microsoft Kinect sensors exploit a depth camera rather than high-speed cameras and optical markers. The depth information can be used to reconstruct the shape of the object, which can help to estimate the orientation [109, 110, 111]. The advantages of using Kinect sensors are low cost and no marker attachment. However, its accuracy is still not comparable with marker-based OTS. He et al. proposed a 6 DOF motion tracking method based on IMMU and OTS with an augmented reality head-mounted display (ARHMD) [27]. They used the position from OTS and orientation estimate from an IMMU to update the estimated position of each OTS marker and, subsequently improve the orientation estimation. The accuracy in this case is closely related to the orientation estimation errors caused by the non-gravitational acceleration and the magnetic disturbance.

In this paper, we concentrate on improving the accuracy of the OTSbased orientation estimation and filling the orientation data during occlusions. This is of primary interests in many applications, such as hand movement tracking for rehabilitation [112, 113], navigated lateral skull base surgeries, etc. [114]. We propose to estimate 3D orientation by fusing the information from an OTS and a rate gyroscope, without including the information from the accelerometer and the magnetometer in order to avoid the errors they may induce: Firstly, the error caused by the integration drift of linear accelerations extracted from the accelerometer in case change of occluded marker positions would be estimated is eliminated, since only orientation estimates from OTS and gyroscope are fused. Secondly, the orientation errors induced by the non-gravitational acceleration and the magnetic disturbance are eliminated. We demonstrated our proposed method through experimental evaluation. 


\subsection{Methods}

In this section, we firstly describe the orientation error induced from an OTS, and subsequently a gyroscope sensor model and orientation estimation using the gyroscope. Finally, we propose an Error-state Kalman filter (ESKF) and smoothing techniques to fuse the data from the OTS and the gyroscope sensor. For the description below, three different coordinate frames are used:

Sensor frame s - determined by the gyroscope sensor;

Marker frame $\mathrm{m}$ - determined by the optical markers;

Global frame $\mathrm{g}$ - determined by the calibration of the optical system prior to the experiment. Positions of the markers are measured in this frame and also the quaternion-based orientation is determined relative to this frame.

\subsubsection{Error analysis of orientation estimation using an OTS}

Orientation estimation using OTS is commonly based on position measurements of a cluster of markers. As shown in Fig 4.1, positions of three markers are measured as:

$$
\left\{\begin{array}{c}
\boldsymbol{y}_{m i}^{g}=\boldsymbol{p}_{i}^{g}+\delta \boldsymbol{p}_{i}^{g}(i=1,2,3) \\
\delta \boldsymbol{p}_{i}^{g} \sim\left(0, \sigma_{m}\right)
\end{array}\right.
$$

where $\boldsymbol{p}_{i}^{g}$ is the true position of the marker and $\delta \boldsymbol{p}_{i}^{g}$ is the measurement

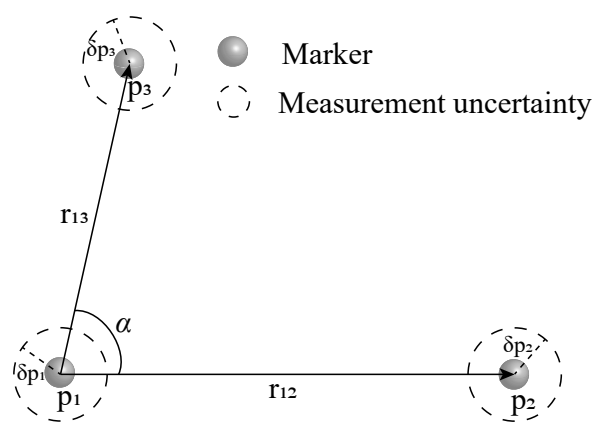

Figure 4.1: Illustration of a marker cluster used for orientation estimation with measurement errors. The positions of markers are $\boldsymbol{p}_{i}(i=$ $1,2,3)$ with standard deviation $\sigma_{m}$, which will cause error for relative position $\boldsymbol{r}_{12}$ and $\boldsymbol{r}_{13}$, and subsequently cause errors for the orientation estimation.

error, which is assumed to have a normal distribution with zero mean and 
standard deviation $\sigma_{m} \cdot \boldsymbol{y}_{m i}^{g}$ is the marker position measured by the OTS. All parameters are in the global frame. The relative position between markers is calculated as

$$
\hat{\boldsymbol{r}}_{i j}^{g}=\boldsymbol{y}_{m j}^{g}-\boldsymbol{y}_{m i}^{g}=\boldsymbol{r}_{i j}^{g}+\delta \boldsymbol{p}_{i j}^{g}(j=2,3 ; i=1)
$$

where $\hat{\boldsymbol{r}}_{i j}^{g}$ is the relative position obtained from OTS measurements, $\boldsymbol{r}_{i j}^{g}$ is the true relative position, $\delta \boldsymbol{p}_{i j}^{g}$ is the measurement error

$$
\left\{\begin{array}{c}
\boldsymbol{r}_{i j}^{g}=\boldsymbol{p}_{j}^{g}-\boldsymbol{p}_{i}^{g} \\
\delta \boldsymbol{p}_{i j}^{g}=\delta \boldsymbol{p}_{j}^{g}-\delta \boldsymbol{p}_{i}^{g}, \delta \boldsymbol{p}_{i j}^{g} \sim\left(0, \sqrt{2} \boldsymbol{\sigma}_{m}\right)
\end{array}\right.
$$

Based on positions of three markers, we can obtain two vectors $\hat{\boldsymbol{r}}_{12}^{g}$ and $\hat{\boldsymbol{r}}_{13}^{g}$. Subsequently, The orientation based on optical markers can be estimated as follows. More details are described in Appendix A.4.

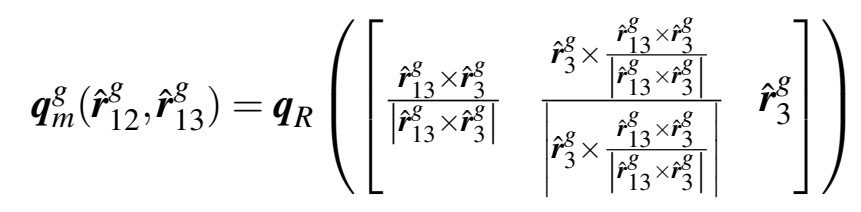

where $\boldsymbol{q}_{R}$ is the transfer function from rotation matrix to quaternion. $\hat{\boldsymbol{r}}_{3}^{g}$ is

$$
\hat{\boldsymbol{r}}_{3}^{g}=\frac{\hat{\boldsymbol{r}}_{12}^{g}}{\left|\hat{\boldsymbol{r}}_{12}^{g}\right|}
$$

Based on Fig 4.1 and Eq (4.4), we find that the error of relative orientation $\boldsymbol{q}_{m}^{g}\left(\hat{\boldsymbol{r}}_{12}^{g}, \hat{\boldsymbol{r}}_{13}^{g}\right)$ is related to $\alpha, \delta \boldsymbol{p}_{i}$ and $\boldsymbol{p}_{i}$, as described by the the following equation

$$
\boldsymbol{q}_{\text {err }}=\left[\boldsymbol{q}_{m}^{g}\left(\hat{\boldsymbol{r}}_{12}^{g}, \hat{\boldsymbol{r}}_{13}^{g}\right)\right]^{-1} \otimes \boldsymbol{q}_{m}^{g}\left(\boldsymbol{r}_{12}^{g}, \boldsymbol{r}_{13}^{g}\right)
$$

where $\otimes$ represents multiplication between quaternions. The error angle $\left|\boldsymbol{\theta}_{\text {err }}\right|$ is determined by $\boldsymbol{q}_{\text {err }}$. As $\boldsymbol{r}_{12}^{g}$ and $\boldsymbol{r}_{13}^{g}$ are close to $\hat{\boldsymbol{r}}_{12}^{g}$ and $\hat{\boldsymbol{r}}_{13}^{g}$, the rotation error $\boldsymbol{q}_{\text {err }}$ is quite small, and can, therefore, be approximated as

$$
\boldsymbol{q}_{\text {err }} \approx\left[\begin{array}{ll}
1 & \frac{1}{2} \boldsymbol{\theta}_{\text {err }}
\end{array}\right]
$$

In order to investigate the orientation error $\boldsymbol{\theta}_{\text {err }}$ caused by $\alpha$ and $\delta \boldsymbol{p}_{i}$ for varying $\left|\boldsymbol{r}_{i j}\right|$, a simulation-based Monte Carlo analysis was performed. $\delta \boldsymbol{p}_{i}$ 
was assumed to have normal distribution with zero mean and standard deviation of $\sigma_{m}$. Fig 4.2 shows the simulation results, which indicate that the orientation error increases when $\sigma_{m}$ becomes larger or $\alpha$ smaller. More details can be found in Table II in Appendix A.1. The error source $\alpha$ is determined by the configuration of markers. Subfigure (b) of Fig 4.2, indicates that the error induced by $\alpha$ can be minimized by designing $\boldsymbol{r}_{12}$ and $\boldsymbol{r}_{13}$ perpendicular to each other $(\alpha=\pi / 2)$. The error source $\delta p_{i}$, it can be reduced by reducing the distance between the cameras and the markers, or by changing the configuration or number of cameras. Given a certain OTS setup, the influence of $\delta \boldsymbol{p}_{i}$ can be reduced by increasing marker distance $\left|\boldsymbol{r}_{i j}\right|$. However, the space for attachment of markers is quite small for some applications, such as tracking of finger movements.

\subsubsection{Sensor model}

The gyroscope sensor measures angular velocity in sensor frame, disturbed by various errors, including gain error, non-orthogonality error and offset error. After sensor calibration, gain and non-orthogonality error can be eliminated. However, the offset error is a slowly varying variable and has a large accumulating effects when integrating angular velocity to orientation change. Thus, it is estimated as part of the sensor model. The error model of a gyroscope can be simplified as [102]:

$$
\boldsymbol{y}_{g y r}^{s}=\boldsymbol{\omega}^{s}-\boldsymbol{b}^{s}-\boldsymbol{e}^{s}
$$

where $\boldsymbol{y}_{\text {gyr }}^{s}$ is the gyroscope sensor output, $\boldsymbol{\omega}^{s}$ is the corrected angular velocity in sensor frame, $\boldsymbol{b}^{s}$ and $\boldsymbol{e}^{s}$ are corresponding offset error and measurement noise.

\subsubsection{Orientation estimation based on a gyroscope}

From a gyroscope, change of orientation over time can be estimated by integrating angular velocity. Since quaternion representation has high efficiency compared with rotation matrices and Euler angles, we use quaternions for describing 3D orientation and orientation update

$$
\boldsymbol{q}_{g, k+1}^{s}=\boldsymbol{q}_{g, k}^{s} \otimes \delta \boldsymbol{q}_{g, k}^{s}
$$




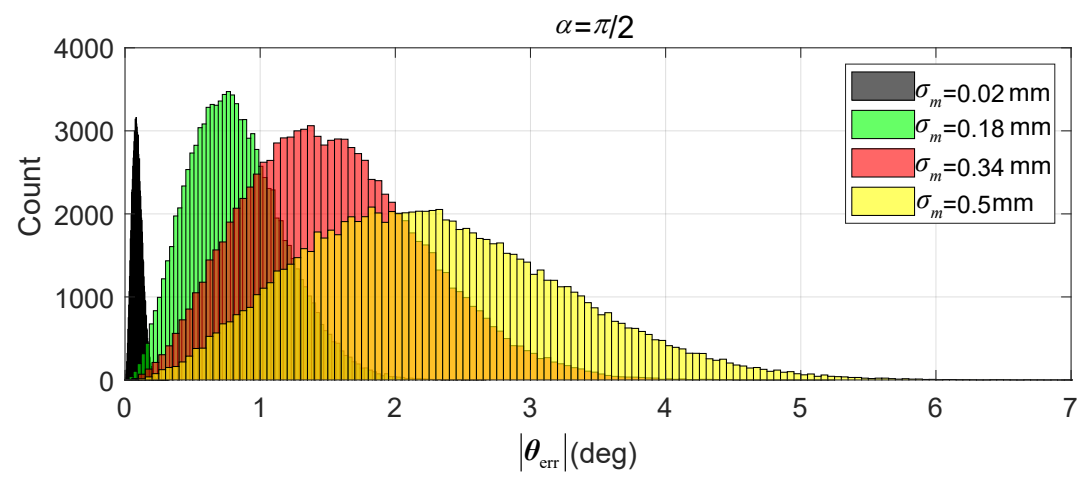

(a)

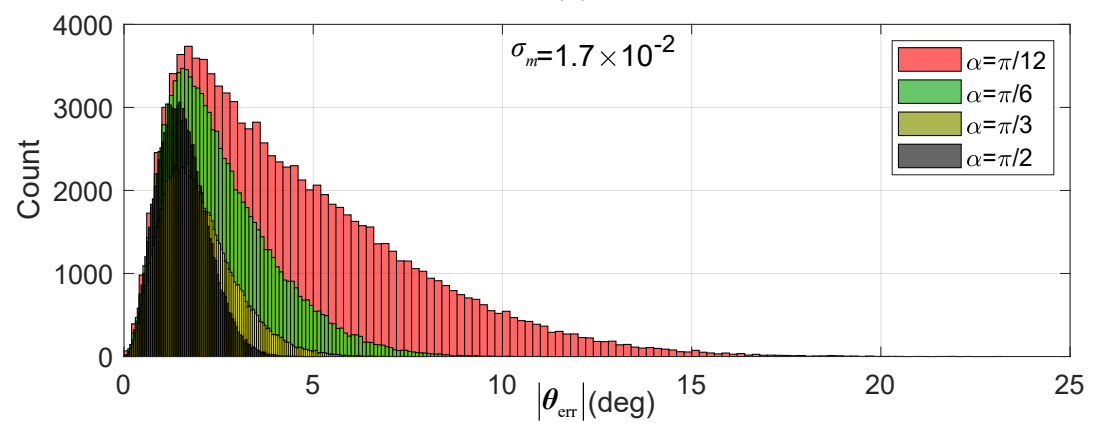

(b)

Figure 4.2: Error angle $\left|\boldsymbol{\theta}_{\text {err }}\right|$ for varying angles $\alpha$ and measurement noise $\delta p_{i} . \delta p_{i}$ and $\alpha$ are investigated based on Monte Carlo analysis, where $\alpha$ is the angle between two vectors. (see Fig 4.1 and Eq (4.1)). Distance $\left|\boldsymbol{r}_{12}\right|$ and $\left|\boldsymbol{r}_{13}\right|$ are both set as $20 \mathrm{~mm}$, $\delta \boldsymbol{p}_{i}$ was generated under normal distribution $\left(0, \sigma_{m}\right), 10^{5}$ particles were used in each simulation. (a) shows the distribution of $\left|\boldsymbol{\theta}_{\text {err }}\right|$ with different $\sigma_{m}$, $\alpha$ is set as $\pi / 2$. (b) shows the distribution of orientation error $\left|\boldsymbol{\theta}_{\text {err }}\right|$ with different $\alpha$.

$\boldsymbol{q}_{g, k}^{s}$ is the orientation in global frame at time $k$. If the rotation angle in a time step is small, $\delta \boldsymbol{q}_{g, k}^{s}$ can be approximated as

$$
\delta \boldsymbol{q}_{g, k}^{s} \approx\left[\begin{array}{ll}
1 & \frac{1}{2} \delta \boldsymbol{\theta}_{k}^{s}
\end{array}\right]=\left[\begin{array}{ll}
1 & \frac{1}{2} d_{t} \boldsymbol{\omega}_{k}^{s}
\end{array}\right]
$$

where $d_{t}$ is the time interval between time $k$ and $k+1$. $\boldsymbol{\omega}_{k}^{s}$ is the angular velocity in sensor frame at time $k$. 


\subsubsection{Data fusion based on ESKF}

We propose an ESKF to fuse gyroscope and OTS based orientation estimates. This method has an excellent reputation for human body motion tracking using IMMU $[115,116,117]$. Unlike an Extended Kalman Filter (EKF) that updates only nominal-state, ESKF updates both the nominal and error-state estimates. The true-state is estimated by the sum of nominal and error-states. The nominal and error-states are considered as larger and small state components. The nominal-state does not consider noise terms while the error-state estimate incorporates noise and perturbations. The small error state enables formulation of a linearized error state model and easier computation of Jacobians [118]. The applied ESKF design is shown in Fig 4.3 .

The orientation and offset of gyroscope are included in the state vector. The true- state, nominal-state and error-state are defined as

$$
\left\{\begin{array}{cl}
\boldsymbol{x}=\left(\left(\boldsymbol{q}_{g}^{S}\right)^{T}\right. & \left.\left(\boldsymbol{b}^{S}\right)^{T}\right)^{T} \\
\hat{\boldsymbol{x}}=\left(\left(\hat{\boldsymbol{q}}_{g}^{S}\right)^{T}\right. & \left.\left(\hat{\boldsymbol{b}}^{S}\right)^{T}\right)^{T} \\
\boldsymbol{x}_{\varepsilon}=\left(\boldsymbol{\theta}_{\varepsilon}^{T}\right. & \left.\boldsymbol{b}_{\varepsilon}^{T}\right)^{T}
\end{array}\right.
$$

where $\wedge$ represents nominal-state estimate, $\varepsilon$ represents error-state. $\boldsymbol{q}_{g}^{s}$ and $\boldsymbol{b}^{s}$ are quaternion-based orientation and offset of the gyroscope. $\boldsymbol{\theta}_{\varepsilon}$ and $\boldsymbol{b}_{\varepsilon}$ are error-states related to $\boldsymbol{q}_{g}^{s}$ and $\boldsymbol{b}^{s} . \boldsymbol{q}_{g}^{s}, \hat{\boldsymbol{q}}_{g}^{s}, \boldsymbol{b}^{s}$ and $\hat{\boldsymbol{b}}^{s}$ are abbreviated as $\boldsymbol{q}, \hat{\boldsymbol{q}}, \boldsymbol{b}$ and $\hat{b}$ below.

As shown in Fig 4.3, the update of the orientation $\boldsymbol{q}_{k}$ and gyroscope offset $\boldsymbol{b}_{k}$ includes the update of the error-state and nominal-state. The process can be divided into four steps: the first step is to initialize the orientation and gyroscope offset, including the error-state and nominal-state. The second step is to predict the error-state and nominal-state for the orientation and gyroscope offset. The prediction for the nominal-state is mainly deduced by the integration of angular velocity exploiting Eq (4.9) and (4.10). The prediction for the error-states is mainly deduced by the differential of the relation between the true-state and nominal-state (see Eq (4.23) and (4.24)). The third step is to update the error-state based on measurement data. The relation between measurement data and error-states can be obtained from the described process model that describe the relation between the measurement data and true-state. The fourth step is to obtain a prediction of the true-state based on the prediction of the nominal-state and updated error-state. More details are as follows: 


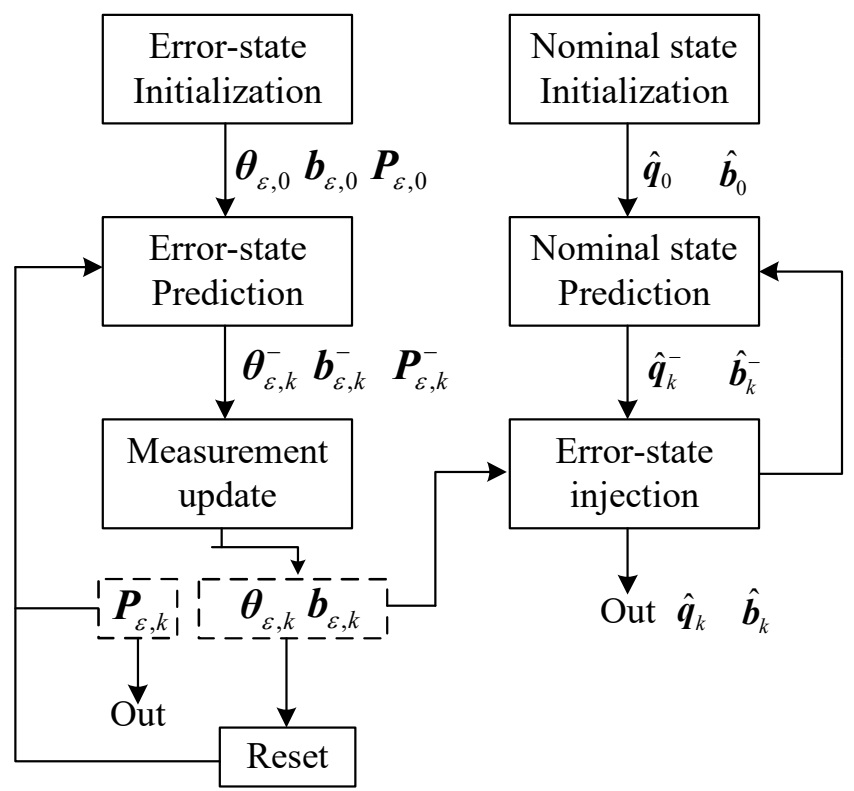

Figure 4.3: ESKF design. The ESKF can be divided into two parts. Firstly, update the error-state parameter $\hat{\boldsymbol{\theta}}_{\varepsilon, 0}$ and $\hat{\boldsymbol{b}}_{\varepsilon, 0}$, as shown on the left side. Secondly, injection of the error-state parameter in the nominal-state estimate and, update of the nominal-state parameter $\hat{\boldsymbol{q}}_{k}$ and $\hat{\boldsymbol{b}}_{k}$, as shown on the right side.

\section{Initialization}

The initial value of the nominal-state $\hat{\boldsymbol{q}}_{0}$ is obtained from the OTS. The initial values for error-states $\hat{\boldsymbol{b}}_{0}, \boldsymbol{\theta}_{\varepsilon, 0}$, and $\boldsymbol{b}_{\varepsilon, 0}$ are set to $\boldsymbol{0}$. Before every prediction and update, we obtain the angular velocity $\boldsymbol{y}_{\text {gyr }}^{s}$ from the gyroscope, and know the nominal-state estimate $\hat{\boldsymbol{x}}_{k-1}$ at time $k-1$, and the covariance matrix $\boldsymbol{P}_{\varepsilon, k-1}$ belonging to estimated error-state $\boldsymbol{x}_{\varepsilon, k-1}$.

\section{Prediction}

The purpose of this step is to predict the nominal-state $\hat{\boldsymbol{x}}_{k}^{-}$, error-state $\boldsymbol{x}_{\varepsilon, k}^{-}$ and the corresponding covariance matrix $\boldsymbol{P}_{\varepsilon, k}^{-}$, where ${ }^{-}$denotes the prior estimation. Nominal-state prediction: The offset error of the gyroscope $\boldsymbol{b}$ is modeled as a first order Markov process driven by white noise $\boldsymbol{n}_{b, k}$

$$
\boldsymbol{b}_{k}=\boldsymbol{b}_{k-1}+\boldsymbol{n}_{b, k}
$$


For prediction of $\boldsymbol{b}$, we assume

$$
\hat{\boldsymbol{b}}_{k}^{-}=\hat{\boldsymbol{b}}_{k-1}
$$

The prediction of orientation based on the gyroscope is performed by using Eq (4.9) and (4.10).

$$
\hat{\boldsymbol{q}}_{k}^{-}=\left(\boldsymbol{I}_{4 \times 4}+\frac{1}{2} \boldsymbol{\Omega}_{k-1} d_{t}\right) \hat{\boldsymbol{q}}_{k-1}
$$

where $\boldsymbol{\Omega}_{k-1}$ is

$$
\boldsymbol{\Omega}_{k-1}=\left[\begin{array}{cccc}
0 & -\hat{\omega}_{x}^{s} & -\hat{\omega}_{y}^{s} & -\hat{\omega}_{z}^{s} \\
\hat{\omega}_{x}^{s} & 0 & \hat{\omega}_{z}^{s} & -\hat{\omega}_{y}^{s} \\
\hat{\omega}_{y}^{s} & -\hat{\omega}_{z}^{s} & 0 & \hat{\omega}_{x}^{s} \\
\hat{\omega}_{z}^{s} & \hat{\omega}_{y}^{s} & -\hat{\omega}_{x}^{s} & 0
\end{array}\right]_{k-1}
$$

and $\hat{\omega}_{x}^{s}, \hat{\omega}_{y}^{s}, \hat{\omega}_{z}^{s}$ are components of nominal angular velocity $\hat{\boldsymbol{\omega}}_{k-1}^{s} \cdot \hat{\boldsymbol{\omega}}_{k}^{s}$ can be obtained according to $\mathrm{Eq}$ (4.8):

$$
\hat{\boldsymbol{\omega}}_{k}^{s}=\boldsymbol{y}_{g y r, k}^{s}+\hat{\boldsymbol{b}}_{k}
$$

The prediction for nominal-state $\hat{\boldsymbol{x}}_{k}$ is given by

$$
\hat{\boldsymbol{x}}_{k}^{-}=\boldsymbol{F}_{k} \hat{\boldsymbol{x}}_{k-1}
$$

where the transition matrix $\boldsymbol{F}_{k}$ is

$$
\boldsymbol{F}_{k}=\left[\begin{array}{cc}
\left(\boldsymbol{I}_{4 \times 4}+\frac{d_{t}}{2} \boldsymbol{\Omega}_{k}\right) & \boldsymbol{0}_{4 \times 3} \\
\boldsymbol{0}_{3 \times 4} & \boldsymbol{I}_{3 \times 3}
\end{array}\right]
$$

Error-state prediction: The error-state of the gyroscope offset $\boldsymbol{b}_{\varepsilon, k}$ is defined as the difference between the estimated and true value of $\boldsymbol{b}_{k}$

$$
\boldsymbol{b}_{\varepsilon, k}=\hat{\boldsymbol{b}}_{k}-\boldsymbol{b}_{k}
$$

If we substitute $\mathrm{Eq}$ (4.12) and $\mathrm{Eq}$ (4.13) in $\mathrm{Eq}$ (4.19), we find:

$$
\boldsymbol{b}_{\varepsilon, k}=\hat{\boldsymbol{b}}_{k}-\boldsymbol{b}_{k}=\hat{b}_{k-1}-\left(\boldsymbol{b}_{k-1}+\boldsymbol{n}_{b, k}\right)=\boldsymbol{b}_{\varepsilon, k-1}-\boldsymbol{n}_{b, k}
$$

The prediction for the error-state $\boldsymbol{b}_{\varepsilon, k}$ is

$$
\boldsymbol{b}_{\varepsilon, k}^{-}=\boldsymbol{b}_{\varepsilon, k-1}
$$


For a small orientation error denoted $\boldsymbol{\theta}_{\varepsilon}$, the corresponding quaternion-based orientation error can be approximated as

$$
\boldsymbol{q}_{\varepsilon} \approx\left[\begin{array}{ll}
1 & \frac{1}{2} \boldsymbol{\theta}_{\varepsilon}
\end{array}\right]
$$

The true quaternion can be expressed as

$$
\boldsymbol{q}=\hat{\boldsymbol{q}} \otimes \boldsymbol{q}_{\varepsilon}
$$

If we differentiate $\mathrm{Eq}$ (4.23) on both sides, we obtain the following equation, more details can be found in the work of Schepers et al. [41] and Weenk et al [119].

$$
\dot{\boldsymbol{\theta}}_{\varepsilon}=-\left\lfloor\hat{\boldsymbol{\omega}}^{s}\right\rfloor_{\times} \boldsymbol{\theta}_{\varepsilon}-\boldsymbol{\omega}_{\varepsilon}^{s}
$$

where $\hat{\boldsymbol{\omega}}^{s}$ can be obtained from Eq (4.16). $\bigsqcup_{\times}$denotes a skew-symmetric matrix. More details can be found in Appendix A.3. $\boldsymbol{\omega}_{\varepsilon}^{s}$ is the difference between the estimated and true angular velocity:

$$
\begin{aligned}
\boldsymbol{\omega}_{\varepsilon}^{s} & =\hat{\boldsymbol{\omega}}^{s}-\boldsymbol{\omega}^{s} \\
& =\boldsymbol{y}_{\text {gyr }}^{s}+\hat{\boldsymbol{b}}-\left(\boldsymbol{y}_{g y r}^{s}+\boldsymbol{b}+\boldsymbol{e}^{s}\right) \\
& =\boldsymbol{b}_{\varepsilon}-\boldsymbol{e}^{s}
\end{aligned}
$$

By combining Eq (4.24) and Eq (4.25), the orientation error can be expressed as

$$
\boldsymbol{\theta}_{\varepsilon, k}=\left(\boldsymbol{I}_{3 \times 3}-\left\lfloor\hat{\boldsymbol{\omega}}_{k-1}^{s}\right\rfloor_{\times} d_{t}\right) \boldsymbol{\theta}_{\varepsilon, k-1}-\left(\boldsymbol{b}_{\varepsilon, k-1}-\boldsymbol{e}^{s}\right) d_{t}
$$

Based on Eq (4.26), the prediction for orientation error $\boldsymbol{\theta}_{\varepsilon, k}$ is updated as

$$
\boldsymbol{\theta}_{\varepsilon, k}^{-}=\left(\boldsymbol{I}_{3 \times 3}-\left\lfloor\hat{\boldsymbol{\omega}}_{k-1}^{s}\right\rfloor_{\times} d_{t}\right) \boldsymbol{\theta}_{\varepsilon, k-1}-\boldsymbol{b}_{\varepsilon, k-1} d_{t}
$$

From Eq (4.20) and (4.26), we can obtain the process model for the errorstate $\boldsymbol{x}_{\varepsilon, k}$

$$
\boldsymbol{x}_{\varepsilon, k}=\boldsymbol{F}_{\varepsilon, k} \boldsymbol{x}_{\varepsilon, k-1}+\boldsymbol{n}_{\boldsymbol{x}_{k}}
$$

where $\boldsymbol{n}_{\boldsymbol{x}_{k}}$ is the noise of the process model. Transition matrix $\boldsymbol{F}_{\boldsymbol{\varepsilon}, k}$ can be expressed as

$$
\boldsymbol{F}_{\varepsilon, k}=\left[\begin{array}{cc}
\frac{\partial\left(\boldsymbol{\theta}_{\varepsilon, k}\right)}{\partial\left(\boldsymbol{\theta}_{\varepsilon, k-1}\right)} & \frac{\partial\left(\boldsymbol{\theta}_{\varepsilon, k}\right)}{\partial \boldsymbol{b}_{\varepsilon, k-1}} \\
\boldsymbol{0}_{3 \times 3} & \boldsymbol{I}_{3 \times 3}
\end{array}\right]
$$

Details of the elements in the first row can be found in Appendix A.3. The final prediction formula for error-state $\boldsymbol{x}_{\varepsilon, k}$ is

$$
\boldsymbol{x}_{\varepsilon, k}^{-}=\boldsymbol{F}_{\varepsilon, k} \boldsymbol{x}_{\varepsilon, k-1}
$$


The covariance matrix $\boldsymbol{P}$ is updated using

$$
\boldsymbol{P}_{\varepsilon, k}^{-}=\boldsymbol{F}_{\varepsilon, k} \boldsymbol{P}_{\varepsilon, k-1} \boldsymbol{F}_{\varepsilon, k}^{T}+\boldsymbol{Q}_{\varepsilon}
$$

where $\boldsymbol{Q}_{\varepsilon}$ is the noise covariance matrix of $\boldsymbol{n}_{\boldsymbol{x}_{k}}$.

\section{Measurement update}

From the OTS, we obtain the orientation from marker frame to global frame $\boldsymbol{q}_{m}^{g}$ based on Eq (4.4). Thus, the orientation from global frame to marker frame $\boldsymbol{q}_{g}^{m}$ is known. $\boldsymbol{y}_{k}$ can be expressed as

$$
\boldsymbol{y}_{k}=\left(\boldsymbol{q}_{g}^{m}\right)_{k}+\boldsymbol{n}_{\boldsymbol{y}_{k}}=\left(\boldsymbol{q}_{s}^{m}\right)_{k} \otimes\left(\boldsymbol{q}_{g}^{s}\right)_{k}+\boldsymbol{n}_{\boldsymbol{y}_{k}}
$$

where $\boldsymbol{q}_{s}^{m}$ is the orientation from sensor frame to marker frame, which can be estimated from calibration movements prior the experiment. Eq (4.32) can be simplified as

$$
\hat{\boldsymbol{y}}_{k}=\left(\boldsymbol{q}_{g}^{s}\right)_{k}+\boldsymbol{n}_{\boldsymbol{y}_{k}}
$$

where $\hat{\boldsymbol{y}}_{k}$ is the estimation of $\boldsymbol{y}_{k}$ from measurement data

$$
\hat{\boldsymbol{y}}_{k}=\left(\boldsymbol{q}_{s}^{m}\right)_{k}^{-1} \otimes \boldsymbol{y}_{k}
$$

Based on Eq (4.22) and (4.23), Eq (4.33) can be expressed as

$$
\hat{\boldsymbol{y}}_{k}=\left(\hat{\boldsymbol{q}}_{g}^{S}\right)_{k} \otimes\left[\begin{array}{ll}
1 & \frac{1}{2} \boldsymbol{\theta}_{\boldsymbol{\varepsilon}, k}
\end{array}\right]+\boldsymbol{n}_{\boldsymbol{y}_{k}}
$$

Based on Eq (4.35), the measurement model can be updated as

$$
\hat{\boldsymbol{y}}_{k}=\boldsymbol{H}_{\varepsilon, k} \boldsymbol{x}_{\varepsilon, k}+\boldsymbol{n}_{\boldsymbol{y}_{k}}
$$

where $\boldsymbol{H}_{\varepsilon, k}$ is the Jacobian matrix of $\hat{\boldsymbol{y}}_{k}$. Details can be found in the Appendix A.3.

$$
\boldsymbol{H}_{\varepsilon, k}=\left[\begin{array}{ll}
\frac{\partial \hat{\boldsymbol{y}}_{k}}{\partial\left(\boldsymbol{\theta}_{\varepsilon, k}\right)} & \frac{\partial \hat{y}_{k}}{\partial\left(\boldsymbol{b}_{\varepsilon, k}\right)}
\end{array}\right]
$$

Subsequently, we can update the Kalman gain $\boldsymbol{K}_{\varepsilon, k}$

$$
\boldsymbol{K}_{\varepsilon, k}=\boldsymbol{P}_{\varepsilon, k}^{-} \boldsymbol{H}_{\varepsilon, k}^{T}\left(\boldsymbol{H}_{\varepsilon, k} \boldsymbol{P}_{\varepsilon, k}^{-} \boldsymbol{H}_{\varepsilon, k}^{T}+\boldsymbol{R}_{\varepsilon}\right)^{-1}
$$

where $\boldsymbol{R}_{\boldsymbol{\varepsilon}}$ is the measurement noise variance related to error $\boldsymbol{n}_{\boldsymbol{y}_{k}}$. The prior error-state $\boldsymbol{x}_{\varepsilon, k}^{-}$is updated based on the Kalman gain above

$$
\boldsymbol{x}_{\varepsilon, k}=\boldsymbol{x}_{\varepsilon, k}^{-}+\boldsymbol{K}_{\varepsilon, k}\left(\hat{\boldsymbol{y}}_{k}-\boldsymbol{H}_{\varepsilon, k} \boldsymbol{x}_{\varepsilon, k}^{-}\right)
$$


The error covariance matrix $\boldsymbol{P}_{\varepsilon, k}^{-}$is updated using

$$
\boldsymbol{P}_{\varepsilon, k}=\left(\boldsymbol{I}-\boldsymbol{K}_{\varepsilon, k} \boldsymbol{H}_{\varepsilon, k}\right) \boldsymbol{P}_{\varepsilon, k}^{-}
$$

\section{Injection of the observed error into the nominal-state}

Based on Eq (4.19), the two components of $\boldsymbol{x}_{\varepsilon, k}$ are, $\boldsymbol{b}_{\varepsilon, k}$ and $\boldsymbol{\theta}_{\varepsilon, k} . \boldsymbol{b}_{k}$ can be updated as:

$$
\hat{\boldsymbol{b}}_{k}=\hat{\boldsymbol{b}}_{k}^{-}-\boldsymbol{b}_{\varepsilon, k}
$$

The orientation $\hat{\boldsymbol{q}}_{k}$ from Eq (4.24) can be corrected from the $\boldsymbol{\theta}_{\varepsilon, k}$

$$
\hat{\boldsymbol{q}}_{k}=\hat{\boldsymbol{q}}_{k}^{-} \otimes\left[\begin{array}{ll}
1 & \frac{1}{2} \boldsymbol{\theta}_{\varepsilon, k}
\end{array}\right]
$$

After the nominal-state is corrected, the error-state is reset for the next iteration.

\subsubsection{Smoothing}

In many biomedical applications, the full measurement sequence is available and the data can be analyzed offline [120, 121, 122]. Smoothing technique can be used to improve the accuracy of state estimation. For the ESKF mentioned above, the state vector $\boldsymbol{x}_{k}$ is estimated based on the measurement from $\boldsymbol{y}_{1}$ to $\boldsymbol{y}_{k}$, satisfying the causality requirement for real-time processing. With the offline smoothing technique, $\boldsymbol{x}_{k}$ can be estimated non-causally with all the measurements from $\boldsymbol{y}_{1}$ to $\boldsymbol{y}_{N}$. More importantly, the smoothing technique can improve the estimation during the line of sight occlusion. Since it can estimate the orientation by integrating angular velocity both forwards and backwards in time during the occlusion.

Among all smoothers, Rauch-Tung-Striebel (RTS) smoother exploits fixedinterval Kalman smoothing technique [123, 124, 125] and is well known for its efficiency. Therefore, we chose this smoother for improving the estimation accuracy under offline condition. The implementation of RTS can be divided into two steps: The first step is to apply the ESKF described above and save the estimates for $\hat{\boldsymbol{q}}_{k}, \hat{\boldsymbol{b}}_{k}, \boldsymbol{x}_{\varepsilon, k}, \boldsymbol{x}_{\varepsilon, k}^{-}, \boldsymbol{P}_{\varepsilon, k}$ and $\boldsymbol{P}_{\varepsilon, k}^{-}$. The second step is to perform a backward EKF which computes the smoothing state vector with the parameter saved in the prior ESKF. The gain $\boldsymbol{A}_{\varepsilon, k}$ is calculated as:

$$
\boldsymbol{A}_{\varepsilon, k}=\boldsymbol{P}_{\varepsilon, k} \boldsymbol{F}_{\varepsilon, k}^{T}\left(\boldsymbol{P}_{\varepsilon, k+1}^{-}\right)^{-1}
$$


Subsequently, the state vector $\boldsymbol{x}_{\varepsilon, k}$ is updated based on $\boldsymbol{A}_{\varepsilon, k}$ :

$$
\boldsymbol{x}_{s \mid \varepsilon, k}=\boldsymbol{x}_{\varepsilon, k}+\boldsymbol{A}_{\varepsilon, k}\left(\boldsymbol{x}_{s \mid \varepsilon, k+1}-\boldsymbol{x}_{\varepsilon, k}^{-}\right)
$$

Finally, the covariance matrix $\boldsymbol{P}_{\varepsilon, k}$ is updated as:

$$
\boldsymbol{P}_{s \mid \varepsilon, k}=\boldsymbol{P}_{\varepsilon, k}+\boldsymbol{A}_{\varepsilon, k}\left(\boldsymbol{P}_{s \mid \varepsilon, k+1}-\boldsymbol{P}_{\varepsilon, k+1}^{-}\right)
$$

where $\boldsymbol{x}_{s \mid \varepsilon, k}$ and $\boldsymbol{P}_{s \mid \varepsilon, k}$ are estimated state vector and variance using the smoothing technique. The initial values are set as

$$
\boldsymbol{x}_{s \mid \varepsilon, N}=\boldsymbol{x}_{\varepsilon, N} ; \boldsymbol{P}_{s \mid \varepsilon, N}=\boldsymbol{P}_{\varepsilon, N}
$$

Based on Eq (4.19) and (4.23), the offset of the gyroscope $\hat{\boldsymbol{b}}_{k}$ is updated as:

$$
\hat{\boldsymbol{b}}_{k}=\hat{\boldsymbol{b}}_{k}^{-}-\boldsymbol{b}_{s \mid \varepsilon, k}
$$

The orientation $\hat{\boldsymbol{q}}_{k}$ is updated as:

$$
\hat{\boldsymbol{q}}_{k}=\hat{\boldsymbol{q}}_{k}^{-} \otimes\left[\begin{array}{ll}
1 & \frac{1}{2} \boldsymbol{\theta}_{s \mid \varepsilon, k}
\end{array}\right]
$$

\subsection{Experiments}

To validate the proposed algorithm, two experiments were performed. The first experiment was to evaluate the performance of the algorithm during $3 \mathrm{D}$ static and dynamic movements. The measurement unit including a cluster of markers and a gyroscope was placed statically and rotated in 3D space with yaw, pitch and roll rotations. The second experiment was to evaluate its performance during a biomedical application, being the estimation of the relative orientation between the hand and the index finger during a flexion and extension movement.

\subsubsection{Experiment setups}

We used 3D rate gyroscopes included in the MPU9250 (InveSense) in our experiment. The sampling frequency of the gyroscope was $200 \mathrm{~Hz}$. The data was transmitted to the PC through a USB connection. The Vicon system with 8 cameras, as shown in Fig 4.4, was chosen as the OTS system. The sampling frequency was $100 \mathrm{~Hz}$. 


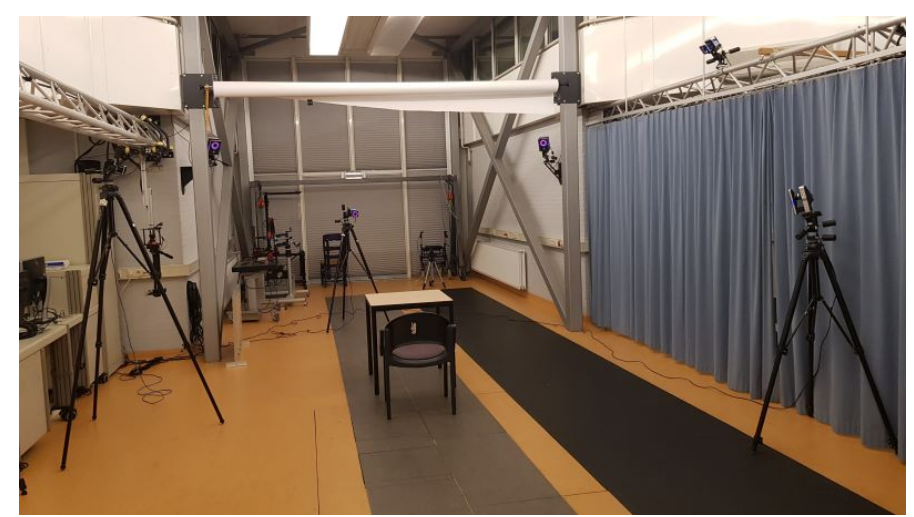

Figure 4.4: Vicon system with 8 cameras. A table in the middle of cameras was used a support during the static experiment. All the data was pre-processed with the Vicon Nexus 2.10.2 software.

In the first experiment, the cluster of markers formed an isosceles right triangle $p_{1} p_{2} p_{3}$, which is attached above the surface of the gyroscope, as shown in Fig 4.5. The distances between markers depends on applications. It will, for example, be different when comparing human leg tracking with fingertip tracking. In order to evaluate the performance of the proposed method for different distances between markers, the leg $r_{12}$ was set to three different values: $13 \mathrm{~mm}, 18 \mathrm{~mm}, 24 \mathrm{~mm}$. Another Cluster of markers form a second isosceles right triangle $P_{1} P_{2} P_{3}$. The leg $R_{12}$ was $100 \mathrm{~mm}$. The orientation estimate based on $P_{1} P_{2} P_{3}$ is much more accurate than the orientation estimate based on $p_{1} p_{2} p_{3}$, and was therefore used as an orientation reference.

In the second experiment, two measurement units were attached to the dorsal side of the hand and the index fingertip, as shown in Fig 4.6. No orientation reference was used in this experiment.

\subsubsection{Synchronization}

The gyroscope and OTS. Both systems were independent and sampled individually. Therefore, they needed to be synchronized before data fusion. We obtained an estimate of the modulus of the angular velocity from the OTS system in the following manner: First, we determined the quaternion-based orientation from the markers using Eq (4.4)

$$
\tilde{\boldsymbol{q}}_{g, k}^{m}=\left(\boldsymbol{q}_{m, k}^{g}\right)^{-1}
$$




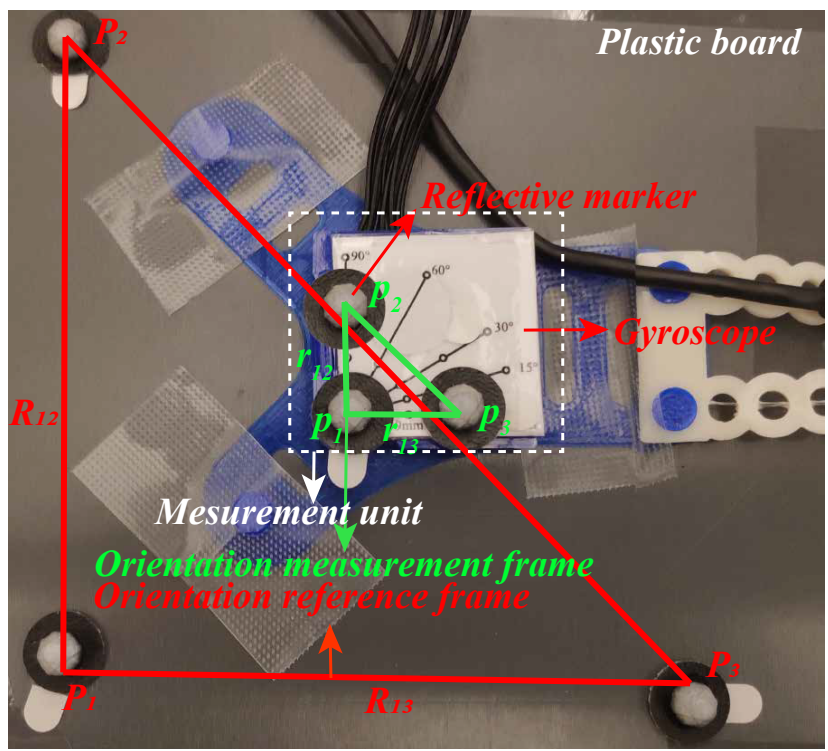

Figure 4.5: Markers and the gyroscope setup. One cluster of markers was attached on top of a gyroscope. It formed an isosceles right triangle $p_{1} p_{2} p_{3}$. The other cluster of markers form an isosceles right triangle $P_{1} P_{2} P_{3}$. The leg $R_{12}$ of the second marker configuration was much longer than $r_{12}$ of the first configuration. Thus, the orientation from $P_{1} P_{2} P_{3}$ is more accurate and was therefore used as an orientation reference. The length of $r_{12}$ was varied, the length of $R_{12}$ was approximately $100 \mathrm{~mm}$.

Using Eq (4.9), we derived

$$
\delta \tilde{\boldsymbol{q}}_{g, k}^{m}=\left(\tilde{\boldsymbol{q}}_{g, k}^{m}\right)^{-1} \otimes \tilde{\boldsymbol{q}}_{g, k+1}^{m}
$$

Subsequently, we estimated the angular velocity $\tilde{\boldsymbol{\omega}}_{k}^{m}$ using the following relation

$$
\delta \tilde{\boldsymbol{q}}_{g, k}^{m} \approx\left[\begin{array}{cc}
1 & \frac{1}{2} d_{t} \tilde{\boldsymbol{\omega}}_{k}^{m}
\end{array}\right]
$$

From the gyroscope, we obtained the measured angular velocity $\boldsymbol{y}_{g y r, k}^{s}$. Using Eq (4.8), we estimate $\tilde{\boldsymbol{\omega}}_{k}^{m}$ from $\boldsymbol{y}_{g y r, k}^{s}$, taking into account offset $\boldsymbol{b}$, noise $\boldsymbol{e}^{m}$ and sensor to maker frame rotation $\boldsymbol{q}_{s, k}^{m}$. However, during the rotation, $\boldsymbol{b}$ and noise $\boldsymbol{e}^{m}$ are much smaller than $\boldsymbol{y}_{\text {gyr, }, k}^{s}$. The moduli of angular velocities from the OTS and the gyroscope are therefore assumed to be approximately the same, that is

$$
\left(\tilde{\boldsymbol{\omega}}_{k}^{m}\right)^{T} \tilde{\boldsymbol{\omega}}_{k}^{m} \approx\left(\boldsymbol{y}_{g y r, k}^{s}\right)^{T} \boldsymbol{y}_{g y r, k}^{s}
$$




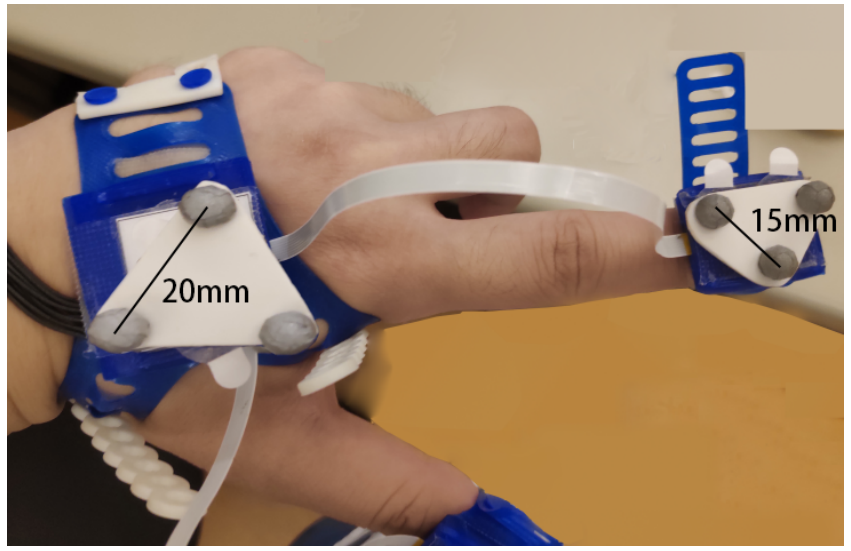

Figure 4.6: Measurement units used to estimate relative orientation between hand and index finger. Every measurement unit includes a gyroscope with a cluster of markers attached. One unit was attached to the dorsal side of the hand, the other to the tip of the index finger. The shorter legs of the isosceles right triangle formed by markers were $20 \mathrm{~mm}$ and $15 \mathrm{~mm}$ on dorsal side of the hand and index finger respectively.

The synchronization was done by maximizing the correlation between $\left|\tilde{\boldsymbol{\omega}}_{k}^{m}\right|$ and $\left|\boldsymbol{y}_{g y r, k}^{s}\right|$.

\subsubsection{Alignment}

Alignment between markers and the gyroscope in both experiments: In addition to synchronization, the alignment between the gyroscope and optical markers is vital for data fusion, since it is required for by comparing the angular velocity calculated by the markers and measured by the gyroscope.

According to Eq (4.8), $\boldsymbol{q}_{s}^{m}$ can be obtained with:

$$
\operatorname{argmin}_{\boldsymbol{q}_{s}^{m}} \quad\left\|\overline{\boldsymbol{\omega}}^{m}-\boldsymbol{q}_{s}^{m} \otimes\left(\overline{\boldsymbol{y}}_{g y r}^{s}-\boldsymbol{b}^{s}\right)\right\|_{2}^{2}
$$

The result of alignment between the gyroscope and OTS are described in Appendix A.2.

Alignment between two clusters of markers in experiment 1: Before the cluster $P_{1} P_{2} P_{3}$ can be used as reference for $p_{1} p_{2} p_{3}$, it is essential to align them. The relative orientation between the two clusters can be estimated from the orientation of each cluster, with respect to the global frame according to eq (4.4) when the object with both marker clusters is held in a static 
posture. The error angle $\boldsymbol{\theta}_{\text {err }}$ showed in Fig 4.7, 4.8 is determined in Eq (4.6) and (4.7).

\subsubsection{Experiment procedures}

\section{Experiment 1- Orientation estimation in 3D space}

Two cluster markers and an inertial measurement unit (MPU925) were attached to a rigid plate, as shown in Fig 4.5. The plate was hold still for $60 \mathrm{~s}$ in one orientation and was subsequently rotated randomly in 3D space for $60 \mathrm{~s}$. Subsequently, the length of $r_{12}$ was changed from $13 \mathrm{~mm}$ to $17 \mathrm{~mm}$ and $24 \mathrm{~mm}$, and the static and dynamic movements were repeated.

The setup of markers and cameras was designed such that all markers were always visible by enough cameras. The line of sight occlusion was implemented in the analysis by leaving out the position of one or several of the markers of cluster $p_{1} p_{2} p_{3}$ during a certain time period. During the lineof-sight occlusion, the positions of the reference set $P_{1} P_{2} P_{3}$ were visible, and therefore the reference orientation, was available.

\section{Experiment 2- Relative orientation estimation between hand and fingers}

Two marker clusters and gyroscopes were attached on the dorsal side of the hand and index fingertip, as shown in Fig 4.6. First, the sensor to segment calibration was performed, including the sensor to hand and the sensor to index fingertip calibration:

1. The hand was placed on a horizontally flat surface, the back of the palm pointing upwards;

2. The hand was placed against a vertically flat surface;

3. The hand was raised and the index finger was repeatedly flexed and extended.

After the above procedure, the sensor to segment calibration was done by exploiting that a static accelerometer only measures gravitational acceleration and a gyroscope measures angular velocity. More details about the sensor to segment calibration can be found in [102].

Finally, flexion and extension movements were performed with the index finger, approximately once per second, while not rotating nor displacing the hand. The rotation angle for index finger was approximately $150^{\circ}$. The 
orientation of the whole hand was subsequently changed and the flexion and extension movements repeated.

\subsection{Results}

\subsubsection{Experiment 1- Orientation estimation in 3D space}

Static performance: The results of the static trials are shown in Fig 4.7. Subfigure (a), (b) and (c) show the marker distances $r_{12}$, while (d) to (f) show the corresponding orientation errors, and (h) to (j) are the corresponding error distributions in (d) to (f). When marker distance $r_{12}$ increasedr, the corresponding orientation estimation error became smaller. Initial peaks in Fig 4.7d, 4.7e and 4.7f are the result of "switch-on transient behavior" due to the initial value of the error covariance matrix $\boldsymbol{P}_{\varepsilon, k}$. When applying the ESKF separately or in combination with the smoothing method, the estimation errors reduced extensively. The estimation errors when the ESKF was applied separately or in combination with the smoother did not differ significantly. The detailed orientation errors are specified in Table I.

Dynamic performance: The results during dynamic trials are shown in Fig 4.8. These results demonstrate two advantages of the proposed fusion method when compared with applying only the OTS: both accuracy and orientation estimation during line of sight occlusion improved.

Accuracy improvement: Subfigure (a) to (c) of Fig 4.8 show the measured marker distances $r_{12}$ for the three marker configurations that were evaluated, while (d) to (f) show the corresponding orientation errors, and (h) to (j) are the corresponding error distributions under the condition that no lineof-sight occlusion was implemented. When marker distance $r_{12}$ increased, the corresponding orientation error appeared to become smaller. Furthermore, the orientation error were smaller after fusion with the gyroscope using an ESKF and additional smoothing. The smoothing technique improved the result of ESKF. The detailed values are specified in Table I.

Data filling during line-of-sight occlusion: The optical data between 20s and 40s was assigned as unavailable (Fig 4.9). During this period, the orientation was filled with gyroscope data automatically. As shown in Fig 4.9 , The orientation error increased to $4.8^{\circ}$ in 20 s by only exploiting the gyroscope, which was reduced to $2.5^{\circ}$ by additionally using the smoothing technique. 

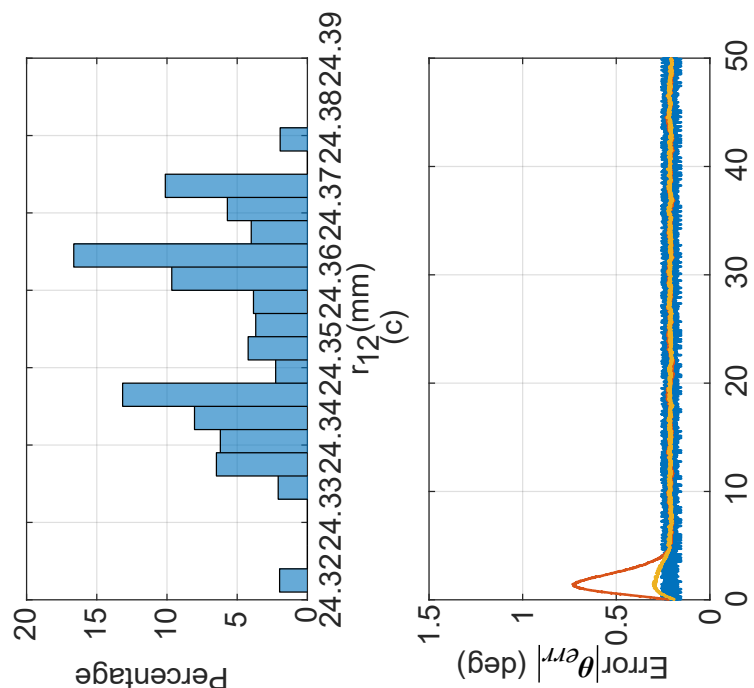

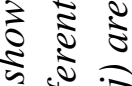

(4) $: 0$

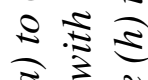
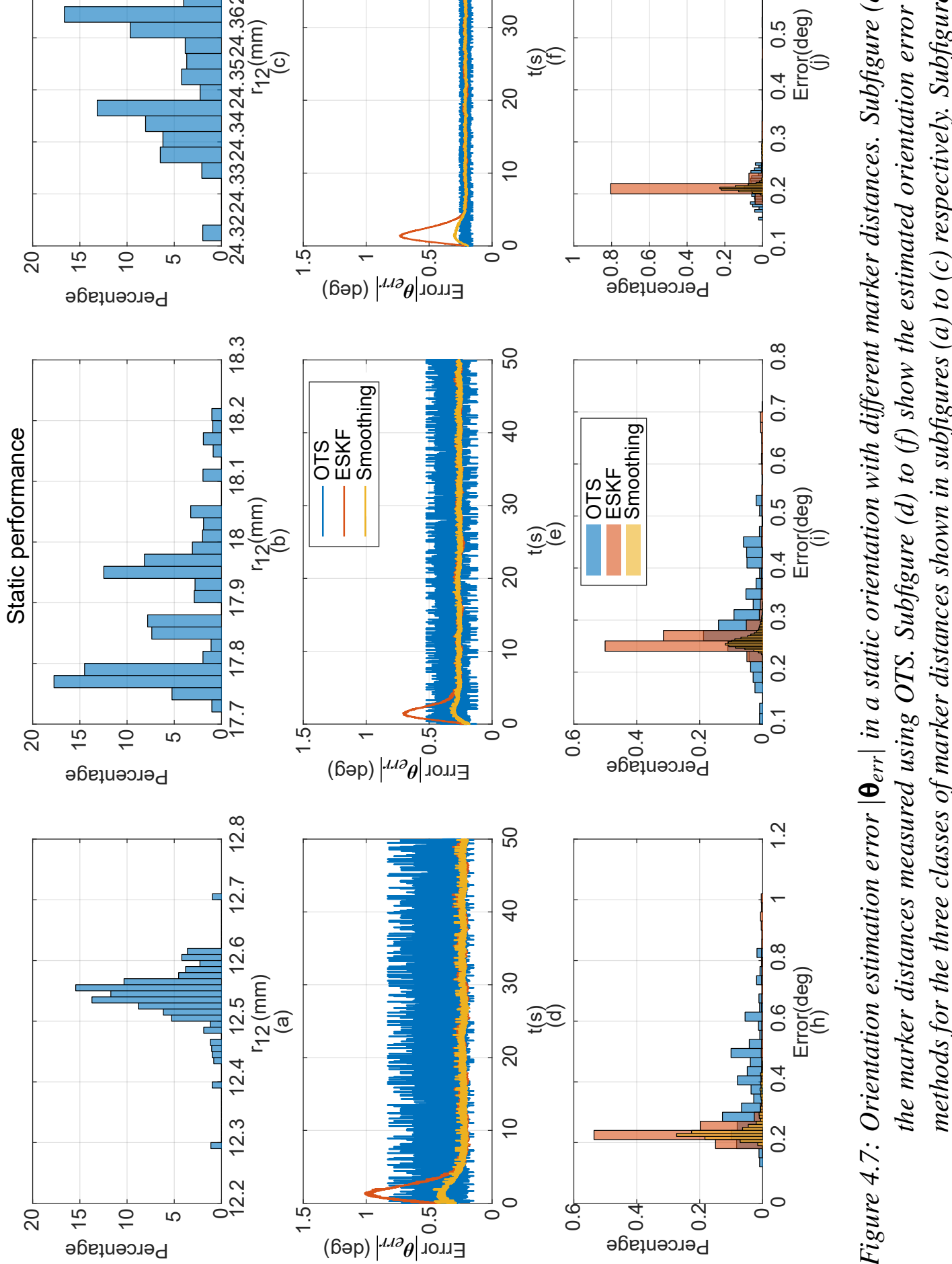

$\searrow$
$\forall$
0
$\vdots$
0
0 

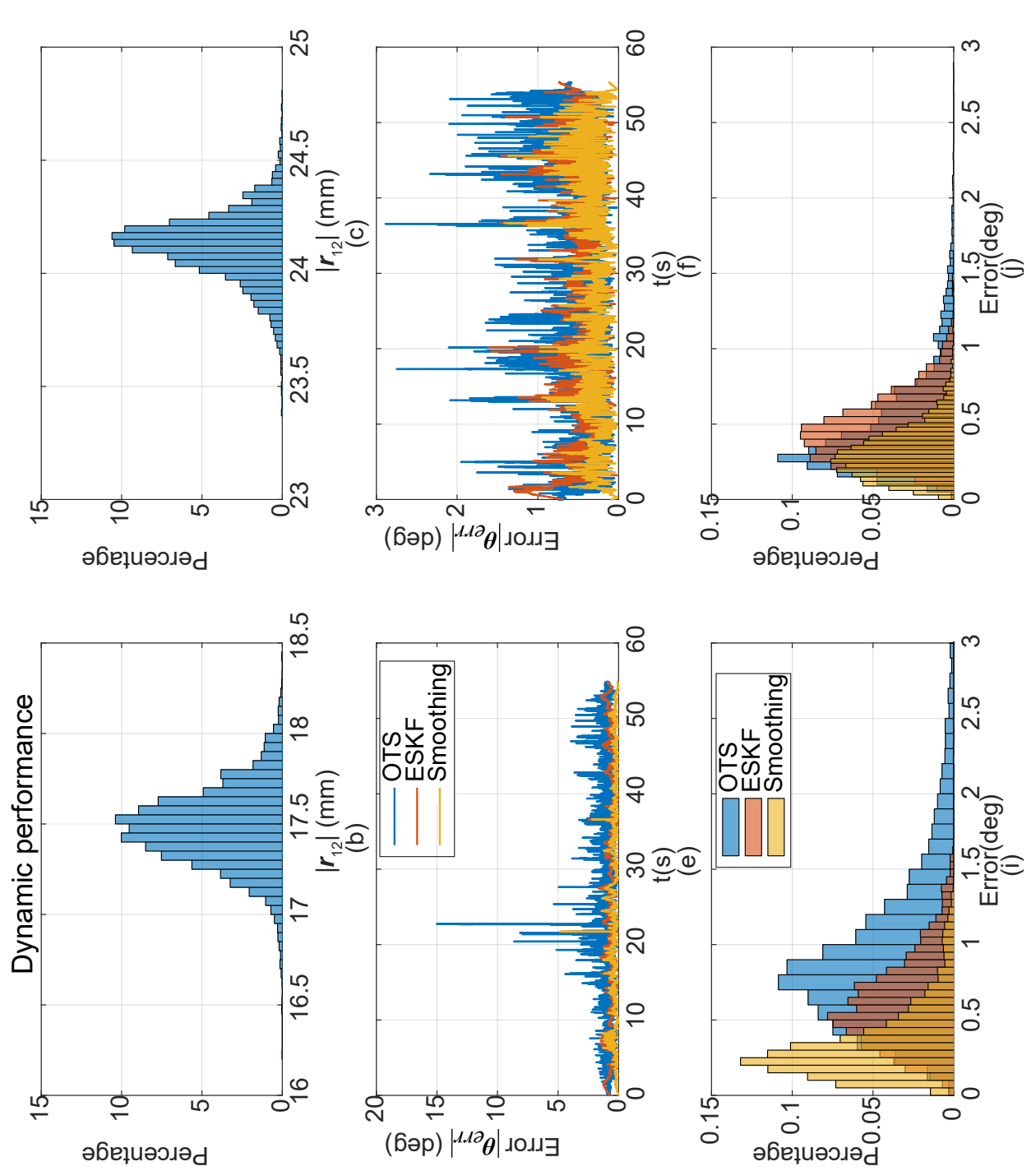

ㄴำ

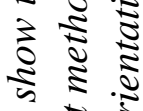

ปิ

$\therefore \underbrace{2} \frac{1}{2}$

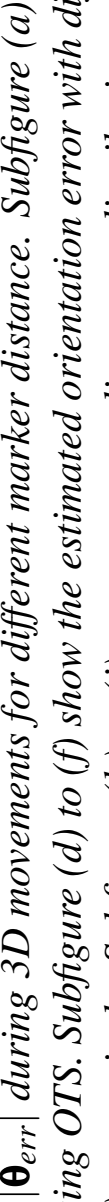

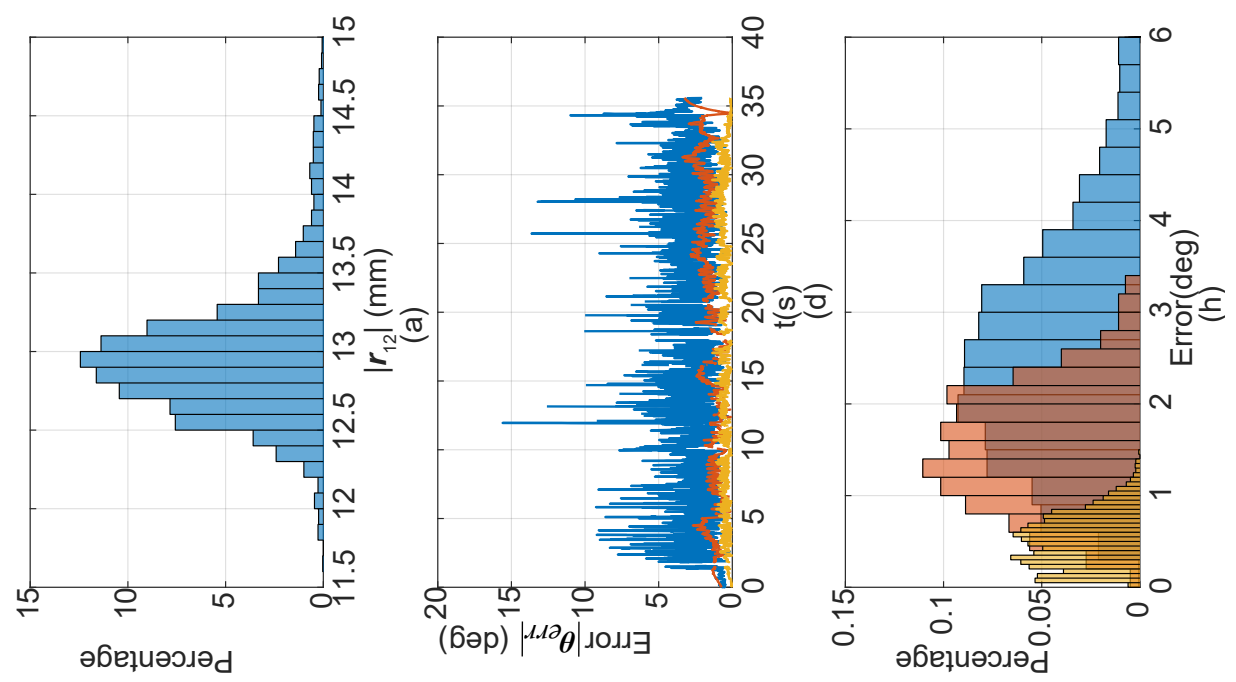

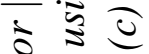

$2 \sqrt{2}$

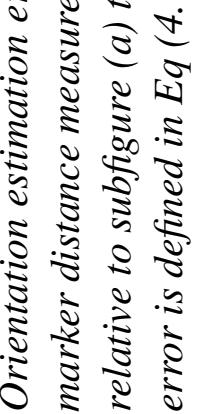

$\ddot{\infty}$

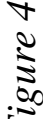


Table 4.1: Orientation error with OTS and ESKF for different marker distances

\begin{tabular}{ccccc}
\hline Items & & $r_{12}=13 \mathrm{~mm}$ & $r_{12}=18 \mathrm{~mm}$ & $r_{12}=24 \mathrm{~mm}$ \\
\hline \multirow{2}{*}{ OTS (deg) } & static & $0.39 \pm 0.16$ & $0.31 \pm 0.08$ & $0.21 \pm 0.03$ \\
& dynamic & $2.75 \pm 1.56$ & $0.96 \pm 0.74$ & $0.49 \pm 0.34$ \\
ESKF (deg) & static & $0.23 \pm 0.02$ & $0.26 \pm 0.01$ & $0.21 \pm 0.00$ \\
& dynamic & $1.50 \pm 0.62$ & $0.54 \pm 0.26$ & $0.43 \pm 0.20$ \\
Smoothing (deg) & static & $0.23 \pm 0.02$ & $0.26 \pm 0.01$ & $0.21 \pm 0.00$ \\
& dynamic & $0.54 \pm 0.28$ & $0.36 \pm 0.25$ & $0.31 \pm 0.17$ \\
\hline
\end{tabular}

${ }^{*} r_{12}$ represents the distance between markers, refer to Fig 4.5. $r_{12}$ is obtained from OTS under static condition. $r_{12}$ and $r_{13}$ are approximately equal, $\left|r_{12}-r_{13}\right| \leq 2 \mathrm{~mm}$.

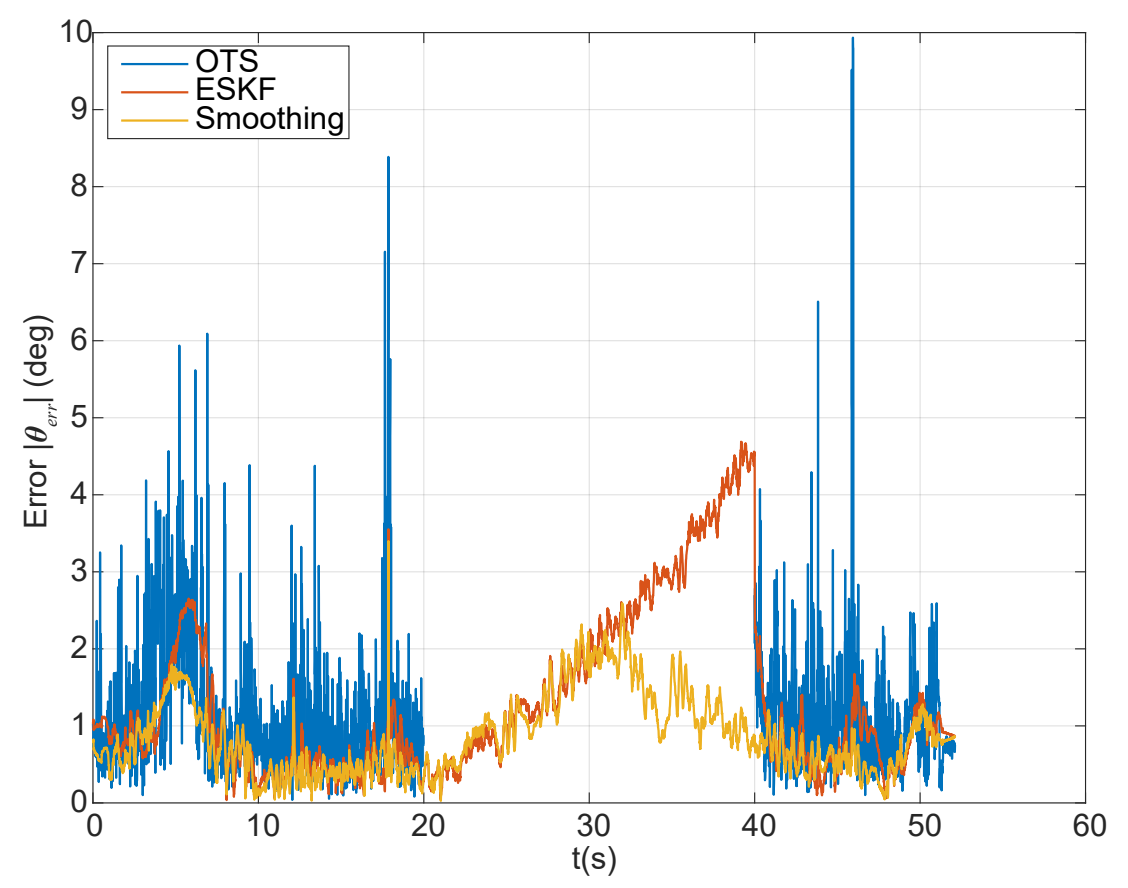

Figure 4.9: Orientation estimation error with OTS separately and with EKSF fusion of OTS and gyroscope measurements, with and without additional smoothing (indicated by EKS and smoothing). The orientation error is defined in Eq (4.6) and (4.7). During the measurement, pitch, roll and yaw movements were performed. 
4 Improvement of optical tracking-based orientation

\subsubsection{Experiment 2-Relative orientation estimation between hand and fingers}

The results were divided into three cases: Case I-No line-of sight occlusion; Case II-With an occlusion of markers; Case III- With a wrong identification of markers by the OTS system. The angle $\beta$ shown in Fig 4.10-4.13 was determined by the relative orientation between hand and fingers $\boldsymbol{q}_{k}$, expressed in quaternions.

$$
\boldsymbol{q}_{k}=\left[\begin{array}{ll}
\cos \frac{\beta_{k}}{2} & \sin \frac{\beta_{k}}{2} \boldsymbol{n}_{k}
\end{array}\right]
$$

where $\beta_{k}$ is rotation angle, $\boldsymbol{n}_{k}$ is the direction of the rotation axis.

\section{Case I-No line-of sight occlusion}

During one trial of flexion and extension movements, markers were observable and identified correctly. The results are shown in Fig 4.10.

Fig 4.10a represents the flexion/extension angle $\beta$, estimated by fusing OTS and gyro information using an ESKF, and applying subsquent smoothing. The estimation based on ESKF and smoothing are smoother than the estimation based on only OTS, as shown in (b) of Fig 4.10. (b) is an enlarged part of (a) between 10.2s and 10.9s.

During one trial of flexion and extension of the index finger, markers were not well observed, resulting in line of sight occlusions and marker identification problems. The results are shown in Fig 4.11.

\section{Case II-With an occlusion of markers}

Fig 4.12 is an enlarged part of Fig 4.11 during 2.7 3.4s. Orientation estimation based on ESKF and smoothing appeared to be well able to fill the gap during the line of sight occlusion.

\section{Case III- With a wrong identification of markers by the OTS system}

The OTS constantly identifies all markers during a measurement. A frequent error concerns an erroneous marker identification, for example after markers cross each other in one or several of the camera vies. This can result in sudden jumps of OTS-based 3D orientation estimates. Fig 4.13 is an enlarged part of Fig 4.11 during 1.1 1.5s. In this case, markers were observed but not correctly identified. With the OTS, relative orientation $\boldsymbol{q}_{k}$ was 
4.4.2 Experiment 2-Relative orientation estimation between hand and fingers
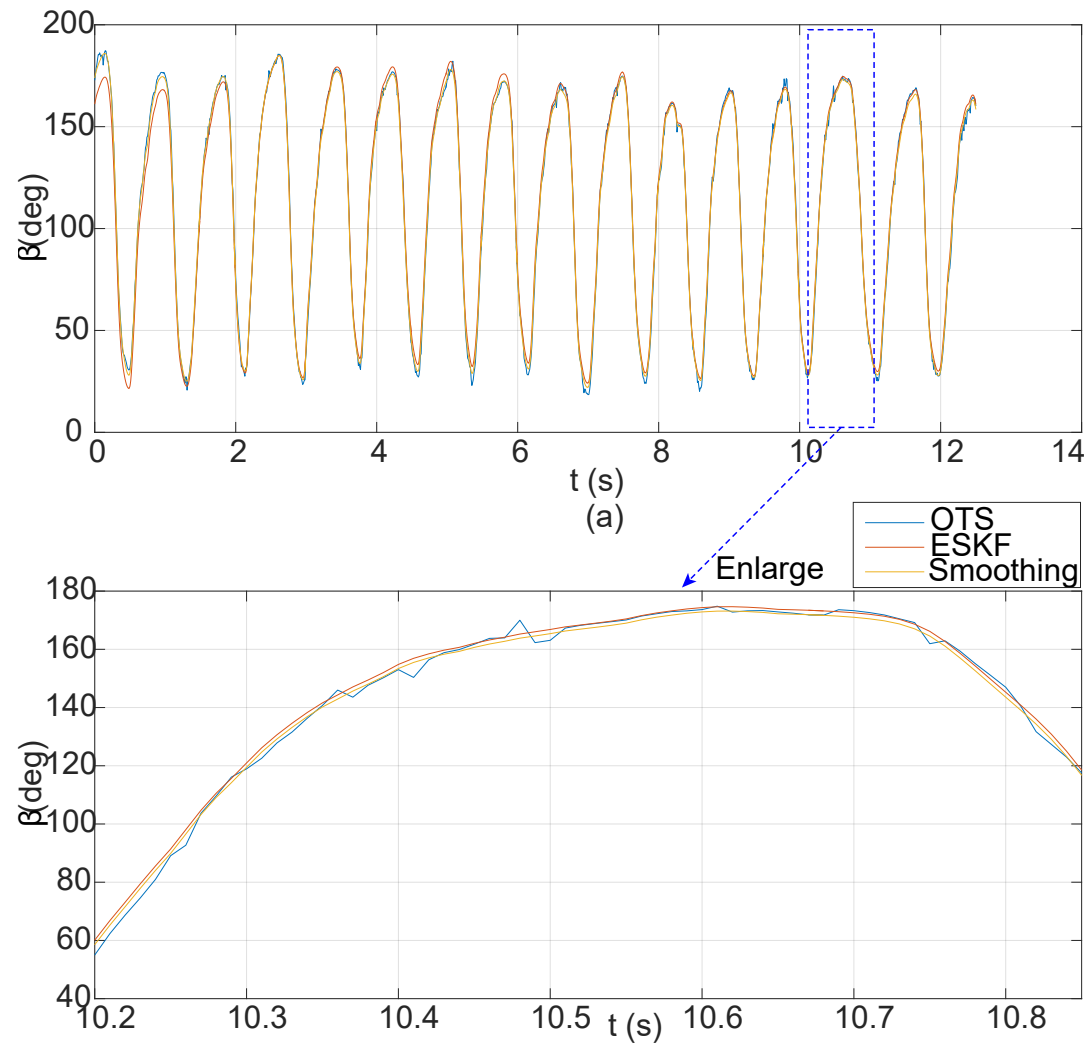

(b)

Figure 4.10: Estimation of rotation angle $\beta$ during flexion and extension movements of the index finger. $\beta$ is determined by Eq (4.54). During the movement, all the markers were observable and identified correctly. Subfigure (a) is the results during the whole movement. (b) is an enlarged part of (a) between $10.2 \mathrm{~s}$ and $10.9 s$.

estimated as $-\boldsymbol{q}_{k}$, therefore $\beta$ was estimated as $304.7^{\circ}$ instead of $55.4^{\circ}$. In comparison, the ESKF and the smoothing method can provide a good estimate after fusing with gyroscope information. 


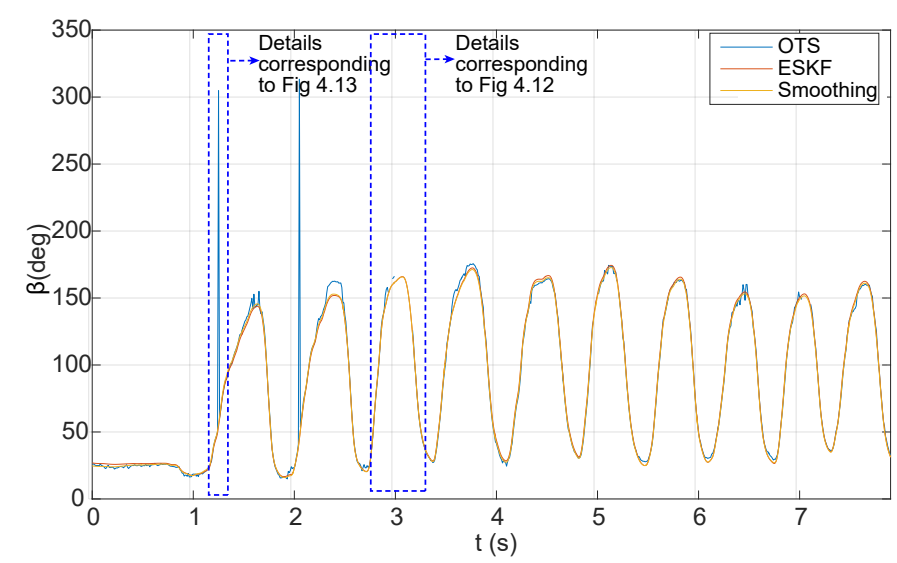

Figure 4.11: Estimation of rotation angle $\beta$ during flexion and extension movements. In this trial, line of sight occlusion occurred around $3 s$ and a marker identification problem between $1.1 \sim 1.5 s$.

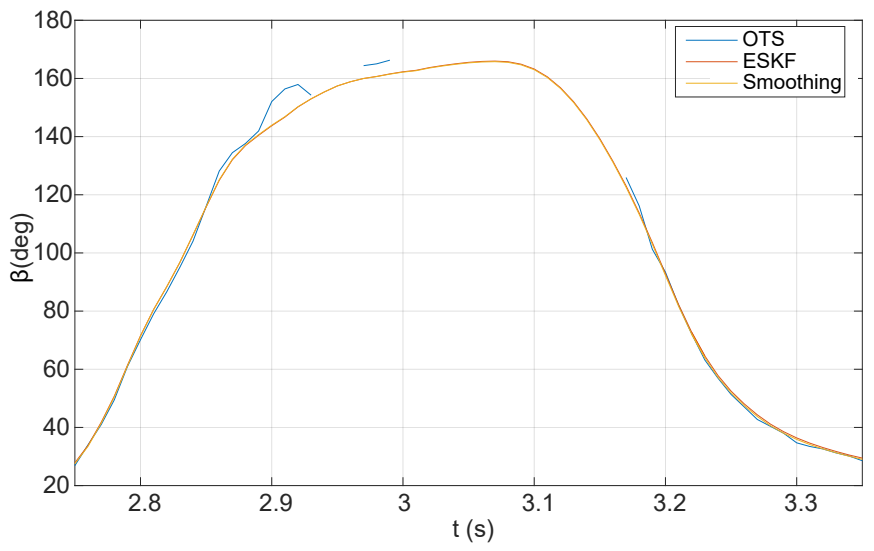

Figure 4.12: Estimation of rotation angle $\beta$ during the line of sight occlusion (indicated by gaps in the blue line). This is an enlarged part of Fig 4.11 during 2.7 3.4s.

\subsection{Discussion}

The accuracy of orientation estimation based on the OTS depends on the distance between markers. According to Wiles et al. [126], the orientation error is about $0.4^{\circ}$ with the Polaris system, Canada. The shape of the marker cluster was an isosceles right triangle and the length of the leg was $50 \mathrm{~mm}$. 


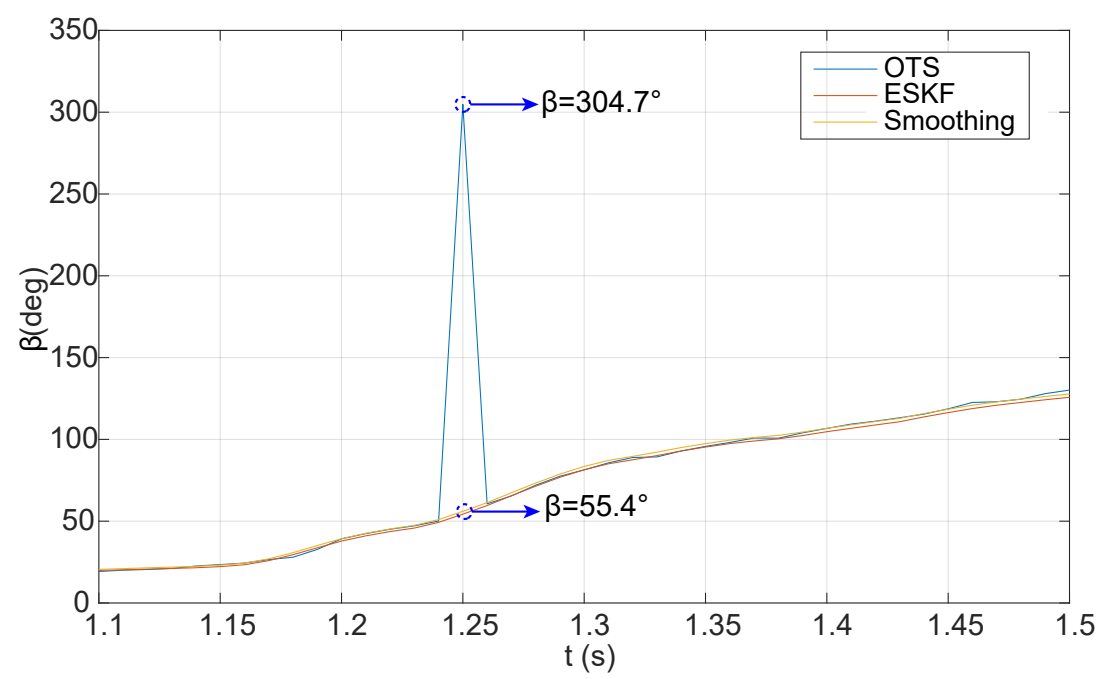

Figure 4.13: Estimation of rotation angle $\beta$ in the case of a marker identification problem. This is an enlarged part of Fig 4.11 at $1.25 \mathrm{~s}$.

In our current study, the mean error changed from $2.8^{\circ}$ to $0.5^{\circ}$ when the distance between markers changed from $13 \mathrm{~mm}$ to $24 \mathrm{~mm}$. After fusing with gyroscope data, the mean error reduced to $1.5^{\circ}$ and $0.4^{\circ}$ with the ESKF. Compared with the results of Wiles et al., we achieved a comparable performance when fusing OTS and gyroscope information at half the distance between markers. This is quite useful for the applications that require high accuracy but with limited space to mount markers, such as finger segments tracking.

In previous research of $\mathrm{He}$ et al. [27], gyroscope, accelerometer and magnetometer were used to improve the position and orientation accuracy of OTS. The algorithm is shown in subfigure (a) of Fig 4.14. They used position measurements from OTS and orientation estimates from IMU and magnetometer in the measurement model to update the position and orientation estimate. However, including accelerometer and magnetometer for orientation estimation is not optimal since the inclination estimate derived from the accelerometer is disturbed by non-gravity acceleration, and the heading estimate derived from magnetometer is disturbed by ferromagnetic materials and magnetic sources in the environment. By fusing with OTS position measurements, errors from the accelerometer and magnetometer can be partially reduced but cannot be eliminated. As shown in (b) of Fig 4.14, our method does not involve an accelerometer or magnetometer. Therefore, ori- 
entation estimation errors caused by non-gravity acceleration and magnetic disturbances are not present in our estimates. The estimated performances with different methods are shown in Fig 4.15, the data is the same as the subfigure (e) of Fig 4.8. During the static period $0 \sim 10$ s, the performances of He et al.'s method, the ESKF method and the smoothing method are similar. During the dynamical period $10 \sim 64 \mathrm{~s}$, the estimation based on He et al.'s method has a larger error than our ESKF and the smoothing method. As shown in Fig 4.16, the non-gravitational acceleration during 10.1 20.2s caused a large orientation error, which can be clearly seen in Fig 4.15.
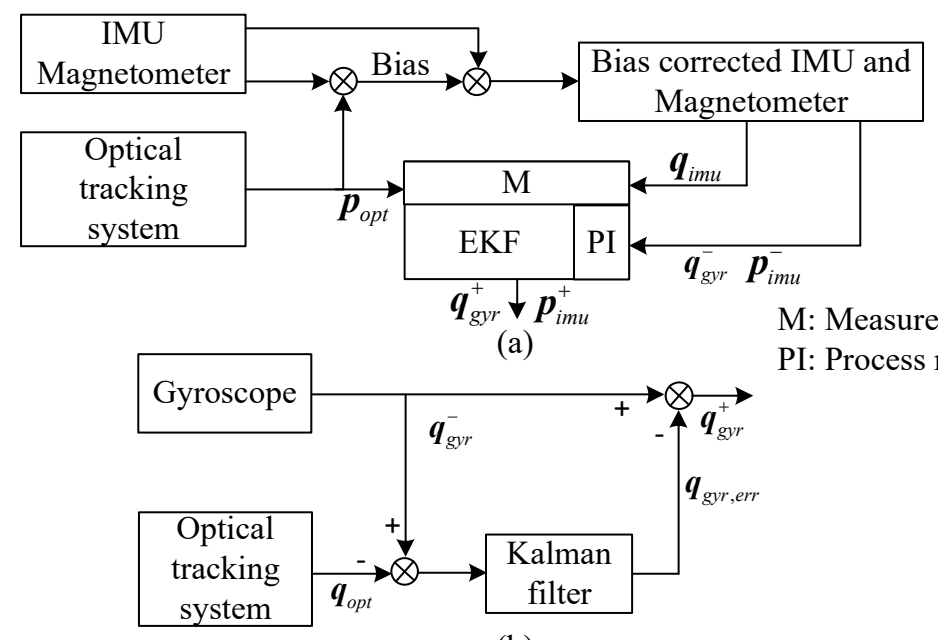

M: Measurement model PI: Process model

(b)

Figure 4.14: Two algorithms to estimate 3D orientation. Subfigure (a) represents the fusion of OTS with IMU based on He et al.'s research [27]. Orientation estimates are first obtained from IMU and magnetometer, and subsequently fused with OTS measurements. The initial orientation estimate is disturbed by nongravity acceleration and magnetic disturbances. Subfigure (b) shows our fusion method with OTS and gyroscope. It does not rely on magnetometer and accelerometer measurements.

The RTS smoother described in page 5 is a supplement to the proposed ESKF method, not a mandatory step. It is chosen for its efficiency and ease of implementation. Other smoothers such as Modified Bryson-Frazier smoother, Minimum-variance smoother etc [127, 128] can also be applied. The limitation of RTS smoother is that it needs all the measurement data. In order to compromise between real-time use and accuracy, a smoother that 


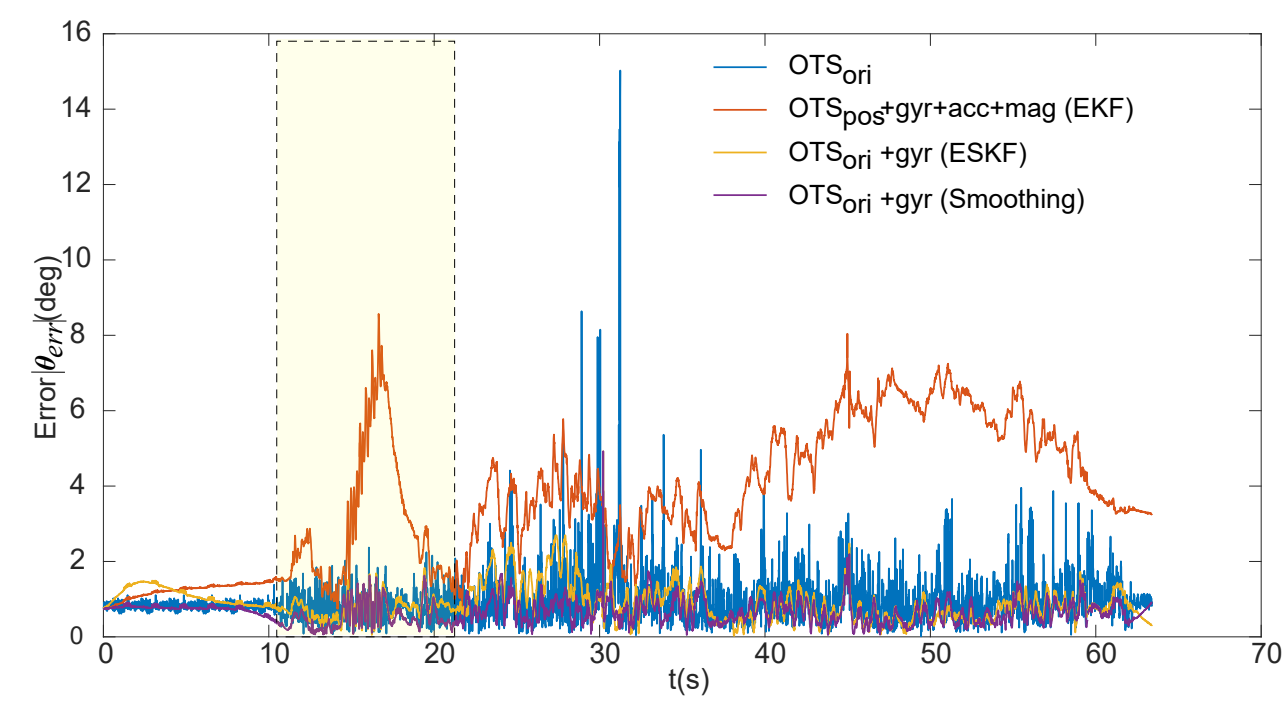

Figure 4.15: Comparison of orientation estimations results with different methods. During the tests, 3D pitch, roll and yaw rotations were performed. The brown line represents the results with the method described by He et al., which relies on the position estimation from OTS, gyroscope, accelerometer and magnetometer. The blue line represents the result based on orientation from OTS. The yellow line represents the result based on orientation from OTS and gyroscope.

only exploits the data within a window can be designed.

Our method does not solve the orientation estimation errors during a long line-of-sight occlusion. However, it can help fill short occlusion gaps in the order of $10 \sim 20 \mathrm{~s}$. It should be noted that occlusion problems usually last for a few seconds. Therefore, our fusion methods is a good option to improve estimation accuracy and reduce orientation estimation drift to acceptable levels during relatively short occlusion. The error can even be reduced if offline smoothing is applied (see Fig. 4.9). The fusion with accelerometer and magnetometer during long occlusions may improve the accuracy since they provide disturbed orientation references in addition to only integration of angular velocity by the gyroscope.

ESKF was applied to fuse OTS and gyroscope information since it was reported to have a better performance in some areas, such as IMMU-based human movement tracking [40]. However, small drawbacks still exist. Com- 


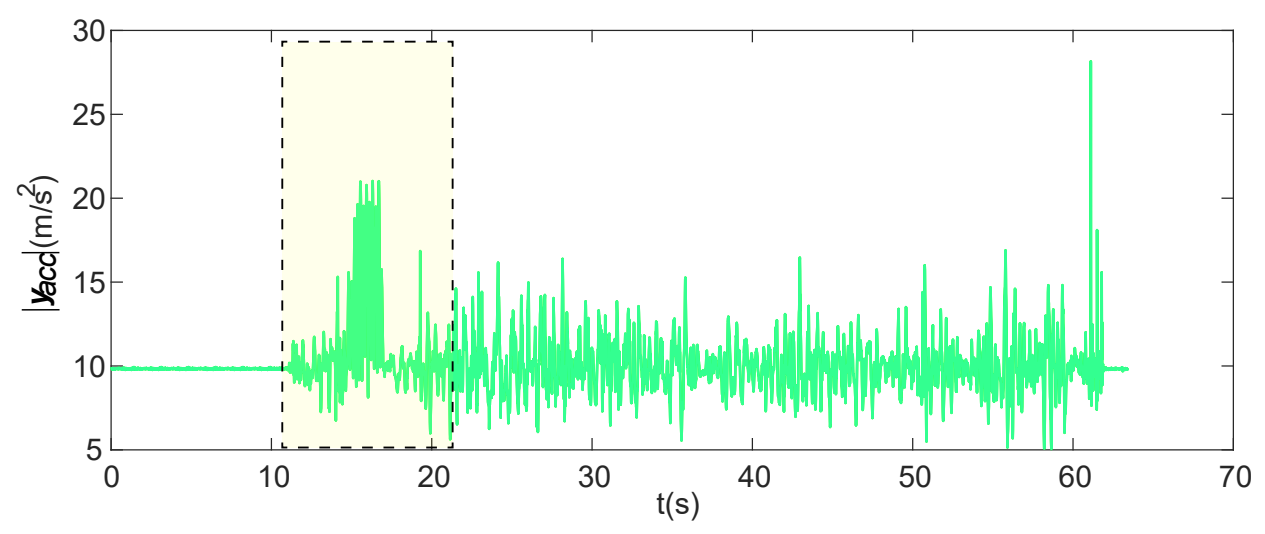

Figure 4.16: Modulus of the accelerometer outputs during the movements presented in Fig 4.15. During 10.1 20.2s, there was a rapid rotation which caused a large non-gravitational acceleration, the corresponding orientation estimation is shown in the shadow part of Fig 4.15.

pared with the EKF, ESKF has a more complex structure since two states need to be updated: nominal state and error state. In addition, the computational cost is higher.

\subsection{Conclusion}

An ESKF approach with an optional RTS smoother was proposed in this paper to fuse orientation estimates from OTS and rate gyroscope. The objective of this approach was to improve the 3D orientation estimation of small human body segments, like finger tips. When the proposed method was applied to a single unit of three OTS markers connected to a rate gyroscope moving in 3D space. Two improvements were achieved. Firstly, the accuracy of OTS-based orientation estimation was improved when markers were observable. Statically, the OTS-based orientation errors were $0.39^{\circ} \pm 0.16^{\circ}$ and $0.21^{\circ} \pm 0.03^{\circ}$ when the distances between markers were $13 \mathrm{~mm}$ and $24 \mathrm{~mm}$. These errors were reduced to $0.23^{\circ} \pm 0.02^{\circ}$ and $0.21^{\circ} \pm 0.00^{\circ}$ when applying ESKF and smoothing. under dynamic conditions, in which the orientation was continuously changed, the OTS-based orientation errors were $2.75^{\circ} \pm 1.56^{\circ}$ and $0.49^{\circ} \pm 0.34^{\circ}$ when the distances between markers were $13 \mathrm{~mm}$ and $24 \mathrm{~mm}$. This reduced to $1.50^{\circ} \pm 0.62^{\circ}$ and $0.43^{\circ} \pm 0.20^{\circ}$ with the 
ESKF, $0.54^{\circ} \pm 0.28^{\circ}$ and $0.31^{\circ} \pm 0.17^{\circ}$ with the additional use of the smoother. Secondly, ESKF and RTS smoother were able to fill gaps in OTS orientation estimates during line-of-sight occlusion. The orientation error increased to $4.8^{\circ}$ during an occlusion of 20 s with the ESKF, which was reduced to $2.5^{\circ}$ with the RTS smoother. When the proposed method was applied to relative orientation estimation between hand and fingers, we demonstrated three advantages: firstly, it smoothed the orientation estimates when OTS estimation had large perturbations. Secondly, it filled the orientation during an occlusion period. Finally, it corrected the relative orientation estimates when the OTS wrongly identified markers.

\section{Appendix A}

\section{A.1 Error angle $\left|\boldsymbol{\theta}_{e r r}\right|\left(\right.$ deg) with different $\sigma_{m}$ and $\sigma_{m}$}

For the Monte Carlo analysis corresponding to Fig 4.2, the detailed value for Error angle $\left|\boldsymbol{\theta}_{\text {err }}\right|$ (deg) with different $\kappa$ and $\sigma_{m}$ are specified in Table 2.

Table 2: Mean value of error angle $\left|\boldsymbol{\theta}_{\text {err }}\right|$ (deg) with different $\kappa$ and $\alpha$

\begin{tabular}{ccccccccc}
\hline \multirow{2}{*}{ Items } & \multicolumn{8}{c}{$\sigma_{m}(\mathrm{~mm})$} \\
\cline { 2 - 9 } & 0.02 & 0.1 & 0.18 & 0.26 & 0.34 & 0.42 & 0.5 & 0.58 \\
\hline$\alpha=15^{\circ}$ & 0.26 & 1.32 & 2.37 & 3.42 & 4.50 & 5.55 & 6.63 & 7.71 \\
$\alpha=30^{\circ}$ & 0.15 & 0.75 & 1.34 & 1.94 & 2.54 & 3.13 & 3.74 & 4.33 \\
$\alpha=45^{\circ}$ & 0.12 & 0.57 & 1.03 & 1.49 & 1.95 & 2.41 & 2.87 & 3.34 \\
$\alpha=60^{\circ}$ & 0.10 & 0.50 & 0.90 & 1.30 & 1.70 & 2.10 & 2.5 & 2.9 \\
$\alpha=75^{\circ}$ & 0.09 & 0.47 & 0.84 & 1.21 & 1.59 & 1.96 & 2.34 & 2.71 \\
$\alpha=90^{\circ}$ & 0.09 & 0.46 & 0.82 & 1.19 & 1.56 & 1.92 & 2.28 & 2.65 \\
\hline
\end{tabular}

\section{A.2 Alignment}

Fig 17, (a), (b), (c) show the angular velocity from the gyroscope and OTS before alignment. (d), (e), (f) show the angular velocity from the gyroscope and OTS after alignment. The angular velocity difference is

$\left[\begin{array}{lll}4.4 \pm 2.9 & 3.9 \pm 2.6 & 0.2 \pm 0.1\end{array}\right] \mathrm{rad} / \mathrm{s}$ before the alignment. After the 
alignment, the difference is $\left[\begin{array}{lll}0.3 \pm 0.3 & 0.3 \pm 0.4 & 0.1 \pm 0.1\end{array}\right] \mathrm{rad} / \mathrm{s}$. The figure shows that the gyroscope and OTS estimates correspond well after the alignment.

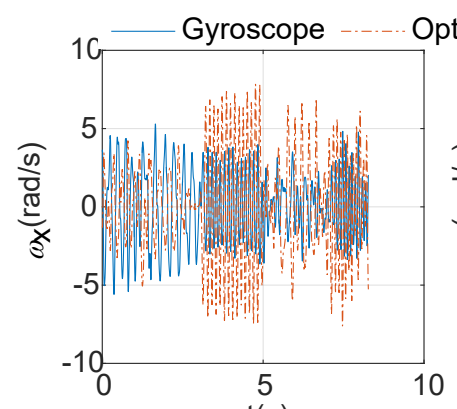

t(s)

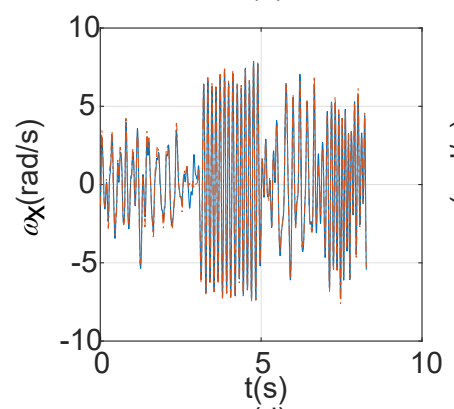

(d)

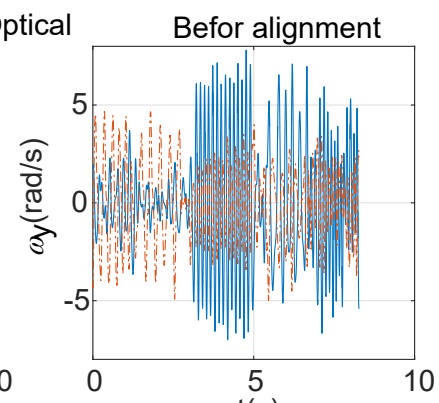

t(s)

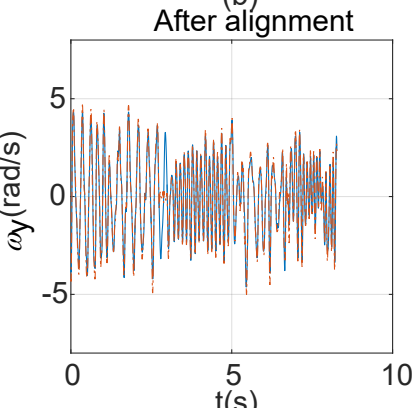

(e)

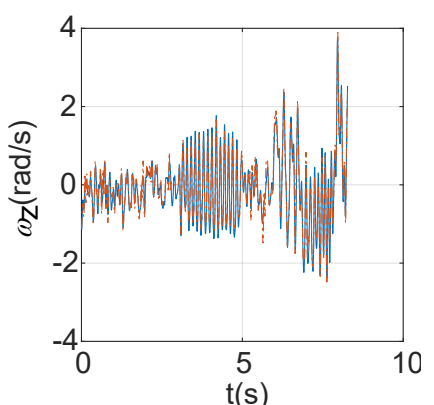

(c)

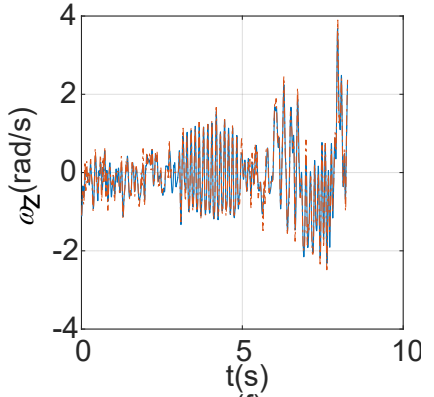

(f)

Figure 17: Alignment results based on angular velocity. The blue lines are angular velocities measured by gyroscope, the brown lines are angular velocity estimated from OTS measurements of positions of an optical marker cluster. Subfigure (a), (b) and (c) are angular velocities before alignment, subfigure $(d),(e)$ and $(f)$ are angular velocities after alignment. The angular velocities from gyroscope and optical system were both filtered with equiripple lowpass filter, the passband and stopband frequency were set as $8 \mathrm{~Hz}$ and $15 \mathrm{~Hz}$ respectively.

\section{A.3 Parameters for ESKF}

For the process model, we can differentiate with respective to error-state 
$\boldsymbol{\theta}_{\varepsilon, k-1}, \boldsymbol{b}_{\varepsilon, k-1}$ based on Eq (4.26)

$$
\begin{gathered}
\frac{\partial\left(\boldsymbol{\theta}_{\varepsilon, k}\right)}{\partial\left(\boldsymbol{\theta}_{\varepsilon, k-1}\right)}=\left(\boldsymbol{I}_{3 \times 3}-\left\lfloor\hat{\boldsymbol{\omega}}_{k-1}^{m}\right\rfloor_{\times} d_{t}\right) \\
\frac{\partial\left(\boldsymbol{\theta}_{\varepsilon, k}\right)}{\partial\left(\boldsymbol{b}_{\varepsilon, k-1}\right)}=-d_{t} \boldsymbol{I}_{3 \times 3}
\end{gathered}
$$

\lfloor\rfloor$_{\times}$denotes a skew-symmetric matrix.

$$
\lfloor\boldsymbol{a}\rfloor_{\times}=\left[\begin{array}{ccc}
0 & -a_{z} & a_{y} \\
a_{z} & 0 & -a_{x} \\
-a_{y} & a_{x} & 0
\end{array}\right]
$$

Based on Eq (4.35), the measurement model can be rewritten as

$$
\begin{aligned}
\overline{\boldsymbol{y}}_{k}= & \left(\hat{\boldsymbol{q}}_{g}^{s}\right)_{k} \otimes\left[\begin{array}{ll}
1 & \frac{1}{2} \boldsymbol{\theta}_{\varepsilon, k}
\end{array}\right]+\boldsymbol{n}_{\boldsymbol{y}_{k}} \\
= & \frac{1}{2}\left[\begin{array}{l}
2 \hat{q}_{a, k}-\theta_{\varepsilon_{x}, k} \hat{q}_{b, k}-\theta_{\varepsilon_{y}, k} \hat{q}_{c, k}-\theta_{\varepsilon_{z}, k} \hat{q}_{d, k} \\
2 \hat{q}_{b, k}+\theta_{\varepsilon_{x}, k} \hat{q}_{a, k}-\theta_{\varepsilon_{y}, k} \hat{q}_{d, k}+\theta_{\varepsilon_{z}, k} \hat{q}_{c, k} \\
2 \hat{q}_{c, k}+\theta_{\varepsilon_{x}, k} \hat{q}_{d, k}+\theta_{\varepsilon_{y}, k} \hat{q}_{a, k}-\theta_{\varepsilon_{z}, k} \hat{q}_{b, k} \\
2 \hat{q}_{d, k}-\theta_{\varepsilon_{x}, k} \hat{q}_{c, k}+\theta_{\varepsilon_{y}, k} \hat{q}_{b, k}+\theta_{\varepsilon_{z}, k} \hat{q}_{a, k}
\end{array}\right]+\boldsymbol{n}_{\boldsymbol{y}_{k}}
\end{aligned}
$$

where $\hat{q}_{a, k}, \hat{q}_{b, k}, \hat{q}_{c, k}, \hat{d}_{d, k}, \theta_{\varepsilon_{x}, k}, \theta_{\varepsilon_{y}, k}, \theta_{\varepsilon_{z}, k}$ are components of $\left(\hat{\boldsymbol{q}}_{g}^{s}\right)_{k}$ and $\boldsymbol{\theta}_{\varepsilon, k}$.

$$
\left\{\begin{array}{c}
\left(\hat{\boldsymbol{q}}_{g}^{s}\right)_{k}=\left[\begin{array}{llll}
\hat{q}_{a, k} & \hat{q}_{b, k} & \hat{q}_{c, k} & \hat{q}_{d, k}
\end{array}\right]^{T} \\
\boldsymbol{\theta}_{\varepsilon, k}=\left[\begin{array}{lll}
\boldsymbol{\theta}_{\boldsymbol{\varepsilon}_{x}, k} & \theta_{\boldsymbol{\varepsilon}_{y}, k} & \theta_{\varepsilon_{z}, k}
\end{array}\right]^{T}
\end{array}\right.
$$

The components of Jacobian matrix $\boldsymbol{H}_{\varepsilon, k}$ in Eq (4.37) are

$$
\begin{gathered}
\frac{\partial \overline{\boldsymbol{y}}_{k}}{\partial \boldsymbol{\theta}_{\varepsilon, k}}=\frac{1}{2}\left[\begin{array}{ccc}
-\hat{q}_{b, k} & -\hat{q}_{c, k} & -\hat{q}_{d, k} \\
\hat{q}_{a, k} & -\hat{q}_{d, k} & \hat{q}_{c, k} \\
\hat{q}_{d, k} & \hat{q}_{a, k} & -\hat{q}_{b, k} \\
-\hat{q}_{c, k} & \hat{q}_{b, k} & \hat{q}_{a, k}
\end{array}\right] \\
\frac{\partial \overline{\boldsymbol{y}}_{k}}{\partial \boldsymbol{\theta}_{\varepsilon, k}}=\boldsymbol{0}_{9 \times 3}
\end{gathered}
$$




\section{A.4 Determination of the orientation based on two vectors}

Using OTS, we obtain two vectors $\hat{\boldsymbol{r}}_{12}^{g}$ and $\hat{\boldsymbol{r}}_{13}^{g}$ from three markers. We can determine a marker frame based on these vectors and subsequently calculate the orientation of this frame respect to the global frame in the following manner:

Step 1: determine a normalized vector:

$$
\hat{\boldsymbol{r}}_{3}^{g}=\frac{\hat{\boldsymbol{r}}_{12}^{g}}{\left|\hat{\boldsymbol{r}}_{12}^{g}\right|}
$$

Step 2: determine a second normalized vector based on $\hat{\boldsymbol{r}}_{3}^{g}$ and $\hat{\boldsymbol{r}}_{13}^{g}$ :

$$
\hat{\boldsymbol{r}}_{1}^{g}=\frac{\hat{\boldsymbol{r}}_{13}^{g} \times \hat{\boldsymbol{r}}_{3}^{g}}{\left|\hat{\boldsymbol{r}}_{13}^{g} \times \hat{\boldsymbol{r}}_{3}^{g}\right|}
$$

Step 3: determine a third normalized vector to formulate an orthogonal coordinate frame:

$$
\hat{\boldsymbol{r}}_{2}^{g}=\frac{\hat{\boldsymbol{r}}_{3}^{g} \times \hat{\boldsymbol{r}}_{1}^{g}}{\left|\hat{\boldsymbol{r}}_{3}^{g} \times \hat{\boldsymbol{r}}_{1}^{g}\right|}
$$

The orientation from marker to global frame can be expressed in a rotation matrix based on the above three steps.

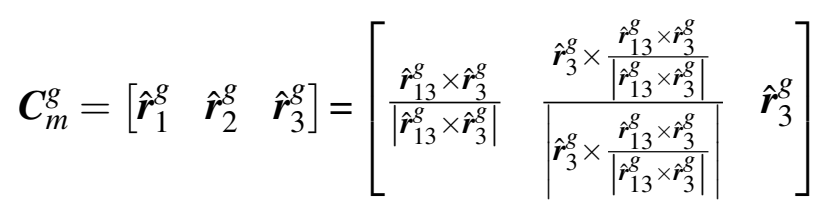

The rotation matrix $\boldsymbol{C}_{m}^{g}$ can be easily transferred into quaternion $\boldsymbol{q}_{m}^{g}$ as shown in $\mathrm{Eq}$ (4.4). 
CHAPTER 5

\section{Estimation of relative hand-finger orientation using a small IMU configuration}

This chapter is published as a journal article: Yang, Z., Van Beijnum, B. J. F., Li, B., Yan, S., \& Veltink, P. H. (2020). Estimation of Relative HandFinger Orientation Using a Small IMU Configuration. Sensors, 20(14), 4008 . 


\title{
Estimation of relative hand-finger orientation using a small IMU configuration
}

\begin{abstract}
Relative orientation estimation between the hand and its fingers is important in many applications, such as virtual reality (VR), augmented reality (AR) and rehabilitation. It is still quite a big challenge to do the estimation by only exploiting inertial measurement units (IMUs) because of the integration drift that occurs in most approaches. When the hand is functionally used, there are many instances in which hand and finger tips move together, experiencing almost the same angular velocities, and in some of these cases, almost the same accelerations are measured in different 3D coordinate systems. Therefore, we hypothesize that relative orientations between the hand and the finger tips can be adequately estimated using 3D IMUs during such designated events (DEs) and in between these events. We fused this extra information from the DEs and IMU data with an extended Kalman filter (EKF). Our results show that errors in relative orientation can be smaller than five degrees if DEs are constantly present and the linear and angular movements of the whole hand are adequately rich. When the DEs are partially available in a functional water-drinking task, the orientation error is smaller than 10 degrees.
\end{abstract}




\subsection{Introduction}

Hand-finger movement tracing is useful in many areas, such as virtual reality (VR), augmented reality (AR), ergonomic assessment and especially medical applications [129, 130, 131, 132, 133]. People who suffered from stroke or injury of the spinal cord need an effective rehabilitation therapy for recovery of body functions, including hand function. In a hospital, therapists evaluate the hand function through some traditional assessments such as the Fugl-Meyer or Jebsen-Taylor hand function assessment [134, 135]. Currently, the results may be subjective and dependent on the therapist. Therefore, it is essential to provide a quantitative and understandable measurement to make the therapist's diagnosis more objective. Several sensory systems can be used to trace hand motion, which can be categorized as camera-based, glove-based, magnetic actuator-based and inertial measurement unit (IMU)based. Camera-based systems can be divided into two different types. One uses high-speed cameras to trace markers attached to body segments, which is quite accurate and often used as the reference [136]. However, occlusion problems will influence its accuracy and the distance between cameras and hands needs to be below a few meters in order to accurately measure hand and finger orientations. Because of these problems, you need many cameras (6 to 12). The other camera-based system traces objects, including their orientations, by exploiting depth maps to reconstruct the object [137, 138]. Its advantage is that no finger or hand attachments are needed, making it friendly to users. However, this system also suffers from the occlusion problem and only allows hand movements to be evaluated if they occur in the vicinity of the cameras $[139,140]$. Besides, it requires a powerful processor to process the images. Glove-based sensor systems exploit varying sensors, such as resistive-bend sensors and optical-fiber sensors on the glove, transducing finger movement into corresponding signals to estimate relative orientations between hand and finger segments [141, 142]. It has the benefit of a low price. However, the glove needs to be well attached for the measurement and requires thorough calibration before utilization. The magnetic actuator-based system also has two types, active actuation and passive actuation. The first one deploys active magnetic actuators on the finger tip and receivers on the dorsal side of the hand [143]. It has high accuracy and no occlusion issue. However, it requires different frequencies for each degree of freedom $(\mathrm{DoF})$ of the actuator, which often needs equipment such as multiple power signal sources and a high-speed processor. This affects 
the complexity of the system and its physical dimensions. The second one uses magnets as passive sources, magnets are placed on the finger tips while magnetic sensors are worn on the wrist [144]. It has the benefit of having a simple structure and a low cost. However, it is difficult to distinguish the fields of different magnets, since only the sum of the fields are measured, especially when the magnets get close. The IMU-based system utilizes inertial sensors to trace the hand $[145,146,26]$. Compared with previous methods, it can provide raw data including angular velocity and acceleration. Orientation can be estimated by fusing the raw data. This operation suffers from drift, since it involves integration operations. However, this drift can be compensated using magnetometer data, which is easily accessible since it is often embedded in IMU systems. However, magnetometers are used in this solution and are therefore vulnerable to external magnetic disturbances, such as indoor iron surroundings [147, 101]. Thomas and Wolfgang et al. proposed magnetometer-free methods for the joint angle estimation $[148,105,149,150]$. However, such methods assume the rotation is restricted to two DoFs because of the anatomy constraint [105]. Thus, they cannot be applied to flexible joints, such as the metacarpophalangeal joint (MCP) of the thumb. Relative orientations between the hand and its fingers are important for the reconstruction of hand-finger movement, which is essential information for AR, VR and rehabilitation.

Our goal is to estimate relative $3 \mathrm{D}$ orientations between finger tips and the dorsal side of the hand with only IMUs, essentially getting rid of magnetic disturbance by not using magnetometers. In order to reduce the integration drift, we exploit information during the daily life rather than using the biomechanical constraint. The information is based on the assumption that there are many instances in which hand and finger tips move together, experiencing almost the same angular velocities and accelerations represented in different 3D coordinate systems. The method was verified with a small sensor configuration: one sensor on the dorsal side of hand, and one on the most distal finger segment of interest.

\subsection{Methods}

In order to estimate the relative orientation, the information from the gyroscope and accelerometer and extra information during DEs need to be combined in an optimal way. Therefore, an extended Kalman filter (EKF) was 
introduced to estimate 3D relative orientations between the dorsal side of the hand and finger tips, assuming angular velocities and accelerations are the same, but just represented in a different coordinate system. The process model is based on integrating relative angular velocity, the measurement model is mainly based on the information during the DE. The quality of the DE is considered in the measurement variance. When the DE is available with small variance, we trust the measurement model more; otherwise, we trust the process model more. Thus, the information from process and measurement models is optimally fused to estimate relative $3 \mathrm{D}$ orientations during functional hand and finger movements.

\subsubsection{Sensor Model}

The gain error and non-orthogonality error are assumed to be time-invariant and can be obtained through sensor calibration; thus, the outputs of calibrated gyroscope can be expressed as

$$
\left\{\begin{array}{l}
\boldsymbol{y}_{g y r, h}^{h}=\boldsymbol{\omega}_{h}^{h}+\boldsymbol{b}_{h}+\zeta_{h} \\
\boldsymbol{y}_{g y r, f}^{f}=\boldsymbol{\omega}_{f}^{f}+\boldsymbol{b}_{f}+\zeta_{f}
\end{array}\right.
$$

where $\boldsymbol{y}_{g y r, h}^{h}$ and $\boldsymbol{y}_{g y r, f}^{f}$ are gyroscope outputs on the hand and finger tip in their own frames. $\boldsymbol{b}_{x}(x=h, f)$ is the slowly varying offset. $\zeta_{x}(x=h, f)$ is Gaussian noise.

For the calibrated accelerometer, the outputs on the hand and finger tips are

$$
\left\{\begin{array}{l}
\boldsymbol{y}_{a c c, h}^{h}=\boldsymbol{a}_{h}^{h}+\boldsymbol{g}^{h}+\boldsymbol{\eta}_{h}^{h} \\
\boldsymbol{y}_{a c c, f}^{f}=\boldsymbol{a}_{f}^{f}+\boldsymbol{g}^{f}+\boldsymbol{\eta}_{f}^{f}
\end{array}\right.
$$

where $\boldsymbol{g}$ is the gravity, and $\boldsymbol{\eta}_{h}^{h}$ and $\boldsymbol{\eta}_{f}^{f}$ are Gaussian noise.

\subsubsection{Process Model}

The process model is based on integrating the relative angular velocity between the hand and its fingers in its own frame. We choose the quaternion $\boldsymbol{q}_{h f}=\left[\begin{array}{llll}q_{0} & q_{1} & q_{2} & q_{3}\end{array}\right]^{T}$ that expresses relative orientation from a finger tip to the dorsal side of the hand as the state vector $\boldsymbol{x}=\boldsymbol{q}_{h f}$. The relative orientation $\boldsymbol{x}_{k}$ is updated as

$$
\boldsymbol{x}_{k}=\boldsymbol{x}_{k-1} \otimes\left[\begin{array}{cc}
1 & \frac{1}{2} \boldsymbol{\omega}_{k} d_{t}
\end{array}\right]+\boldsymbol{m}
$$


where $\boldsymbol{m}$ is the process error. $\boldsymbol{\omega}_{k}$ is the relative angular velocity between the hand and fingers; $\otimes$ represents the multiplication operator between two quaternions.

$$
\boldsymbol{\omega}_{k}=\left(\boldsymbol{\omega}_{h}^{h}\right)_{k}-\boldsymbol{x}_{k-1} \otimes\left(\boldsymbol{\omega}_{f}^{f}\right)_{k} \otimes \boldsymbol{x}_{k-1}^{*}
$$

where $\boldsymbol{\omega}_{h}^{h}$ and $\boldsymbol{\omega}_{f}^{f}$ are hand and finger angular velocities.

\subsubsection{Measurement Model}

The measurement update of EKF is based on the DE. During the DE, the hand and fingers share the same angular velocity in different coordinate frames

$$
\boldsymbol{\omega}_{h}^{h}=\boldsymbol{q}_{h f} \otimes \boldsymbol{\omega}_{f}^{f} \otimes \boldsymbol{q}_{h f}^{*}
$$

where $\boldsymbol{\omega}_{x}^{y}(x=h, f, y=h, f)$ is the angular velocity of an object in frame $x$ expressed in the coordinate frame of object $y$. $h$ represents the hand and $f$ represents the finger tip. Combining Equations (5.1) and (5.5), we find:

$$
\begin{aligned}
\boldsymbol{y}_{g y r, h}^{h} & =\boldsymbol{q}_{h f} \otimes \boldsymbol{y}_{g y r, f}^{f} \otimes \boldsymbol{q}_{h f}^{*}+\boldsymbol{b}_{h}-\boldsymbol{q}_{h f} \otimes \boldsymbol{b}_{f} \otimes \boldsymbol{q}_{h f}^{*}+ \\
& \zeta_{h}-\boldsymbol{q}_{h f} \otimes \zeta_{f} \otimes \boldsymbol{q}_{h f}^{*} \\
& =\boldsymbol{q}_{h f} \otimes \boldsymbol{y}_{g y r, f}^{f} \otimes \boldsymbol{q}_{h f}^{*}+\boldsymbol{d}_{g y r}
\end{aligned}
$$

where the combined error of gyroscope $\boldsymbol{d}_{\text {gyr }}$ is

$$
\boldsymbol{d}_{g y r}=\left(\boldsymbol{b}_{h}-\boldsymbol{q}_{h f} \otimes \boldsymbol{d}_{f} \otimes \boldsymbol{q}_{h f}^{*}\right)+\left(\zeta_{h}-\boldsymbol{q}_{h f} \otimes \zeta_{f} \otimes \boldsymbol{q}_{h f}^{*}\right)
$$

Unlike the angular velocity, accelerations at different positions are different, which can be expressed as

$$
\begin{aligned}
& \boldsymbol{a}_{h}^{h}=\boldsymbol{q}_{h f} \otimes \boldsymbol{a}_{f}^{f} \otimes \boldsymbol{q}_{h f}^{*}+\boldsymbol{\omega}_{h}^{h} \times\left(\boldsymbol{\omega}_{h}^{h} \times \boldsymbol{r}_{f h}^{h}\right)+\dot{\boldsymbol{\omega}}_{h}^{h} \times \boldsymbol{r}_{f h}^{h} \\
& =\boldsymbol{q}_{h f} \otimes \boldsymbol{a}_{f}^{f} \otimes \boldsymbol{q}_{h f}^{*}+\left(\left\lfloor\boldsymbol{\omega}_{h}^{h}\right\rfloor_{\times}\left\lfloor\boldsymbol{\omega}_{h}^{h}\right\rfloor_{\times}+\left[\dot{\boldsymbol{\omega}}_{h}^{h}\right\rfloor_{\times}\right) \boldsymbol{r}_{f h}^{h}
\end{aligned}
$$

where $\boldsymbol{a}_{x}^{y}(x=h, f, y=h, f)$ is the acceleration of object in frame $x$ relative to frame $y . \dot{\boldsymbol{\omega}}_{h}^{h}$ is the hand angular acceleration in its own frame. $\boldsymbol{r}_{f h}^{h}$ is the position vector between hand and fingers in the hand frame. $\rfloor_{\times}$denotes a skew-symmetric matrix.

$$
\lfloor\boldsymbol{a}\rfloor_{\times}=\left[\begin{array}{ccc}
0 & -a_{z} & a_{y} \\
a_{z} & 0 & -a_{x} \\
-a_{y} & a_{x} & 0
\end{array}\right]
$$


If the second term $\left(\left\lfloor\boldsymbol{\omega}_{h}^{h}\right\rfloor_{\times}\left\lfloor\boldsymbol{\omega}_{h}^{h}\right\rfloor_{\times}+\left\lfloor\dot{\boldsymbol{\omega}}_{h}^{h}\right\rfloor_{\times}\right) \boldsymbol{r}_{f h}^{h}$ is relatively small compared with the first term $\boldsymbol{q}_{h f} \otimes \boldsymbol{a}_{f}^{f} \otimes \boldsymbol{q}_{h f}^{*}$, then Equation (5.8) can be approximated as the following equation:

$$
\boldsymbol{a}_{h}^{h} \approx \boldsymbol{q}_{h f} \otimes \boldsymbol{a}_{f}^{f} \otimes \boldsymbol{q}_{h f}^{*}
$$

Combining Equations (5.2) and (5.8), we find:

$$
\boldsymbol{y}_{a c c, h}^{h}=\boldsymbol{q}_{h f} \otimes \boldsymbol{y}_{a c c, f}^{f} \otimes \boldsymbol{q}_{h f}^{*}+\left(\left\lfloor\boldsymbol{\omega}_{h}^{h}\right\rfloor_{\times}\left\lfloor\boldsymbol{\omega}_{h}^{h}\right\rfloor_{\times}+\left\lfloor\dot{\boldsymbol{\omega}}_{h}^{h}\right\rfloor_{\times}\right) \boldsymbol{r}_{f h}^{h}+\boldsymbol{\eta}_{c}
$$

where the combined error $\boldsymbol{\eta}_{c}$ can be expressed as

$$
\boldsymbol{\eta}_{c}=\boldsymbol{\eta}_{h}^{h}-\boldsymbol{q}_{h f} \otimes \boldsymbol{\eta}_{f}^{f} \otimes \boldsymbol{q}_{h f}^{*}
$$

Finally, an overall relation between hand and fingers based on Equations (5.6) and (5.11) is

$$
\left\{\begin{array}{c}
\boldsymbol{y}_{g y r, h}^{h}=\boldsymbol{q}_{h f} \otimes \boldsymbol{y}_{g y r, f}^{f} \otimes \boldsymbol{q}_{h f}^{*}+\boldsymbol{d}_{g y r} \\
\boldsymbol{y}_{a c c, h}^{h}=\boldsymbol{q}_{h f} \otimes \boldsymbol{y}_{a c c, f}^{f} \otimes \boldsymbol{q}_{h f}^{*}+\left(\left\lfloor\boldsymbol{\omega}_{h}^{h}\right\rfloor_{\times}\left\lfloor\boldsymbol{\omega}_{h\rfloor_{\times}^{h}}^{h}+\left\lfloor\dot{\boldsymbol{\omega}}_{h}^{h}\right\rfloor_{\times}\right) \boldsymbol{r}_{f h}^{h}+\boldsymbol{\eta}_{c}\right.
\end{array}\right.
$$

Subsequently, we can get the measurement model based on the sensor model and quaternion constraint

$$
\boldsymbol{y}_{k}=f\left(\boldsymbol{x}_{k}\right)+\boldsymbol{v}
$$

where $\boldsymbol{y}$ and $f$ can be expressed as

$$
\begin{aligned}
& \boldsymbol{y}_{k}=\left[\begin{array}{lll}
\left(y_{a c c, h}^{h}\right)^{T} & \left(\boldsymbol{y}_{g y r, h}^{h}\right)^{T} & 0
\end{array}\right]^{T} \\
& f(\boldsymbol{x})=\left[\begin{array}{c}
\boldsymbol{x}_{k} \otimes \boldsymbol{y}_{a c c, f}^{f} \otimes \boldsymbol{x}_{k}^{*} \\
\boldsymbol{x}_{k} \otimes \boldsymbol{y}_{g y r_{f} f}^{f} \otimes \boldsymbol{x}_{k}^{*} \\
q_{0}^{2}+q_{1}^{2}+q_{2}^{2}+q_{3}^{2}-1
\end{array}\right]
\end{aligned}
$$

As shown in Equations (5.3) and (5.16), the process and measurement model are both nonlinear with respect to $\boldsymbol{x}_{k}$. In order to update the covariance matrix for $\boldsymbol{x}_{k}$, linearization is performed and the Jacobian matrix $\boldsymbol{F}$ and $\boldsymbol{H}$ for process and measurement model are calculated; the details can be found in the Appendix B. 


\subsubsection{Uncertainty Error Variance}

In order to assess the relative confidence in the measurement model (based on our DE assumptions) and the process model, the measurement variance is determined. According to the assumption that a hand and finger share approximately the same angular velocity and acceleration based on Equation (5.13), the differences in angular velocity and acceleration between the hand and fingers measured by the IMU determine the measurement variance. From Equation (5.7), the error is related to the offset error, the white noise and relative orientation. $\boldsymbol{d}_{g y r}$ can be expressed with following equation from Equation (5.6).

$$
\boldsymbol{d}_{g y r}=\boldsymbol{y}_{g y r, h}^{h}-\boldsymbol{q}_{h f} \otimes \boldsymbol{y}_{g y r, f}^{f} \otimes \boldsymbol{q}_{h f}^{*}
$$

We approximate the distribution of $\boldsymbol{d}_{g y r}$ as Gaussian distribution with zero mean and standard deviation $\sigma_{g}\left[\begin{array}{lll}1 & 1 & 1\end{array}\right](\mathrm{rad} / \mathrm{s})$

$$
\sigma_{g}=\left\|\boldsymbol{y}_{g y r, h}^{h}-\boldsymbol{q}_{h f} \otimes \boldsymbol{y}_{g y r, f}^{f} \otimes \boldsymbol{q}_{h f}^{*}\right\|_{2}
$$

For Eq (5.13), the error $\boldsymbol{d}_{a c c}$ can be expressed with the following equation:

$$
\boldsymbol{d}_{a c c}=\left(\left\lfloor\boldsymbol{\omega}_{h}^{h}\right\rfloor_{\times}\left\lfloor\boldsymbol{\omega}_{h}^{h}\right\rfloor_{\times}+\left\lfloor\dot{\boldsymbol{\omega}}_{h}^{h}\right\rfloor_{\times}\right) \boldsymbol{r}_{f h}^{h}+\boldsymbol{\eta}
$$

We can express the error in another format from Equation (5.11).

$$
\boldsymbol{d}_{a c c}=\boldsymbol{y}_{a c c, h}^{h}-\boldsymbol{q}_{h f} \otimes \boldsymbol{y}_{a c c, f}^{f} \otimes \boldsymbol{q}_{h f}^{*}
$$

Similarly to the gyroscope, we assume the error $\boldsymbol{d}_{a c c}$ has an approximate Gaussian distribution with zero mean while its standard deviation $\sigma_{a}\left[\begin{array}{lll}1 & 1 & 1\end{array}\right]$ is

$$
\sigma_{a}=\left\|\boldsymbol{y}_{a c c, h}^{h}-\boldsymbol{q}_{h f} \otimes \boldsymbol{y}_{a c c, f}^{f} \otimes \boldsymbol{q}_{h f}^{*}\right\|_{2}
$$

Based on the Gaussian approximation, as described in Equations (5.17) and (5.20), it is essential to know the rotation quaternion $\boldsymbol{q}_{h f}$ before we get the variance. However, $\boldsymbol{q}_{h f}$ is the variable we try to estimate which is also unknown. As we assume there is no or a slow orientation change between the hand and finger tips, the estimated relative orientation at time $k-1$ is used as the true relative orientation at time $k$.

$$
\boldsymbol{q}_{h f, k}=\hat{\boldsymbol{q}}_{h f, k-1}
$$


where $\boldsymbol{q}_{h f, k}$ is the "true" rotation quaternion we use to estimate the variance at time $k . \hat{\boldsymbol{q}}_{h f, k-1}$ is the estimated rotation quaternion at time $k-1$. The measurement covariance is determined as

$$
\boldsymbol{R}_{m}=\left[\begin{array}{ccc}
\sigma_{g} \boldsymbol{I}_{3 \times 3} & \boldsymbol{0} & \boldsymbol{0} \\
\boldsymbol{0} & \boldsymbol{\sigma}_{a} \boldsymbol{I}_{3 \times 3} & \boldsymbol{0} \\
\boldsymbol{0} & \boldsymbol{0} & 0
\end{array}\right]
$$

The initial value for the state vector of relative orientations $\boldsymbol{x}_{k}$ was set as $\left[\begin{array}{llll}1 & 0 & 0 & 0\end{array}\right]^{T}$.

\subsection{Experiments}

\subsubsection{Experiment Setup}

The sensor system includes three IMUs fixed on the most distal segments of the thumb and index finger and the dorsal side of the hand, as shown in Figure 5.1. MPU9250 (InvenSense) was chosen for the IMU, which contains a tri-axis accelerometer and tri-axis gyroscope (it also contains a tri-axis magnetometer, which was not used in the current study). All IMUs were sampled synchronously; the sample frequencies of gyroscope and accelerometer were $200 \mathrm{~Hz}$ and $100 \mathrm{~Hz}$ respectively. All the data were collected by a master micro-controller (Atmel XMEGA) and then transmitted to the PC via a USB connection. Prior to the experiment, the accelerometer was calibrated based on local gravity; the gyroscope was calibrated based on the calibrated accelerometer [151]. An optical Vicon system with eight cameras was used to perform 3D orientation reference measurements. For this purpose, three optical markers were attached to each IMU. The sampling frequency of Vicon was $100 \mathrm{~Hz}$. 


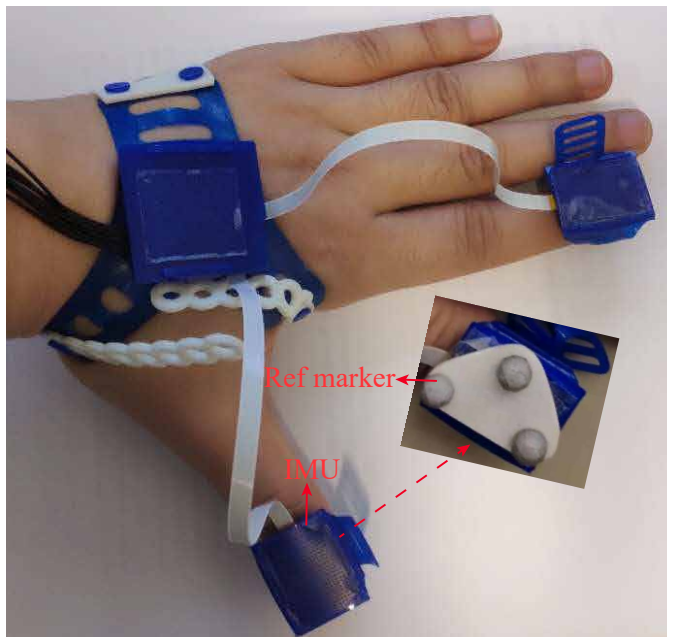

Figure 5.1: IMUs on the dorsal side of the hand and fingertips. The inset shows the cluster of optical markers used on top of each IMU for reference measurement of segment orientations using the optical VICON system. Every cluster contains three markers, which determine a $3 D$ coordinate frame.

\subsubsection{Alignment of the IMU and Reference Marker Frame for the Validation Experiment}

For evaluation of the IMU-based 3D relative orientation estimation using the optical system, it is essential to calibrate the relative orientation between the sensor and marker-based reference frame. Here, we used the accelerometer for this marker system's IMU calibration. Holding the system static, we obtained the gravity in the IMU frame from the accelerometer readings. Meanwhile, we obtained the orientation from the global Vicon frame to marker frame $\boldsymbol{q}_{m g}$. Gravity in marker frame is $\boldsymbol{q}_{m g} \otimes \boldsymbol{g} \otimes \boldsymbol{q}_{m g}^{*}$, where $\boldsymbol{g}$ is gravity in global Vicon frame ( $z$-axis of global Vicon system was vertical upward; gravity in this frame was $g=\left[\begin{array}{ccc}0 & 0 & -g\end{array}\right] ; g$ is the local gravity value). When we have at least two poses, we obtain more than two vectors expressed in the marker frame and IMU frame respectively, which is enough to determine the relative orientation between the IMU and marker frame. 


\subsubsection{Sensor to Segment Calibration}

Before the experiment, IMU errors were calibrated according to D Tedaldi et al.'s and WT Fong et al.'s research [151, 152], including sensitivity error, offset error, non-orthogonal error and misalignment between the accelerometer and gyroscope. After the IMU was fixed on the hand and fingers, the relative orientations between IMU sensors and body segments were calibrated. An accelerometer was used to achieve the alignment by exploiting static accelerometer measures of gravity. When we held our hand sequentially horizontally and vertically, we obtained the 3D relative orientation between two frames. More details can be found in Kortier et al.'s research [102].

\subsubsection{Synchronization of Vicon and IMU System}

In this experiment, the two measurement systems were synchronized by recording the sensed responses of an induced impact at the start and end of each experiment. At the start and end of every experiment, we hit the IMU on a desk, resulting in an acceleration peak measured by the IMU system and a minimum vertical position of the Vicon markers simultaneously, which was used to synchronize the two systems.

\subsubsection{Protocols for the Experiment}

In order to demonstrate the feasibility of our approach, an experimental part was designed to estimate the accuracy of the algorithm compared with the optical system. Our feasibility experiment involved three participants. The protocol was reviewed, approved and conducted under the auspices of the Ethics Committee EEMCS, Univerisity of Twente. The following tasks were performed:

Task1: Movements and rotations of the hand, while not varying relative orientations between hand and fingers: IMUs were fixed on fingers and the dorsal side of the hand. Then, the participant did the pronation and supination movements with the arm while the axis of pronation and supination was continuously changing. The orientation was changed over approximately $160^{\circ}$ around the rotation axis; see Figure 5.2. Furthermore, we varied the angular velocity by performing these cyclical movements with varying repetition rate of pronation and supination $(60,120,240$ cycles/min), with the help of a metronome. This was done in order to test the performance of the 
algorithm under different conditions. During the process, the subject was asked to close the hand and not change the relative orientations between the hand and fingers, while displacing or rotating the hand.

Task2: Simple functional task. The subject was asked to place the hand on the desk; then rise the hand and grasp a cup; subsequently drink some water and place the cup back; and finally place the hand on the original position. The illustration of the movement can be seen in Figure 5.3.

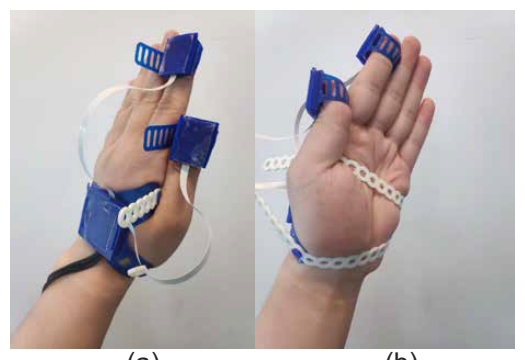

(a) (b)

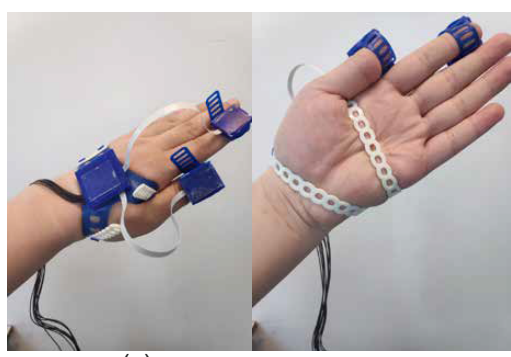

(d)

Figure 5.2: Movement for task 1: rotations of the hand, while not varying relative orientations between the hand and fingers. Subfigure $(\boldsymbol{a})$ and $(\boldsymbol{b})$ are a set of pronation and supination movements. Subfigure $(\boldsymbol{c})$ and $(\boldsymbol{d})$ are another set of pronation and supination but with a different rotation axis. During this task, we did the pronation and supination movements with different rotation axes.

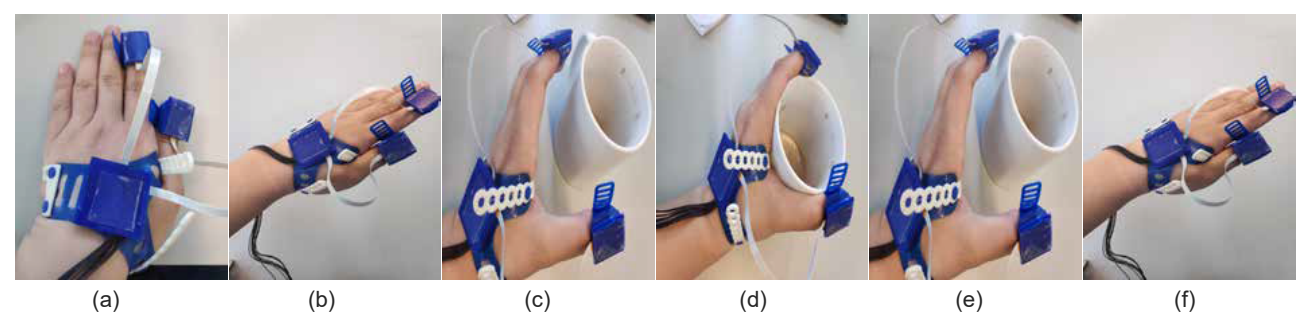

Figure 5.3: Movement for task 2: Simple functional task. The task can be divided into several phases. (a) Put the hand static on the desk; (b) raise the hand; (c) grasp the cup; (d) drink the water; (e) release the hand; $(\boldsymbol{f})$ withdraw the hand.

For task 1, the orientation reference was directly derived from the IMUs, because the relative orientation was imposed by the hand, and therefore, 
known and not varying. For task 2, the reference measurement was performed using the optical VICON system (software version 2.8.2).

\subsection{Results}

\subsubsection{Movements and Rotations of the Hand, While Not Vary- ing Relative Orientations between the Hand and Fin- ger (Task 1)}

The error angle used was the arccos of the first component of quaternion error $\boldsymbol{q}_{\text {err }}$ [153]:

$$
\boldsymbol{q}_{\text {err }}=\boldsymbol{q}_{\text {est }}^{-1} \otimes \boldsymbol{q}_{\text {ref }}=\left[\begin{array}{ll}
1 & \frac{1}{2} \boldsymbol{\theta}_{\text {err }}
\end{array}\right]
$$

where $\boldsymbol{q}_{\text {est }}$ was the estimated relative orientation and $\boldsymbol{q}_{\text {ref }}$ was the orientation reference.

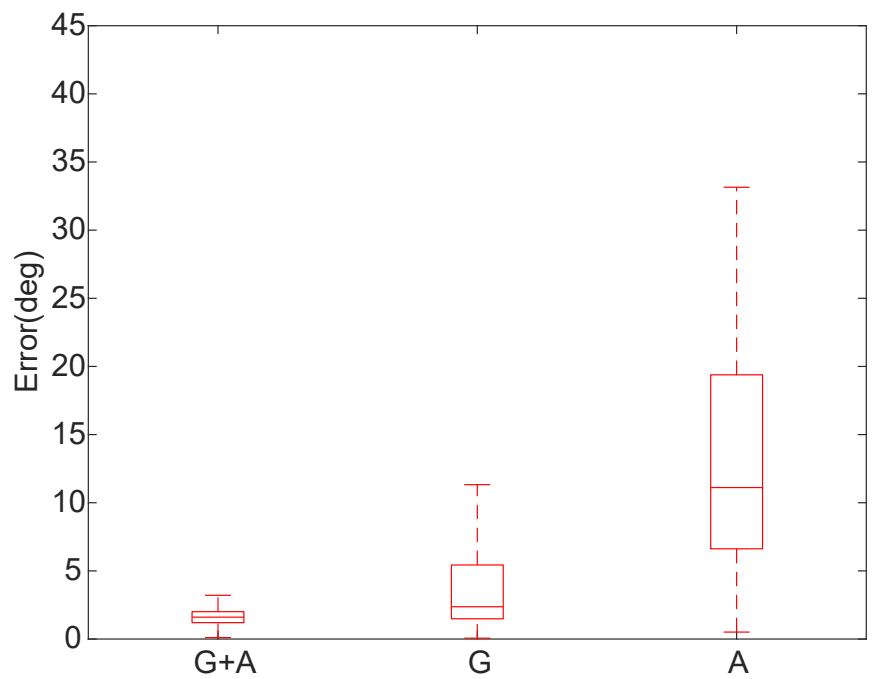

Figure 5.4: Estimated orientation error $\left|\boldsymbol{\theta}_{\text {err }}\right|$ with gyroscope and accelerometer (values under 99.3 percent coverage are shown in the boxplot figures). "G," "A" and " $G+A$ " represent estimated results based on gyroscope, accelerometer and gyroscope plus accelerometer respectively.

We obtained more than two independent vectors from the gyroscope, accelerometer or both from 3D movements. The error angle estimated when 
DE is available is shown in Figure 5.4. The orientation error is smallest with the gyroscope and accelerometer, while the orientation error is largest with accelerometer data only.

\section{Influence of Repetition Rate of Movement}

The estimation may be influenced by the repetition rate of movements. Figures 5.5 and 5.6 show the relation between the norm of gyroscope or accelerometer on thte hand and finger for several repetition rates. Ideally, the gyroscope output norms $\left\|\boldsymbol{y}_{g y r, h}\right\|,\left\|\boldsymbol{y}_{g y r, f}\right\|$ should be equal for the measurement update and for the accelerometer. The differential output norms cause estimation errors, as shown in Equation (5.13). For the accelerometer, the different output norms $\left|\left\|\boldsymbol{y}_{a c c, h}\right\|-\left\|\boldsymbol{y}_{a c c, f}\right\|\right|$ were $29.3 \mathrm{~m} / \mathrm{s}^{2}, 66.4 \mathrm{~m} / \mathrm{s}^{2}$ and $370.2 \mathrm{~m} / \mathrm{s}^{2}$ under the repetition rates 60,120 and 240 beats $/ \mathrm{min}$ respectively. Meanwhile, the correspondingly differential output norms of gyroscope were $2.2 \mathrm{rad} / \mathrm{s}, 2.7 \mathrm{rad} / \mathrm{s}$ and $4.4 \mathrm{rad} / \mathrm{s}$. As shown in Figure 5.7b,c, the estimated orientation error based on the accelerometer became larger when the repetition rate increased, while orientation error based on gyroscope changed little when the repetition rate increased. As shown in Figure 5.7a, the estimated result based on the gyroscope and accelerometer trusted the gyroscope more than the accelerometer because it contained less error; thus, it was also insensitive to repetition rate. 
5.4.1 Movements and Rotations of the Hand, While Not Varying Relative Orientations between the Hand and Finger (Task 1)

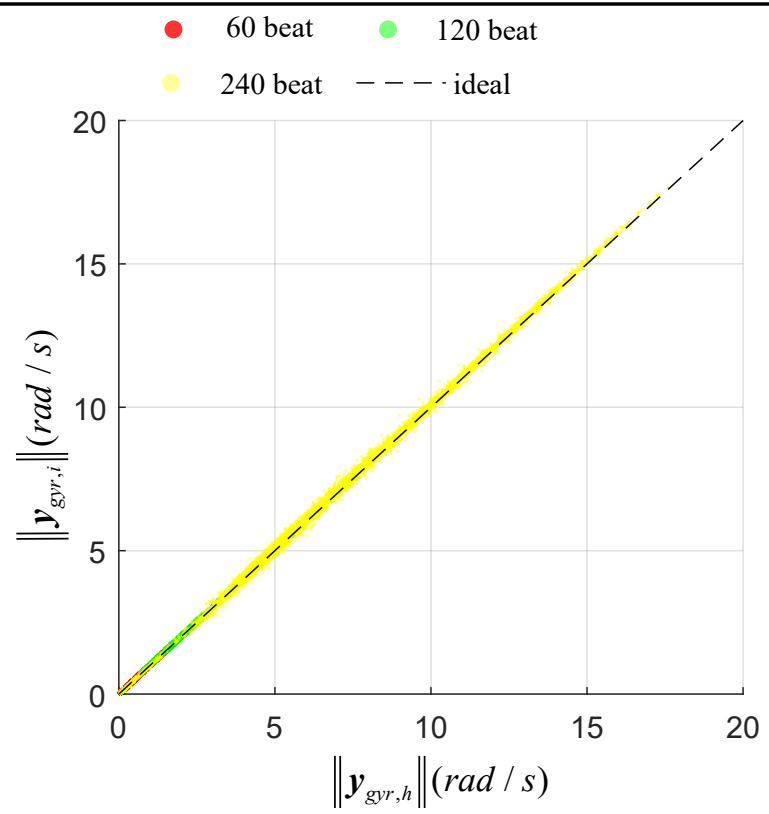

Figure 5.5: Relation of output norms between gyroscopes on the dorsal side of the hand and finger tip with different repetition rates.

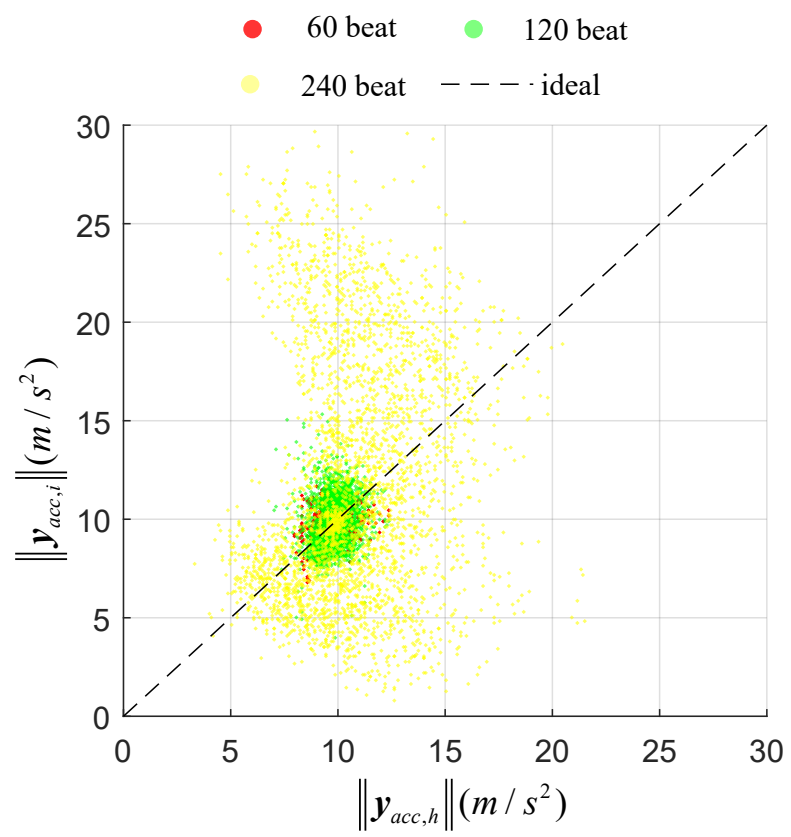

Figure 5.6: Relation of output norms between accelerometers on the dorsal side of the hand and finger tip with different repetition rates. 


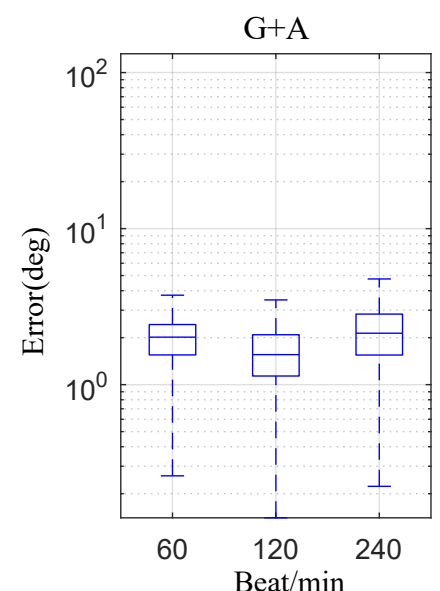

(a)

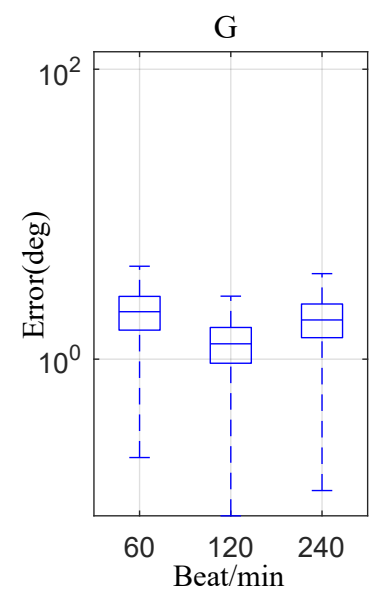

(b)

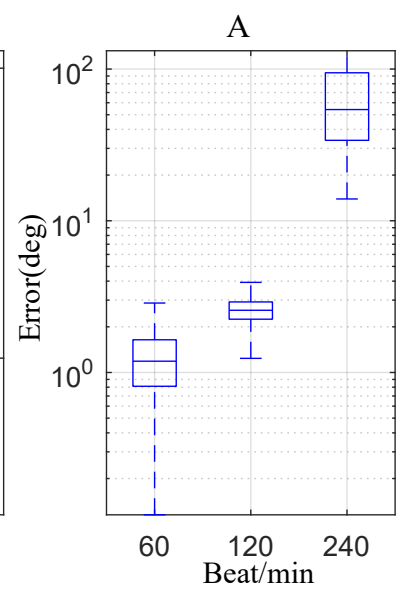

(c)

Figure 5.7: Estimation error $\left|\boldsymbol{\theta}_{\text {err }}\right|$ with different repetition rates (values under 99.3 percent coverage are shown in the boxplot figures). Subfigures $(\boldsymbol{a}),(\boldsymbol{b})$ and $(\boldsymbol{c})$ are estimations with gyroscope plus accelerometer, and gyroscope and accelerometer individually.

\subsubsection{Simple Functional Task (Task 2)}

According to Figure 5.3, the whole process was divided into several phases; the estimated orientation errors based on the optical system in different phases are shown in Figure 5.8. The error during the drinking part was relatively low because the cub imposed a constant relative orientation on the hand and fingers and the whole hand moved with varying position and orientation, as shown in Figure 5.8. Since the angular velocity and acceleration norms were close to each other, the standard deviations of measurement noise $\sigma_{a}$ and $\sigma_{g}$ were small, as shown in subfigure (b); the measurement model was trusted relatively more relative to the process model under said condition. For the other phases of this functional task, there were bigger differences between gyroscope and accelerometer norms on the hand and fingers; thus $\sigma_{a}$ and $\sigma_{g}$ were bigger; the trust in the process model was relatively high. A good estimation of relative orientation was achieved by choosing a suitable standard deviation for the process error (see Figure 5.8c). 

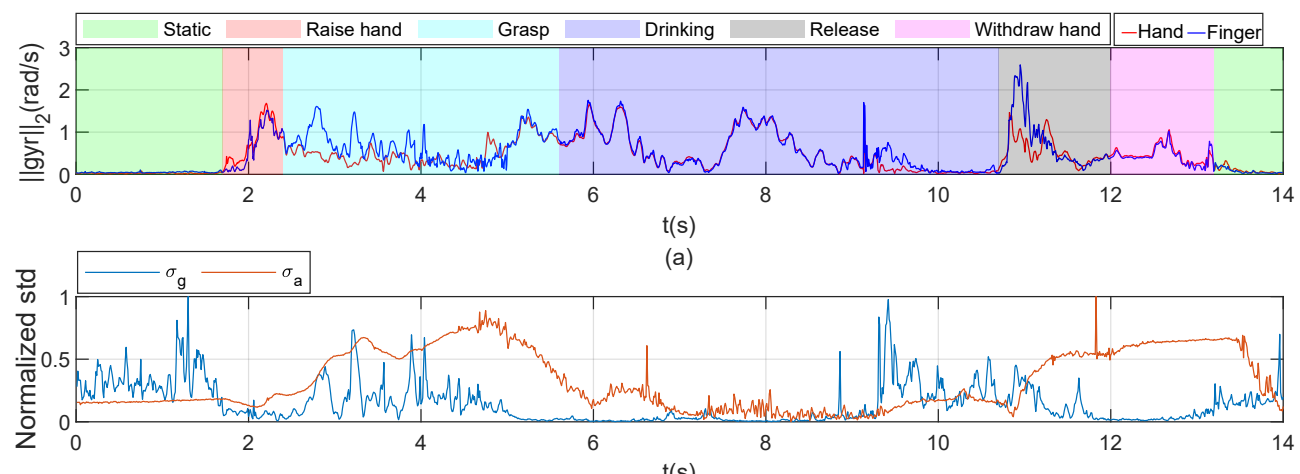

(b)

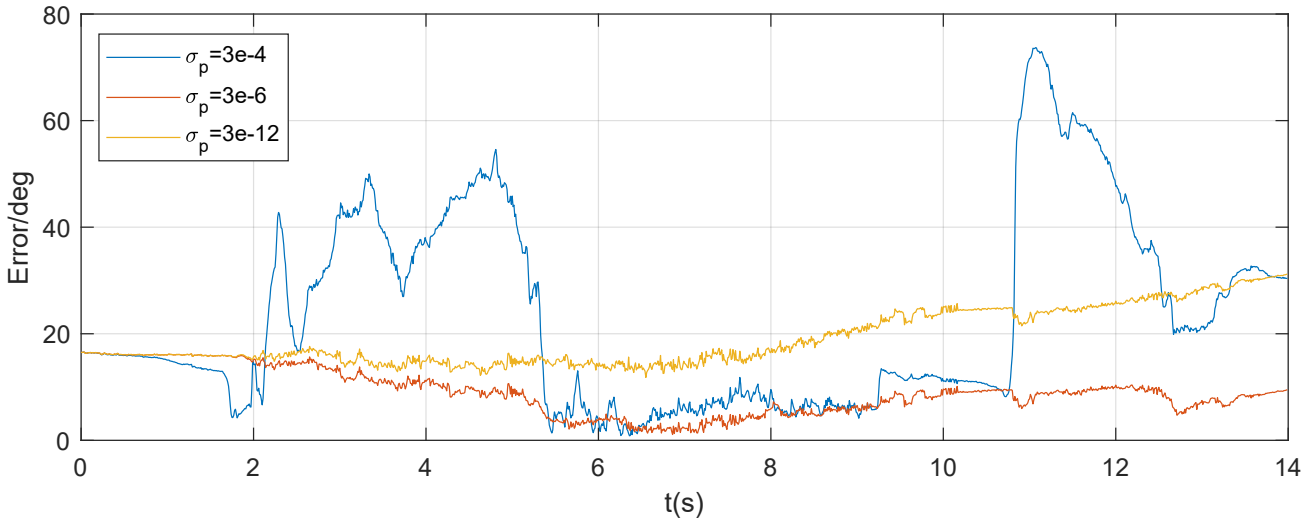

(c)

Figure 5.8: Relative orientation between hand and thumb during the waterdrinking process. Subfigure (a) shows the output norms of the two gyroscopes (on the hand and finger tip respectively). Subfigure (b) shows the normalized $S D s \sigma_{a}$ and $\sigma_{g}$ from Equations (5.18) and (5.21). Larger $\sigma_{a}$ and $\sigma_{g}$ mean larger measurement error. The EKF trusts the process model more and the measurement model less when $\sigma_{a}$ and $\sigma_{g}$ are larger. Subfigure (c) shows the estimated results with different SDs of the process model. The variance of process error $\boldsymbol{Q}$ was determined as $\sigma_{p} \boldsymbol{I}_{4}$.

\subsection{Discussion}

We proposed and evaluated an IMU-based setup for estimating 3D relative orientation between hand and finger tips. Compared with the IMU-based data glove system described by Salchov-Homer et al. [26] and Kortier et 
al. [102], we reduced the number of IMUs as much as possible and avoided magnetic disturbance, but still obtained comparable precision of estimated orientation. In reference [26], the orientation error magnitude is approximately five to ten degrees. In our research, the orientation error is related to the movement quality. When the hand and fingers move together, the median orientation error can be smaller than five degrees. For the water-drinking experiment, the estimated error is less than ten degrees when hand and fingers approximately move together, but around ten degrees during the rest periods. In our view, this is a promising method for the hand finger orientation estimation with a small IMU configuration which can be used if rich wholehand movements occur and the change of relative orientations between hand and finger tips is regular and relatively small. Standard deviations $\sigma_{g}$ and $\sigma_{a}$ can be used to assess whether such DEs regularly apply during a specify movement.

Most previous IMU-based systems [145, 102] for finger orientation estimation usually require a magnetometer to reduce the drift caused by the gyroscope, which will suffer from the magnetic disturbance problem in indoor environments. To our knowledge, in order to remove magnetic disturbance but still suppress the drift, a biomechanical model is additionally used in methods described in the literature (e.g., [145, 102]). We have not applied additional information from a bioimechanical model in our current study, although this additional information could be applied. However, it should be noted that finger movements are usually assumed to be restricted to two DoFs while using biomechanical constraints. In construct, our method can be implemented without biomechanical constraints and can be applied to estimating three-DoF-relative orientation during 3D hand movements without such biomechanical assumptions.

For the result in task 1, the relative orientation estimation is less sensitive to an increase of repetition rate of the same movement when using gyroscopes or gyroscopes plus an accelerometer than the accelerometer only. That is because as the difference among the accelerometer signals from the hand and finger becomes larger, the non-gravitational acceleration caused by increasing angular velocity or angular acceleration becomes relatively more important compared to the gravity component.

Position estimation only based on inertial sensors is quite challenging and limited by integration drift. Our further research will concentrate on relative position estimation based on IMUs combined with sensing the magnetic field of a magnetic source. For this to be feasible, an adequate estimate 
of relative orientation is required, so the 3D magnetic field measurement can be expressed in the coordinate system of the magnetic source. This is an essential first step in estimating relative positions. In this research, only one healthy participant was involved since we are mainly concentrating on verifying the performance of the algorithm. Subsequently, the proposed relative orientation and position estimation methods for the hand and finger using a small sensing configuration need to be evaluated in healthy subjects and patients during more complex daily tasks, in order to assess the applicability in clinical and daily-life settings. To make the system more friendly to users, the system could be wireless in the future.

\subsection{Conclusions}

In conclusion, IMUs can be used to estimate the relative orientation between the hand and fingers without using magnetometers. Compared with previous systems, we only exploit IMUs on finger tips and the dorsal side of the hand rather than having IMUs on every segment. The performance is dependent on how well the hand and fingers move together, which influences the accuracy of the estimate. The median value of estimation error can be smaller than five degrees when IMUs are on our hand and fingers if their relative orientation is not variant over time, while the object or hand is moving. During the water-drinking task, the estimation error can be smaller than 10 degrees during periods when the hand and fingers approximately move together, which may be adequately accurate to provide useful information to clinicians when judging.

\section{Appendix B}

\section{Parameters of EKF}

By linearizing the nonlinear function of the process model and measurement model, we obtained the Jacobian matrixes $\boldsymbol{F}$ and $\boldsymbol{H}$ respectively, which were used in EKF for the covariance update. Based on Equation (5.3): 


$$
\begin{aligned}
\boldsymbol{F} & =\frac{\partial \boldsymbol{x}_{k}}{\partial \boldsymbol{x}_{k-1}}=\frac{1}{2}\left[\begin{array}{llll}
\boldsymbol{a}_{11} \boldsymbol{\omega}_{2} & \boldsymbol{a}_{12} \boldsymbol{\omega}_{2} & \boldsymbol{a}_{13} \boldsymbol{\omega}_{2} & \boldsymbol{a}_{14} \boldsymbol{\omega}_{2} \\
\boldsymbol{a}_{21} \boldsymbol{\omega}_{2} & \boldsymbol{a}_{22} \boldsymbol{\omega}_{2} & \boldsymbol{a}_{23} \boldsymbol{\omega}_{2} & \boldsymbol{a}_{24} \boldsymbol{\omega}_{2} \\
\boldsymbol{a}_{31} \boldsymbol{\omega}_{2} & \boldsymbol{a}_{32} \boldsymbol{\omega}_{2} & \boldsymbol{a}_{33} \boldsymbol{\omega}_{2} & \boldsymbol{a}_{34} \boldsymbol{\omega}_{2} \\
\boldsymbol{a}_{41} \boldsymbol{\omega}_{2} & \boldsymbol{a}_{42} \boldsymbol{\omega}_{2} & \boldsymbol{a}_{43} \boldsymbol{\omega}_{2} & \boldsymbol{a}_{44} \boldsymbol{\omega}_{2}
\end{array}\right] d_{t} \\
& +\frac{1}{2}\left[\begin{array}{cccc}
0 & -\omega_{1 x} & -\omega_{1 y} & -\omega_{1 z} \\
\omega_{1 x} & 0 & \omega_{1 z} & -\omega_{1 y} \\
\omega_{1 y} & -\omega_{1 z} & 0 & \omega_{1 x} \\
\omega_{1 z} & \omega_{1 y} & -\omega_{1 x} & 0
\end{array}\right] d_{t}+\boldsymbol{I}_{4 \times 4}
\end{aligned}
$$

$$
\left\{\begin{array}{ccc}
\boldsymbol{a}_{11}=2\left[\begin{array}{lll}
q_{0} q_{1} & q_{0} q_{2} & q_{0} q_{3}
\end{array}\right] & \boldsymbol{a}_{12}=\left[\begin{array}{lll}
1+2 q_{1}^{2} & 2 q_{1} q_{2} & 2 q_{1} q_{3}
\end{array}\right] \\
\boldsymbol{a}_{13}=\left[\begin{array}{llll}
2 q_{1} q_{2} & 1+2 q_{2}^{2} & 2 q_{2} q_{3}
\end{array}\right] & \boldsymbol{a}_{14}=\left[\begin{array}{lll}
2 q_{1} q_{3} & 2 q_{2} q_{3} & 1+2 q_{3}^{2}
\end{array}\right] \\
\boldsymbol{a}_{21}=-\left[\begin{array}{llll}
1+2 q_{0}^{2} & 2 q_{0} q_{3} & -2 q_{0} q_{2}
\end{array}\right] & \boldsymbol{a}_{22}=-2\left[\begin{array}{llll}
q_{0} q_{1} & q_{1} q_{3} & -q_{1} q_{2}
\end{array}\right] \\
\boldsymbol{a}_{23}=\left[\begin{array}{llll}
-2 q_{0} q_{2} & -2 q_{2} q_{3} & 1+2 q_{2}^{2}
\end{array}\right] & \boldsymbol{a}_{24}=-\left[\begin{array}{lll}
2 q_{0} q_{3} & 1+2 q_{3}^{2} & -2 q_{2} q_{3}
\end{array}\right] \\
\boldsymbol{a}_{31}=-\left[\begin{array}{llll}
-2 q_{0} q_{3} & 1+2 q_{0}^{2} & 2 q_{0} q_{1}
\end{array}\right] & \boldsymbol{a}_{32}=-\left[\begin{array}{lll}
-2 q_{1} q_{3} & 2 q_{0} q_{1} & 1+2 q_{1}^{2}
\end{array}\right] \\
\boldsymbol{a}_{33}=-2\left[\begin{array}{llll}
-q_{2} q_{3} & q_{0} q_{2} & q_{1} q_{2}
\end{array}\right] & \boldsymbol{a}_{34}=\left[\begin{array}{llll}
1+2 q_{3}^{2} & -2 q_{0} q_{3} & -2 q_{1} q_{3}
\end{array}\right] \\
\boldsymbol{a}_{41}=-\left[\begin{array}{llll}
2 q_{0} q_{2} & -2 q_{0} q_{1} & 1+2 q_{0}^{2}
\end{array}\right] & \boldsymbol{a}_{42}=\left[\begin{array}{llll}
-2 q_{1} q_{2} & 1+2 q_{1}^{2} & -2 q_{0} q_{1}
\end{array}\right] \\
\boldsymbol{a}_{43}=-\left[\begin{array}{llll}
1+2 q_{2}^{2} & -2 q_{1} q_{2} & q_{0} q_{2}
\end{array}\right] & \boldsymbol{a}_{44}=-2\left[\begin{array}{llll}
q_{2} q_{3} & -q_{1} q_{3} & q_{0} q_{3}
\end{array}\right]
\end{array}\right.
$$

Based on Equation (5.16):

$$
\begin{aligned}
& \boldsymbol{H}=\frac{\partial f}{\partial \boldsymbol{x}_{k}}=\left[\begin{array}{c}
\frac{\partial\left(\boldsymbol{x}_{k} \otimes \boldsymbol{y}_{a c c, f}^{f} \otimes \boldsymbol{x}_{k}^{*}\right)}{\partial \boldsymbol{x}_{k}} \\
\frac{\partial\left(\boldsymbol{x}_{k} \otimes \boldsymbol{y}_{g y r, f}^{f} \otimes \boldsymbol{x}_{k}^{*}\right)}{\partial \boldsymbol{x}_{k}} \\
\frac{\partial\left(q_{0}^{2}+q_{1}^{2}+q_{2}^{2}+q_{3}^{2}\right)}{\partial \boldsymbol{x}_{k}}
\end{array}\right] \\
&=2\left[\begin{array}{llll}
\boldsymbol{b}_{11} \boldsymbol{y}_{a c c, f}^{f} & \boldsymbol{b}_{12} \boldsymbol{y}_{a c c, f}^{f} & \boldsymbol{b}_{13} \boldsymbol{y}_{a c c, f}^{f} & \boldsymbol{b}_{14} \boldsymbol{y}_{a c c, f}^{f} \\
\boldsymbol{b}_{21} \boldsymbol{y}_{a c c, f}^{f} & \boldsymbol{b}_{22} \boldsymbol{y}_{a c c, f}^{f} & \boldsymbol{b}_{23} \boldsymbol{y}_{a c c, f}^{f} & \boldsymbol{b}_{24} \boldsymbol{y}_{a c c, f}^{f} \\
\boldsymbol{b}_{31} \boldsymbol{y}_{a c c, f}^{f} & \boldsymbol{b}_{32} \boldsymbol{y}_{a c c, f}^{f} & \boldsymbol{b}_{33} \boldsymbol{y}_{a c c, f}^{f} & \boldsymbol{b}_{34} \boldsymbol{y}_{a c c, f}^{f} \\
\boldsymbol{b}_{11} \boldsymbol{y}_{g y r, f}^{f} & \boldsymbol{b}_{12} \boldsymbol{y}_{g y r, f}^{f} & \boldsymbol{b}_{13} \boldsymbol{y}_{g y r, f}^{f} & \boldsymbol{b}_{14} \boldsymbol{y}_{g y r, f}^{f} \\
\boldsymbol{b}_{21} \boldsymbol{y}_{g y r, f}^{f} & \boldsymbol{b}_{22} \boldsymbol{y}_{g y r, f}^{f} & \boldsymbol{b}_{23} \boldsymbol{y}_{g y r, f}^{f} & \boldsymbol{b}_{24} \boldsymbol{y}_{g y r, f}^{f} \\
\boldsymbol{b}_{31} \boldsymbol{y}_{g y r, f}^{f} & \boldsymbol{b}_{32} \boldsymbol{y}_{g y r, f}^{f} & \boldsymbol{b}_{33} \boldsymbol{y}_{g y r, f}^{f} & \boldsymbol{b}_{34} \boldsymbol{y}_{g y r, f}^{f} \\
q_{0}^{f} & q_{1} & q_{2} & q_{3}^{f}
\end{array}\right]
\end{aligned}
$$




$$
\left\{\begin{array}{cc}
\boldsymbol{b}_{11}=\left[\begin{array}{lll}
q_{0} & q_{3} & -q_{2}
\end{array}\right] & \boldsymbol{b}_{12}=\left[\begin{array}{lll}
q_{1} & q_{2} & q_{3}
\end{array}\right] \\
\boldsymbol{b}_{13}=\left[\begin{array}{lll}
-q_{2} & q_{1} & -q_{0}
\end{array}\right] & \boldsymbol{b}_{14}=\left[\begin{array}{lll}
-q_{3} & q_{0} & q_{1}
\end{array}\right] \\
\boldsymbol{b}_{21}=\left[\begin{array}{lll}
-q_{3} & q_{0} & q_{1}
\end{array}\right] & \boldsymbol{b}_{22}=\left[\begin{array}{lll}
q_{2} & -q_{1} & q_{0}
\end{array}\right] \\
\boldsymbol{b}_{23}=\left[\begin{array}{lll}
q_{1} & q_{2} & q_{3}
\end{array}\right] & \boldsymbol{b}_{24}=\left[\begin{array}{lll}
-q_{0} & -q_{3} & q_{2}
\end{array}\right] \\
\boldsymbol{b}_{31}=\left[\begin{array}{lll}
q_{2} & -q_{1} & q_{0}
\end{array}\right] & \boldsymbol{b}_{32}=\left[\begin{array}{lll}
q_{3} & -q_{0} & -q_{1}
\end{array}\right] \\
\boldsymbol{b}_{33}=\left[\begin{array}{lll}
q_{0} & q_{3} & -q_{2}
\end{array}\right] & \boldsymbol{b}_{34}=\left[\begin{array}{lll}
q_{1} & q_{2} & q_{3}
\end{array}\right]
\end{array}\right.
$$


CHAPTER 6

\section{Estimate Hand-finger position with one magnetometer and known relative orientation}

This chapter is published as a journal article: Yang, Z., Yan, S., Van Beijnum, B. J. F., Li, B., \& Veltink, P. H. (2021). Estimate Hand-Finger Position With One Magnetometer and Known Relative Orientation. IEEE Transactions on Instrumentation and Measurement, 70, 1-11. 


\title{
Estimate hand-finger position with one magnetometer and known relative orientation
}

\begin{abstract}
Inertial-sensor-based hand motion tracking has become a well-accepted method in many clinical applications including rehabilitation of the upper extremity etc. However, major drawbacks are that a sensor on each segment and kinematic chain rule are required. Thus, errors accumulate through the kinematic chain rule. Also, the length of each segment needs to be measured for each user.

We propose a novel method in which a permanent magnet on the dorsal side of the hand combined with an inertial sensor and a magnetometer (IMMU) on the fingertip are used to estimate the position of the thumb and index fingertip relative to the position of the dorsal side of the hand. The biggest advantages of the proposed method are that no prior information and kinematic chain rule are needed. Besides, the required number of sensors is low. A Levenberg-Marquardt (L-M) approach is presented to estimate the relative positions with the known orientations. The performance is demonstrated in multiple experiments in which various movement tasks were performed. The most complex task in which participants performed reaching and grasping movements based on action research arm test (ARAT) resulted in median distance error between thumb and index finger of $9.6 \%$.
\end{abstract}




\subsection{Introduction}

Hand-finger motion tracking has many useful applications including augmented reality (AR), virtual reality (VR) and especially physical rehabilitation $[129,130,132]$. Stroke patients or people who suffer from spinal cord injury need rehabilitation to regain full or partial functional recovery, including hand and finger dexterity [154]. The state of hand recovery can be determined by evaluating the relative motion between hands and fingers, such as the range of motion (ROM) of the hand, speed and accuracy [155]. Currently, the relative motion is usually assessed by the doctors and therapists in the hospital with certain standard movements such as the Fugl-Meyer or Jebsen-Taylor hand function assessment $[134,135]$, which are inaccurate and subjective. In order to make the evaluation more accurate and objective, optical tracking systems (OTSs) and inertial measurement unit (IMU) based systems including 3D accelerometers and 3D gyroscopes are commonly adopted $[156,157,158]$. When a $3 \mathrm{D}$ magnetometer is combined with an IMU, the unit is also referred to an IMMU (Inertial and Magnetic Measurement Unit). OTSs such as Vicon motion systems have a high position accuracy and are often used as reference systems in laboratory environments. However, these systems are expensive, require time-consuming placement of markers on subjects and have 'line of sight' problems. These systems measure position directly, orientation is measured indirectly $[159,160]$. In contrast, tracking systems based on IMMUs are inexpensive, have no 'line of sight' problem and directly measure angular velocity, acceleration and magnetic field. More importantly, they are more portable and can be applied outside the laboratory, which is promising for daily and long term monitoring at home [102, 145]. However, it cannot measure positions directly.

The IMMU-based system is often used to estimate the orientation in $3 \mathrm{D}$ space. One of the biggest challenges is to solve the drift issue. The change of orientation over time can be estimated by integrating gyroscope data. Nevertheless, this does not provide an orientation with respect to the global coordinate system and may drift. The drift can be compensated by the local gravity and geomagnetic field measured by the accelerometer and magnetometer $[101,40]$. The compensation can be quite effective when the magnetic disturbance and non-gravitational acceleration are small compared with the geomagnetic field and gravity. However, relative position estimation based on IMMUs is more difficult than the orientation estimation. Owing to inherent integration drift, position estimation based on strapdown integration cannot 
be performed with adequate accuracy for periods longer than a few seconds. To overcome this issue, biomechanical information and external position information are often applied. From the biomechanical information perspective, Kortier et al. proposed a method to reduce position drifts by exploiting a biomechanical model of the hand [102]. Subsequently, Salchow-Hömmen et al. improved the ability to resist magnetic disturbances by simplifying 3D rotational movements of segments into 2D rotational movements [26].

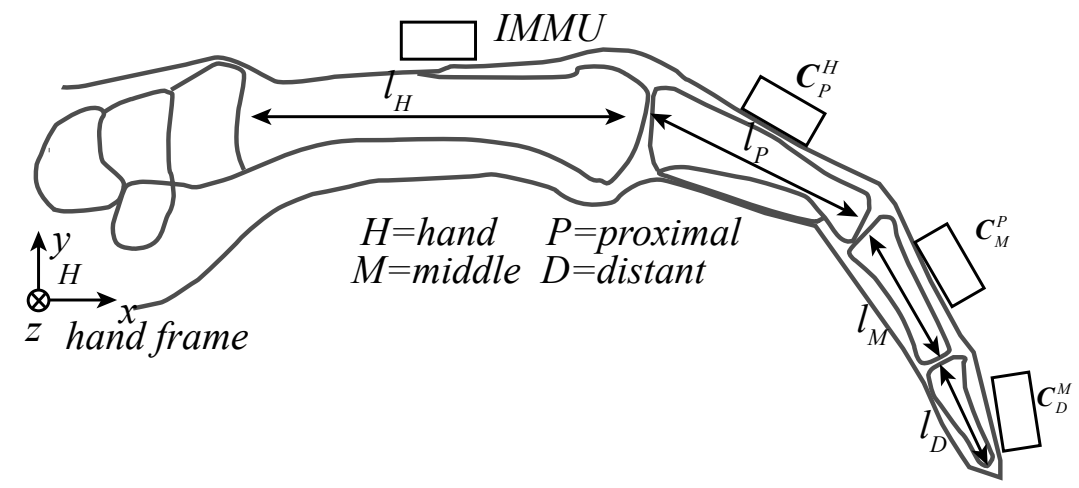

(a) Configuration of IMMUs on the hand with traditional methods

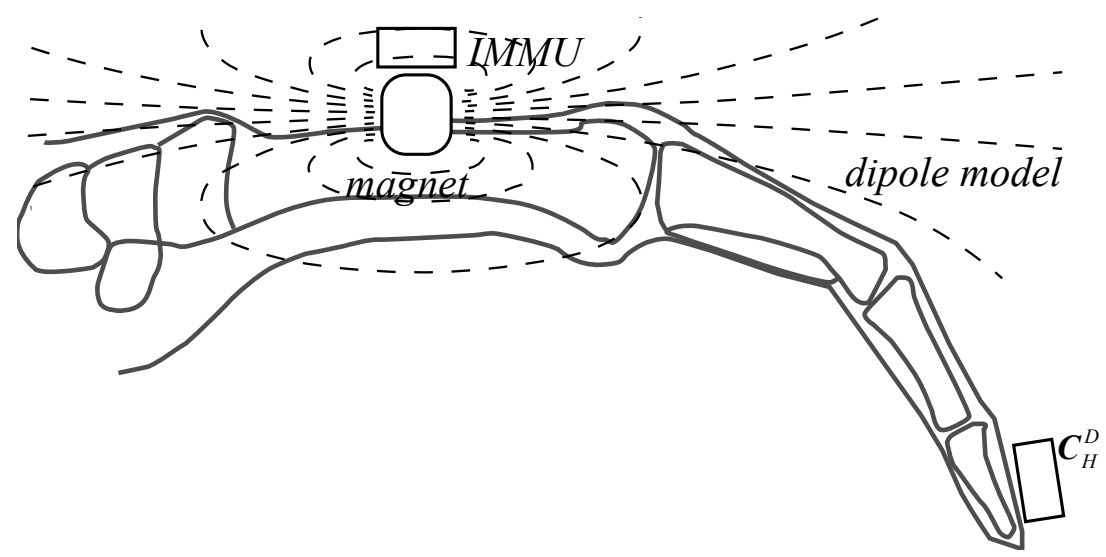

(b) Configuration of IMMUs on the hand with the proposed method

Figure 6.1: Comparison of the configurations between the traditional method and the proposed method

For the two methods above, the fingertip position was estimated with the biomechanical chain rule, as shown in Fig 6.1a,

$$
\boldsymbol{y}_{\text {tip,pos }}^{h}=\boldsymbol{l}_{H}^{H}+\boldsymbol{C}_{P}^{H}\left(\boldsymbol{l}_{P}^{P}+\boldsymbol{C}_{M}^{P}\left(\boldsymbol{l}_{M}^{M}+\boldsymbol{C}_{D}^{M} \boldsymbol{l}_{D}^{D}\right)\right)
$$


where $l_{i}^{i}(i=H, P, M, D)$ represents segment position in its own frame. $C_{i}^{j}(i, j=$ $H, P, M, D)$ represents relative orientation between segments. The relative orientation and segment lengths are required for using the biomechanical model. There are three major drawbacks: firstly, model and orientation errors accumulate through the kinematic chain, resulting in position errors. Secondly, the segment lengths vary among different people, and therefore need to be measured for every person before use, which brings error source and is troublesome for users. Thirdly, an IMU is located on each finger segment, which makes the system complex and should preferably be simplified.

In this paper, we propose to use a magnet on the hand as a passive magnetic source and one magnetometer on a fingertip of interest as a receiver to estimate the relative position, given known relative orientation between hands and fingers. There are numerous advantages of using such a passive magnetic localization system: firstly, there is no need to add additional sensors, since magnetometers are embedded in available IMMUs and can be used as receivers. Secondly, it does not consume power. Thirdly, the intermediate IMMUs are neglected which makes the volume smaller and more convenient for users. The relative orientation can be estimated by only exploiting IMUs, placed on the dorsal side of the hand and the fingertips of interest, which can be found in our previous research [161]. Or, the relative orientation can also be estimated with only IMUs if we assume finger movement as 2DOF [162, 149], such as index finger movements. The aim of this study is to investigate the possibility of estimating relative positions between the hand and several fingertips including thumb and index finger using one magnet on the hand and a single magnetometer on each fingertip of interest, assuming their relative orientations are known. Thumb and index finger are chosen since they play a more important role compared with rest fingers during daily tasks such as grasp and pinch [163, 164]. This goal is achieved by analyzing the potentially estimated positions with a dipole model and assuming the magnetic field generated by the magnet is much stronger than the geomagnetic field.

\subsection{Methods}

The flowchart of the proposed method is shown in Fig 6.2. The magnet on the fingertip is assumed as a magnetic dipole. When the relative orientation between the hand and fingertip is known, the magnetic field induced by 
the magnet can be transformed from sensor frame to magnet frame. Subsequently, two possible position estimations are obtained. Through a ghost solution elimination method, the fingertip position relative to the hand is obtained. Finally, the results are compared with the position reference from an OTS.

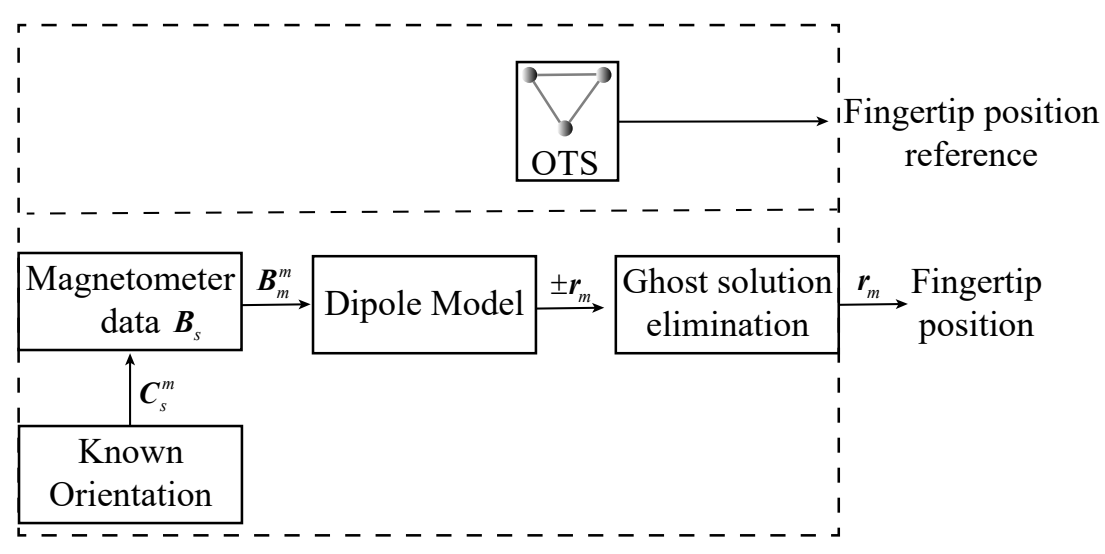

Figure 6.2: Flowchart of the proposed method.

\subsubsection{The magnetic source and sensor model}

When a ferromagnetic object is far from the sensor, more than 2.5 times of its length, the object can be approximated as a dipole [43, 165]. As shown in Fig 6.3, the magnetic field at $\boldsymbol{r}_{m}$ generated by the magnet (located at the origin) is

$$
\boldsymbol{B}_{m}^{m}=\frac{\mu_{0} M_{m}}{4 \pi r_{m}^{3}}\left[\frac{3}{2} \sin 2 \theta \boldsymbol{e}_{\rho}+\left(3 \cos ^{2} \theta-1\right) \boldsymbol{e}_{z}\right]
$$

where $\mu_{0}$ is the permeability of free space and $M_{m}$ is the magnetic moment. $\boldsymbol{r}_{m}$ is the position in the magnet frame, expressed as follows in a spherical coordinate system:

$$
r_{m, x}=r_{m} \cos \theta \sin \varphi, r_{m, y}=r_{m} \cos \theta \cos \varphi, r_{m, z}=r_{m} \sin \theta
$$

where $\left(r_{m}, \theta, \varphi\right)$ gives the radial distance, polar angle, and azimuthal angle. $\boldsymbol{e}_{\rho}$ and $\boldsymbol{e}_{z}$ are projected unit vectors of $\boldsymbol{r}_{m}$ on the horizontal plane and vertical axis, as shown in Fig 6.3. 


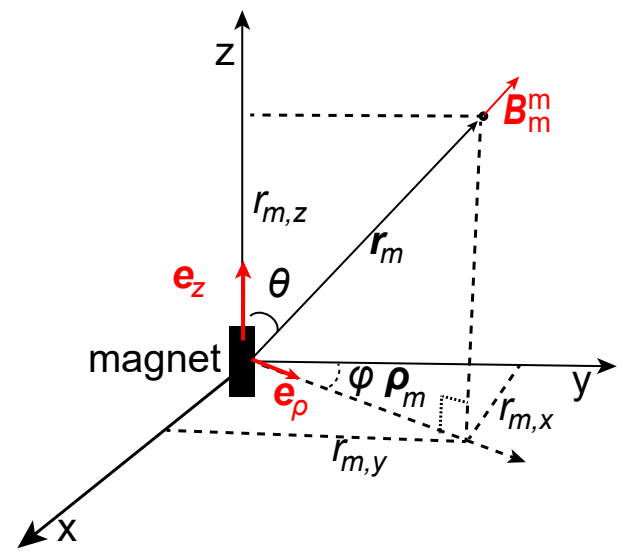

Figure 6.3: Dipole model of a magnet. The coordinate system is centered at the center of the magnet, and the z-axis is chosen along the magnetic axis. The magnetic field at position $\boldsymbol{r}_{m}$ is $\boldsymbol{B}_{m}$.

When the magnetic field at position $\boldsymbol{r}_{m}$ is measured by a three-axis magnetometer (TAM), let it be denoted as $\boldsymbol{y}_{\text {mag }}^{s}$, then this measurement can be modeled as:

$$
\boldsymbol{y}_{\text {mag }}^{s}=\boldsymbol{A}\left(\boldsymbol{B}_{m}^{s}+\boldsymbol{B}_{e}^{s}+\boldsymbol{b}\right)+\boldsymbol{n}_{B}
$$

where $\boldsymbol{A}$ is the integrated error parameter including sensitivity errors, nonorthogonality errors and soft-iron effect errors [166]. $\boldsymbol{b}$ is the integrated error including offset errors and hard-iron effect errors. $\boldsymbol{B}_{m}^{s}$ is the magnetic field generated by the magnet. $\boldsymbol{B}_{e}^{s}$ is the disturbing magnetic field including geomagnetic field and surrounding disturbance, and $\boldsymbol{n}_{B}$ is the measurement noise. $\boldsymbol{A}$ and $\boldsymbol{b}$ can be estimated by calibration procedures [167, 168]. When $\left|\boldsymbol{B}_{m}^{s}\right|$ is much stronger than $\left|\boldsymbol{B}_{e}^{s}\right|, \mathrm{Eq}(6.4)$ can be approximated as

$$
\boldsymbol{y}_{m a g}^{s} \approx \boldsymbol{A}\left(\boldsymbol{B}_{m}^{s}+\boldsymbol{b}\right)+\boldsymbol{n}_{B}
$$

\subsubsection{Relations between two segments}

We place the magnet and magnetometer on two different segments, as shown in Fig 6.4. The magnetic field generated by the magnet in the magnet frame can be obtained as

$$
\boldsymbol{B}_{m}^{m} \approx \boldsymbol{C}_{s}^{m}\left(\boldsymbol{A}^{-1} \boldsymbol{y}_{m a g}^{s}-\boldsymbol{b}\right)=\boldsymbol{C}_{B_{1}}^{m} \boldsymbol{C}_{B_{2}}^{B_{1}} \boldsymbol{C}_{s}^{B_{2}}\left(\boldsymbol{A}^{-1} \boldsymbol{y}_{m a g}^{s}-\boldsymbol{b}\right)
$$


where $C_{B_{1}}^{m}$ is the rotation matrix from the segment $B_{1}$ to the magnet, $C_{s}^{B_{2}}$ is the rotation matrix from the magnetometer to the segment $B_{2} . C_{B_{1}}^{m}$ and $C_{s}^{B_{2}}$ can be obtained from sensor to segment calibration. $C_{B_{2}}^{B_{1}}$ is the rotation matrix between two segments. The position of the magnet in the frame of

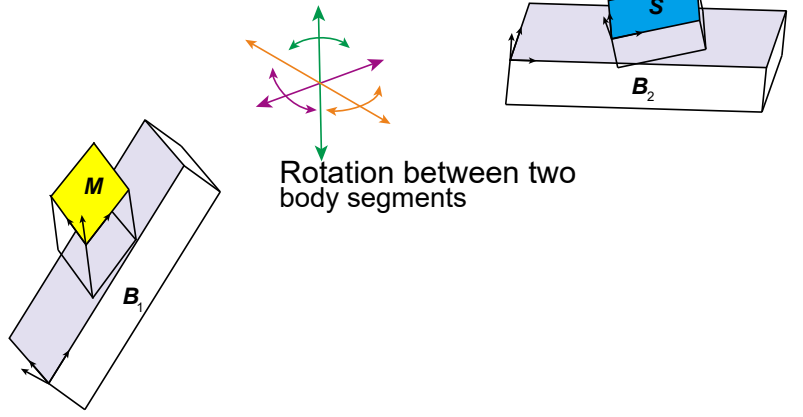

Figure 6.4: Relations between two segments. $B_{1}$ and $B_{2}$ represent two body segments. $M$ represents the magnet, s represents the magnetometer.

segment $B_{1}$ is obtained by

$$
\boldsymbol{r}_{B_{1}}=C_{m}^{B 1} \boldsymbol{r}_{m}
$$

\subsubsection{Relative position estimation}

\section{Existence of position estimation}

When orientation $\boldsymbol{C}_{m}^{s}$ is known, $\boldsymbol{B}_{m}^{m}$ can be obtained based on Eq (6.6). Subsequently, $\boldsymbol{r}_{m}$ can be estimated base on $\boldsymbol{B}_{m}^{m}$ and Eq (6.2). Possible estimations can be categorized into two cases: when $\boldsymbol{M}_{m}$ is perpendicular to $\boldsymbol{r}_{m}$, possible solutions are distributed on a circle in 3D space, as shown in Fig 6.5b. Otherwise, two possible solutions can be obtained, as shown in Fig 6.5c. The proof can be found in Appendix C.1.

\section{Estimation of $r_{m}$ and $\theta$}

When the orientation $\boldsymbol{C}_{m}^{s}$ is known, the measurement output can be rotated to the magnet frame according to $\mathrm{Eq}(6.5)$. Then $\boldsymbol{B}_{m}^{m}$ is known based on Eq (6.5)

$$
\boldsymbol{B}_{m}^{m}=\left(\boldsymbol{C}_{m}^{s}\right)^{-1}\left(\boldsymbol{A}^{-1} \boldsymbol{y}_{\text {mag }}^{s}-\boldsymbol{b}\right)
$$

Subsequently, the relative position $\boldsymbol{r}_{m}$ with known relative orientation $\boldsymbol{C}_{m}^{S}$ and $\boldsymbol{B}_{m}^{m}$ can be estimated by solving a nonlinear optimization problem based 


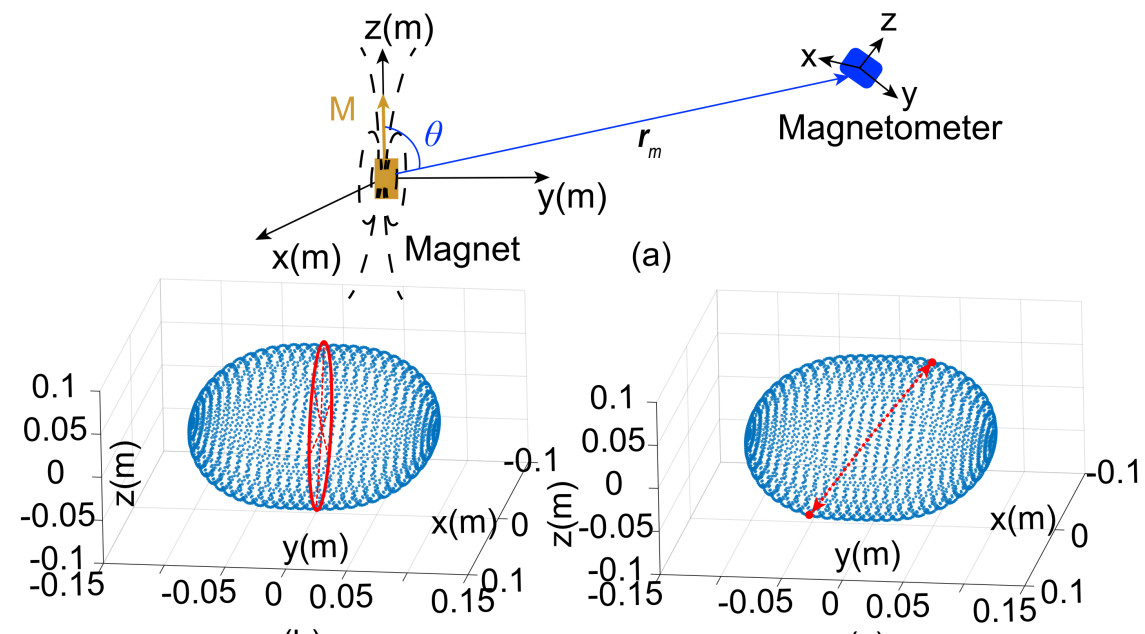

(b)

(c)

Figure 6.5: Illustration of the position estimation with one TAM. $\boldsymbol{M}_{m}$ is set as $[0,0,1] A \cdot m^{2}$. (a) shows a magnet in the center of a coordinate system, with the direction along $z$ axis. (b) and (c) show the possible positions with known orientation $\boldsymbol{C}_{m}^{s}$. For $(b), \boldsymbol{r}_{m} \perp \boldsymbol{M}_{m}$, possible positions are distributed on a circle in $3 D$ space. For (c), $\boldsymbol{r}_{m}$ is not perpendicular to $\boldsymbol{M}_{m}$, two possible positions can be estimated as $\pm \boldsymbol{r}_{m}$.

on $\mathrm{Eq}(6.2)$.

$$
\left\{\begin{array}{c}
\left(r_{m}, \theta\right)=\operatorname{argmin}\|\boldsymbol{g}\|_{2}^{2} \\
\boldsymbol{g}=f\left(r_{m}, \theta\right)-\left(B_{m, h}^{m}, B_{m, z}^{m}\right)
\end{array}\right.
$$

where $B_{m, h}^{m}$ and $B_{m, z}^{m}$ arethe horizontal and vertical strength of $\boldsymbol{B}_{m}^{m}$

$$
B_{m, h}^{m}=\sqrt{\left(B_{m, x}^{m}\right)^{2}+\left(B_{m, y}^{m}\right)^{2}}
$$

$f\left(r_{m}, \theta\right)$ is obtained based on $\mathrm{Eq}(6.2)$

$$
f\left(r_{m}, \theta\right)=\frac{\mu_{0} M_{m}}{4 \pi r_{m}^{3}}\left[\frac{3}{2} \sin 2 \theta \boldsymbol{e}_{\rho}+\left(3 \cos ^{2} \theta-1\right) \boldsymbol{e}_{z}\right]
$$

Then Eq (6.9) can be solved by the Levenberg-Marquardt (L-M) method. After initialization for $\left(r_{m}, \theta\right)^{(0)}$, the update can be done using the following equation:

$$
\left(r_{m}, \boldsymbol{\theta}\right)^{(k+1)}=\left(r_{m}, \boldsymbol{\theta}\right)^{(k)}-\left(\lambda \boldsymbol{I}_{2 \times 2}+\boldsymbol{J}_{\boldsymbol{g}}^{T} \boldsymbol{J}_{\boldsymbol{g}}\right)^{-1} \boldsymbol{J}_{\boldsymbol{g}}^{T} \boldsymbol{g}
$$


where $\boldsymbol{I}_{2 \times 2}$ is an unit matrix and $\lambda$ is a damping parameter that would change automatically by L-M method. When $\lambda$ is small, its performance is close to the Gauss-Newton method. Otherwise, its performance is close to the gradient-descent method. With the L-M method, there is a greater possibility of convergence when we have a bad initial value. $\boldsymbol{J}_{\boldsymbol{g}}$ is a jacobian matrix of $g$ :

$$
\boldsymbol{J}_{\boldsymbol{g}}=\frac{\partial \boldsymbol{f}}{\partial\left(r_{m}, \boldsymbol{\theta}\right)_{m}}=\left[\begin{array}{ll}
\frac{\partial f\left(\left(r_{m}, \theta\right)\right)}{\partial r_{m}} & \frac{\partial f\left(\left(r_{m}, \theta\right)\right)}{\partial \theta}
\end{array}\right]
$$

where

$$
\left\{\begin{array}{c}
\frac{\partial f\left(r_{m}, \theta\right)}{\partial r_{m}}=\frac{-3 \mu_{0} M_{m}}{4 \pi r_{m}^{4}}\left[\frac{3}{2} \sin 2 \theta \boldsymbol{e}_{\rho}+\left(3 \cos ^{2} \theta-1\right) \boldsymbol{e}_{z}\right] \\
\frac{\partial f\left(r_{m}, \theta\right)}{\partial \theta}=\frac{\mu_{0} M_{m}}{4 \pi r_{m}^{3}}\left[3 \cos 2 \theta \boldsymbol{e}_{\rho}-(3 \sin 2 \theta) \boldsymbol{e}_{z}\right]
\end{array}\right.
$$

\section{Estimation of $\varphi$}

In a cartesian coordinate system, Eq (6.2) can be rewritten as

$$
\boldsymbol{B}_{m}^{m}=\frac{\mu_{0}}{4 \pi}\left(\frac{3 \boldsymbol{r}_{m}^{T} \boldsymbol{M}_{m} \boldsymbol{r}_{m}}{r_{m}^{5}}-\frac{\boldsymbol{M}_{m}}{r_{m}^{3}}\right)
$$

As shown in Fig 6.3, the direction of magnet is along $\mathrm{z}$-axis in magnet frame, $\boldsymbol{M}_{m}=\left[\begin{array}{lll}0 & 0 & M_{m}\end{array}\right]$. Eq (6.15) can be simplified as

$$
\boldsymbol{B}_{m}^{m}=\frac{\mu_{0} M_{m}}{4 \pi r^{5}}\left[\begin{array}{lll}
r_{m, x} r_{m, z} & r_{m, y} r_{m, z} & r_{m, z}^{2}-r_{m}^{2}
\end{array}\right]
$$

Based on Eq (6.3) and (6.16), $\varphi$ can be obtained

$$
\tan \varphi=\frac{r_{m, y}}{r_{m, x}}=\frac{B_{m, y}}{B_{m, x}}
$$

\section{Ghost solution elimination}

From the previous subsection, $\boldsymbol{r}_{m}$ can be estimated either on a circle or two points with additional information $\boldsymbol{C}_{m}^{s} . \boldsymbol{r}_{m}$ lies on a circle only happens when $\theta=\pi / 2$, which very rarely happens and can be prevented by designing the magnet configuration. The goal of this subsection is to determine solution $\boldsymbol{r}_{m}$ from possible solutions $\pm \boldsymbol{r}_{m}$. For the daily task, positions of the index and thumb fingertips are restricted

$$
\boldsymbol{r}_{f x}>0
$$




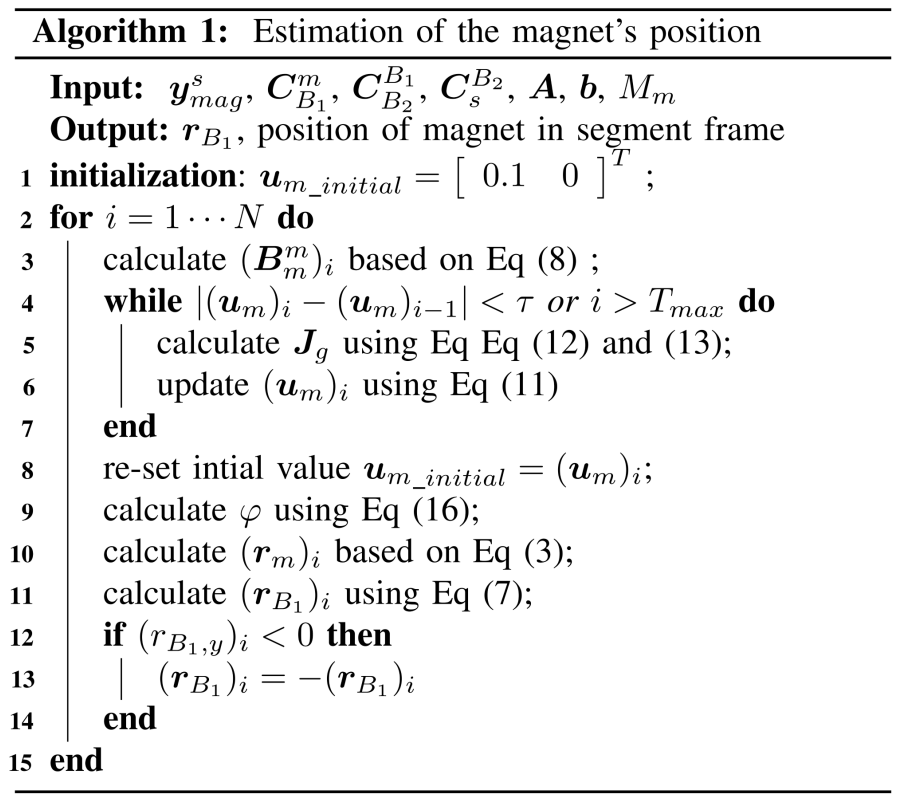

where $\boldsymbol{r}_{f x}$ is the position of fingers along $\mathrm{x}$-axis of the hand frame (see Fig 6.7 where this direction is defined). f represents fingers, including the thumb and index finger. Then, $\boldsymbol{r}_{m}$ is uniquely determined. The algorithm proposed and derived above is summarized as Algorithm 1.

\subsubsection{Uncertainty of the estimation}

As shown in Eq (6.4), the magnetic field generated by the magnet is disturbed by the geomagnetic field, ferromagnetic object and measurement noise. The sensitivities of estimations to the disturbances vary at different positions, leading to different uncertainties at different positions. Suppose we have a magnetic disturbance $\boldsymbol{B}_{e}^{m}$ (expressed with $\boldsymbol{e}_{\rho}$ and $\boldsymbol{e}_{z}$ ), then an error $\delta \boldsymbol{u}_{m}\left(\delta r_{m}, \delta \theta\right)$ caused by the $\boldsymbol{B}_{e}^{m}$ will be added to the relative position $\boldsymbol{u}_{m}\left(r_{m}, \theta\right)$. Then we can obtain the following equation based on Eq (6.11).

$$
\left\{\begin{array}{c}
\boldsymbol{y}_{\text {mag }}^{m} \approx f\left(\boldsymbol{u}_{m}\right) \\
\boldsymbol{y}_{m a g}^{m}+\boldsymbol{B}_{e}^{m}
\end{array}\right.
$$

Based on Eq (6.19) and Eq (6.13), we obtain :

$$
\begin{aligned}
\boldsymbol{B}_{e}^{m} & =f\left(\boldsymbol{u}_{m}+\delta \boldsymbol{u}_{m}\right)-f\left(\boldsymbol{u}_{m}\right) \\
& \approx \boldsymbol{J}_{g} \delta \boldsymbol{u}_{m}
\end{aligned}
$$


The relative position error $\delta \boldsymbol{u}_{m}$ caused by the disturbance $\boldsymbol{B}_{e}^{m}$ can be approximated as

$$
\delta \boldsymbol{u}_{m} \approx \boldsymbol{J}_{g}^{-1} \boldsymbol{B}_{e}^{m}
$$

According to the error propagation theory, the variance $\boldsymbol{Q}$ of $\delta \boldsymbol{u}_{m}$ can be expressed as

$$
\boldsymbol{Q}=\operatorname{Cov}\left(\delta r_{m}, \delta \theta\right)=\boldsymbol{J}_{g}^{-1} \operatorname{Var}\left(\boldsymbol{B}_{e}^{m}\right)\left(\boldsymbol{J}_{g}^{-1}\right)^{T}
$$

where $\operatorname{Var}\left(\boldsymbol{B}_{e}^{m}\right)$ is the variance of disturbance $\boldsymbol{B}_{e}^{m} \cdot \boldsymbol{Q}$ is a $2 \times 2$ matrix which represents the uncertainty ellipse of the estimation at position $\boldsymbol{u}_{m}$. Fig 6.6 shows a simulation result that the uncertainty region caused by a Gaussian disturbance with zero mean and standard deviation $50 \mu \mathrm{T}$. With a certain $\theta$, the uncertainty increases as $r_{m}$ increases. With a fixed distance $r_{m}$, the uncertainty is smaller when $\theta$ approximates $0^{\circ}$ and larger when $\theta$ approximates $90^{\circ}$.

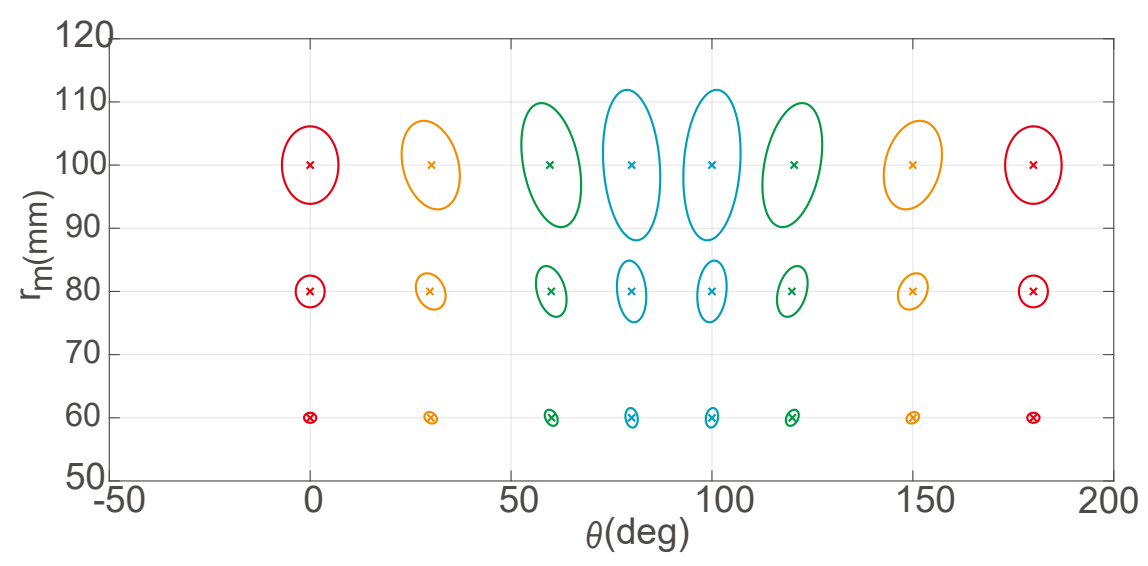

Figure 6.6: Simulation of the estimated uncertainty with respect to $r_{m}$ and $\theta$. $\boldsymbol{M}_{m}=\left[\begin{array}{lll}0 & 0 & 1.6\end{array}\right] \mathrm{A} \cdot \mathrm{m}^{2}, r_{m}=60 \mathrm{~mm}, 80 \mathrm{~mm}, 100 \mathrm{~mm} . \theta=$ $0^{\circ}, 30^{\circ}, 60^{\circ}, 80^{\circ}, 100^{\circ}, 120^{\circ}, 150^{\circ}, 180^{\circ} . \varphi=0^{\circ}$. The magnetic disturbance is set as a Gaussian distribution with zero mean and $50 \mu T$ standard deviation. 


\subsection{Experimental methods}

\subsubsection{Experiment setups}

The sensor system included 3 IMMUs ( MPU9250, InvenSense) fixed on the most distal segment of the thumb, index finger and the dorsal side of the hand. A magnet was also fixed on the dorsal side of the hand, as shown in Fig 6.7. The orientation and strength of the magnetic moment needs to be optimized in order to minimize the overall position estimation error. Based on the simulation results, the best option for $\theta$ (angle between $\boldsymbol{r}_{m}$ and $\boldsymbol{M}_{m}$ ) should be between $0^{\circ}$ and $90^{\circ}$ during flexion and extension of the thumb and index finger. As shown in Fig 6.7, the angle between $\boldsymbol{M}_{m}$ and $x$ axis is denoted as $\alpha$, which was set as $45^{\circ}$. The strength of the magnet is $3.2 \mathrm{~A} \cdot \mathrm{m}^{2}$. The TAM and accelerometer were sampled synchronously at $100 \mathrm{~Hz}$. All the data was transmitted to a PC through a USB connection. A Vicon system with 8 cameras was used and the data was preprocessed with software Vicon Nexus 2.10.2. On each IMMU, a cluster of 3 markers were placed as shown in Fig 6.7, which were used to obtain the relative orientation between the hand and fingers as known orientation $\boldsymbol{C}_{m}^{s}$. The Vicon system also provided the relative position reference for $\boldsymbol{r}_{m}$. The sample frequency of Vicon system was $100 \mathrm{~Hz}$.

\subsubsection{Orientation from the OTS}

The known orientation is obtained from clusters of markers. For each cluster, we obtain two vectors $\boldsymbol{r}_{1}, \boldsymbol{r}_{2}$ spanning a configuration of three markers, as shown in Fig 6.8. Then the orientation of the marker frame can be calculated as:

$$
\boldsymbol{C}_{m}^{g}=\left[\begin{array}{lll}
\frac{\boldsymbol{n}_{\boldsymbol{r}_{2}} \times \boldsymbol{n}_{\boldsymbol{r}_{3}}}{\left|\boldsymbol{n}_{\boldsymbol{r}_{2}} \times \boldsymbol{n}_{\boldsymbol{r}_{3} \mid}\right|} & \boldsymbol{n}_{\boldsymbol{r}_{2}} & \boldsymbol{n}_{\boldsymbol{r}_{3}}
\end{array}\right]
$$

where $\boldsymbol{n}_{\boldsymbol{r}_{1}}$ and $\boldsymbol{n}_{\boldsymbol{r}_{2}}$ are normalized vectors corresponding to $\boldsymbol{r}_{1}$ and $\boldsymbol{r}_{2}$ respectively. $\boldsymbol{n}_{\boldsymbol{r}_{3}}=\boldsymbol{n}_{\boldsymbol{r}_{1}} \times \boldsymbol{n}_{\boldsymbol{r}_{2}} /\left|\boldsymbol{n}_{\boldsymbol{r}_{1}} \times \boldsymbol{n}_{\boldsymbol{r}_{2}}\right|$. Through the rotation matrix between the OTS coordinate frame and the sensor frame, the orientation assessed by the OTS can be easily transferred to the sensor frame. 


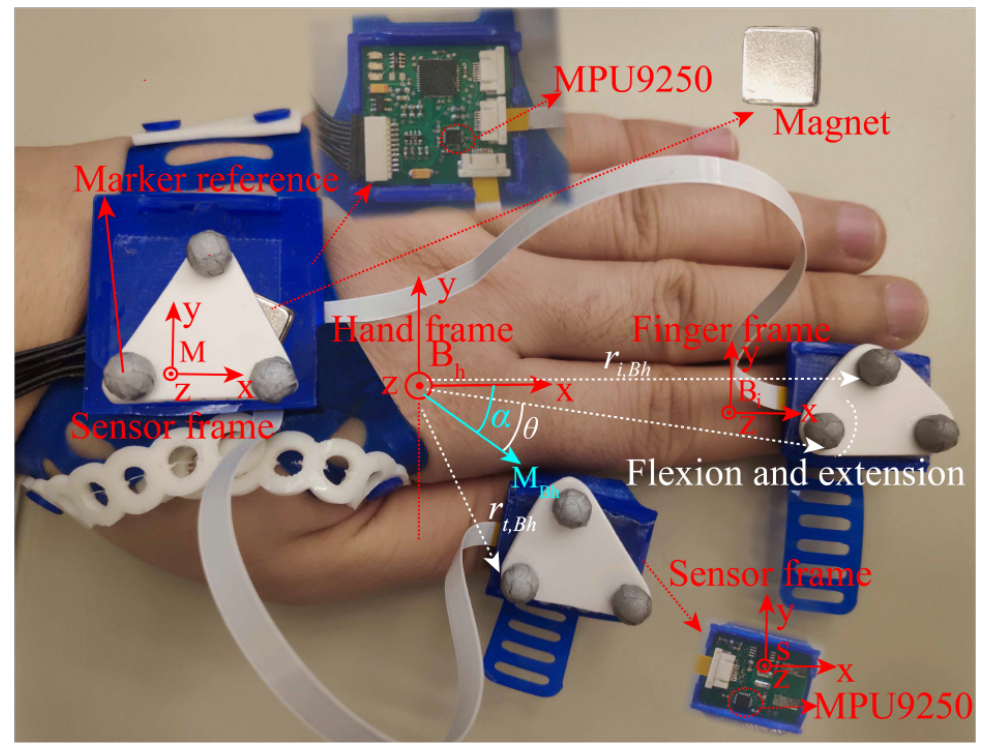

Figure 6.7: Experiment setup. Three measurement units were attached to the dorsal side of the hand, index fingertip and thumb fingertip. A magnet was used as the a passive magnetic source, its size was $11 \mathrm{~mm} \times 11 \mathrm{~mm} \times 11 \mathrm{~mm}$. Three clusters of markers were attached on the surfaces of the measurement setups and the magnet.

\subsubsection{Synchronization and alignment between the TAM and OTS}

The TAM and OTS were two independent systems, which needed to be synchronized. In addition, the OTS provided known relative orientations for the TAM, and therefore needed to be aligned. Here, a gyroscope was used as an intermediate to synchronize and align two systems. The process is shown in Fig 6.8.

We obtained angular velocities from the OTS and gyroscope but in different coordinate frames. The synchronization and alignment were achieved by correlating angular velocities in two different coordinate frames. The detailed algorithm can be found in Appendix C.2.

Since the center of the marker did not coincide with the center of the magnet and TAM, the relative position between the marker and the magnet, as well as the relative position between the marker and TAM were estimated 


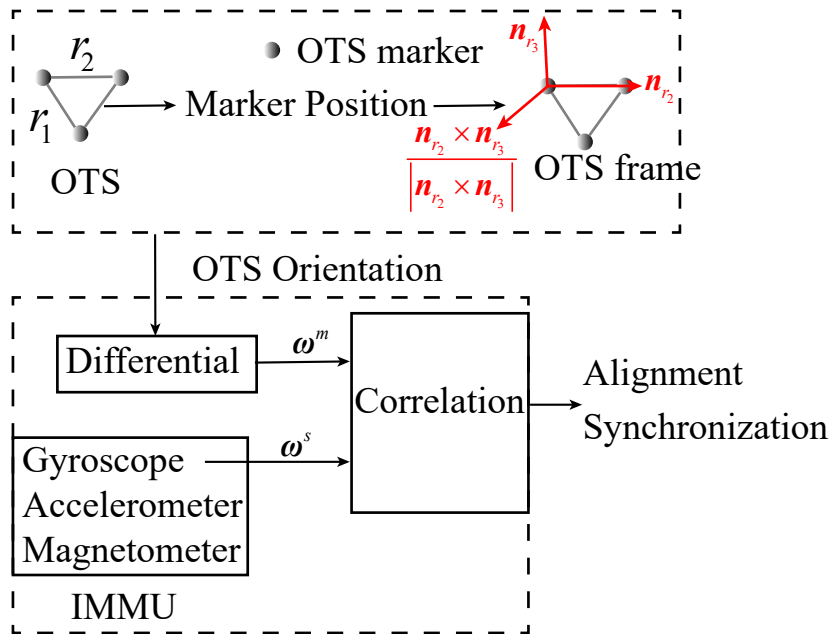

Figure 6.8: Alignment and synchronization of the OTS and IMMU system. Angular velocities can be obtained from OTS-based orientation, which can also be measured by the IMMU. The alignment and synchronization were achieved by correlating angular velocities from different frames. The gyroscope was assumed to align with the magnetometer, which can be achieved by calibrations.

from the experimental photos.

\subsubsection{Protocols}

The performance of the proposed method was evaluated for different movements (flexion and extension of index finger and thumb, abduction and adduction and pinch), functional tasks (move object from position A to position $\mathrm{B}, \mathrm{C}$ and D) and disturbances (geomagnetic disturbance and ferromagnetic object disturbance). See Figure 6.9 for the illustration of these experimental cases. Functional tasks are designed based on action research arm test (ARAT). The experimental protocol consists of a calibration measurement phase followed by 5 experiments, which are described in the following subsections.

\section{Sensor to segment calibration}

Gyroscopes and accelerometers were used to realize the sensor to segment calibration. The main steps of this procedure are as follows, more 


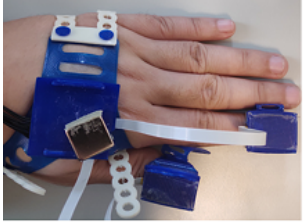

(a)

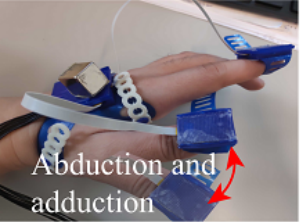

(e)

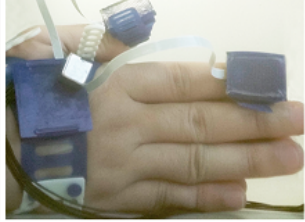

(b)

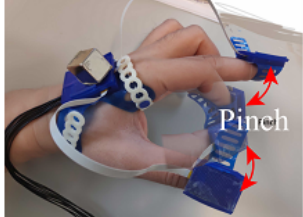

(f)

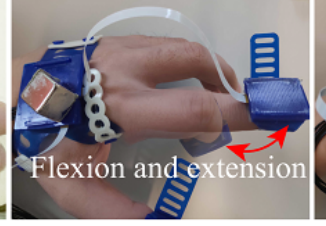

(c)

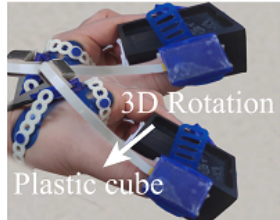

(g)

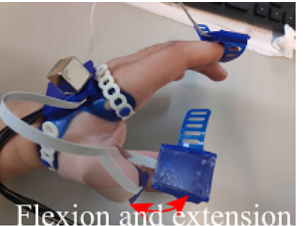

(d)

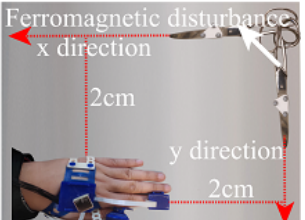

(h)

Figure 6.9: Illustration of the experimental cases. (a) Hand on the table flat. (b) Hand on the table vertically. (c) Index finger flexion and extension. (d) Thumb flexion and extension. (e) Thumb abduction and adduction. (f) Pinch. $(g)$ Hold a plastic cube and rotate in $3 D$ space, while the relative orientation between the hand and fingers remains unchanged. (h) Move the ferromagnetic object near the setup, while put the hand statically on the surface of the wooden table without any metal bars or screws.

details can be found in Kortier et al's work [102]:

(1) Put the hand flat on the table and static for $10 \mathrm{~s}$, with the back of the hand facing upward, as shown in Fig 6.9a;

(2) Put two hands together on the table vertically and hold for $10 \mathrm{~s}$, as shown in Fig 6.9b;

(3) Flex and extend the index finger repeatedly for $10 \mathrm{~s}$, as shown in Fig $6.9 \mathrm{c}$

(4) Flex and extend the thumb repeatedly for $10 \mathrm{~s}$, as shown in Fig 6.9d.

\section{Experiment 1-Joint movements}

(1) Stretch the arm with the elbow on the table and then flex and extend the index finger for $20 \mathrm{~s}$, at a rate of 3 times per second;

(2) Repeat (1), with the thumb;

(3) Abduct and adduct the thumb at least 3 times per second for $20 \mathrm{~s}$, as shown in Fig 6.9e;

(4) Perform a pinch movement with the thumb and index finger for $20 \mathrm{~s}$, at a rate of 3 times per second, as shown in Fig 6.9f. 


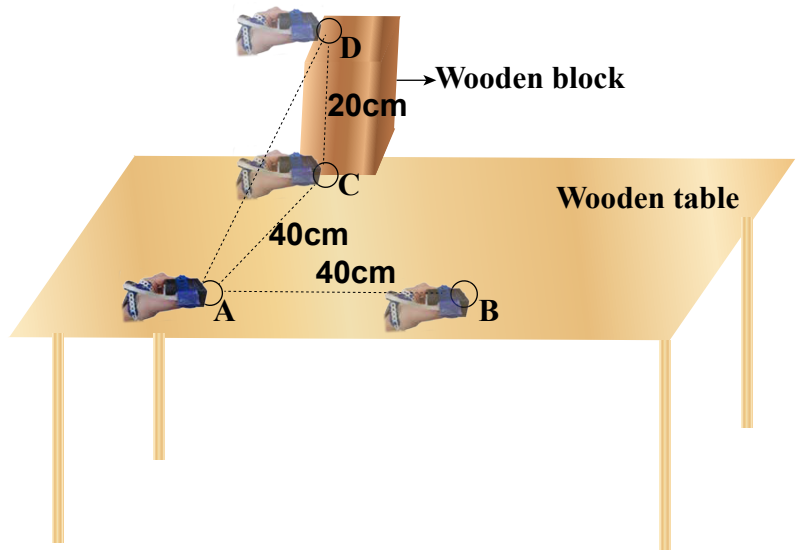

Figure 6.10: Illustration of the movements during functional tasks. The participant was asked to grasp the plastic cube from position A to position $B, C, D$ individually. The lengths of $A B$ and $A C$ are 40 $\mathrm{cm} . A B$ and $A C$ are on the surface of the table and they are perpendicular to each other. $C D$ is perpendicular to the surface of the table and $20 \mathrm{~cm}$ long.

\section{Experiment 2-Functional tasks}

(1) Put the palm flat on the table near position A for $10 \mathrm{~s}$, as shown in Fig 6.10;

(2) Grasp the rectangle object with tips of the index finger and thumb from the position $\mathrm{A}$ and put it to the position $\mathrm{B}$, then move the hand to the initial position, as shown in Fig 6.10;

(4) Repeat (1) and (2), but from the position A to C;

(5) Repeat (1) and (2), but from the position A to D;

\section{Experiment 3-Influence of geomagnetic disturbances}

Grasp the rectangle object, and rotate the object in 3D space randomly for $30 \mathrm{~s}$, as shown in Fig 6.9g. During the rotation, do not change the relative orientation and position between the hand and fingers.

\section{Experiment 4-Influence of ferromagnetic object disturbances}


(1) As shown in Fig 6.9h, put the palm flat on the table and keep it static for $10 \mathrm{~s}$;

(2) Hold a pair of scissors as a ferromagnetic object and move it along $\mathrm{x}$-axis and $\mathrm{y}$-axis of the hand frame. The hand frame is shown in Fig 6.7, the trajectories are shown in Fig 6.9h.

8 participants were involved in the experiment 2 and 3. One participant was involved in experiment 1, 4 and 5 since the performance of the algorithm was not related to the participants in these experiments. All the experimental data was processed based on Algorithm 1, the estimated relative position was compared with the Vicon system.

\subsection{Results}

\subsubsection{Experiment 1-Joint movements \& Experiment 2- Functional tasks}

Fig 6.11 shows the box plot of median error from 8 participants during different movements. Among joint movements, the median distance errors during index finger flexion and extension, thumb flexion and extension, thumb abduction and adduction and pinch movement are 4.9\%, 8.0\%, 8.7\% and 6.4\%. The median distance error during task 1 (move object A to B), task 2 (move object $\mathrm{A}$ to $\mathrm{C}$ ) and task 3 (move object $\mathrm{A}$ to $\mathrm{D}$ ) are $9.4 \%, 9.3 \%$ and $8.2 \%$. The discontinuity of the estimation based on OTS is caused by the 'line of sight' problem.

\subsubsection{Experiment 3-Influence of geomagnetic disturbances}

During the $3 \mathrm{D}$ rotational movements, there is no movements between hand and fingers, relative position errors caused by the changing geomagnetic field in the sensor frame are shown in Fig 6.12. Fingertip position based on the OTS was chosen as a position reference, whose standard deviation was $\left[\begin{array}{lll}0.53 \% & 0.65 \% & 0.53 \%\end{array}\right]$. The standard deviation of fingertip position errors based on magnetometer was $\left[\begin{array}{lll}3.3 \% & 3.9 \% & 2.8 \%\end{array}\right]$, which was caused by the geomagnetic disturbance. 

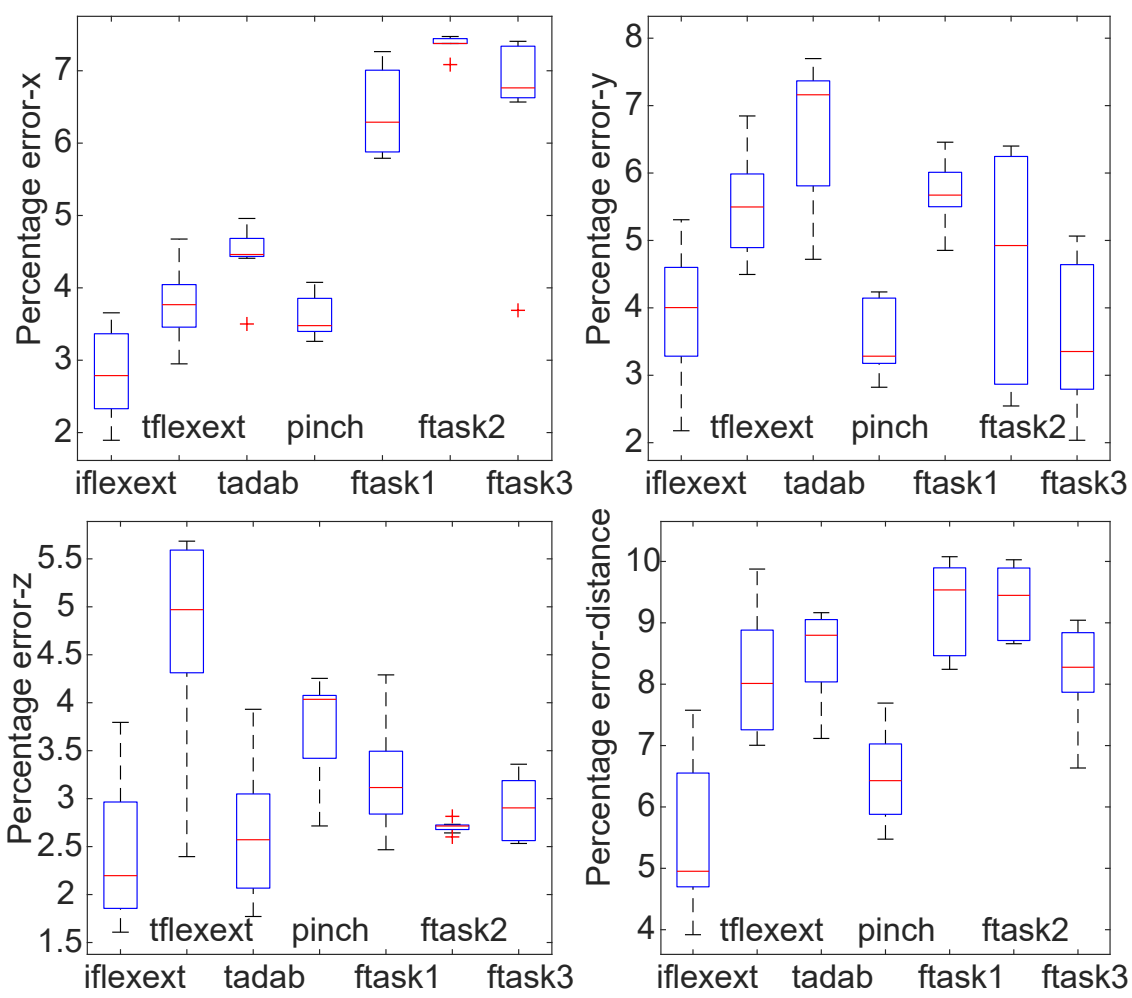

Figure 6.11: Relative position errors during different movements evaluated in 8 subjects. (a), (b), (c) represent relative position errors along $x, y, z$-axis in the hand frame. (d) represents the relative distance error. iflex, tflexext, tadab represent index finger flexion-extension, thumb flexion-extension and thumb abduction-adduction respectively. ftask 1 , ftask 2 and ftask 3 represent moving the object from $A$ to $B, C$ and $D$ respectively as defined in Experiment 3 (also see Fig 6.7). 

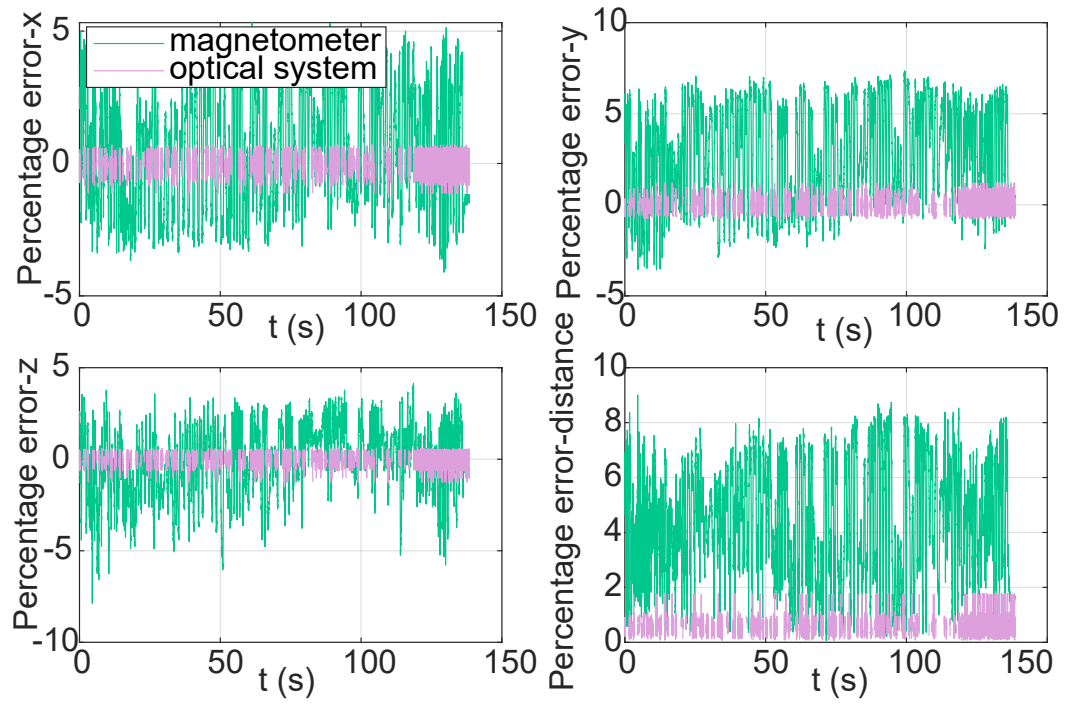

Figure 6.12: Relative position errors between the hand and index fingertip during $3 D$ rotations. The relative orientation between the hand and index finger is constant during rotations.

\subsubsection{Experiment 4-Influence of ferromagnetic object distur- bances}

When the pair of scissors move approximately along the x-axis of the hand frame, the largest estimated distance error was 10.6\%, as shown in Fig 6.13a. When the pair of scissors moved approximately along the y-axis, the largest distance error is $32.3 \%$, as shown in Fig 6.13b. The distance error is related to the direction of the pair of scissors.

\subsection{Discussion}

We proposed and evaluated a magnetometer-magnet based setup for the estimations of interested fingertip position relative to the hand, with the assumption that the orientation of the fingertip relative to the hand is known. The precision of the proposed method is comparable to the precision reported in the literature [26, 102]. To our best knowledge, three studies performed kinematic measurements in human subjects and compared their results with the OTS system. They exploited a similar IMMU-based system with one IMMU attached to each segment of the fingers and a kinematic model of 


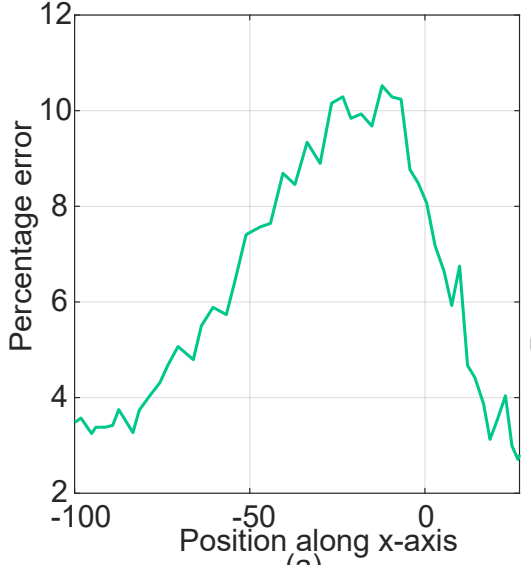

(a)

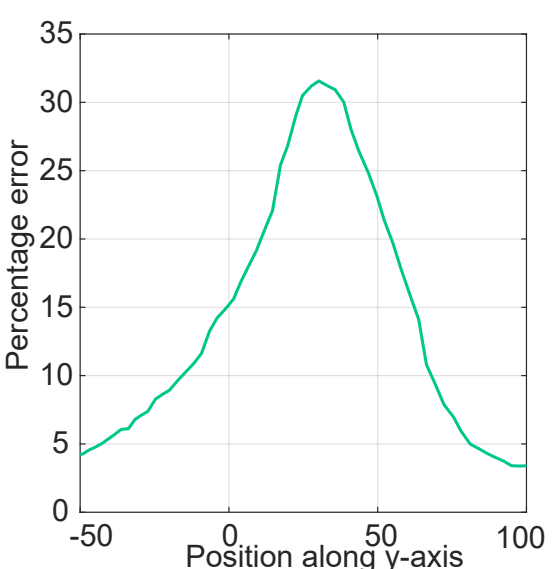

(b)

Figure 6.13: Relative position errors between the hand and the index finger in the presence of ferromagnetic disturbances. (a) and (b) show the distance error with trajectories of the ferromagnetic object along $x$-axis and $y$-axis. The moving direction can be seen in Fig 6.9h.

Table 6.1: Distance errors of the estimated relative position with the different methods during different movements

\begin{tabular}{cccc}
\hline Items & $\begin{array}{c}\text { Kortier and } \\
\text { Noord et al. }\end{array}$ & $\begin{array}{c}\text { Salchow-Hömmen } \\
\text { et al. }\end{array}$ & $\begin{array}{c}\text { The proposed } \\
\text { method }\end{array}$ \\
\hline iflexext $(\mathrm{cm})$ & 0.5 & 0.5 & 0.6 \\
Pinch $(\mathrm{cm})$ & $1.4 \sim 2.1$ & 1.5 & 0.8 \\
\hline
\end{tabular}

the hand. Compared with Kortier et al.'s and Noort et al.'s work [12, 102], Salchow-Hömmen et al. used biomechanical constraints to reduce the influences of magnetic disturbances [26]. The comparison of distance errors during the flexion and extension of the index finger and pinch movement are shown in Table III. Combining our method with a kinematic model could potentially further reduce the relative position estimation error if the model is sufficiently accurate, which is not self-evident. The advantage of not using a kinematic model is that it does not require measurement or estimation of the orientation of the intermediate finger segments between the hand and finger tips.

Compared with the OTS, the estimated position error of fingertip rela- 
tive to the hand was around $10 \mathrm{~mm}$. It is important to discuss the potential sources of remaining estimation errors. The first source is the geomagnetic field disturbance. Although the geomagnetic field was at least 3 times smaller than the magnetic field generated by the magnet, it still played an important role for the estimation errors, as indicated in Fig 6.12. In order to investigate the size of influences, we used the orientation of fingertips from the OTS in its own coordinate frame to obtain the geomagnetic field, then transferred it to the sensor frame and subtracted it from magnetometer outputs. The median errors reduced from $8.0 \%$ and $4.9 \%$ to $6.2 \%$ and $4.0 \%$ during the flexion and extension of the index finger and thumb. The second error source is the direction of the magnet. The best direction of the magnet that caused least error for the index finger was $\alpha=0^{\circ}$ while the worst option was $\alpha=90^{\circ}$. $\alpha$ was set as $\alpha=45^{\circ}$ when we considered both the index finger and thumb, which caused unwanted errors for both fingers. Thirdly, we used the positions of markers from the OTS as the relative position reference. However, the centers of the markers did not coincide with the center of the magnet and the center of the magnetometer. Although we roughly estimated the relative position between them, the inaccuracy of this estimation caused errors when the estimation of relative position was compared with the OTS reference. Fourthly, the approximation of the magnet as a dipole model caused errors for the relative position estimation. We estimate that the fifth error source is the noise from the magnetometer, which can not be compensated for by the calibration procedures. The influences of the fourth and the fifth error sources are much smaller compared with previous error sources.

In order to localize a magnetic dipole, at least two TAMs are required without considering the common geomagnetic field, while at least three TAMs are required when including the geomagnetic field. In the latter case, the third TAM is used to measure and subtract the common geomagnetic field [42, 169]. In our research, only one magnetometer is exploited because of the limited space on the fingertip. Therefore, two assumptions are made: 1) the relative orientation is known. 2) the magnetic field induced by the magnet is much stronger than the geomagnetic field. The accuracy of the estimated fingertip positions relative to the hand is closely related to the assumptions. One possible solution to get rid of two assumptions is to add more TAMs. Certain intervals are needed between TAMs in order to measure differential magnetic field with adequate accuracy. If we have two TAMs on the fingertips of interest, the relative orientation is not required. If 
we have more than three TAMs, neither of the assumptions is required.

When adding new TAMs is not achievable, such as the configuration in our approach, the gyroscope and accelerometer combined with TAM can be used to reduce the impact of the geomagnetic field. As shown in Fig 6.14, we obtain the inclination $\boldsymbol{C}_{g}^{s i}$ of the fingertip with the gyroscope and accelerometer, which is compensated by the gravity sensed by the accelerometer and thus does not have drift issues. The fused inclination $\boldsymbol{C}_{g}^{s i}$ can only be used to compensate the $\mathrm{z}$-component of the geomagnetic field in the sensor frame. In this way, the z-component of the geomagnetic field disturbance is reduced. The performance of this method depends on the location on earth since the z-component of the geomagnetic field varies at different locations.

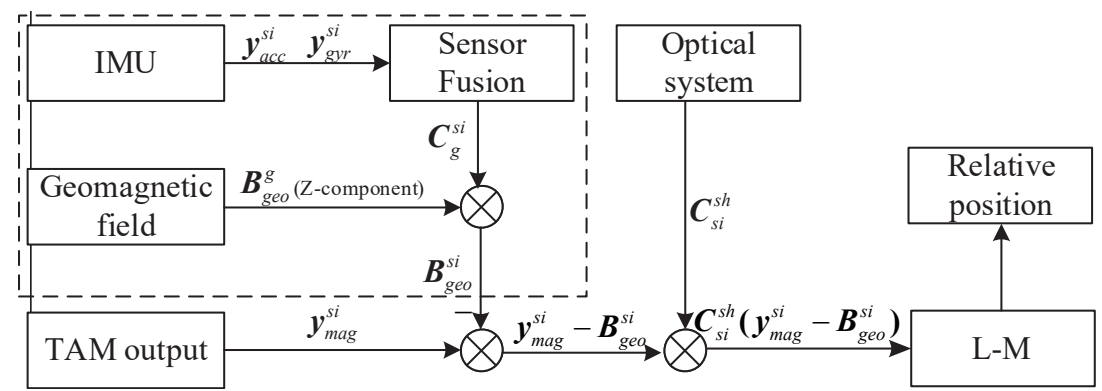

Figure 6.14: Illustration of the position improvement by compensating the z-axis component of geomagnetic field. $\boldsymbol{B}_{\text {geo }}^{g}$ is the z-axis component of geomagnetic field.

Compared with Kortier et al.'s and Salchow Hommen et al's work [102, 26], the volume of our setup is larger, especially the components on the fingertips, which happens for two reasons: firstly, in order to make the system to be easy to wear, we made a 3D printed coat for the components on fingertips and dorsal side of the hand. However, it made the system larger. Secondly, our system reserved an interface and related peripheral circuits for the pressure sensor on fingertips. Since we may integrate pressure information of fingertip in the future, which also enlarge the volume of the system. In this work, we were concentrating on the algorithm for the fingertip position estimation relative to the hand and paid less attention to the shape design. In the future, the design of the entire device can be optimized. Firstly, the system can be smaller. Systems in our work and related research [102, 26] exploited same component MPU9250 $(3 \mathrm{~mm} * 3 \mathrm{~mm} * 1 \mathrm{~mm})$ on the fingertip, which means the system in this work can be as smaller as others, or even 
smaller after optimization. Secondly, all the connections inside the system were wired, which were not convenient to users and can be replaced with wireless connections. The sensor data can be transferred by the Bluetooth etc.

As the strength of the magnet is much stronger than the geomagnetic field, it may cause unwanted forces if it is used near iron objects, such as iron tables or chairs. For a next step, the method to estimate relative positions using a permanent magnet and TAM, assuming known relative orientations, is to be combined with an estimate of relative orientation, as presented in our previous paper [161], in which the relative orientation was estimated based on IMUs only.

\subsection{Conclusion}

A new method was proposed to estimate relative positions between the hand and fingertips of interest with known relative orientations: a magnet on the dorsal side of the hand and an IMMU on the fingertip. The objective of this approach was to avoid using prior information on the length of each segment and reduce the number of IMMUs. The relative position was estimated with the L-M method. During joint movements, the norm of the position error was $4.9 \%, 8.0 \%, 8.7 \%$ and $6.4 \%$ during index finger flexion, thumb flexion, thumb abdution and pinch movement respectively. During functional tasks, the distance error was $8.2 \% \sim 9.4 \%$ when the participant grasped a plastic cube from one position to another position. Influences of the geomagnetic disturbance and the ferromagnetic disturbance were investigated. In conclusion, the proposed method is a promising approach for the hand motion tracking with comparable performance as previous methods that applied a kinematic model of the fingers and additional IMMU's on intermediate finger segments.

\section{Appendix C}

\section{C.1 Proof of existence of position estimation}

When the orientation $\boldsymbol{C}_{m}^{s}$ is unknown, the strength of the measured magnetic 
field can be exploited. From Eq (6.6), we find:

$$
\left|\hat{\boldsymbol{y}}_{m a g}^{s}\right|=\sqrt{\left(\boldsymbol{B}_{m}^{m}\right)^{T}\left(\boldsymbol{B}_{m}^{m}\right)}=\frac{\mu_{0} M_{m}}{4 \pi r_{m}^{3}} \sqrt{1+3 \cos ^{2} \theta}
$$

where $\theta$ is the angle between the position $\boldsymbol{r}_{m}$ and the magnetic moment $\boldsymbol{M}_{m}$. $\hat{\boldsymbol{y}}_{\text {mag }}^{\text {sag }}$ is

$$
\hat{\boldsymbol{y}}_{m a g}^{s}=\boldsymbol{A}^{-1} \boldsymbol{y}_{m a g}^{s}-\boldsymbol{b}
$$

There are infinite solutions for position $\boldsymbol{r}_{m}$ with magnetometer output $\hat{\boldsymbol{y}}_{\text {mag }}^{s}$ based on Eq (24), which cannot be uniquely determined. However, the relation between distance $r_{m}$ and the angle $\theta$ is known. Eq (24) represents a surface in 3D space.

When the orientation $\boldsymbol{C}_{m}^{S}$ is known, the possible solutions can be divided into two cases: whether the magnetic moment $\boldsymbol{M}_{m}$ is perpendicular to the position $\boldsymbol{r}_{m}$.

Case I : $\boldsymbol{M}_{m}$ is perpendicular to $\boldsymbol{r}_{m}$.

In this case, $\theta=\pi / 2$, Eq (6.2) can be simplified as:

$$
\boldsymbol{B}_{m}^{m}=-\frac{\mu_{0}}{4 \pi} \frac{M_{m} \boldsymbol{e}_{z}}{r_{m}^{3}}=-\frac{\mu_{0}}{4 \pi} \frac{\boldsymbol{M}_{m}}{r_{m}^{3}}
$$

Based on Eq (6.11), distance $r_{m}$ can be estimated with $\boldsymbol{B}_{m}^{m}$, not the position $\boldsymbol{r}_{m}$. Since positions with the same distance $r_{m}$ share the same magnetic field $\boldsymbol{B}_{m}^{m}$, the possible positions $r_{m}$ degenerate from a surface to a circle in 3D space with the condition $\boldsymbol{M}_{m} \perp \boldsymbol{r}_{m}$, as shown in Fig $5 \mathrm{~b}$.

Case II : $\boldsymbol{M}_{m}$ is not perpendicular to $\boldsymbol{r}_{m}$.

In this case, Eq (6.2) can be seen as a nonlinear equation set with three equations and three unknown parameters $\boldsymbol{r}_{m}$. Two possible solutions $\pm \boldsymbol{r}_{m}$ can be obtained with $\boldsymbol{B}_{m}^{m}$, as shown in Fig 6.3c. The proof details are as below.

As the magnetic field measured by the TAM is from the magnet, we know the position of the magnet must exist. The proof equals to: given two magnetic fields $\boldsymbol{B}_{m 1}$ and $\boldsymbol{B}_{m 2}$ that satisfy $\boldsymbol{B}_{m 1}=\boldsymbol{B}_{m 2}$, the possibly corresponding positions $\boldsymbol{r}_{m 1}$ and $\boldsymbol{r}_{m 2}$ have the relation

$$
\boldsymbol{r}_{m 1}= \pm \boldsymbol{r}_{m 2}
$$


Based on Eq (6.15) and the assumption $\boldsymbol{B}_{m 1}=\boldsymbol{B}_{m 2}$, we obtain

$$
\begin{aligned}
\boldsymbol{B}_{m 2} \times \boldsymbol{r}_{m 2} \cdot \boldsymbol{B}_{m 1} & =-\frac{3\left(\boldsymbol{r}_{m 1}^{T} \boldsymbol{M}_{m}\right) \boldsymbol{M}_{m} \times \boldsymbol{n}_{\boldsymbol{r}_{m 2}} \cdot \boldsymbol{n}_{\boldsymbol{r}_{m 1}}}{r_{m 1}^{4} r_{m 2}^{2}} \\
& =-\frac{3\left(\boldsymbol{r}_{m 1}^{T} \boldsymbol{M}_{m}\right)\left(\boldsymbol{n}_{\boldsymbol{r}_{m 2}} \times \boldsymbol{n}_{\boldsymbol{r}_{m 1}}\right) \cdot \boldsymbol{M}_{m}}{r_{m 1}^{4} r_{m 2}^{2}}=\boldsymbol{0}
\end{aligned}
$$

From the assumption, we know $\boldsymbol{r}_{m 1}^{T} \boldsymbol{M}_{m} \neq 0$. Then we obtain following equation Based on Eq (28).

$$
\left(\boldsymbol{n}_{\boldsymbol{r}_{m 2}} \times \boldsymbol{n}_{\boldsymbol{r}_{m 1}}\right) \cdot \boldsymbol{M}_{m}=\mathbf{0}
$$

Then, $\boldsymbol{M}_{m}$ can be expressed as

$$
\boldsymbol{M}_{m}=k_{1} \boldsymbol{r}_{m 1}+k_{2} \boldsymbol{r}_{m 2}
$$

since $\boldsymbol{M}_{m} \neq \boldsymbol{0}, k_{1}$ and $k_{2}$ are not zero simultaneously. Combining with Eq (6.15), we obtain

$$
\begin{gathered}
\boldsymbol{B}_{m 1}=\frac{\mu_{0}}{4 \pi}\left(\left(\frac{2 k_{1} r_{m 1}^{2}+3 k_{2} \boldsymbol{r}_{m 1}^{T} \boldsymbol{r}_{m 2}}{r_{m 1}^{5}}\right) \boldsymbol{r}_{m 1}-\frac{k_{2}}{r_{m 1}^{3}} \boldsymbol{r}_{m 2}\right) \\
\boldsymbol{B}_{m 2}=\frac{\mu_{0}}{4 \pi}\left(-\frac{k_{1}}{r_{m 2}^{3}} \boldsymbol{r}_{m 1}+\left(\frac{2 k_{2} r_{m 2}^{2}+3 k_{1} \boldsymbol{r}_{m 2}^{T} \boldsymbol{r}_{m 1}}{r_{m 2}^{5}}\right) \boldsymbol{r}_{m 2}\right)
\end{gathered}
$$

since $\boldsymbol{B}_{m 1}=\boldsymbol{B}_{m 2}$, the following equation is obtained based on Eq (31) and (32)

$$
\left(a_{11} k_{1}+a_{12} k_{2}\right) \boldsymbol{r}_{m 1}-\left(b_{11} k_{1}+b_{12} k_{2}\right) \boldsymbol{r}_{m 2}=0
$$

where $a_{11}, a_{12}, b_{11}, b_{12}$ can be expressed as

$$
\left\{\begin{array}{l}
a_{11}=\frac{2}{r_{m}^{3}}+\frac{1}{r_{m 2}^{3}}, a_{12}=\frac{3 \boldsymbol{r}_{m 1}^{T} \boldsymbol{r}_{m 2}}{r_{m 2}^{5}} \\
b_{11}=\frac{3 \boldsymbol{r}_{m 1}^{T} \boldsymbol{r}_{m 2}}{r_{m 2}^{5}}, b_{12}=\frac{2}{r_{m 2}^{3}}+\frac{1}{r_{m 1}^{3}}
\end{array}\right.
$$

if $\boldsymbol{n}_{r 1} \neq \pm \boldsymbol{n}_{r 1}$, we can obtain following equation based on Eq (33)

$$
\left\{\begin{array}{l}
a_{11} k_{1}+a_{12} k_{2}=0 \\
b_{11} k_{1}+b_{12} k_{2}=0
\end{array}\right.
$$

However, Eq (35) only has zero solutions $k_{1}=0, k_{2}=0$ because of following equations 


$$
\begin{gathered}
a_{11} b_{12}=\frac{5}{r_{m 1}^{3} r_{m 2}^{3}}+\frac{2}{r_{m 1}^{6}}+\frac{2}{r_{m 2}^{6}}>\frac{9}{r_{m 1}^{3} r_{m 2}^{3}} \\
b_{11} a_{12}=\frac{9 \cos ^{2} \theta}{r_{m 1}^{3} r_{m 2}^{3}}<\frac{9}{r_{m 1}^{3} r_{m 2}^{3}} \\
a_{11} b_{12}-b_{11} a_{12}>0
\end{gathered}
$$

which is contradict to the assumption that $k_{1}$ and $k_{2}$ are not zero simultaneously. Thus, the assumption $\boldsymbol{n}_{r 1} \neq \pm \boldsymbol{n}_{r 2}$ is not true. On the contrary, we obtain

$$
\boldsymbol{n}_{r 1}= \pm \boldsymbol{n}_{r 2}
$$

Then, we obtain $\cos ^{2} \theta_{1}=\cos ^{2} \theta_{2}$. Combined with Eq (24), we obtain

$$
r_{m 1}=r_{m 2}
$$

Finally, we proof Eq (27) based on Eq (39) and Eq (40).

\section{C.2 Synchronization and alignment}

The synchronization and alignment are done as follows:

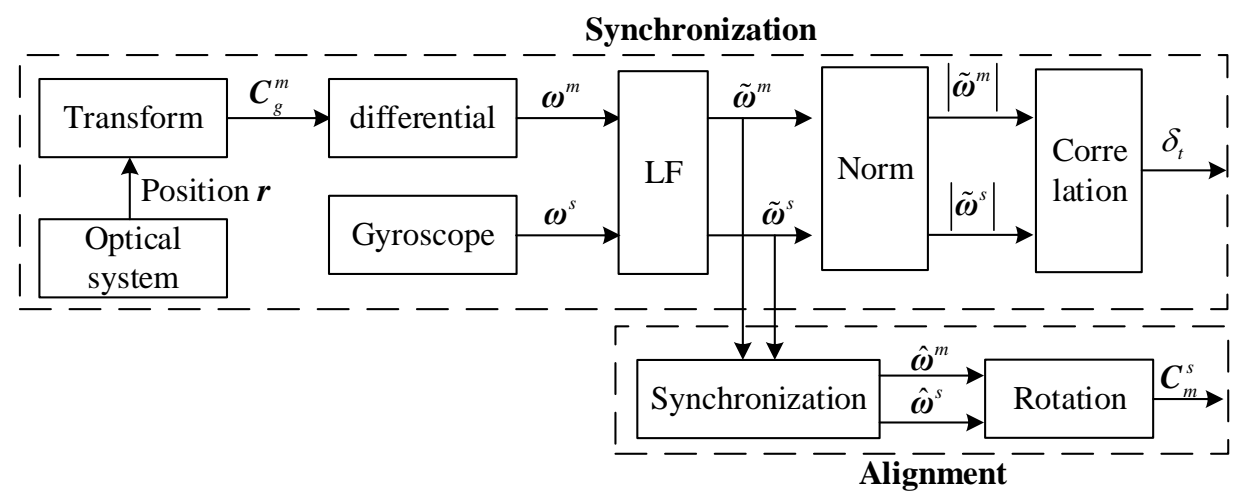

Figure 15: Illustration of the synchronization and alignment.The angular velocity from the OTS contained high frequency noise, low-pass filter $(L F)$ was used to reduce the noise. The OTS and gyroscope use the same LF to reduce the time delay of LF. The passband frequency and stopband frequency were set as $8 \mathrm{~Hz}$ and $15 \mathrm{~Hz}$ respectively. Alignment was done after the synchronization. 


\section{Hand-finger pose estimation using few inertial and magnetic sensors and a magnet}

This chapter is accepted as a journal article: Yang, Z., Yan, S., van Beijnum, B. J. F., Li, B., \& Veltink, P. H. (2021). Hand-finger pose estimation using inertial sensors, magnetic sensors and a magnet. IEEE Sensors Journal. 


\title{
Hand-finger pose estimation using inertial sensors, magnetic sensors and a magnet
}

\begin{abstract}
Hand-finger motion tracking based on inertial and magnetic measurement units (IMMUs) has become a well-accepted method in many applications such as rehabilitation and virtual reality. However, existing systems require an IMMU on each segment of the whole hand and known length of each segment, which is hard to achieve for users. Therefore, we proposed a small sensor configuration with three IMMUs and a magnet to estimate poses of interested fingertips relative to the hand. The relative orientations were estimated by integrating relative angular velocity and fused with relative orientation estimation during time intervals when the whole hand moved or rotated as one object. The relative positions were estimated by using a magnetic dipole model of the magnet and prior estimated relative orientations. The performance has been demonstrated in multiple experiments. For whole hand rotation and functional grasping or writing experiments, the estimated errors of index fingertip position and orientation relative to the hand were $8.0 \sim 9.8 \mathrm{~mm}$ and $5.7^{\circ} \sim 11.2^{\circ}$. For index finger flexion and pinching, the estimated errors of index fingertip position and orientation relative to the hand were $16.2 \sim 25.2 \mathrm{~mm}$ and $20.6^{\circ} \sim 21.7^{\circ}$.
\end{abstract}




\subsection{Introduction}

Hand-finger motion tracking is a challenging problem and of wide interest in various applications, such as hand rehabilitation, enhancement of athletic performance and virtual reality $[170,171,172]$. The problem can be tackled by state-of-the-art systems, such as optical tracking systems (OTSs), electromagnetic tracking systems (EMTSs) or inertial tracking systems.

OTSs such as Vicon motion systems have high position accuracy and are often used as references. Typical drawbacks of OTSs are requirements of lab environment and line-of-sight problems etc $[160,173]$. EMTSs such as Aurora systems have no line-of-sight occlusion. However, their drawbacks are that large volume emitters are required and they are susceptible to external ferromagnetic objects $[174,175]$.

What makes the use of the system based on inertial and magnetic sensors (IMMUs) especially attractive in comparison with the OTS and EMTS is that ambulatory assessments can be performed out of lab environments $[27,28]$. Moreover, it can directly measure acceleration and angular velocity. Based on the raw data, orientation can be obtained by integrating the angular velocity provided with initial orientation. Owing to the drift errors, orientation cannot be estimated accurately for periods longer than dozens of seconds. Fortunately, drift issues can be solved by fusing the data from accelerometers and magnetometers, where accelerometers measure gravity and magnetometers measure geomagnetic field [176, 177, 178]. The performance of the compensation is related to non-gravitational accelerations and ferromagnetic disturbances. Compared with the orientation estimation, the position estimation based on inertial sensors seems to be more difficult, because extracted accelerations usually contain relatively larger errors and strapdown navigation, including double integration of the extracted accelerations makes the position estimation drift quite rapidly [179]. An alternative method for position estimation based on IMMUs is applying forward kinematics when the orientation and length of each segment are known. Several systems were designed to assess hand kinematics by using forward kinematics based on IMMUs, which were placed on each segment of the whole finger [102, 180, 181]. Subsequently, Salchow-Hommen et al. improved the robustness to magnetic disturbances by reducing magnetometers and simplifying 3DOF joint movements to 2DOF joint movements [26].

However, previous IMMU-based systems have several major drawbacks. Firstly, an IMMU on each finger segment is required, which are difficult 
to wear and calibrate since each IMMU needs to be well fixed and aligned with corresponding segment. Secondly, too much irrelevant information is estimated that increases the complexity of calculations. In many cases, we are only interested in the relative poses between fingertips and the dorsal side of the hand, but we need to estimate poses of all segments in order to use forward kinematics. Furthermore, errors of estimated positions accumulate along articulated chains because of the uncertainty of segment lengths and error parameters estimated by sensor to segment calibrations.

Compared with forward kinematics, a more robust solution to improve the position performance of IMMU-based systems is to fuse external position systems such as OTSs, ultrasound systems or EMTSs [182, 183, 184]. Weenk et al. proposed a method to estimate relative foot positions with inertial and ultrasonic sensors [119]. The position accuracy was improved compared with an IMMU-based system since ultrasound sensors provide distance information without drift. Roetenberg et al. proposed a method for ambulatory pose estimation by using IMMUs and orthogonal coils worn on the body [40]. Subsequently, Schepers et al. improved the hardware and algorithm [185]. Compared to the fusion of ultrasound systems, EMTSs provide position information instead of distance information, which is more suitable for the compensation of position drift. However, drawbacks still exist. Firstly, the body-worn EMTS is bulky, which hinder the subject's movements. Secondly, it consumes lots of energy, especially when the EMTS needs to cover a large tracking volume. To overcome those drawbacks, one potential method is to use a magnet as a passive emitter instead of active magnetic coils. Whereas, the magnet can only generate magnetic field along one axis instead of three axes like orthogonal coils. Furthermore, the magnetic field generated by the magnet is mixed with geomagnetic field, which cannot be easily extracted. Thus, the system requires more magnetometers as receivers to estimate poses of the magnet. Kortier et al. proposed a system including one magnet on the hand and four magnetometers on the trunk to estimate the pose of the hand relative to the trunk [42].

Inspired by Kortier et al.'s work, this paper presents a further step towards estimation of interested hand-finger poses using a small IMMU-based system and a magnet. The system contains three IMMUs placed on the index fingertip, thumb fingertip and dorsal side of the hand. No IMMU is attached to the intermediate segments. As fingertips are much smaller compared with the trunk, we proposed to only attach one magnetometer per fingertip instead of four. A new method with IMMUs and one magnetometer is proposed to 
estimate finger poses relative to the hand with IMMUs and one magnetometer, which is divided into two steps. Firstly, the relative orientations between the hand and fingertips are estimated based on inertial sensors [161]. Subsequently, relative orientations are combined with magnetometer outputs to estimate the relative positions between the hand and fingertips. The information that the hand and fingers often share approximately the same angular velocity or acceleration if the whole hand is moved or rotated is exploited to compensate the relative direction drift without magnetometers. The contribution of this paper is proposing a small IMMU-based system to estimate relative poses between the hand and fingertips of interest, without known segment lengths or requirement of forward kinematics.

\subsection{Methods}

In order to estimate the fingertip poses relative to the hand, a small IMMUbased system and corresponding algorithms were designed.

\subsubsection{System design}

A traditional system from previous research is shown in Fig 7.1a. The proposed system is shown in Fig 7.1b. Two IMMUs are fixed on the thumb and index fingertip respectively. One IMMU is fixed on the dorsal side of the hand with one magnet attached above. It looks like our system is larger than the system shown in Fig 7.1a. However, we used the same sensor MPU9250 on finger segments. The reasons why it looks larger are: firstly, our system reserved an interface and peripheral circuits for pressure sensor on fingertip, which we may fuse in the future work. However, it occupies a large space. Secondly, in order to make the system easier to wear, we made a 3D printed coat, which also makes our system looks larger.

Compared with previous systems, our system concentrates on the movements of thumb and index fingertips. Thus, IMMUs attached to the middle and proximal phalanx are not included. As a consequence, forward kinematics cannot be used to estimate the relative positions anymore. The magnet on the dorsal side of the hand is used as a passive magnetic source to estimate finger positions relative to the hand. However, the introduction of the magnet disturbs the geomagnetic field measured by magnetometers. Therefore, the magnetometer cannot be used to compensate the direction drift. Instead of 


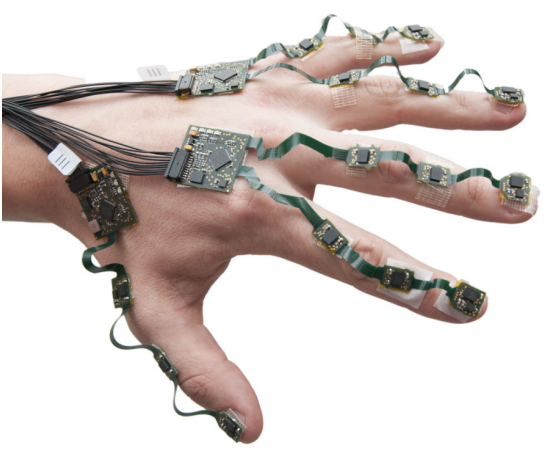

(a)

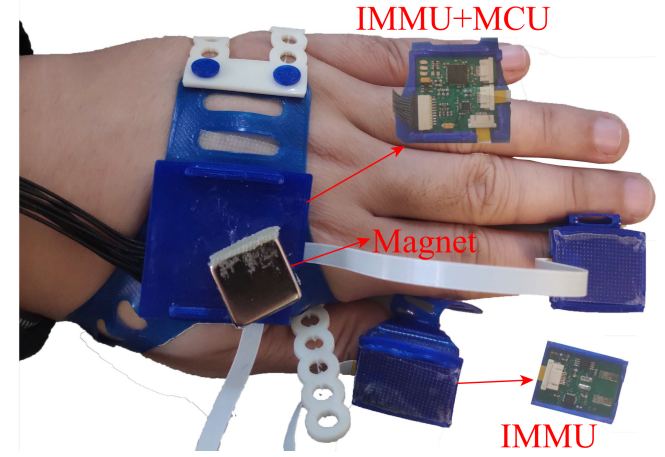

(b)

Figure 7.1: (a) IMMU-based system from Kortier et al.'s work [25], with an IMMU on each segment (b) The proposed small IMMU-based system, one IMMU on the tip of the thumb, one on the index fingertip and one on the dorsal side of the hand, with a magnet attached.

exploiting the geomagnetic field, we exploit the information collected when the hand moves as a whole object to compensate the direction drift.

The process of estimating the $6 \mathrm{D}$ relative poses between the hand and fingertips is shown in Fig 7.2. The relative orientation is estimated first, which is a necessary condition to estimate relative position.

\subsubsection{Estimation of relative orientations with inertial sensors}

As shown in Fig 7.2, the relative orientation between the hand and fingertips is obtained by integrating relative angular velocity, and compensated by the relative inclination and the information collected when the hand moves as a whole object [176].

\section{Estimation of relative orientation change}

The relative angular velocity between the hand and fingertips can be obtained as

$$
\boldsymbol{y}_{g y r}=\boldsymbol{y}_{g y r, h}^{h}-\boldsymbol{C}_{f}^{h} \boldsymbol{y}_{g y r, f}^{f}
$$

where $\boldsymbol{y}_{g y r, h}^{h}$ and $\boldsymbol{y}_{g y r, f}^{f}$ are gyroscope outputs on the hand and fingertips in their own frames. $C_{f}^{h}$ is the relative orientation between the hand and fin- 


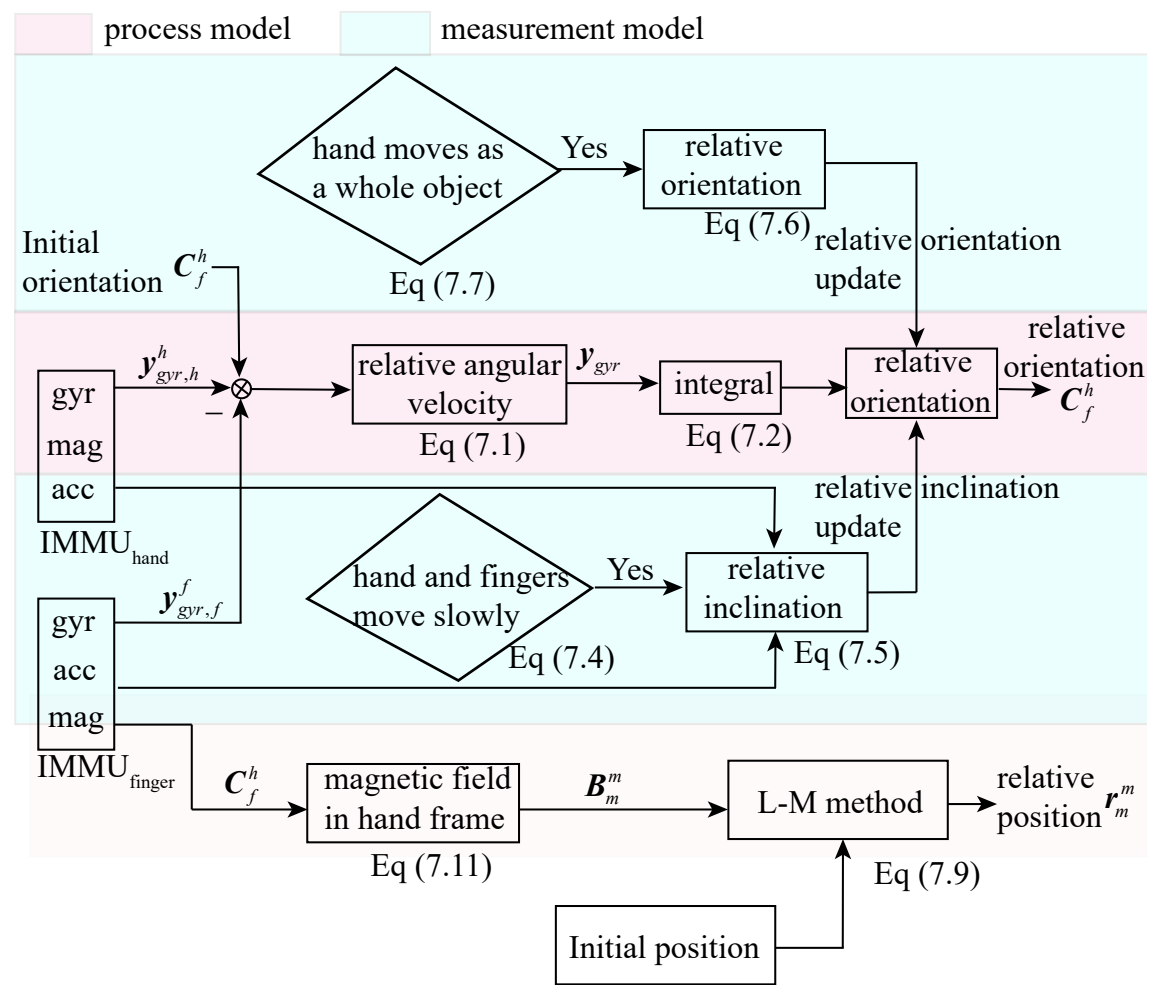

Figure 7.2: Algorithm for the estimating fingertip poses relative to the hand with IMMUs and a magnet.

gertips based on rotation matrix, which can be updated with relative angular velocity $\boldsymbol{y}_{\text {gyr }}$ :

$$
\dot{\boldsymbol{C}}_{f}^{h}=\boldsymbol{C}_{f}^{h}\left[\boldsymbol{y}_{g y r} \times\right]
$$

where $\left[\boldsymbol{y}_{\text {gyr }} \times\right]$ is

$$
\left[\boldsymbol{y}_{\text {gyr }} \times\right]=\left[\begin{array}{ccc}
0 & -y_{\text {gyr }, z} & y_{\text {gyr }, y} \\
y_{\text {gyr }, z} & 0 & -y_{g y r, x} \\
-y_{g y r, y} & y_{g y r, x} & 0
\end{array}\right]
$$

$y_{g y r, x}, y_{g y r, y}, y_{g y r, z}$ are three components of $\boldsymbol{y}_{g y r}$. The update of orientation $\boldsymbol{C}_{f}^{h}$ based on Eq (7.2) may introduce drift. Therefore, this orientation estimate needs to be fused with some other information about relative orientation that does not drift. 


\section{Estimation of relative inclination using accelerometers}

When the hand and fingers move or rotate slowly, the non-gravitational acceleration is negligible compared with the gravity.

$$
||\left|\boldsymbol{y}_{a c c, h}^{h}\left\|_{2}-g\left|<\varepsilon_{a},\right||| \boldsymbol{y}_{a c c, f}^{f}\right\|_{2}-g\right|<\varepsilon_{a}
$$

where $\boldsymbol{y}_{a c c, h}^{h}$ and $\boldsymbol{y}_{a c c, f}^{f}$ are accelerometer outputs in their own frames. $g$ is the local gravity. $\varepsilon_{a}$ guarantees the non-gravitational acceleration is not too large when the relative inclination is estimated. The relation of accelerometers between the hand and fingers can be approximated as

$$
\boldsymbol{y}_{a c c, h}^{h} \approx \boldsymbol{C}_{f}^{h} \boldsymbol{y}_{a c c, f}^{f}
$$
Relative orientation estimation under the condition that the hand and
fingers accelerate or rotate approximately as one object

Eq (7.3) can not fully compensate the relative orientation drift caused by the time integral of relative angular velocity. Fortunately, the hand and fingers often move as a whole object during daily tasks. Thus, they frequently share approximately the same acceleration and angular velocity.

$$
\left\{\begin{array}{l}
\boldsymbol{y}_{g y r, h}^{h} \approx \boldsymbol{C}_{f}^{h} \boldsymbol{y}_{\text {gyr,f }}^{f} \\
\boldsymbol{y}_{a c c, h}^{h} \approx \boldsymbol{C}_{f}^{h} \boldsymbol{y}_{a c c, f}^{f}
\end{array}\right.
$$

In order to determine when the hand and fingers moves as a whole object, a decision algorithm was designed

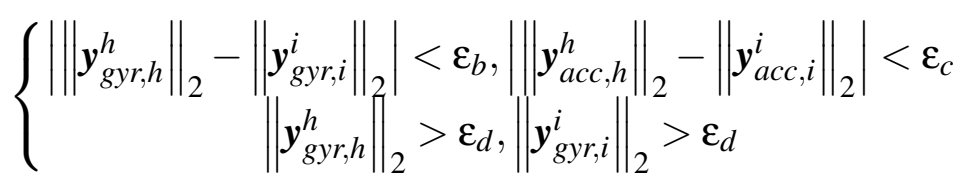

where $\varepsilon_{b}$ and $\varepsilon_{c}$ guarantee the hand and fingers share approximately the same acceleration and angular velocity. $\varepsilon_{d}$ guarantees the hand is rotating rather than stationary. In this way, $\boldsymbol{C}_{f}^{h}$ can be estimated based on Eq (7.4). $\varepsilon_{a, b, c, d}$ are chosen according to the desired movement complexity [161]. In this experiment, the tuning parameters $\varepsilon_{a, b, c, d}$ were set as:

$$
\left\{\begin{array}{c}
\varepsilon_{a}=0.05 g, \varepsilon_{b}=0.1\left\|\boldsymbol{y}_{\text {gyr }, h}^{h}\right\|_{2} \\
\varepsilon_{c}=0.1\left\|\boldsymbol{y}_{a c c, i}^{i}\right\|_{2}, \varepsilon_{d}=0.4 \mathrm{rad} / \mathrm{s}
\end{array}\right.
$$


7.2.3 Estimation of relative positions with known orientations and a magnetometer

\subsubsection{Estimation of relative positions with known orientations and a magnetometer}

When a ferromagnetic object is far from the magnetometer, more than 2.5 times of its length, the object can be approximated as a dipole $[43,186]$. The magnetic field generated by a dipole in its own frame is

$$
\boldsymbol{B}_{m}^{m}=f\left(\boldsymbol{r}_{m}^{m}\right)=\frac{\mu_{0}}{4 \pi}\left(\frac{3\left(\boldsymbol{r}_{m}^{m}\right)^{T} \boldsymbol{M}_{m}^{m} \boldsymbol{r}_{m}^{m}}{\left(r_{m}^{m}\right)^{5}}-\frac{\boldsymbol{M}_{m}^{m}}{\left(r_{m}^{m}\right)^{3}}\right)
$$

where $\mu_{0}$ is the permeability of free space, $\boldsymbol{M}_{m}^{m}$ is the magnetic moment in magnet frame, $\boldsymbol{r}_{m}^{m}$ is the position in magnet frame and $r_{m}^{m}$ is the corresponding distance. $\left(\boldsymbol{r}_{m}^{m}\right)^{T}$ is the transpose of $\boldsymbol{r}_{m}^{m}$. The magnetometer output on the fingertip is

$$
\boldsymbol{y}_{\text {mag }}^{s}=\boldsymbol{C}_{f}^{s}\left(\boldsymbol{C}_{f}^{h}\right)^{-1} \boldsymbol{C}_{m}^{h} \boldsymbol{B}_{m}^{m}+\boldsymbol{B}_{e}^{s}+\boldsymbol{n}_{B}
$$

where $\boldsymbol{B}_{e}^{s}$ is the geomagnetic field in magnetometer frame. $\boldsymbol{n}_{B}$ is the measurement error. $\boldsymbol{C}_{m}^{h}$ is the rotation matrix between the magnet and hand. $\boldsymbol{C}_{f}^{s}$ is the rotation matrix between the magnetometer and fingertips. $\boldsymbol{C}_{m}^{h}$ and $\boldsymbol{C}_{f}^{s}$ can be obtained prior to measurements through calibration procedures. $\boldsymbol{C}_{f}^{h}$ can be obtained as described previously in subsection B on page 2. When the sum of $\boldsymbol{B}_{e}^{s}$ and $\boldsymbol{n}_{B}$ are much smaller than $\boldsymbol{B}_{m}^{m}$ (depending on the required position accuracy), Eq (7.10) can be simplified as

$$
\boldsymbol{B}_{m}^{m} \approx\left(\boldsymbol{C}_{m}^{h}\right)^{-1}\left(\boldsymbol{C}_{f}^{h}\right)\left(\boldsymbol{C}_{f}^{s}\right)^{-1} \boldsymbol{y}_{m a g}^{s}
$$

$\boldsymbol{r}_{m}^{m}$ can be estimated with Levenberg-Marquardt (L-M) method based on Eq (7.11). However, two possible solutions will be estimated since

$$
f\left(\boldsymbol{r}_{m}^{m}\right)=f\left(-\boldsymbol{r}_{m}^{m}\right)
$$

The ghost solution can be eliminated by a good initial value or the general range of the finger movement.

\subsection{Experimental methods}

\subsubsection{Experiment setups}

MPU9250 (InvenSense) was chosen as the IMMU. The magnetometer and accelerometer were sampled at $100 \mathrm{~Hz}$. The gyroscope was sampled at 200 
Hz. All the data was transmitted to a PC through a USB connection. A 1.1 $\mathrm{cm} \times 1.1 \mathrm{~cm} \times 1.1 \mathrm{~cm}$ magnet with the magnetic moment of $3.2 \mathrm{~A} \cdot \mathrm{m}^{2}$ was chosen as a passive magnetic source based on two criteria: (1) the magnetic field generated by the magnet should be much stronger than the geomagnetic field in the movement range. (2) The length of the magnet should be much smaller than the estimated distance. The Vicon system with 8 cameras was used to provide the position and orientation reference. Two clusters of markers were attached to the fingertips of thumb and index finger. A cluster of markers was attached to the surface of the magnet. Each cluster contained three markers. The sample frequency of Vicon system was $100 \mathrm{~Hz}$.

\subsubsection{References from the Vicon system}

The position reference was obtained from the measurement of one marker. The orientation reference was estimated from a cluster of markers. For each cluster, we obtain two vectors $\boldsymbol{r}_{1}, \boldsymbol{r}_{2}$ spanning a configuration of three markers. Then the orientation of the marker frame can be calculated as:

$$
\boldsymbol{C}_{M}^{g}=\left[\begin{array}{lll}
\frac{\boldsymbol{n}_{\boldsymbol{r}_{2}} \times \boldsymbol{n}_{\boldsymbol{r}_{3}}}{\mid \boldsymbol{n}_{\boldsymbol{r}_{2} \times \boldsymbol{n}_{\boldsymbol{r}_{3}} \mid}} & \boldsymbol{n}_{\boldsymbol{r}_{2}} & \boldsymbol{n}_{\boldsymbol{r}_{3}}
\end{array}\right]
$$

where $\boldsymbol{n}_{\boldsymbol{r}_{3}}=\boldsymbol{n}_{\boldsymbol{r}_{1}} \times \boldsymbol{n}_{\boldsymbol{r}_{2}} /\left|\boldsymbol{n}_{\boldsymbol{r}_{1}} \times \boldsymbol{n}_{\boldsymbol{r}_{2}}\right| . \boldsymbol{n}_{\boldsymbol{r}_{1}}$ and $\boldsymbol{n}_{\boldsymbol{r}_{2}}$ are normalized vectors corresponding to $\boldsymbol{r}_{1}$ and $\boldsymbol{r}_{2} . \boldsymbol{C}_{M}^{g}$ is the orientation of optical markers in the global frame of the OTS. The global frame of OTS was determined by the calibration procedures of the OTS. Through the rotation matrix between the marker frame and sensor frame, the orientation assessed by the OTS can be easily transferred to the sensor frame.

\subsubsection{Synchronization and alignment between the IMMU and OTS}

The IMMU and Vicon system were two independent systems. They needed to be synchronized and aligned because the Vicon system provided the orientation and position reference for IMMUs. Angular velocities obtained from the IMMU-based system and Vicon system were used to achieve this goal.

For the IMMU system, angular velocities could be measured by the gyroscope. For the Vicon system, the orientation can be obtained based on the cluster of markers. Subsequently, angular velocities could be estimated. We maximized the correlations of both norms to achieve the synchronization. 
The alignment can be achieved by the following equation

$$
\boldsymbol{C}_{M}^{s}=\operatorname{argmin}\left(\sum_{k=1}^{N}\left\|\left(\hat{\boldsymbol{\omega}}^{s}\right)_{k}-\boldsymbol{C}_{M}^{S}\left(\hat{\boldsymbol{\omega}}^{M}\right)_{k}\right\|_{2}^{2}\right)
$$

where $\hat{\boldsymbol{\omega}}^{M}$ is the angular velocity in the marker frame. $\hat{\boldsymbol{\omega}}^{S}$ is the angular velocity in the gyroscope sensor frame. $C_{M}^{S}$ is the rotation matrix from the marker frame to the sensor frame.

\subsubsection{Protocol}

The performance of the proposed method was verified by different movements from 8 participants. The protocol was approved by ethics committee of Faculty of Electrical Engineering, Mathematics and Computer Science of the Univeristy of Twente.

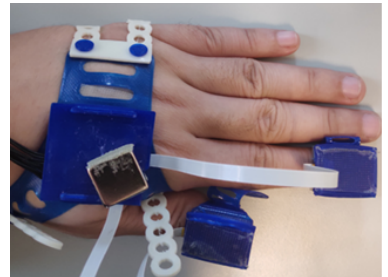

(a)

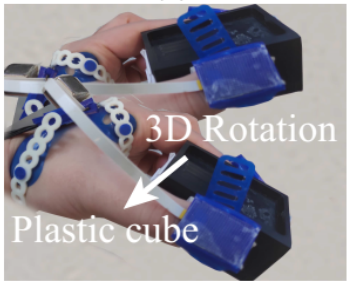

(d)

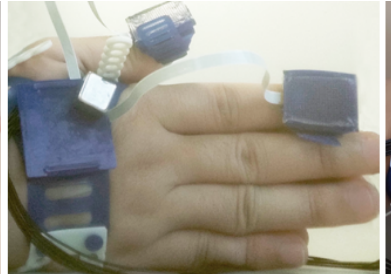

(b)

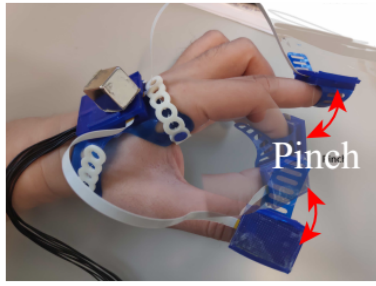

(e)

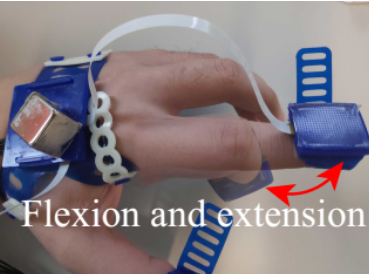

(c)

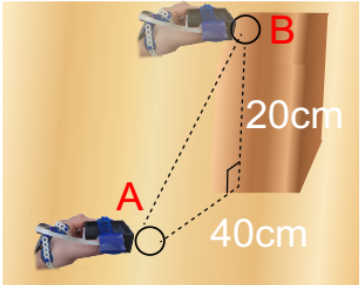

(f)

Figure 7.3: Illustration of the movements in the experiments. (a) Hand flat on the table. (b) Hand vertically on the table. (c) Index finger flexion and extension. (d) Hold a plastic cube and rotate in $3 D$ space, while the relative orientation between the hand and fingers remains unchanged. (e) Pinch movement. (f) Move a plastic cube from point $A$ to point $B$. The horizontal and vertical distance between position $A$ and $B$ are $40 \mathrm{~cm}$ and $20 \mathrm{~cm}$, respectively. 


\section{Sensor to segment calibration}

Before the measurement, IMMUs need to be aligned to segments of the hand. Gyroscopes and accelerometers were exploited to realize such calibration. The procedures are described as follows, more details can be found in related research [102].

(1) Put the hand flat on the table and keep static for $20 \mathrm{~s}$, with the back of the hand facing upward, as shown in Fig 7.3a;

(2) Put two hands together on the table vertically and hold on for $20 \mathrm{~s}$, as shown in Fig 7.3b;

(3) Flex and extend the index finger repeatedly for $20 \mathrm{~s}$, as shown in Fig $7.3 \mathrm{c}$

\section{Initial orientation estimation}

Lay the palm and fingers flat, and then rotate the hand as a whole object along the middle finger for $10 \mathrm{~s}-$ at the rate of 3 times per second.

\section{Experiment 1-3D random rotation without changing relative orienta- tions between the hand and fingertips}

Hold a plastic cube while not changing the relative orientations between the hand and fingers, and then rotate the cube randomly in 3D space, as shown in Fig 7.3d.

\section{Experiment 2-Grasping tasks}

(1) Put the palm flat on the table near initial position A for $10 \mathrm{~s}$;

(2) Grasp the plastic object with tips of the index finger and thumb from the position $\mathrm{A}$ and put it to the position $\mathrm{B}$, and then move the hand to the initial position A, as shown in Fig 7.3f. The horizontal and vertical distances between position $\mathrm{A}$ and $\mathrm{B}$ are $40 \mathrm{~cm}$ and $20 \mathrm{~cm}$ respectively;

\section{Experiment 3-Writing tasks}

Put the palm flat on the table, and then pick up a pen and write for $20 \mathrm{~s}$. 


\section{Experiment 4-Flexion and extension}

Stretch the arm, and then flex and extend the index finger for $20 \mathrm{~s}-$ at the rate of 3 times per second;

\section{Experiment 5-Pinch}

Stretch the arm, and then perform a pinch movement with the thumb and the index finger for $20 \mathrm{~s}$, at the rate of 3 times per second, as shown in Fig 7.3e.

\subsection{Results}

Fig 7.4 shows the 6D pose tracking results of the grasping experiment. It can be seen there is no obvious drift error both for relative orientations and positions. The average errors for estimations of relative positions and orientations are $10.2 \mathrm{~mm}$ and $9.6^{\circ}$ respectively.

Fig 7.5 shows the $6 \mathrm{D}$ pose tracking results of the flexion experiment. It can be seen there are obvious drift errors either for relative orientations or positions. The average errors for estimations of relative positions and orientations are $19.3 \mathrm{~mm}$ and $16.4^{\circ}$ respectively. Compared with the grasping experiment, the flexion experiment did not include the condition that the hand moved as a whole object. Therefore, drift in relative orientation estimation could not be avoid.

Fig 7.6 shows the estimated errors of several movements. Estimated position and orientation errors for the grasping, writing and rotating movements are smaller. During these movements, we had moments when the hand moved as a whole object and obtained enough information to compensate drift errors.

\subsection{Discussion}

We proposed and evaluated a small setup including 3 IMMUs and a magnet to estimate the poses of interested fingertips relative to the hand. Several studies performed movement experiments on human objects and compared results with an optical tracking system. In Kortier et al.'s research where the finger flexion and circular were performed, the estimated position error between the hand and index fingertip was $5.7 \sim 12.4 \mathrm{~mm}$, and the estimated 

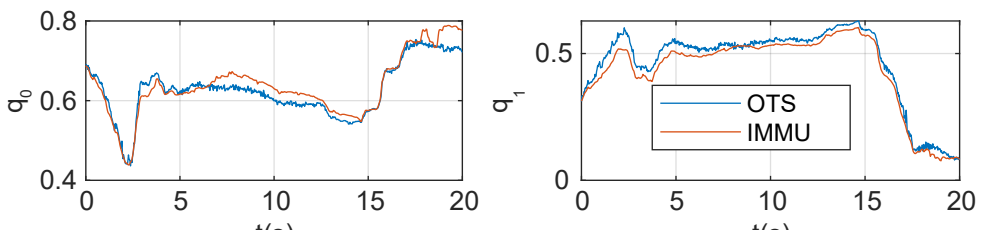

t(s)

t(s)
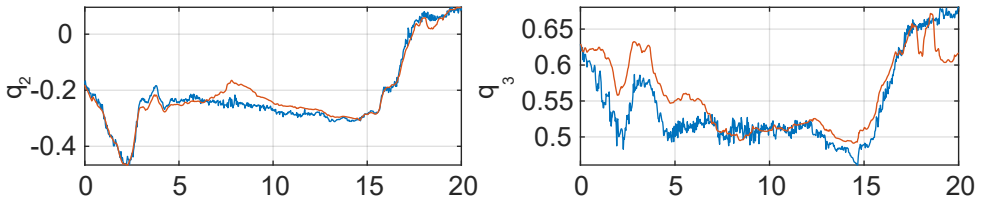

$\mathrm{t}(\mathrm{s})$

$\mathrm{t}(\mathrm{s})$

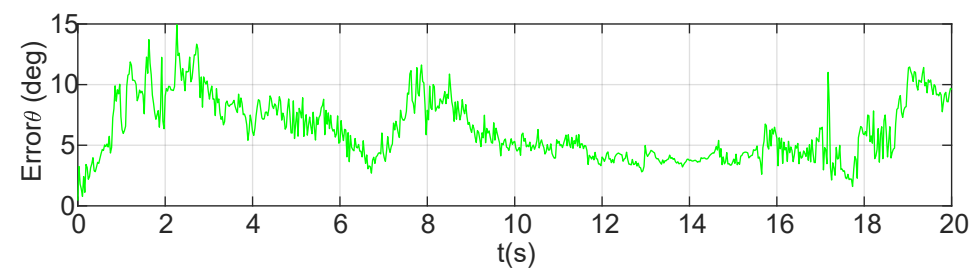

(a)
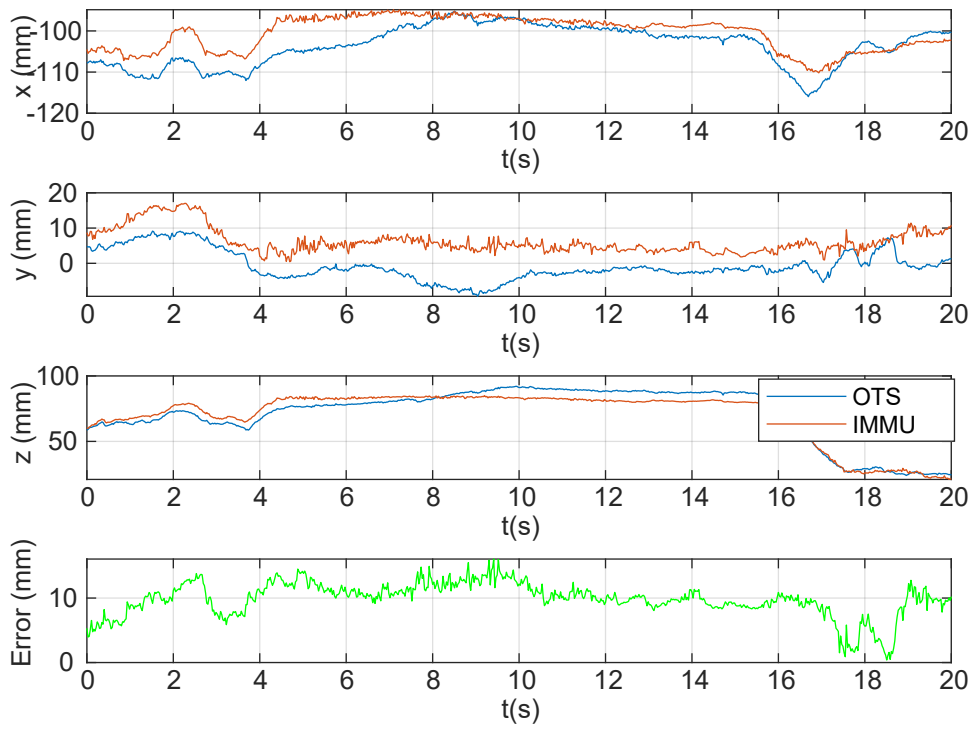

(b)

Figure 7.4: $6 D$ pose tracking results of the grasping experiment. (a) Fingertip orientation relative to the hand in the grasping experiment. (b) Fingertip position relative to the hand in the grasping experiment. 

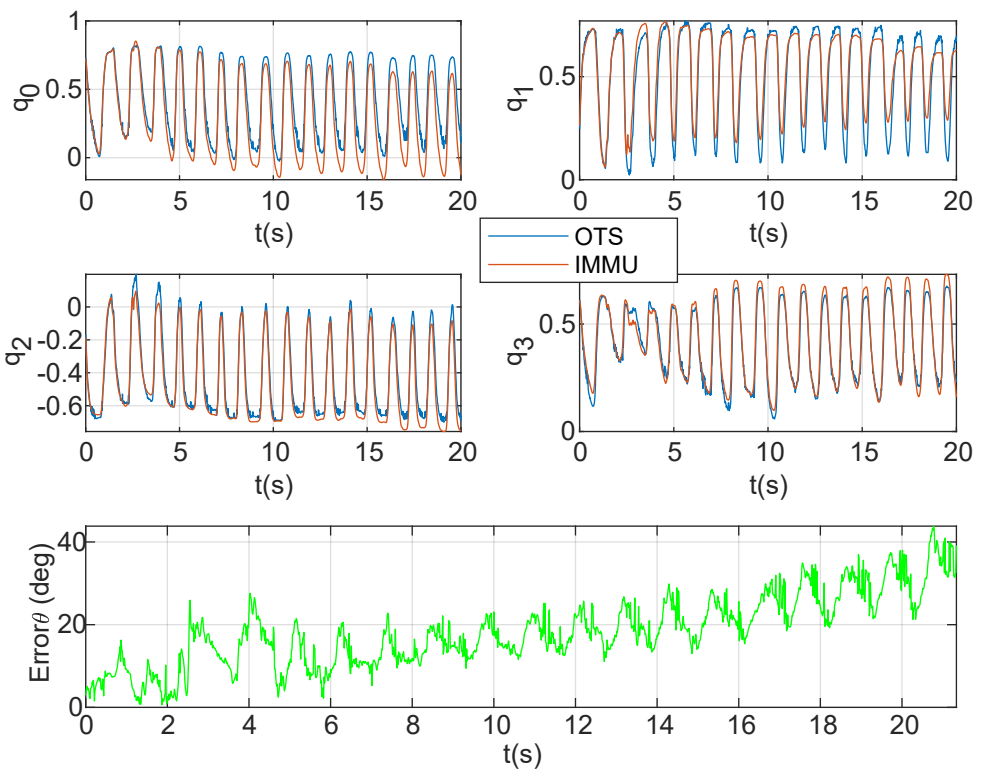

(a)
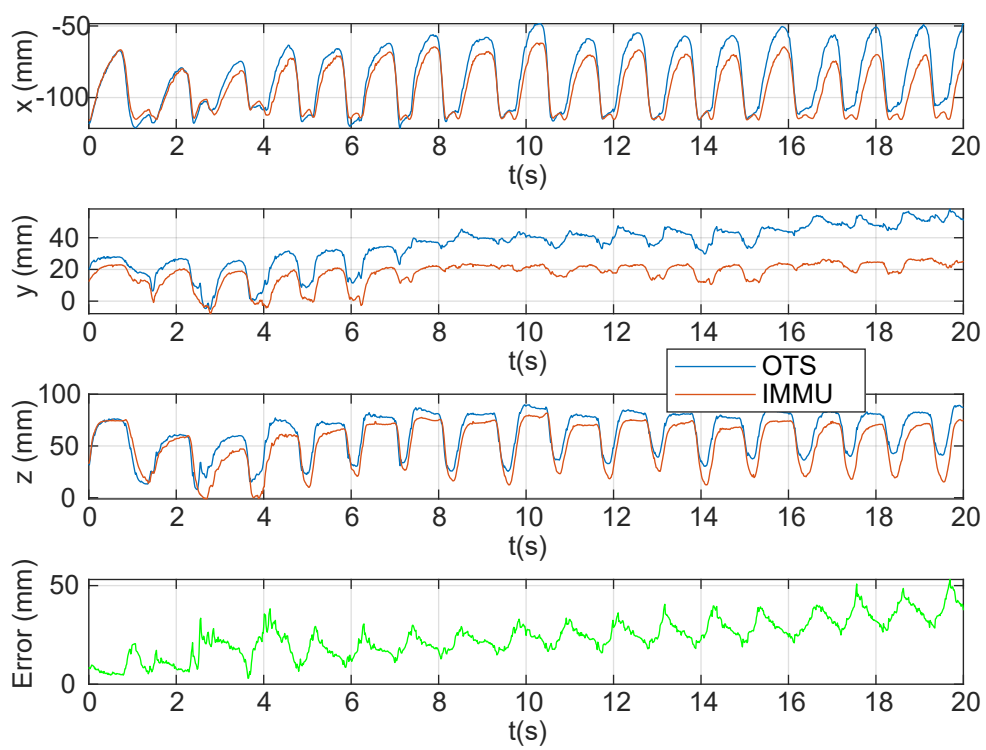

(b)

Figure 7.5: 6D pose tracking results of the flexion experiment. (a) Position tracking in the flexion experiment. (b) Orientation tracking in the flexion experiment. $6 D$ pose tracking results in experiments of index finger flexion and extension. (a) Fingertip orientation relative to the hand orientation in experiments of index finger flexion and extension. (b) Fingertip position relative to the hand position in experiments of index finger flexion and extension. 


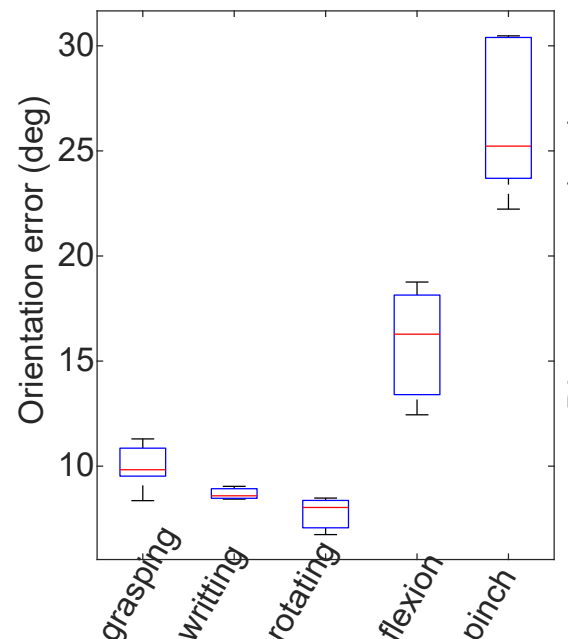

(a)

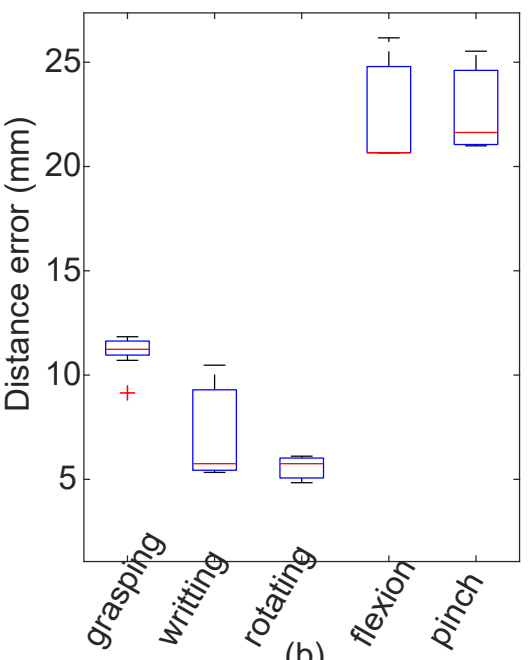

(b)

Figure 7.6: Median pose errors from 8 participants during different movements. (a)Relative Orientation error with different movements. (b) Relative position error with different movements.

Table 7.1: Comparison of the performance of the proposed method with others'

\begin{tabular}{|c|c|c|c|c|}
\hline Items & IMML & Magnet & Position error & Orientation error \\
\hline $\begin{array}{l}\text { Salchow-Hömmen } \\
\text { et al. }{ }^{[1]}\end{array}$ & 16 & No & $5 \sim 21 \mathrm{~mm}$ & $5^{\circ} \sim 16^{\circ}$ \\
\hline Kortier et al. ${ }^{[1]}$ & 18 & No & $5.7 \sim 12.4 \mathrm{~mm}$ & $5.0^{\circ} \sim 8.4^{\circ}$ \\
\hline The proposed method ${ }^{[2}$ & 2] 3 & Yes & $\begin{array}{c}8.0 \sim 9.8 \mathrm{~mm} \\
16.2 \sim 25.2 \mathrm{~mm}\end{array}$ & $\begin{array}{c}5.7^{\circ} \sim 11.2^{\circ} \\
20.6^{\circ} \sim 21.7^{\circ}\end{array}$ \\
\hline
\end{tabular}

1 For Salchow-Hömmen et al.'s and Kortier et al.'s work, the orientation error was refer to the error between two segments, not between the hand and fingertips.

${ }^{2}$ For the proposed method, the performance was related to the quality of the movements. For the first row, there was an opportunity that hand moved as a whole object, which could be used to update the orientation, while there was not such opportunity for the result in the second row.

orientation error was less than $5.0^{\circ} \sim 8.4^{\circ}$ for each segment of the hand during flexion and extension movements. During the repeatability tests, the orientation error for a single posture was less than $2^{\circ}$.

In Salchow-Hömmen et al.'s research where finger flexion and the com- 
bination movement of abduction and flexion were performed, the estimated position error between the hand and index fingertip was $5 \sim 21 \mathrm{~mm}$, and the estimated orientation error was $5 \sim 16$ deg for each segment. Both studies applied an IMMU on each segment of fingers and a kinematic model of the hand.

In comparison, the precision of the proposed method depends on the complexities of movements. For rotating, grasping and writing movements, the orientation error for each segment was not estimated, but the estimated orientation error between the hand and index finger was $5.7^{\circ} \sim 11.2^{\circ}$. The estimated position error between the hand and index finger was $8.0 \sim 9.8 \mathrm{~mm}$. The estimated position and orientation errors are comparable to Kortier et al.'s and Salchow-Hömmen et al.'s results $[102,26]$. For the flexion and pinch movements, the orientation and position errors between the hand and index fingers were $20.6^{\circ} \sim 21.7^{\circ}$ and 16.2 25.2 mm (see Fig 7.6), which are larger than Kortier et al.'s and Salchow-Hömmen et al.'s results [102, 26]. Compared with Kortier et al.'s and Salchow-Hömmen et al.'s systems, the advantages of the proposed method are that IMMUs attached to the intermediate segments and the lengths of finger segments are not required.

For the estimation of relative orientations, the accuracy depends on the complexity of movements. The estimation may have large drift if there is no chance that the whole hand moves or rotates as a whole object. However, the drift can be reduced by applying smoothing techniques, such as Rauch-Tung-Striebel (RTS) smoother, since for the orientation estimation at $t_{k}$, the information during the whole period can be exploited with smoothing technique, while the EKF can only exploit information between $0 \sim t_{k}$.

For the estimation of relative positions, there are several factors that affect the results. Firstly, the accuracy of relative position estimations is related to the relative orientation estimations, since the estimated relative orientations are used as input variables to calculate relative positions. Secondly, the magnetic field generated by the magnet is assumed to be much larger than the geomagnetic field. The estimation errors can be large when the assumption is not true. Thirdly, the model of the magnet is simplified as a dipole model which may cause errors, especially when the distance between the magnetometer and magnet is less than 2.5 times of the magnet's length. Fourthly, disturbances caused by ferromagnetic objects can also affect the accuracy of relative positions. The impact depends on the intensity of the ferromagnetic disturbances. Besides, the optical markers did not coincide with IMMUs. In our experiment, the relative position between them was 
roughly estimated from the experimental photos, thus may contained errors. There are small offset errors in Fig 7.4 and 7.5, which may be caused by the non-coincidence of the marker and IMMU.

The geomagnetic field is assumed as a disturbance and could not be estimated because we only have one magnetometer on the fingertip. If the geomagnetic field can be estimated with more magnetometers, the position accuracy can be improved. Moreover, if we have a magnetometer array with four magnetometers, the relative orientations between the magnet and fingertips can be estimated by using magnetometers alone. In that case, the update of relative orientations does not depend on the complexity of the movements anymore. Then we can obtain good estimations during a long period no matter what type of the movement is. When adding more magnetometers is feasible, the size and strength of magnet can be reduced. Nevertheless, the distance between magnetometers should be large enough that the differential magnetometer field measured by the magnetometer array is much larger than the noise. When adding new magnetometers is not achievable, such as the configuration in our approach, the gyroscope and accelerometer combined with magnetometer can be used to reduce the impact of the geomagnetic field. As shown in Fig 7.7, we obtain the inclination $\boldsymbol{C}_{g}^{f}$ of the fingertip with the gyroscope and accelerometer. Only the z-component of the geomagnetic field in the sensor frame can be compensated by the fused inclination $\boldsymbol{C}_{g}^{f}$. In this way, the z-component of the geomagnetic field disturbance is removed. The performance of this method depends on the location on earth since the z-component of the geomagnetic field varies at different locations. Except the geomagnetic disturbance, the magnetic surroundings may influence the performance of the proposed method. The estimation error depends on the magnetic surroundings' strength, direction etc.

In this experiment, only 8 healthy participants involved to do individual movements. Next step, we are going to apply the system to stroke patients and evaluate its performance under longer monitoring with sequential movements. As the strength of the magnet is much stronger than the geomagnetic field, it may cause unwanted forces if it is used near iron objects, such as iron tables or chairs. 


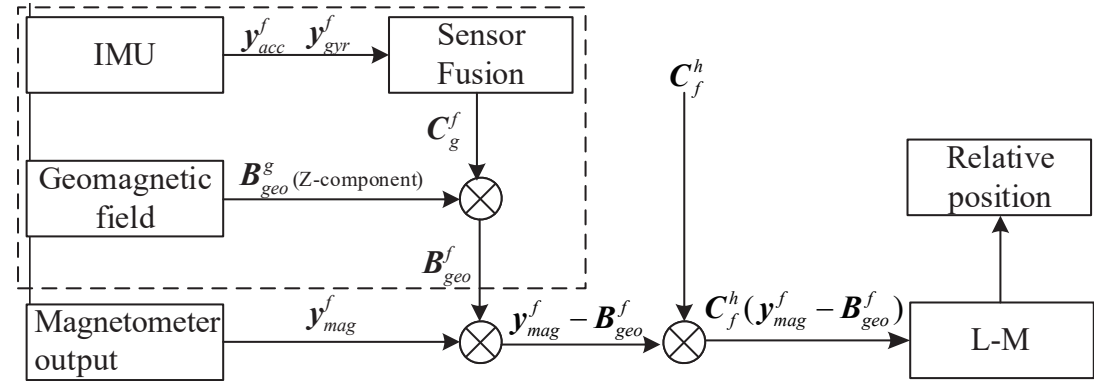

Figure 7.7: Illustration of the position accuracy improvement by compensating the z-axis component of geomagnetic field. $\boldsymbol{B}_{\text {geo }}^{g}$ is the $z$-axis component of geomagnetic field.

\subsection{Conclusion}

A small configuration based on IMMUs and a magnet was proposed to estimate fingertip poses of interest relative to the hand. The purpose of this approach is to avoid using prior information on the length of each segment and use as few IMMUs as possible. The fingertip orientations relative to the hand were firstly estimated by integrating the relative angular velocity. Subsequently, the fingertip position drift can be reduced by estimating fingertip orientations relative to the hand when the hand and fingers accelerate or rotate as a whole object, using the accelerometer and gyroscope. Then the relative positions were estimated using the relative orientations and magnetometer information. For rotating, grasping and writing movements, the estimated relative position and orientation errors between the hand and fingertips were $8.0 \sim 9.8 \mathrm{~mm}$ and $5.7^{\circ} \sim 11.2^{\circ}$ respectively. For the flexion and pinch movements, the relative position and orientation errors between the hand and fingertips were $16.2 \sim 25.2 \mathrm{~mm}$ and $20.6^{\circ} \sim 21.7^{\circ}$ respectively. In conclusion, the proposed method is a promising approach for the handfinger motion tracking with comparable performance as previous methods that applied the kinematic model of the fingers and additional IMMUs on intermediate finger segments. 
CHAPTER 8

\section{General discussion and conclusion}




\subsection{Introduction}

Accurate and minimally obtrusive 3D tracking of hand and finger pose during daily-life has many applications, including human-computer interface, virtual reality and rehabilitation, but has not been adequately solved to-date. This thesis presents and evaluates new sensing and analysis concepts that combine inertial and magnetic sensing. The objective of the research reported in this $\mathrm{PhD}$ thesis is to estimate the pose of thumb and index finger relative to the dorsal side of the hand using a sparse sensing set that has the potential becoming low obtrusive in daily-life use.

This research was divided in two parts, including calibration of the 3D magnetometers (part 1) and estimation of the pose of the fingertip relative to the dorsal side of the hand using a sparse configuration of inertial and magnetic measurement units on the dorsal side of the hand and involved fingertips, and a permanent magnet on the dorsal side of the hand (part 2).

In this final chapter, we will discuss the progress that the research reported in this $\mathrm{PhD}$ research has made relative to the state of the art, indicate limitations and future perspective (section 8.2), and draw conclusion relative to the objective set in the Introduction of this thesis (section 8.3).

\subsection{Discussion and future perspectives}

In this thesis, we proposed a method to estimate the poses of fingertips relative to the hand with a minimum IMMU-based setup, and good performance was achieved. After reducing the intermediate IMMUs of traditional IMMUbased tracking system [25, 26], we lose the information of intermediate segments and kinematic chain rule is not valid anymore. The fingertip position can not be estimated with the traditional methods $[25,26]$, which needs the length and orientation of each segment. The introduction of a passive magnetic localization system proved to be very successful and we only need to add a magnet on the dorsal side of the hand.

Although we proposed a minimum sensor setup and corresponding algorithms to estimate the pose of fingertips, several issues still need to be discussed, such as the number of IMMUs and finger tracking with only magnetometers. 


\section{Number of IMMUs}

We proposed a system with three IMMUs, which are attached on the tip of the thumb, index finger and dorsal side of the hand. Compared with traditional IMMU-based hand motion tracking systems [25, 26], we reduced the number of IMMUs as much as possible and only retained the IMMU on the tip of thumb and index finger, since they are most relavant to characterize the functional task performed by the hand. By reducing the number of IMMUs, the potential practicability of the system can be greatly improved. However, the cost of reducing the number of IMMUs is that we lose the information of intermediate segment and the principles of the kinematic chain rule can no longer be applied. However, we solved the problem by introducing a passive magnetic tracking system to estimate the position of the fingertips. In this case a kinematic chain is not needed. As far as we know, this is the first attempt to estimate the fingertip pose with a minimum IMMU setup. Some researchers attempt to track the hand movement with only one IMMU. However, it can only estimate the orientation of one segment, which is often applied to daily activity recognition [188]. Such approach can help collect some information about the user and the environment, but not accurate pose of fingers.

Existing methods to estimate the position of ferromagnetic object make need at least two magnetometers [187, 189], and enough distances are required between magnetometers in order to get different measurements. It is of great significance for localization in a small area that can only contain one magnetometer with our approach. However, the cost of using one magnetometers is that the orientation information must be known, and the accuracy of orientation estimation directly influences the position accuracy. An interesting alternative to investigate further would be to add one magnetometer on the fingertip while keeping the size of measurement unit the same. The differential magnetic field can help to get a better position performance.

\section{Finger tracking with only magnetometers}

In order to estimate the position of fingertips, we proposed to merge the passive magnetic localization information with inertial sensor information. Our idea was inspired by the previous research that exploited a magnetometer only to estimate the position of fingertips. 'AuraRing' [15] is a system that 
contains an AC magnetic transmitter on the distal segment of the index finger and three receivers on the wrist. The fingertip position can be estimated with a magnetic dipole model. The 'Manumer' has a similar configuration as the 'AuraRing' with a transmitter on the index finger and multi receivers on the wrist [190]. There are two major differences between 'AuraRing' and 'Manumer'. Firstly, 'Manumer' exploits a magnet as a transmitter while the 'AuraRing' uses an AC magnetic transmitter. Secondly, 'Manumer' exploits a magnetic localization system as a 'gray box', which needs a batch of data to train the relation between the position and magnetometer outputs. While 'AuraRing' exploits magnetic dipole model to obtain the position of distal segment of index finger. 'uTrack' [191] has a similar configuration as the 'Manumer', while its receiver on the ring finger instead of the wrist. Furthermore, it exploited the dipole model instead of machine learning method.

Compared with 'AuraRing', 'Manumer' and 'uTrack' system, our system firstly exploits inertial sensor to obtain the orientation information. Subsequently, the orientation information is fused with a magnetometer to obtain the position of fingertips relative to the hand. Furthermore, our system tracked two fingertips (more fingertips can be tracked when more IMMUs are on other fingertips). For the 'Manumer' and 'uTrack' system, it would be quite difficult to track multiple fingertips. Since when magnets are close to each other, the magnetic fields generated by these magnets cannot be separated easily.

\section{Passive magnetic tracking or active magnetic tracking}

Magnetic tracking is a good approach for estimating position of a fingertip. There are two basic methods: passive magnetic tracking and active magnetic tracking, but which method to prefer is yet undecided. Active magnetic tracking exploits an AC magnetic source as transmitter. The benefits are that it can filter the geomagnetic field and it has advantages for tracking multiple targets, since different targets can be easily separated by different frequencies. For example, the system 'Finexus' can track the pose of fingers relative to the hand, including thumb, index finger and middle finger [16]. However, it needs three AC magnetic source and external attachments such as power amplifiers. Moreover, they simplify the finger movement into 2DoF movement for an easier calculation, which is reasonable except for the thumb. The disadvantages make the active magnetic tracking difficult to use in ambulatory situations. On the other side, the passive magnetic 
tracking method needs a permanent magnet as a transmitter, the advantages are that it has small volume and does not consume any power. However, it faces the problem that the geomagnetic field is hard to be separated from the target magnetic field. Moreover, it has difficulties to track multiple targets when multiple magnets are attached on different fingertips. In this thesis, we pay more attention to small size of the system and ambulatory measurement. Thus, we chose passive magnetic localization system. To overcome the drawbacks of a passive localization system, we did two efforts. Firstly, we choose a strongest magnet with allowable size. Thus, the influence of geomagnetic disturbance can be minimized. Secondly, we optimized the configuration for multiple targets localization with passive localization system. Compared with 'Manumer' and 'uTrack' system [191, 190] , our system has one magnet on the dorsal side of hand and one receiver on each fingertip, rather than the magnet on the fingertip. The benefit of our configuration is that we do not need to distinguish between sources, while different fingertips can be easily identified from their own receivers.

\section{Biomechanical constraints or not}

Controlling orientation and position drift is one of the biggest challenges in the IMMU-based motion tracking. Some researchers proposed to use biomechanical constraints to compensate for orientation drift $[26,179]$. They assume most joints (except for the thumb) can only perform 2DoF rotation, instead of 3DoF rotation. The biomechanical constraints may indeed compensate the orientation drifts. However, it may also introduce unexpected orientation errors. Moreover, the IMMU needs to be well calibrated to its corresponding segment. The non-ideal calibration may introduce errors when we apply the constraints. Our method is a more general method for the estimation of finger pose and can be applied to different types of hand anatomies, since we did not use biomechanical constraints. Our method is more suitable for flexible fingers such as thumb and for the case when the hand and fingers are deformed due to osteoarthritis.

Still, our method is not a perfect solution to estimate the pose of fingertips, the error of orientation estimation based on the proposed method depends on the quality of the hand movement. The orientation drifts may not be well compensated if there is no designated-event where the hand moves as a whole object. Thus, the next step we may consider is to combine biomechanical constraints. When there is no designated-event available, we could com- 
bine biomechanical constraints to compensate the orientation drifts while giving up biomechanical constraints when the designated-event is available.

\section{Calibration of magnetometers}

Calibration of magnetometers is an essential step before use. Existing calibration methods were demonstrated effective in many fields, such as electronic compass [192]. However, this is not always true. Our method solve the calibration problem when the magnetometer is fixed on a huge object and can not be rotated in 3D space, while traditional methods fail. However, there are some common drawbacks in existing and our algorithms. The magnetic disturbances caused by the carrier of the magnetometer were simplified as soft-iron and hard-iron disturbance, where error parameters were linear [193, 194]. However, in many scenarios, the disturbances caused by the carrier are complicated, such as when the magnetometer is installed on a car or an aircraft etc. In such situations, the disturbances are nonlinear and often contain other error sources, such as eddy current magnetic disturbance $[195,196]$. Thus, the error model of the calibration needs to be improved.

The geomagnetic field was used as a reference in Chapter 2 and 3. However, many applications occur in indoor environments, where the geomagnetic field is easily disturbed by objects, such as iron beams used for construction, or tables and chairs [197, 198]. In addition, the magnetic inclination is also often disturbed indoors. This influences the alignment between magnetometer and accelerometer. Thus, it is of great significance to propose methods suitable for calibration in indoor environments. For example, the magnetic field generated by a coil can be used to replace the geomagnetic field as a reference. The differential coil magnetic field can reduce most of indoor disturbances. A coil that can generate uniform magnetic field could be a good choice, such as a Helmhertz coil. However, such coils are often expensive. A solenoid coil is a good compromise between uniformity and price. If the coil is placed horizontally, the coil magnetic field can be perpendicular to gravity. The angle between the magnetic field and gravity can be used to calibrate the misalignment between the magnetometer and accelerometer, instead of the magnetic inclination. 


\subsection{Conclusion}

In conclusion, we have proposed and evaluated novel methods to estimate the pose of fingertips relative to the dorsal side of the hand with a sparse set of inertial and magnetic measurement units and a permanent magnet.

In order to enable accurate magnetic sensing, we first proposed more efficient calibration methods for magnetometer arrays and for the case that the magnetometer is restricted in space. We have demonstrated adequate performance of these calibration methods.

The novel sensing methods for estimating the relative orientation of fingertips and the dorsal side of the hand only required inertial measurement units on involved finger tips and the dorsal side of the hand, and made use of the assumption that the hand and fingertips experience approximately the same angular velocity and acceleration if they move together, which regularly occurs in many daily-life tasks. We demonstrated that this can indeed result in regular updates of 3D relative orientation estimation, which can be combined with estimation of orientation changes in between using integration of measured angular velocities of the dorsal side of the hand and involved finger tips.

This thesis also demonstrated that positions of the finger tips relative to the dorsal side of the hand can be estimated using a permanent magnet on the dorsal side of the hand and magnetometers on the finger tips if their relative $3 \mathrm{D}$ orientation is known.

Finally, this thesis demonstrated that relative orientation and positions of finger tips to the dorsal side of the hand can be estimated when combining these methods.

Until now, the research problems proposed in the introduction are solved.

\section{Solutions to the research problems}

Research problem 1: How can we estimate the orientation of the fingertip when the geomagnetic field is heavily disturbed?

Solution: In many practical situations, the magnetic field is heavily disturbed. Therefore, drift in the yaw angle cannot be compensated using the magnetic field. We proposed to use the information that when hand and fingers move as a whole object, they share approximately the same angular 
velocity and acceleration. The performance of this method depends on the quality of the movement, when whole hand moves as an object, the quality of the movement is assumed as high since we have the opportunity to compensate the drift, the orientation error is smaller than 10 degrees in a functional water-drinking task. Otherwise, the movement quality is assumed to be low and the drift will continue. Compared with the traditional methods that exploit magnetometer and geomagnetic field to compensate the yaw drift [101], our method only uses the inertial sensor to estimate fingertip orientation relative to the hand, which is fundamentally immune to magnetic interference and has high potential to work under a highly complex indoor environment.

Research problem 2: Can we accurately and reliably estimate the fingertip position with one magnetometer and magnet?

Solution: To estimate the position of the fingertip, a magnet is attached on the back of hand. Existing methods assume that the magnet can be modeled as a dipole and multiple (more than two) magnetometers are needed for the localization $[169,187]$. We proposed a minimum measurement setup that only contains one IMMU on the fingertip and a new algorithm for the estimation of fingertip. In this approach, the orientation estimated by the inertial sensor is exploited. The experimental results show that the position error was less than $9 \%$ during flexion, abduction and pinch movements.

It should be noted that the accuracy of estimated relative orientation and position vary depending on conditions, the designated-event influence the estimation results. We expect that the sparse sensing method that we have proposed and evaluated has potential for use under daily-life conditions even in the case of deformed hands and fingers. The usefulness of our methods is however still to be demonstrated in practical applications. 


\section{Bibliography}

[1] James M Rehg and Takeo Kanade. Digiteyes: Vision-based hand tracking for human-computer interaction. In Proceedings of 1994 IEEE Workshop on Motion of Non-rigid and Articulated Objects, pages 16-22. IEEE, 1994.

[2] Cristina Manresa, Javier Varona, Ramon Mas, and Francisco J Perales. Hand tracking and gesture recognition for human-computer interaction. ELCVIA: electronic letters on computer vision and image analysis, pages 96-104, 2005.

[3] Cao Nguyen Khoa Nam, Hee Jun Kang, and Young Soo Suh. Golf swing motion tracking using inertial sensors and a stereo camera. IEEE Transactions on Instrumentation and measurement, 63(4):943952, 2013.

[4] Matej Perše, Matej Kristan, Stanislav Kovačič, Goran Vučkovič, and Janez Perš. A trajectory-based analysis of coordinated team activity in a basketball game. Computer Vision and Image Understanding, 113(5):612-621, 2009.

[5] Zhelong Wang, Jiaxin Wang, Hongyu Zhao, Sen Qiu, Jie Li, Fengshan Gao, and Xin Shi. Using wearable sensors to capture posture of the human lumbar spine in competitive swimming. IEEE Transactions on Human-Machine Systems, 49(2):194-205, 2019.

[6] Charles R Cameron, Louis W DiValentin, Rohini Manaktala, Adam C McElhaney, Christopher H Nostrand, Owen J Quinlan, Lauren N Sharpe, Adam C Slagle, Charles D Wood, Yang Yang Zheng, et al. Hand tracking and visualization in a virtual reality simulation. In 2011 IEEE systems and information engineering design symposium, pages 127-132. IEEE, 2011.

[7] Stefano Scheggi, Leonardo Meli, Claudio Pacchierotti, and Domenico Prattichizzo. Touch the virtual reality: using the leap motion controller for hand tracking and wearable tactile devices for immersive haptic rendering. In ACM SIGGRAPH 2015 Posters, pages 1-1. 2015. 
[8] Shayan Bahadori, Tikki Immins, and Thomas W Wainwright. A review of wearable motion tracking systems used in rehabilitation following hip and knee replacement. Journal of rehabilitation and assistive technologies engineering, 5:2055668318771816, 2018.

[9] Pasquale Daponte, Luca De Vito, Maria Riccio, and Carmine Sementa. Design and validation of a motion-tracking system for rom measurements in home rehabilitation. Measurement, 55:82-96, 2014.

[10] Huiyu Zhou and Huosheng Hu. Human motion tracking for rehabilitation-a survey. Biomedical signal processing and control, 3(1):1-18, 2008.

[11] Rasool Khadem, Clement C Yeh, Mohammad Sadeghi-Tehrani, Michael R Bax, Jeremy A Johnson, Jacqueline Nerney Welch, Eric P Wilkinson, and Ramin Shahidi. Comparative tracking error analysis of five different optical tracking systems. Computer Aided Surgery, 5(2):98-107, 2000.

[12] Josien C van den Noort, Henk G Kortier, Nathalie van Beek, DirkJan HEJ Veeger, and Peter H Veltink. Measuring 3d hand and finger kinematics - a comparison between inertial sensing and an optoelectronic marker system. PLoS One, 11(11):e0164889, 2016.

[13] Zhenbao Liu, Jinxin Huang, Junwei Han, Shuhui Bu, and Jianfeng Lv. Human motion tracking by multiple rgbd cameras. IEEE Transactions on Circuits and Systems for Video Technology, 27(9):20142027, 2016.

[14] Licong Zhang, Jürgen Sturm, Daniel Cremers, and Dongheui Lee. Real-time human motion tracking using multiple depth cameras. In 2012 IEEE/RSJ International Conference on Intelligent Robots and Systems, pages 2389-2395. IEEE, 2012.

[15] Farshid Salemi Parizi, Eric Whitmire, and Shwetak Patel. Auraring: Precise electromagnetic finger tracking. Proceedings of the ACM on Interactive, Mobile, Wearable and Ubiquitous Technologies, 3(4):128, 2019.

[16] Ke-Yu Chen, Shwetak N Patel, and Sean Keller. Finexus: Tracking precise motions of multiple fingertips using magnetic sensing. In 
Proceedings of the 2016 CHI Conference on Human Factors in Computing Systems, pages 1504-1514, 2016.

[17] Xiaochi Gu, Yifei Zhang, Weize Sun, Yuanzhe Bian, Dao Zhou, and Per Ola Kristensson. Dexmo: An inexpensive and lightweight mechanical exoskeleton for motion capture and force feedback in vr. In Proceedings of the 2016 CHI Conference on Human Factors in Computing Systems, pages 1991-1995, 2016.

[18] David J Sturman and David Zeltzer. A survey of glove-based input. IEEE Computer graphics and Applications, 14(1):30-39, 1994.

[19] Nattapong Tongrod, Teerakiat Kerdcharoen, Natthapol Watthanawisuth, and Adisorn Tuantranont. A low-cost data-glove for human computer interaction based on ink-jet printed sensors and zigbee networks. In International Symposium on Wearable Computers (ISWC) 2010, pages 1-2. IEEE, 2010.

[20] Bin Tian, Weijing Yao, Pan Zeng, Xuan Li, Huanjun Wang, Li Liu, Yu Feng, Chengsheng Luo, and Wei Wu. All-printed, low-cost, tunable sensing range strain sensors based on ag nanodendrite conductive inks for wearable electronics. Journal of Materials Chemistry $C$, 7(4):809-818, 2019.

[21] Eric Fujiwara, Murilo Ferreira Marques dos Santos, and Carlos K Suzuki. Flexible optical fiber bending transducer for application in glove-based sensors. IEEE Sensors Journal, 14(10):3631-3636, 2014.

[22] Alexandre Ferreira Da Silva, Anselmo Filipe Gonçalves, Paulo Mateus Mendes, and José Higino Correia. Fbg sensing glove for monitoring hand posture. IEEE Sensors Journal, 11(10):2442-2448, 2011.

[23] Federico Lorussi, Enzo Pasquale Scilingo, Mario Tesconi, Alessandro Tognetti, and Danilo De Rossi. Strain sensing fabric for hand posture and gesture monitoring. IEEE transactions on information technology in biomedicine, 9(3):372-381, 2005.

[24] Federica Ferrara, Riccardo Izzo, Andrea Ceciliani, and Alfredo Pio Di Tore. Pilot study on the testing of power glove applied to volleyball. 2019. 
[25] Henk G Kortier, Victor I Sluiter, Daniel Roetenberg, and Peter H Veltink. Assessment of hand kinematics using inertial and magnetic sensors. Journal of neuroengineering and rehabilitation, 11(1):1-15, 2014.

[26] Christina Salchow-Hömmen, Leonie Callies, Daniel Laidig, Markus Valtin, Thomas Schauer, and Thomas Seel. A Tangible Solution for Hand Motion Tracking in Clinical Applications. Sensors (Basel, Switzerland), 19(1), 2019.

[27] Changyu He, Peter Kazanzides, Hasan Tutkun Sen, Sungmin Kim, and Yue Liu. An inertial and optical sensor fusion approach for six degree-of-freedom pose estimation. Sensors, 15(7):16448-16465, 2015.

[28] Hongliang Ren and Peter Kazanzides. Investigation of attitude tracking using an integrated inertial and magnetic navigation system for hand-held surgical instruments. IEEE/ASME Transactions on Mechatronics, 17(2):210-217, 2010.

[29] A. Schwarz, Mmc Bhagubai, G. Wolterink, Jpo Held, and P. H. Veltink. Assessment of upper limb movement impairments after stroke using wearable inertial sensing. Sensors (Basel, Switzerland), 20(17):4770, 2020.

[30] Vladimir Kubelka and Michal Reinstein. Complementary filtering approach to orientation estimation using inertial sensors only. In 2012 IEEE international conference on robotics and automation, pages 599-605. IEEE, 2012.

[31] Anthony Kim and MF Golnaraghi. A quaternion-based orientation estimation algorithm using an inertial measurement unit. In PLANS 2004. Position Location and Navigation Symposium (IEEE Cat. No. 04CH37556), pages 268-272. IEEE, 2004.

[32] Sebastian $\mathrm{OH}$ Madgwick, Andrew JL Harrison, and Ravi Vaidyanathan. Estimation of imu and marg orientation using a gradient descent algorithm. In 2011 IEEE international conference on rehabilitation robotics, pages 1-7. IEEE, 2011. 
[33] Gabriele Ligorio and Angelo M Sabatini. A linear kalman filteringbased approach for $3 \mathrm{~d}$ orientation estimation from magnetic/inertial sensors. In 2015 IEEE International Conference on Multisensor Fusion and Integration for Intelligent Systems (MFI), pages 77-82. IEEE, 2015.

[34] Tatsuya Harada, Taketoshi Mori, and Tomomasa Sato. Development of a tiny orientation estimation device to operate under motion and magnetic disturbance. The International Journal of Robotics Research, 26(6):547-559, 2007.

[35] Bingfei Fan, Qingguo Li, and Tao Liu. How magnetic disturbance influences the attitude and heading in magnetic and inertial sensorbased orientation estimation. Sensors, 18(1):76, 2018.

[36] Shuozhi Yang and Qingguo Li. Inertial sensor-based methods in walking speed estimation: A systematic review. Sensors, 12(5):61026116, 2012.

[37] Tran Nhat Hung and Young Soo Suh. Inertial sensor-based two feet motion tracking for gait analysis. Sensors, 13(5):5614-5629, 2013.

[38] Seungwoo Lee, Byounggeun Kim, Hoon Kim, Rhan Ha, and Hojung Cha. Inertial sensor-based indoor pedestrian localization with minimum 802.15. 4a configuration. IEEE Transactions on Industrial Informatics, 7(3):455-466, 2011.

[39] Yongde Zhang, Kairui Wang, Jingang Jiang, and Qiyun Tan. Research on intraoperative organ motion tracking method based on fusion of inertial and electromagnetic navigation. IEEE Access, 9:49069-49081, 2021.

[40] Daniel Roetenberg, Chris TM Baten, and Peter H Veltink. Estimating body segment orientation by applying inertial and magnetic sensing near ferromagnetic materials. IEEE transactions on neural systems and rehabilitation engineering, 15(3):469-471, 2007.

[41] H Martin Schepers and Petrus H Veltink. Stochastic magnetic measurement model for relative position and orientation estimation. Measurement science and technology, 21(6):1-10, 2010. 
[42] Henk G Kortier, Jacob Antonsson, H Martin Schepers, Fredrik Gustafsson, and Peter H Veltink. Hand pose estimation by fusion of inertial and magnetic sensing aided by a permanent magnet. IEEE transactions on neural systems and rehabilitation engineering, 23(5):796-806, 2014.

[43] Arie Sheinker, Boaz Lerner, Nizan Salomonski, Boris Ginzburg, Lev Frumkis, and Ben-Zion Kaplan. Localization and magnetic moment estimation of a ferromagnetic target by simulated annealing. Measurement Science and Technology, 18(11):3451, 2007.

[44] Chao Hu, Max Q-H Meng, and Mrinal Mandal. Efficient magnetic localization and orientation technique for capsule endoscopy. International Journal of Information Acquisition, 2(01):23-36, 2005.

[45] Shuang Song, Chao Hu, and Max Q-H Meng. Multiple objects positioning and identification method based on magnetic localization system. IEEE Transactions on Magnetics, 52(10):1-4, 2016.

[46] Merayo, JMG, Brauer, and Primdahl. Triaxial fluxgate gradiometer of high stability and linearity. Sensors and Actuators A Physical, 120(1):71-77, 2005.

[47] Pavel Ripka. Advances in fluxgate sensors. Sensors and Actuators A Physical, 106(1):8-14, 2003.

[48] N. Linford, P. Linford, L. Martin, and A. Payne. Recent results from the english heritage caesium magnetometer system in comparison with recent fluxgate gradiometers. Archaeological Prospection, 14(3):151-166, 2007.

[49] Ahmed Salem, Toshio Hamada, Asahina Joseph Kiyoshi, and Keisuke Ushijima. Detection of unexploded ordnance (uxo) using marine magnetic gradiometer data. Exploration Geophysics, 36(1):5-9, 2005.

[50] Andrew Sunderland, Alexey V. Veryaskin, Wayne Mcrae, Ju Li, and David G. Blair. Direct string magnetic gradiometer for space applications. 147(2):529-535, 2008. 
[51] H. F. Abdul Amir, Xiangchao Hu, Hongfeng Pang, Liangrui Fu, Dixiang Chen, Mengchun Pan, A. M. Korsunsky, and Z. Guo. Calibration and distortion field compensation of gradiometer and the improvement in object remote sensing. MATEC Web of Conferences, 59:01004, 2016.

[52] Hongfeng Pang, Mengchun Pan, Jinfei Chen, Ji Li, Qi Zhang, and Shitu Luo. Integrated calibration and magnetic disturbance compensation of three-axis magnetometers. Measurement, 93:409-413, 2016.

[53] zhiqiang zhang. Two-step calibration methods for miniature inertial and magnetic sensor units. IEEE Transactions on Industrial Electronics, 62(6):3714-3723, 2015.

[54] J. M. G Merayo, P Brauer, F Primdahl, J. R Petersen, and O. V Nielsen. Scalar calibration of vector magnetometers. Measurement Science and Technology, 11(2):120-132(13), 2000.

[55] C. C Foster and G. H Elkaim. Extension of a two-step calibration methodology to include nonorthogonal sensor axes. IEEE Transactions on Aerospace and Electronic Systems, 44(3):1070-1078, 2008.

[56] D. Gebre-Egziabher, G H Elkaim, J D Powell, and B W Parkinson. A non-linear, two-step estimation algorithm for calibrating solid-state strapdown magnetometers. In International St Petersburg Conference on Navigation Systems, pages 28-30.

[57] Demoz Gebreegziabher, Gabriel H. Elkaim, J. David Powell, and Bradford W. Parkinson. Calibration of strapdown magnetometers in magnetic field domain. Journal of Aerospace Engineering, 19(2):87102, 2006

[58] Hongfeng Pang, Ji Li, Dixiang Chen, Mengchun Pan, Shitu Luo, Qi Zhang, and Feilu Luo. Calibration of three-axis fluxgate magnetometers with nonlinear least square method. Measurement, 46(4):1600-1606, 2013.

[59] Timo Pylvänäinen. Automatic and adaptive calibration of $3 \mathrm{~d}$ field sensors. Applied Mathematical Modelling, 32(4):575-587, 2008. 
[60] S. Bonnet, C. Bassompierre, C. Godin, S. Lesecq, and A. Barraud. Calibration methods for inertial and magnetic sensors. Sensors and Actuators A: Physical, 156(2):302-311, 2009.

[61] Renaudin Valérie, Muhammad Haris Afzal, and Lachapelle Gérard. Complete triaxis magnetometer calibration in the magnetic domain. Journal of Sensors, 2010(1):23-59, 2010.

[62] Zhi Tian Wu, Xiao Ping Hu, and Mei Ping Wu. Constrained total least squares algorithm applied to three-axis magnetometer calibration. Applied Mechanics and Materials, 278-280:968-973, 2013.

[63] Zhitian $\mathrm{Wu}$, Xiaoping $\mathrm{Hu}$, Meiping $\mathrm{Wu}$, and Juliang Cao. Constrained total least-squares calibration of three-axis magnetometer for vehicular applications. Measurement Science and Technology, 24(9):095003, 2013.

[64] John L. Crassidis, Kok-Lam Lai, and Richard R. Harman. Real-time attitude-independent three-axis magnetometer calibration. Journal of Guidance, Control, and Dynamics, 28(1):115-120, 2005.

[65] S Turner, M. J Hall, S. A. C Harmon, and N Hillier. Calibration of a novel three-axis fluxgate gradiometer for space applications. IEEE Transactions on Magnetics, 51(1):1-4, 2015.

[66] R. E. Bracken, D. V. Smith, and P. J. Brown. Calibrating a tensor magnetic gradiometer using spin data. 2005.

[67] Dixiang Chen and Hongfeng Pang. Calibration of magnetic gradient tensor measurement array in magnetic anomaly detection. Proc Spie, 8759(1):47, 2013.

[68] Yu Huang and Li Hua Wu. Two-step complete calibration of magnetic vector gradiometer based on functional link artificial neural network and least squares. IEEE Sensors Journal, 16(11):4230-4237, 2016.

[69] Jan Včelák. Calibration of triaxial fluxgate gradiometer. Journal of Applied Physics, 99(8):968-973, 2006.

[70] Gang Yin, Yingtang Zhang, Hongbo Fan, Guang Zhang, and Guoquan Ren. Linear calibration method of magnetic gradient tensor system. Measurement, 56:8-18, 2014. 
[71] H U Auster, K H Fornacon, and E Georgescu. Calibration of fluxgate magnetometers using relativemotion. Measurement Science and Technology, 13(7):1124-1131, 2002.

[72] Junping Cai. In-field practical calibration of three-axis magnetometers. Proceedings of the International Technical Meeting of the Institute of Navigation, pages 67-73, 2010.

[73] Valérie Renaudin, Muhammad Haris Afzal, and Gérard Lachapelle. Complete triaxis magnetometer calibration in the magnetic domain. Journal of Sensors, 2010:1-10, 2010.

[74] N. Linford, P. Linford, L. Martin, and A. Payne. Recent results from the english heritage caesium magnetometer system in comparison with recent fluxgate gradiometers. Archaeological Prospection, 14(3):151-166, 2007.

[75] Douglas Macharet, Héctor Perezimaz, Paulo Rezeck, Guilherme Potje, Luiz Benyosef, André Wiermann, Gustavo Freitas, Luis Garcia, and Mario Campos. Autonomous aeromagnetic surveys using a fluxgate magnetometer. Sensors, 16(2169), 2016.

[76] Ahmed Salem, Toshio Hamada, Asahina Joseph Kiyoshi, and Keisuke Ushijima. Detection of unexploded ordnance (uxo) using marine magnetic gradiometer data. Exploration Geophysics, 36(1):5-9, 2005.

[77] J. M. G Merayo, P Brauer, F Primdahl, J. R Petersen, and O. V Nielsen. Scalar calibration of vector magnetometers. Measurement Science and Technology, 11(2):120-132(13), 2000.

[78] R. Alonso and M.D. Shuster. Twostep: A fast robust algorithm for attitude-independent magnetometer-bias determination. Journal of the Astronautical Sciences, 50(4):433-451, 2002.

[79] C. C Foster and G. H Elkaim. Extension of a two-step calibration methodology to include nonorthogonal sensor axes. IEEE Transactions on Aerospace and Electronic Systems, 44(3):1070-1078, 2008.

[80] H U Auster, K H Fornacon, and E Georgescu. Calibration of fluxgate magnetometers using relativemotion. Measurement Science and Technology, 13(7):1124-1131, 2002. 
[81] D. Gebre-Egziabher, G H Elkaim, J D Powell, and B W Parkinson. A non-linear, two-step estimation algorithm for calibrating solid-state strapdown magnetometers. In International St Petersburg Conference on Navigation Systems, pages 28-30.

[82] Demoz Gebreegziabher, Gabriel H. Elkaim, J. David Powell, and Bradford W. Parkinson. Calibration of strapdown magnetometers in magnetic field domain. Journal of Aerospace Engineering, 19(2):87102, 2006.

[83] Timo Pylvänäinen. Automatic and adaptive calibration of $3 \mathrm{~d}$ field sensors. Applied Mathematical Modelling, 32(4):575-587, 2008.

[84] Renaudin Valérie, Muhammad Haris Afzal, and Lachapelle Gérard. Complete triaxis magnetometer calibration in the magnetic domain. Journal of Sensors, 2010(1):23-59, 2010.

[85] Hongfeng Pang, Dixiang Chen, Mengchun Pan, and Shitu Luo. A new calibration method of three axis magnetometer with nonlinearity suppression. IEEE Transactions on Magnetics, 49(9):5011-5015, 2013.

[86] Hongfeng Pang, Ji Li, Dixiang Chen, Mengchun Pan, Shitu Luo, Qi Zhang, and Feilu Luo. Calibration of three-axis fluxgate magnetometers with nonlinear least square method. Measurement, 46(4):1600-1606, 2013.

[87] Hongfeng Pang, Mengchun Pan, Jinfei Chen, Ji Li, Qi Zhang, and Shitu Luo. Integrated calibration and magnetic disturbance compensation of three-axis magnetometers. Measurement, 93:409-413, 2016.

[88] Zhicheng Yang, Shenggang Yan, and Bin Li. Hybrid calibration method for three-axis gradiometer. IEEE Magnetics Letters, PP(99):1-1, 2017.

[89] A. Olivares, G. Ruiz-Garcia, G. Olivares, J. M. Gorriz, and J. Ramirez. Automatic determination of validity of input data used in ellipsoid fitting marg calibration algorithms. Sensors (Basel), 13(9):11797-817, 2013. 
[90] Zhitian $\mathrm{Wu}$, Xiaoping $\mathrm{Hu}$, Meiping Wu, and Juliang Cao. Attitudeindependent magnetometer calibration for marine magnetic surveys: regularization issue. Journal of Geophysics and Engineering, 10(4):045002, 2013.

[91] Valérie Renaudin, Muhammad Haris Afzal, and Gérard Lachapelle. Complete triaxis magnetometer calibration in the magnetic domain. Journal of Sensors, 2010:1-10, 2010.

[92] Joel N. Franklin. On tikhonov's method for ill-posed problems. Mathematics of Computation, 28(128):889-907, 1974.

[93] Hannes Höppner, Maximilian Große-Dunker, Georg Stillfried, Justin Bayer, and Patrick Van Der Smagt. Key insights into hand biomechanics: human grip stiffness can be decoupled from force by cocontraction and predicted from electromyography. Frontiers in neurorobotics, 11:17, 2017.

[94] Dan Cervone, Alexander D'Amour, Luke Bornn, and Kirk Goldsberry. Pointwise: Predicting points and valuing decisions in real time with nba optical tracking data. In Proceedings of the 8th MIT Sloan Sports Analytics Conference, Boston, MA, USA, volume 28, page 3, 2014.

[95] Bruce Carse, Barry Meadows, Roy Bowers, and Philip Rowe. Affordable clinical gait analysis: An assessment of the marker tracking accuracy of a new low-cost optical $3 \mathrm{~d}$ motion analysis system. Physiotherapy, 99(4):347-351, 2013.

[96] Angela Sorriento, Maria Bianca Porfido, Stefano Mazzoleni, Giuseppe Calvosa, Miria Tenucci, Gastone Ciuti, and Paolo Dario. Optical and electromagnetic tracking systems for biomedical applications: a critical review on potentialities and limitations. IEEE Reviews in Biomedical Engineering, 13:212-232, 2019.

[97] Guofang Xiao, Ester Bonmati, Stephen Thompson, Joe Evans, John Hipwell, Daniil Nikitichev, Kurinchi Gurusamy, Sébastien Ourselin, David J Hawkes, Brian Davidson, et al. Electromagnetic tracking in image-guided laparoscopic surgery: Comparison with optical tracking and feasibility study of a combined laparoscope and laparoscopic ultrasound system. Medical physics, 45(11):5094-5104, 2018. 
[98] Megumi Ota, Hiroshige Tateuchi, Takaya Hashiguchi, Takehiro Kato, Yasuhiro Ogino, Momoko Yamagata, and Noriaki Ichihashi. Verification of reliability and validity of motion analysis systems during bilateral squat using human pose tracking algorithm. Gait \& Posture, 2020.

[99] Jung Keun Lee, Edward J Park, and Stephen N Robinovitch. Estimation of attitude and external acceleration using inertial sensor measurement during various dynamic conditions. IEEE transactions on instrumentation and measurement, 61(8):2262-2273, 2012.

[100] Qilong Yuan, Ehsan Asadi, Qinghua Lu, Guilin Yang, and I-Ming Chen. Uncertainty-based imu orientation tracking algorithm for dynamic motions. IEEE Transactions on Mechatronics, 24(2):872-882, 2019.

[101] Sebastian OH Madgwick, Andrew JL Harrison, and Ravi Vaidyanathan. Estimation of imu and marg orientation using a gradient descent algorithm. In 2011 IEEE international conference on rehabilitation robotics, pages 1-7. IEEE, 2011.

[102] Henk G. Kortier, Victor I. Sluiter, Daniel Roetenberg, and Peter H. Veltink. Assessment of hand kinematics using inertial and magnetic sensors. Journal of NeuroEngineering and Rehabilitation, 11(1):7084, 2014.

[103] Daniel Roetenberg, Henk J Luinge, Chris TM Baten, and Peter $\mathrm{H}$ Veltink. Compensation of magnetic disturbances improves inertial and magnetic sensing of human body segment orientation. IEEE Transactions on neural systems and rehabilitation engineering, 13(3):395-405, 2005.

[104] Nagesh Yadav and Chris Bleakley. Accurate orientation estimation using ahrs under conditions of magnetic distortion. Sensors, 14(11):20008-20024, 2014.

[105] Thomas Seel, Thomas Schauer, and Jorg Raisch. Joint axis and position estimation from inertial measurement data by exploiting kinematic constraints. In IEEE International Conference on Control Applications (CCA), 2012, pages 45-49, Piscataway, NJ, 2012. IEEE. 
[106] Shaghayegh Zihajehzadeh and Edward J Park. A novel biomechanical model-aided imu/uwb fusion for magnetometer-free lower body motion capture. IEEE Transactions on Systems, Man, and Cybernetics: Systems, 47(6):927-938, 2016.

[107] Tobias Zimmermann, Bertram Taetz, and Gabriele Bleser. Imu-tosegment assignment and orientation alignment for the lower body using deep learning. Sensors, 18(1):302, 2018.

[108] Arash Atrsaei, Hassan Salarieh, and Aria Alasty. Human arm motion tracking by orientation-based fusion of inertial sensors and kinect using unscented kalman filter. Journal of biomechanical engineering, 138(9):1-13, 2016.

[109] Zhengyou Zhang. Microsoft kinect sensor and its effect. IEEE multimedia, 19(2):4-10, 2012.

[110] Erik E Stone and Marjorie Skubic. Fall detection in homes of older adults using the microsoft kinect. IEEE journal of biomedical and health informatics, 19(1):290-301, 2014.

[111] Ross A Clark, Yong-Hao Pua, Karine Fortin, Callan Ritchie, Kate E Webster, Linda Denehy, and Adam L Bryant. Validity of the microsoft kinect for assessment of postural control. Gait \& posture, 36(3):372$377,2012$.

[112] James Connolly, Joan Condell, Brendan O'Flynn, Javier Torres Sanchez, and Philip Gardiner. Imu sensor-based electronic goniometric glove for clinical finger movement analysis. IEEE Sensors Journal, 18(3):1273-1281, 2017.

[113] Adnan Rashid and Osman Hasan. Wearable technologies for hand joints monitoring for rehabilitation: A survey. Microelectronics Journal, 88:173-183, 2019.

[114] Florian Kral, Elisabeth J Puschban, Herbert Riechelmann, and Wolfgang Freysinger. Comparison of optical and electromagnetic tracking for navigated lateral skull base surgery. The International Journal of Medical Robotics and Computer Assisted Surgery, 9(2):247-252, 2013. 
[115] Daniel Roetenberg, Per J Slycke, and Peter H Veltink. Ambulatory position and orientation tracking fusing magnetic and inertial sensing. IEEE Transactions on Biomedical Engineering, 54(5):883-890, 2007.

[116] Brandon Wagstaff, Valentin Peretroukhin, and Jonathan Kelly. Robust data-driven zero-velocity detection for foot-mounted inertial navigation. IEEE Sensors Journal, 20(2):957-967, 2019.

[117] Michael B Del Rosario, Heba Khamis, Phillip Ngo, Nigel H Lovell, and Stephen J Redmond. Computationally efficient adaptive errorstate kalman filter for attitude estimation. IEEE Sensors Journal, 18(22):9332-9342, 2018.

[118] Joan Sola. Quaternion kinematics for the error-state kalman filter. arXiv preprint arXiv:1711.02508, pages 1-95, 2017.

[119] Dirk Weenk, Daniel Roetenberg, Bert-Jan JF van Beijnum, Hermie J Hermens, and Peter H Veltink. Ambulatory estimation of relative foot positions by fusing ultrasound and inertial sensor data. IEEE transactions on neural systems and rehabilitation engineering, 23(5):817826, 2014.

[120] Ming Hao, Ken Chen, and Chenglong Fu. Smoother-based 3-d foot trajectory estimation using inertial sensors. IEEE Transactions on Biomedical Engineering, 66(12):3534-3542, 2019.

[121] Ge Gao, Maria Kyrarini, Mohammad Razavi, Xingchen Wang, and Axel Gräser. Comparison of dynamic vision sensor-based and imubased systems for ankle joint angle gait analysis. In 20162 nd International Conference on Frontiers of Signal Processing (ICFSP), pages 93-98. IEEE, 2016.

[122] W-W Wang and L-C Fu. Mirror therapy with an exoskeleton upperlimb robot based on imu measurement system. In 2011 IEEE International Symposium on Medical Measurements and Applications, pages 370-375. IEEE, 2011.

[123] Simo Särkkä. Unscented rauch-tung-striebel smoother. IEEE transactions on automatic control, 53(3):845-849, 2008. 
[124] Simo Sarkka, Ville V Viikari, Miika Huusko, and Kaarle Jaakkola. Phase-based uhf rfid tracking with nonlinear kalman filtering and smoothing. IEEE Sensors Journal, 12(5):904-910, 2011.

[125] Woei-Leong Chan and Fei-Bin Hsiao. Implementation of the rauchtung-striebel smoother for sensor compatibility correction of a fixedwing unmanned air vehicle. Sensors, 11(4):3738-3764, 2011.

[126] Andrew D Wiles, David G Thompson, and Donald D Frantz. Accuracy assessment and interpretation for optical tracking systems. In Medical Imaging 2004: Visualization, Image-Guided Procedures, and Display, volume 5367, pages 421-432. International Society for Optics and Photonics, 2004.

[127] Bo Kyu Kwon, Soohee Han, Oh Kyu Kwon, and Wook Hyun Kwon. Minimum variance fir smoothers for discrete-time state space models. IEEE Signal Processing Letters, 14(8):557-560, 2007.

[128] Richard G Gibbs. Square root modified bryson-frazier smoother. IEEE transactions on automatic control, 56(2):452-456, 2010.

[129] K. Oka, Y. Sato, and H. Koike. Real-time fingertip tracking and gesture recognition. IEEE Computer Graphics and Applications, 22(6):64-71, 2002.

[130] Eugene Tunik, Soha Saleh, and Sergei V Adamovich. Visuomotor discordance during visually-guided hand movement in virtual reality modulates sensorimotor cortical activity in healthy and hemiparetic subjects. IEEE Transactions on Neural Systems and Rehabilitation Engineering, 21(2):198-207, 2013.

[131] Nicolas Vignais, Markus Miezal, Gabriele Bleser, Katharina Mura, Dominic Gorecky, and Frédéric Marin. Innovative system for realtime ergonomic feedback in industrial manufacturing. Applied ergonomics, 44(4):566-574, 2013.

[132] Tony Szturm, James F. Peters, Chris Otto, Naaz Kapadia, and Ankur Desai. Task-specific rehabilitation of finger-hand function using interactive computer gaming. Archives of physical medicine and rehabilitation, 89(11):2213-2217, 2008. 
[133] Norhafizan Ahmad, Raja Ariffin Raja Ghazilla, Nazirah M Khairi, and Vijayabaskar Kasi. Reviews on various inertial measurement unit (imu) sensor applications. International Journal of Signal Processing Systems, 1(2):256-262, 2013.

[134] R. H. Jebsen, N. Taylor, R. B. Trieschmann, M. J. Trotter, and L. A. Howard. An objective and standardized test of hand function. Archives of physical medicine and rehabilitation, 50(6):311319, 1969.

[135] Thomas Platz, Cosima Pinkowski, Frederike van Wijck, In-Ha Kim, Paolo Di Bella, and Garth Johnson. Reliability and validity of arm function assessment with standardized guidelines for the Fugl-Meyer Test, Action Research Arm Test and Box and Block Test: a multicentre study. Clinical rehabilitation, 19(4):404-411, 2005.

[136] A. Kapur, N. Virji-Babul, G. Tzanetakis, and P. F. Driessen. Gesturebased affective computing on motion capture data. volume 3784, pages $1-7$.

[137] Jože Guna, Grega Jakus, Matevž Pogačnik, Sašo Tomažič, and Jaka Sodnik. An analysis of the precision and reliability of the leap motion sensor and its suitability for static and dynamic tracking. Sensors (Basel, Switzerland), 14(2):3702-3720, 2014.

[138] Anna H Smeragliuolo, N Jeremy Hill, Luis Disla, and David Putrino. Validation of the leap motion controller using markered motion capture technology. Journal of biomechanics, 49(9):1742-1750, 2016.

[139] Wei Lu, Zheng Tong, and Jinghui Chu. Dynamic hand gesture recognition with leap motion controller. IEEE Signal Processing Letters, 23(9):1188-1192, 2016.

[140] Mohamed Mohandes, S Aliyu, and M Deriche. Arabic sign language recognition using the leap motion controller. In 2014 IEEE 23rd International Symposium on Industrial Electronics (ISIE), pages 960-965. IEEE, 2014.

[141] Michela Borghetti, Emilio Sardini, and Mauro Serpelloni. Sensorized Glove for Measuring Hand Finger Flexion for Rehabilitation Purposes. IEEE Transactions on Instrumentation and Measurement, 62(12):3308-3314, 2013. 
[142] Shuai Chen, Zheng Lou, Di Chen, Kai Jiang, and Guozhen Shen. Polymer-enhanced highly stretchable conductive fiber strain sensor used for electronic data gloves. Advanced Materials Technologies, 1(7):1600136, 2016.

[143] Ke-Yu Chen, Shwetak N. Patel, and Sean Keller. Finexus. In Jofish Kaye, Allison Druin, Cliff Lampe, Dan Morris, and Juan Pablo Hourcade, editors, CHI 2016, pages 1504-1514, New York, New York, 2016. The Association for Computing Machinery.

[144] Yinghong Ma, Zhi-Hong Mao, Wenyan Jia, Chengliu Li, Jiawei Yang, and Mingui Sun. Magnetic Hand Tracking for Human-Computer Interface. IEEE Transactions on Magnetics, 47(5):970-973, 2011.

[145] Bor-Shing Lin, Pei-Chi Hsiao, Shu-Yu Yang, Che-Shih Su, and I-Jung Lee. Data Glove System Embedded With Inertial Measurement Units for Hand Function Evaluation in Stroke Patients. IEEE transactions on neural systems and rehabilitation engineering : a publication of the IEEE Engineering in Medicine and Biology Society, 25(11):22042213, 2017.

[146] Hsien-Ting Chang and Jen-Yuan Chang. Sensor Glove based on Novel Inertial Sensor Fusion Control Algorithm for 3D Real-time Hand Gestures Measurements. IEEE Transactions on Industrial Electronics, page 1, 2019.

[147] Thomas Seel and Stefan Ruppin. Eliminating the Effect of Magnetic Disturbances on the Inclination Estimates of Inertial Sensors. IFACPapersOnLine, 50(1):8798-8803, 2017.

[148] Wolfgang Teufl, Markus Miezal, Bertram Taetz, Michael Fröhlich, and Gabriele Bleser. Validity, Test-Retest Reliability and Long-Term Stability of Magnetometer Free Inertial Sensor Based 3D Joint Kinematics. Sensors (Basel, Switzerland), 18(7), 2018.

[149] Daniel Laidig, Dustin Lehmann, Marc-André Bégin, and Thomas Seel. Magnetometer-free realtime inertial motion tracking by exploitation of kinematic constraints in 2-dof joints. In 201941 st Annual International Conference of the IEEE Engineering in Medicine and Biology Society (EMBC), pages 1233-1238. IEEE, 2019. 
[150] Dustin Lehmann, Daniel Laidig, and Thomas Seel. Magnetometerfree motion tracking of one-dimensional joints by exploiting kinematic constraints. Proceedings on Automation in Medical Engineering, 1(1):027-027, 2020.

[151] David Tedaldi, Alberto Pretto, and Emanuele Menegatti. A robust and easy to implement method for imu calibration without external equipments. In 2014 IEEE International Conference on Robotics and Automation (ICRA), pages 3042-3049. IEEE, 2014.

[152] WT Fong, SK Ong, and AYC Nee. Methods for in-field user calibration of an inertial measurement unit without external equipment. Measurement Science and technology, 19(8):085202, 2008.

[153] Stanley W Shepperd. Quaternion from rotation matrix. Journal of Guidance and Control, 1(3):223-224, 1978.

[154] Olivier Lambercy, Ludovic Dovat, Roger Gassert, Etienne Burdet, Chee Leong Teo, and Theodore Milner. A haptic knob for rehabilitation of hand function. IEEE Transactions on Neural Systems and Rehabilitation Engineering, 15(3):356-366, 2007.

[155] Gunes Yavuzer, Ruud Selles, Nebahat Sezer, Serap Sütbeyaz, Johannes B Bussmann, Füsun Köseoğlu, Mesut B Atay, and Henk J Stam. Mirror therapy improves hand function in subacute stroke: a randomized controlled trial. Archives of physical medicine and rehabilitation, 89(3):393-398, 2008.

[156] Rong Zhu and Zhaoying Zhou. A real-time articulated human motion tracking using tri-axis inertial/magnetic sensors package. IEEE Transactions on Neural systems and rehabilitation engineering, 12(2):295302, 2004.

[157] Gabriele Ligorio and Angelo M Sabatini. A novel kalman filter for human motion tracking with an inertial-based dynamic inclinometer. IEEE Transactions on Biomedical Engineering, 62(8):2033-2043, 2015.

[158] Jill Schmidt, Devin R Berg, Heidi-Lynn Ploeg, and Leone Ploeg. Precision, repeatability and accuracy of optotrak ${ }^{\circledR}$ optical motion tracking systems. International Journal of Experimental and Computational Biomechanics, 1(1):114-127, 2009. 
[159] Pierre Merriaux, Yohan Dupuis, Rémi Boutteau, Pascal Vasseur, and Xavier Savatier. A study of vicon system positioning performance. Sensors, 17(7):1591, 2017.

[160] Alexandra Pfister, Alexandre M West, Shaw Bronner, and Jack Adam Noah. Comparative abilities of microsoft kinect and vicon $3 \mathrm{~d}$ motion capture for gait analysis. Journal of medical engineering \& technology, 38(5):274-280, 2014.

[161] Zhicheng Yang, Bert-Jan F Van Beijnum, Bin Li, Shenggang Yan, and Peter H Veltink. Estimation of relative hand-finger orientation using a small imu configuration. Sensors, 20(14):4008, 2020.

[162] Daniel Laidig, Philipp Müller, and Thomas Seel. Automatic anatomical calibration for imu-based elbow angle measurement in disturbed magnetic fields. Current directions in biomedical engineering, 3(2):167-170, 2017.

[163] Azzurra Chiri, Nicola Vitiello, Francesco Giovacchini, Stefano Roccella, Fabrizio Vecchi, and Maria Chiara Carrozza. Mechatronic design and characterization of the index finger module of a hand exoskeleton for post-stroke rehabilitation. IEEE/ASmE Transactions on mechatronics, 17(5):884-894, 2011.

[164] Catherine E Lang, Stacey L DeJong, and Justin A Beebe. Recovery of thumb and finger extension and its relation to grasp performance after stroke. Journal of neurophysiology, 102(1):451-459, 2009.

[165] Chen Wang, Xiaojuan Zhang, Xiaodong Qu, Xiao Pan, Guangyou Fang, and Luzhao Chen. A modified magnetic gradient contraction based method for ferromagnetic target localization. Sensors, 16(12):2168, 2016.

[166] Zhicheng Yang, Shenggang Yan, and Bin Li. Hybrid calibration method for three-axis gradiometer. IEEE Magnetics Letters, 8:1-5, 2017.

[167] Manon Kok, Jeroen D Hol, Thomas B Schön, Fredrik Gustafsson, and Henk Luinge. Calibration of a magnetometer in combination with inertial sensors. In 2012 15th International Conference on Information Fusion, pages 787-793. IEEE, 2012. 
[168] Valérie Renaudin, Muhammad Haris Afzal, and Gérard Lachapelle. Complete triaxis magnetometer calibration in the magnetic domain. Journal of sensors, 2010, 2010.

[169] Roger Alimi, Nir Geron, Eyal Weiss, and Tsuriel Ram-Cohen. Ferromagnetic mass localization in check point configuration using a levenberg marquardt algorithm. Sensors, 9(11):8852-8862, 2009.

[170] Cheryl D Metcalf, Rebecca Robinson, Adam J Malpass, Tristan P Bogle, Thomas A Dell, Chris Harris, and Sara H Demain. Markerless motion capture and measurement of hand kinematics: validation and application to home-based upper limb rehabilitation. IEEE Transactions on Biomedical Engineering, 60(8):2184-2192, 2013.

[171] Na Jin Seo, Jayashree Arun Kumar, Pilwon Hur, Vincent Crocher, Binal Motawar, and Kishor Lakshminarayanan. Usability evaluation of low-cost virtual reality hand and arm rehabilitation games. Journal of rehabilitation research and development, 53(3):321, 2016.

[172] Jingchen Liu, Peter Carr, Robert T Collins, and Yanxi Liu. Tracking sports players with context-conditioned motion models. In Proceedings of the IEEE Conference on Computer Vision and Pattern Recognition, pages 1830-1837, 2013.

[173] Lorna Herda, Pascal Fua, Ralf Plänkers, Ronan Boulic, and Daniel Thalmann. Using skeleton-based tracking to increase the reliability of optical motion capture. Human movement science, 20(3):313-341, 2001.

[174] Johannes Hummel, Michael Figl, Wolfgang Birkfellner, Michael R Bax, R Shahidi, CR Maurer Jr, and H Bergmann. Evaluation of a new electromagnetic tracking system using a standardized assessment protocol. Physics in Medicine \& Biology, 51(10):N205, 2006.

[175] Peter M Mills, Steven Morrison, David G Lloyd, and Rod S Barrett. Repeatability of $3 \mathrm{~d}$ gait kinematics obtained from an electromagnetic tracking system during treadmill locomotion. Journal of biomechanics, 40(7):1504-1511, 2007.

[176] Xiaoping Yun, James Calusdian, Eric R Bachmann, and Robert B McGhee. Estimation of human foot motion during normal walking 
using inertial and magnetic sensor measurements. IEEE transactions on Instrumentation and Measurement, 61(7):2059-2072, 2012.

[177] Manon Kok and Thomas B Schön. A fast and robust algorithm for orientation estimation using inertial sensors. IEEE Signal Processing Letters, 26(11):1673-1677, 2019.

[178] Young Soo Suh. Orientation estimation using a quaternion-based indirect kalman filter with adaptive estimation of external acceleration. IEEE Transactions on Instrumentation and Measurement, 59(12):3296-3305, 2010.

[179] Thomas Seel, Thomas Schauer, and Jörg Raisch. Joint axis and position estimation from inertial measurement data by exploiting kinematic constraints. In 2012 IEEE International Conference on Control Applications, pages 45-49. IEEE, 2012.

[180] Jakub Galka, Mariusz Mkasior, Mateusz Zaborski, and Katarzyna Barczewska. Inertial motion sensing glove for sign language gesture acquisition and recognition. IEEE Sensors Journal, 16(16):63106316, 2016.

[181] Bor-Shing Lin, I Lee, Shu-Yu Yang, Yi-Chiang Lo, Junghsi Lee, JeanLon Chen, et al. Design of an inertial-sensor-based data glove for hand function evaluation. Sensors, 18(5):1545, 2018.

[182] Nima Enayati, Elena De Momi, and Giancarlo Ferrigno. A quaternion-based unscented kalman filter for robust optical/inertial motion tracking in computer-assisted surgery. IEEE Transactions on Instrumentation and Measurement, 64(8):2291-2301, 2015.

[183] Hongliang Ren, Denis Rank, Martin Merdes, Jan Stallkamp, and Peter Kazanzides. Multisensor data fusion in an integrated tracking system for endoscopic surgery. IEEE Transactions on Information Technology in Biomedicine, 16(1):106-111, 2011.

[184] Sen Qiu, Zhelong Wang, Hongyu Zhao, Kairong Qin, Zhenglin Li, and Huosheng Hu. Inertial/magnetic sensors based pedestrian dead reckoning by means of multi-sensor fusion. Information Fusion, 39:108-119, 2018. 
[185] H Martin Schepers, Daniel Roetenberg, and Peter H Veltink. Ambulatory human motion tracking by fusion of inertial and magnetic sensing with adaptive actuation. Medical \& biological engineering \& computing, 48(1):27, 2010.

[186] Zhicheng Yang, Shenggang Yan, Lianping Chen, and Bin Li. Ferromagnetic object localization based on improved triangulating and ranging. IEEE Magnetics Letters, 10:1-5, 2019.

[187] Hongfeng Pang, Shitu Luo, Qi Zhang, Ji Li, Dixiang Chen, Mengchun Pan, and Feilu Luo. Calibration of a fluxgate magnetometer array and its application in magnetic object localization. Measurement Science and Technology, 24(7):075102, 2013.

[188] Alessandra Moschetti, Laura Fiorini, Dario Esposito, Paolo Dario, and Filippo Cavallo. Daily activity recognition with inertial ring and bracelet: An unsupervised approach. In 2017 IEEE International Conference on Robotics and Automation (ICRA), pages 3250-3255. IEEE, 2017.

[189] Chen Wang, Xiaodong Qu, Xiaojuan Zhang, Wanhua Zhu, and Guangyou Fang. A fast calibration method for magnetometer array and the application of ferromagnetic target localization. IEEE Transactions on Instrumentation and Measurement, 66(7):17431750, 2017.

[190] Nizan Friedman, Justin B Rowe, David J Reinkensmeyer, and Mark Bachman. The manumeter: a wearable device for monitoring daily use of the wrist and fingers. IEEE journal of biomedical and health informatics, 18(6):1804-1812, 2014.

[191] Ke-Yu Chen, Kent Lyons, Sean White, and Shwetak Patel. utrack: 3d input using two magnetic sensors. In Proceedings of the 26th annual ACM symposium on User interface software and technology, pages 237-244, 2013.

[192] Jiancheng Fang, Hongwei Sun, Juanjuan Cao, Xiao Zhang, and Ye Tao. A novel calibration method of magnetic compass based on ellipsoid fitting. IEEE Transactions on Instrumentation and Measurement, 60(6):2053-2061, 2011. 
[193] Seyed Amir Hoseini Tabatabaei, Alexander Gluhak, and Rahim Tafazolli. A fast calibration method for triaxial magnetometers. IEEE Transactions on Instrumentation and Measurement, 62(11):29292937, 2013.

[194] Valérie Renaudin, Muhammad Haris Afzal, and Gérard Lachapelle. New method for magnetometers based orientation estimation. In IEEE/ION Position, Location and Navigation Symposium, pages 348356. IEEE, 2010.

[195] Samuel H Bickel. Small signal compensation of magnetic fields resulting from aircraft maneuvers. IEEE Transactions on aerospace and electronic systems, (4):518-525, 1979.

[196] L Tuck, Claire Samson, C Polowick, and Jeremy Laliberte. Real-time compensation of magnetic data acquired by a single-rotor unmanned aircraft system. Geophysical Prospecting, 67(6-Geophysical Instrumentation and Acquisition):1637-1651, 2019.

[197] Markus Hehn, Erik Sippel, Christian Carlowitz, and Martin Vossiek. High-accuracy localization and calibration for 5-dof indoor magnetic positioning systems. IEEE Transactions on Instrumentation and Measurement, 68(10):4135-4145, 2018.

[198] David Hanley, Alexander B Faustino, Scott D Zelman, David A Degenhardt, and Timothy Bretl. Magpie: A dataset for indoor positioning with magnetic anomalies. In 2017 International Conference on Indoor Positioning and Indoor Navigation (IPIN), pages 1-8. IEEE, 2017. 


\section{Acknowledgement}

Five years hard working, it finally pays off, my dissertation is ready! During the PhD journey, I had sorrows and pains, I had happiness and joy. Several times, I wanted to quit when I was in trouble with my $\mathrm{PhD}$ project. I often asked myself a question, why do the $\mathrm{PhD}$, because of the title? No! Earn more money in the future? No! Gradually, I realized that I really enjoy finding and solving problems, both theoretically and practically. Once our work was published, I was happy from the heart. I feel lucky that I started and finished the $\mathrm{PhD}$ project.

I cannot image to finish this without the help of a number of people. First of all, I want to thank my supervisor Peter Veltink and co-supervisor BertJan, who supervised me through four chapters of my thesis at UT. Speaking of Peter, he is a very responsible mentor that he spent lots of time and energy on my $\mathrm{PhD}$, from discussing the original ideal to writing the thesis. His encouragement is one of the important reasons that I did not give up when I had a difficult time. When I was doing my PhD in UT, I felt at ease because I knew Peter was always there and ready for help. Also, his optimism and hardworking influence me a lot. Bert-Jan is a very friendly co-supervisor. Also, he is a very good listener and advisor. He guided me with the conception and structure of the thesis. We discussed a lot about the content, the paper writing and response to the reviewers. We had a great time together.

Fortunately, I also have two supervisors at NPU, Shenggang Yan and Bin Li, who guided me through two chapters of my thesis. They created good conditions for my study and work at NPU. Shenggang Yan is a persuasive supervisor and he gave me lots of useful suggestions for my $\mathrm{PhD}$ program. I still remember the scene where we discussed the draft of our first journal paper, that publication gave me a lot of confidence. Bin Li and I discussed a lot about the magnetometer, such as calibration and localization. He provided me equipment for the experiment. Apart from that, he is also a very good friend, took care of me when I in trouble out of work.

Next to my supervisors, I would like to thank my colleagues and friends. I had a very pleasant time in BSS group. Sandra, Sylvia Ed and Marcel provided me strong support. I had three excellent colleagues sharing the same office, Irfan, Boudewijn and Niels, it was very pleasant working with them, thanks for your accompany and help. I would not list all the people 
in BSS, like Kostas, Carlijn, Fahim, Roelof. But it was very enjoyable to work with you guys. Also the coffee break and drinking night. Except BSS, I would like to thank L. Schaake from RRD, he provided me lots of help for using the optical tracking system and data processing. I also want to thank my friends Zhiwei Guo, Kaifang Liu, Qiansong Zhang, Xihe Li, Shuijing Jie, Xiangyi meng etc. We had great time together during the weekends, for the cooking, walking, cycling, and playing poker.

Finally, I would like to thank my families and girl friend Fang Wang, for their continuous support and encouragement! 


\section{About the author}

Zhicheng Yang was born in Xuchang, China, in 1991. He finished his high school locally in 2009. Subsequently, he started studying electrical engineering at Zhengzhou University, Zhengzhou, China. In September 2013, he joined Prof Changmao Yang's team at Yichang Testing Technology Research Institute, Hubei, China as a master student, where he was responsible for designing a Giant-Magneto-Impedance-based magnetic sensor.

In September 2016, he started his PHD at Prof Shenggang Yan and Prof Bin Li's team, where he was concentrating on developing application algorithms for magnetometers. Since October 2018, he joined Biomedical Signals and Signals group as a double PHD student under supervision of prof. dr. ir. P. H. Veltink and dr.ir. B.J.F. van Beijnum, where he was concentrating

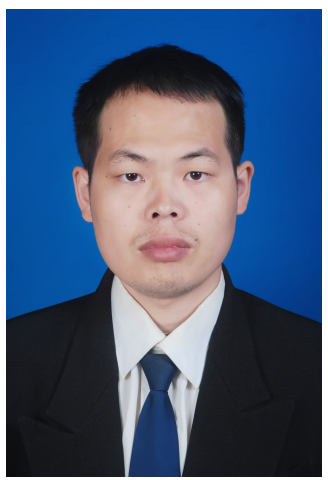
on the hand finger movement tracking with inertial sensors and magnetometers. His research interests include magnetic/inertial sensor calibration, detection and localization of ferromagnetic objects and motion tracking with inertial sensors etc. 


\section{List of publications}

\section{Journal papers}

1. Yang, Z., Yan, S., \& Li, B. (2017). Hybrid calibration method for three-axis gradiometer. IEEE Magnetics Letters, 8, 1-5.

2. Yang, Z., Bin, L., \& Lianping, C. (2018). Calibration of tri-axis magnetometers using an improved truncated singular value decomposition method. Measurement Science and Technology, 29(12), 125101.

3. Yang, Z., Yan, S., van Beijnum, B. J. F., Li, B., \& Veltink, P. H. (2021). Improvement of Optical Tracking-Based Orientation Estimation by Fusing Gyroscope Information. IEEE Transactions on Instrumentation and Measurement, 70, 1-13.

4. Yang, Z., Van Beijnum, B. J. F., Li, B., Yan, S., \& Veltink, P. H. (2020). Estimation of Relative Hand-Finger Orientation Using a Small IMU Configuration. Sensors, 20(14), 4008.

5. Yang, Z., Yan, S., Van Beijnum, B. J. F., Li, B., \& Veltink, P. H. (2021). Estimate Hand-Finger Position With One Magnetometer and Known Relative Orientation. IEEE Transactions on Instrumentation and Measurement, 70, 1-11.

6. Yang, Z., Yan, S., van Beijnum, B. J. F., Li, B., \& Veltink, P. H. (2021). Hand-finger pose estimation using inertial sensors, magnetic sensors and a magnet. IEEE Sensors Journal. 
